\title{
Análisis numérico de fenómenos de fisuración por contracción en estructuras de hormigón simple
}

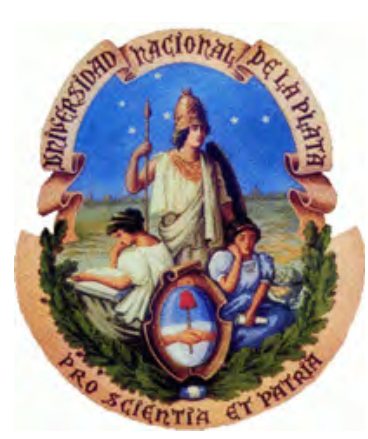

Tesis doctoral presentada por

Ing. María Paula Zappitelli

ante la

Facultad de Ingeniería de la

Universidad Nacional de La Plata

para acceder al grado de

DOCTORA EN INGENIERÍA

Dirección de tesis:

Director: Dr. Ing. Claudio Guillermo Rocco,

Codirector: Dr. Ing. Edgardo Ignacio Villa

La Plata,15 de abril de 2021 
Documento maquetado con TEXIS v.1.0+.

Este documento está preparado para ser imprimido a doble cara. 
Análisis numérico de fenómenos de fisuración por contracción en estructuras de hormigón simple

Ing. María Paula Zappitelli 
Copyright (C) María Paula Zappitelli 
A mi familia y amigos 

Nada en el mundo sustituye a la constancia. El talento no la sustituye, pues nada es tan corriente como los inteligentes frustrados. El genio tampoco, ya que resulta ser tópico el caso de los genios ignorados. Ni siquiera la educación sustituye a la constancia, pues el mundo está lleno de fracasados bien educados. Solamente la constancia y la decisión lo consiguen todo. 



\section{Agradecimientos}

En primer lugar quiero agradecerles a mis directores, los Drs. Claudio Rocco e Ignacio Villa por haberme introducido en el camino de la investigación, por la confianza brindada a lo largo de estos años y por sus valiosos aportes, dedicación y apoyo.

Al Prof. Dr. José Fernández Sáez de la Universidad Carlos III de Madrid, por haberme co-dirigido en la beca doctoral que me otorgó la Universidad Nacional de La Plata.

Al Departamento de Construcciones, mi lugar de trabajo desde hace diez años, y a mis compañeros del Área Mecánica Computacional con los que compartí tanto los días buenos como los malos a lo largo de la Tesis, cuando los modelos no convergían, los códigos no compilaban o cada vez que conseguía un buen resultado.

A las autoridades del LEMIT, a los integrantes del Área de Estudios Especiales, en donde se desarrolló el programa experimental de la Tesis y en particular al Dr. Raúl Zarbino, la Ing. Graciela Giaccio y la Dr. Celeste Torrijos, por el tiempo, la dedicación y los conocimientos que compartieron conmigo.

A Hormicova S.A. por facilitar el cemento para los ensayos realizados.

A mis amigos por alegrarse con mis progresos y alentarme en los momentos complicados para seguir adelante, por escucharme e interesarse.

A mi familia, en particular a mis abuelos y mi tía Celia, que aunque ya no están me transmitieron el valor del esfuerzo, la responsabilidad y las ganas de superarme. A mis hermanos José Luis y Juan Marcos, y especialmente a mi papá Juan José y mi mamá Liliana por acompañarme, incentivar mis ganas de aprender y apoyarme siempre en mis proyectos, por alegrarse con mis logros y por tener la palabra justa en los momentos complicados para no bajar los brazos y seguir intentando.

Finalmente quiero agradecerle a Seba por haberme acompañado en la última etapa de este proceso, pero no por eso menos importante.

Esta Tesis es para todos ustedes. 



\section{Resumen}

Cuando la contracción térmica y la contracción por secado superan ciertos valores límites en las estructuras de hormigón, sumado a las condiciones de restricción a las que se encuentran sometidas dichas estructuras, usualmente las mismas se fisuran. En aquellos casos en los que no se encuentra comprometida la estabilidad estructural poder controlar el fenómeno de fisuración es importante porque la vida en servicio de las estructuras puede encontrarse afectada. Por esta razón, resulta de interés predecir la edad y los patrones de fisuración, pero esto no es frecuentemente posible en estructuras sin defectos o sometidas a estados tensionales uniformes, resultando necesario implementar un criterio para definir zonas de fisuración preferentes.

En la presente tesis se propone un criterio para definir dichas zonas de fisuración preferentes basado en la heterogeneidad del hormigón, resultado de sus múltiples fases componentes, las cuales originan propiedades mecánicas no uniformes en el material. Para considerar la heterogeneidad del hormigón se desarrolló un algoritmo basado en números aleatorios, que incluye la variación de las propiedades mecánicas del material en el tiempo. El algoritmo se aplica en un código de elementos finitos y agrupa a los elementos de la malla de elementos finitos en sets asignándoles una resistencia diferente mediante la utilización de números aleatorios siguiendo una distribución de Gauss o de Weibull. Para la validación del algoritmo se reprodujeron resultados experimentales disponibles en la bibliografía de ensayos de anillos circulares y elípticos sometidos a contracción restringida y ensayos propios en anillos circulares utilizando un modelo de plasticidad con daño. Las edades y patrones de fisuración obtenidos muestran buena correlación con los resultados de los ensayos. 



\section{Índice}

Agradecimientos $\quad$ IX

Resumen $\quad$ XI

1. Introducción 1

1.1. Motivación . . . . . . . . . . . . . . 1

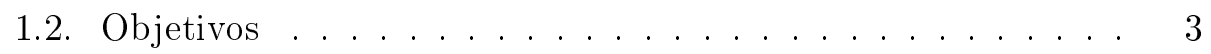

1.3. Organización del documento . . . . . . . . . . . . . 4

1.4. Aportes originales de la Tesis . . . . . . . . . . . . 4

2. Antecedentes 7

2.1. Introducción . . . . . . . . . . . . . 7

2.2. Fenómenos de cambio de volumen en el hormigón endurecido 8

2.2.1. Contracción . . . . . . . . . . . . . . . 9

2.2.1.1. Contracción plástica . . . . . . . . . . 9 9

2.2.1.2. Contracción química . . . . . . . . . . . 10

2.2.1.3. Contracción autógena . . . . . . . . . . 12

2.2.1.4. Contracción por secado . . . . . . . . . 13

2.2.1.5. Contracción térmica . . . . . . . . . . 17

2.2.1.6. Contracción por carbonatación . . . . . . 2 20

2.2.2. Expansión . . . . . . . . . . . . . . 20

2.2.3. Creep . . . . . . . . . . . . . . . 21

2.2.4. Cambios de volumen debidos a efectos químicos . . . . 22

2.2.4.1. Ataque por sulfatos . . . . . . . . . . . 22

2.2.4.2. Reacciones álcali-agregado . . . . . . . . 22

2.3. Modelación numérica del comportamiento mecánico del hormigón ......................... 23

2.3.1. Modelos para el estudio del comportamiento del hormigón bajo carga . . . . . . . . . . . . . . 28

2.3.1.1. Mecánica de Fractura Elástica Lineal (MFEL) 29

2.3.1.2. Modelo de Fisura Cohesiva (MFC) . . . . . . 31

2.3.1.3. Modelos de plasticidad con daño . . . . . . . 37 


\subsubsection{Método de los Elementos Finitos Extendido} (XFEM)

3. Algoritmo desarrollado y modelo numérico aplicado para el estudio del proceso de fisuración en estructuras de hormigón 45

3.1. Introducción . . . . . . . . . . . . . . . . 45

3.2. Algoritmo desarrollado para la asignación aleatoria de propiedades mecánicas del hormigón . . . . . . . . . . . . . 48

3.3. Selección del modelo numérico . . . . . . . . . . . . . . . 52

3.4. Aplicaciones del algoritmo desarrollado . . . . . . . . . . 75

3.4.1. Ensayo de tracción directa . . . . . . . . . . . . 76

3.4.2. Elemento de hormigón sometido a contracción uniforme 79

3.4.3. Elementos de hormigón sometidos a gradientes de temperatura . . . . . . . . . . . . 84

4. Programa experimental 95

4.1. Introducción . . . . . . . . . . . . . . . . 95

4.2. Etapa preliminar . . . . . . . . . . . . . . . 96

4.3. Ensayos de caracterización . . . . . . . . . . . . . . . 101

4.3.1. Tiempo de fraguado . . . . . . . . . . . . . 101

4.3.2. Curva de elevación adiabática de la temperatura del hormigón . . . . . . . . . . . . . . 103

4.3.3. Resistencia a la compresión . . . . . . . . . . . . . 104

4.3.4. Resistencia a la tracción por compresión diametral . . 106

4.3.5. Módulo de elasticidad estático . . . . . . . . . . . . . 109

4.3.6. Módulo de elasticidad dinámico . . . . . . . . . . . 111

4.3.7. Ensayo de contracción libre . . . . . . . . . . . . 113

4.3.8. Determinación de la energía de fractura en Modo I . . 118

4.3.9. Ensayo de extensibilidad en tracción . . . . . . . . . 122

4.4. Ensayos de validación . . . . . . . . . . . . . . . . 125

4.4.1. Ensayo de contracción restringida en anillos sin entallas 125

4.4.2. Ensayo de contracción restringida en anillos con entallas130

5. Validación numérica del algoritmo desarrollado 139

5.1. Introducción . . . . . . . . . . . . . . . . . 139

5.2. Validación del algoritmo desarrollado considerando resultados experimentales propios . . . . . . . . . . . . 139

5.3. Validación del algoritmo desarrollado a partir de los resultados de otros autores . . . . . . . . . . . . . . 146

5.3.1. Determinación de la edad de fisuración . . . . . . . . . 149

5.3.2. Zonas dañadas . . . . . . . . . . . . . . . . . 152 
6. Conclusiones y Trabajo Futuro 157

6.1. Conclusiones . . . . . . . . . . . . . 157

6.2. Trabajo futuro . . . . . . . . . . . . . 159

$\begin{array}{ll}\text { Bibliografía } & 161\end{array}$

Ing. M. Paula Zappitelli $\quad$ XV 



\section{Índice de figuras}

2.1. Fisuras por restricción externa, CIRIA-660 (2007) (izquierda), fisuras por restricción interna (derecha) . . . . . . . . 8

2.2. Mecanismo de la contracción plástica en el hormigón, Schmidt y Slowik (2013) . . . . . . . . . . . . . . . 11

2.3. Cambios de volumen debidos a contracción química y autógena en la pasta de cemento fresca y endurecida, Kosmatka et al. $(2002) \ldots \ldots \ldots \ldots \ldots$

2.4. Mecanismo de la contracción por secado, Radocea (1992) . . . 14

2.5. Factores que afectan la contracción por secado, Mindess et al.

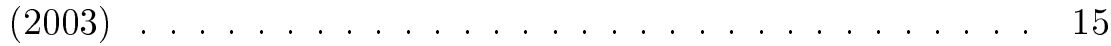

2.6. Reversibilidad e irreversibilidad de la contracción en el hormigón sumergido en agua y luego expuesto al aire comparada con la expansión sufrida por un hormigón continuamente sumergido en agua, Neville (2011) . . . . . . . . . . 18

2.7. Escalas de análisis en el hormigón, micro escala (izquierda), meso escala (centro), macro escala (derecha),van Mier (1997)

2.8. Clasificación de los métodos para representar fisuras en modelos numéricos, Ingraffea (2007) . . . . . . . . . . . . 29

2.9. Modos de fractura, Kumar y Barai (2011) . . . . . . . . . . . 31

2.10. (a) Ensayo de tracción uniaxial en una probeta de hormigón. (b) Curva tensión-elongación con una sola fisura. (c) Fisura suavizada perpendicular al eje de la probeta (d) Curva tensión-elongación en un ensayo de tracción estable, Baẑant y Planas (1997) . . . . . . . . . . . . . . . 33

2.11. Ensayo de tracción idealizado de una probeta de hormigón, Baẑant y Planas (1997) . . . . . . . . . . . . . 34

2.12. Curva de ablandamiento con comportamiento lineal hasta la carga pico, Bažant y Planas (1997) . . . . . . . . . . 35

2.13. Tensión cohesiva vs apertura de fisura para el modelo original de Dugdale y para el ablandamiento rectangular, Baẑant y Planas (1997) .................. 36 
2.14. Curvas de ablandamiento rectangular, lineal y bilineal de Peterson, Baẑant y Planas (1997) . . . . . . . . . . 37

2.15. Coordenadas normal y tangencial en una fisura aplicando método XFEM, ABAQUS (2013a) . . . . . . . . . . 42

2.16. Principio del Método de los nodos fantasma, ABAQUS (2013a) 44

3.1. Función densidad de probabilidad de Gauss y Weibull (izquierda). Comparación entre las distribuciones acumuladas de Gauss y Weibull (derecha) . . . . . . . . . . . . . . 47

3.2. Malla de elementos finitos convencional (izquierda). Malla de material (derecha) utilizadas por el algoritmo desarrollado . . 49

3.3. Superposición de la malla de material y la malla de elementos finitos . . . . . . . . . . . . . . . 49

3.4. Método de la inversa acumulada para obtener números aleatorios con una distribución de probabilidad distinta de la uniforme 51

3.5. Asignación de propiedades mecánicas en una geometría elíptica utilizando el algoritmo desarrollado . . . . . . . . . 52

3.6. Diagrama de flujo que describe el funcionamiento del algoritmo desarrollado . . . . . . . . . . . . . 53

3.7. Representación de un elemento cohesivo, ABAQUS (2013c) . 55

3.8. Direcciones de las tensiones nominales en un elemento cohesivo 56

3.9. Malla de elementos finitos del bloque estudiado para la aplicación del Modelo de Fisura Cohesiva-Elementos cohesivos . . 58

3.10. Patrón de fisuración obtenido aplicando elementos cohesivos para el bloque estudiado en el análisis comparativo de modelos numéricos . . . . . . . . . . . . . . . 59

3.11. Superficie de fluencia en el plano desviador correspondiente a diferentes valores de $\mathbf{K}_{c}$, ABAQUS (2013b) . . . . . . 63

3.12. Superficie de fluencia en tensión plana, ABAQUS (2013b) . . 64

3.13. Respuesta del hormigón bajo carga uniaxial en tracción (a) y compresión (b), ABAQUS (2013b) . . . . . . . . . 66

3.14. Definición de la deformación por fisuración utilizada para definir el endurecimiento en tracción, ABAQUS (2013b) . . . . 67

3.15. Definición de la deformación inelástica en compresión usada para el cálculo del endurecimiento en compresión . . . . . . 68

3.16. Malla de elementos finitos del bloque modelado para el análisis comparativo de los modelos numéricos aplicados al estudio del proceso de fisuración en hormigón . . . . . . . . . . . . 70

3.17. Vectores representativos de la deformación principal máxima para el bloque estudiado en el análisis comparativo de modelos utilizando el modelo CDP . . . . . . . . . . . . . . 72 
3.18. Representación de la variable de daño para el bloque estudiado en el análisis comparativo de modelos utilizando el modelo CDP 72

3.19. Patrón de fisuración obtenido aplicando el método XFEM para el bloque estudiado en el análisis comparativo de modelos numéricos para el estudio del proceso de fisuración . . . . . . 74

3.20. Mallado y condición de carga de la probeta de 10 x 20 x 50 cm sometida al ensayo de tracción directa . . . . . . . 76

3.21. Curva carga-desplazamiento del punto de aplicación de la carga en el ensayo de tracción directa para cada uno de los casos analizados . . . . . . . . . . . . . . 77

3.22. Curvas tensión-desplazamiento para cada uno de los casos

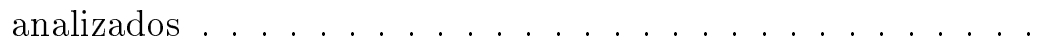

3.23. Zonas dañadas correspondientes a la probeta de $10 \times 20 \times 50$ $\mathrm{cm}$ simulada considerando distribución uniforme de las propiedades mecánicas . . . . . . . . . . . . . .

3.24. Zonas dañadas correspondientes a la probeta de $10 \times 20 \times 50$ $\mathrm{cm}$ simulada considerando distribución aleatoria de las propiedades mecánicas . . . . . . . . . . . . . .

3.25. Mallados utilizados en la modelación del bloque analizado con el objetivo de estudiar la independencia de malla aplicando el algoritmo desarrollado . . . . . . . . . . . . . 80

3.26. Distribuciones aleatorias de propiedades mecánicas para el bloque analizado ................. 80

3.27. Zonas dañadas para el bloque de hormigón sometido a una contracción térmica uniforme con distribución uniforme de las propiedades mecánicas y mallado cuadrado . . . . . . . . . 84

3.28. Zonas dañadas para el bloque de hormigón sometido a una contracción térmica uniforme con distribución uniforme de las propiedades mecánicas y mallado triangular . . . . . . . . . 84

3.29. Zonas dañadas para el bloque de hormigón sometido a una contracción térmica uniforme con distribución aleatoria de las propiedades mecánicas y mallado cuadrado . . . . . . . . .

3.30. Zonas dañadas para el bloque de hormigón sometido a una contracción térmica uniforme con distribución aleatoria de las propiedades mecánicas y mallado triangular . . . . . . . 8 85

3.31. Curva de elevación de temperatura adiabática utilizada en los análisis térmicos para el estudio de la fisuración por contracción térmica debido a gradientes de temperatura . . . . . . 86

3.32. Evolución de temperatura en el elemento de $10 \times 50 \mathrm{~cm}$ biempotrado, edades 1 h, 10 hs, 24 hs y 28 días . . . . . . . 87

3.33. Evolución de temperatura en elemento de 50 x $250 \mathrm{~cm}$ biempotrado, edades $1,3,7$ y 28 días . . . . . . . . . .

Ing. M. Paula Zappitelli 
3.34. Evolución de temperatura en elemento de 50 x $50 \mathrm{~cm}$ biempotrado, edades $1,3,7$ y 28 días . . . . . . . . 88

3.35. Variable de daño para el elemento de $10 \times 50 \mathrm{~cm}$ biempotrado simulado con propiedades mecánicas uniformes . . . . . . 90

3.36. Variable de daño para el elemento de $10 \times 50 \mathrm{~cm}$ biempotrado simulado con propiedades mecánicas aleatorias . . . . . . . 90

3.37. Evolución del crecimiento de la zona dañada . . . . . . . . . . 91

3.38. Variable de daño para el elemento de 50 x $250 \mathrm{~cm}$ biempotrado simulado con propiedades mecánicas uniformes . . . . . . 92

3.39. Variable de daño para el elemento de $50 \times 250 \mathrm{~cm}$ biempotrado simulado con propiedades mecánicas aleatorias . . . . . . . 92

3.40. Variable de daño para la geoemtría de 50 x $50 \mathrm{~cm}$ biempotrada simulada con propiedades mecánicas uniformes . . . . . . . 93

3.41. Variable de daño para la geometría de 50 x $50 \mathrm{~cm}$ biempotrada simulada con propiedades mecánicas aleatorias . . . . . . 94

4.1. Proceso de hormigonado y llenado de probetas . . . . . . 97

4.2. Ensayo de anillo de contracción restringida según norma ASTM C1581. Molde y anillo de acero (izquierda) probeta (derecha)

4.3. Equipo de registro de deformaciones para el ensayo de contracción restringida conectado a los anillos según norma ASTM $\mathrm{C} 1581 \ldots \ldots \ldots \ldots \ldots \ldots \ldots$

4.4. Registros de deformaciones para los anillos moldeados con el hormigón 1 (400 $\mathrm{Kg} / \mathrm{m}^{3}$ de cemento), el hormigón 2 (500 $\mathrm{Kg} / \mathrm{m}^{3}$ de cemento) y el hormigón $3\left(600 \mathrm{Kg} / \mathrm{m}^{3}\right.$ de cemento) en la etapa preliminar . . . . . . . . . . . . . 100

4.5. Ensayo para la determinación del tiempo de fraguado para un hormigón con un contenido de cemento de $600 \mathrm{Kg} / \mathrm{m}^{3} \ldots$. . . 102

4.6. Medición tiempo de fraguado para el hormigón de $600 \mathrm{Kg} / \mathrm{m}^{3}$ de cemento . . . . . . . . . . . . . . 103

4.7. Registrador de temperatura . . . . . . . . . . . . . 104

4.8. Curva de elevación adiabática para el hormigón estudiado . . 104

4.9. Ensayo de compresión en probetas cilíndricas de 10 x $20 \mathrm{~cm} \cdot 105$

4.10. Evolución de la resistencia a compresión en el tiempo y curva de ajuste . . . . . . . . . . . . 106

4.11. Aplicación de cargas en el ensayo de tracción por compresión diametral . . . . . . . . . . . . . . . 107

4.12. Ensayo de tracción por compresión diametral en probetas cilíndricas de $10 \times 20 \mathrm{~cm}$. . . . . . . . . . . . . 107

4.13. Evolución de la resistencia a tracción en el tiempo y curva de ajuste ..................... 108 
4.14. Compresómetro utilizado para el ensayo del módulo de elasticidad estático en probetas cilíndricas de $10 \times 20 \mathrm{~cm}$. . . 109

4.15. Evolución módulo de elasticidad estático en el tiempo y curva de ajuste. . . . . . . . . . . . . . 110

4.16. Ensayo para la determinación del módulo de elasticidad dinámico . . . . . . . . . . . . . . . . 111

4.17. Evolución módulo de elasticidad dinámico en el tiempo y curva de ajuste . . . . . . . . . . . . . . . 112

4.18. Prueba de pinturas de aislación . . . . . . . . . . . 114

4.19. Evolución en el tiempo de la pérdida de peso en las probetas con cada tipo de pintura de aislación . . . . . . . . . . . 114

4.20. Ensayo de contracción libre en probetas prismáticas de $7 \times 7$ x $30 \mathrm{~cm} \ldots \ldots \ldots \ldots \ldots \ldots \ldots$

4.21. Evolución contracción libre en el tiempo para cada condición de aislación . . . . . . . . . . . . . . 116

4.22. Temperaturas equivalentes obtenidas a partir del ensayo de contracción libre . . . . . . . . . . . . . . 117

4.23. Relaciones $A / V$ para cada una de las edades analizadas . . . 117

4.24. Curva de ajuste para el cálculo de las temperaturas equivalentes en el anillo de hormigón . . . . . . . . . . . . . 118

4.25. Ensayo para la determinación de la energía específica de fractura en modo I . . . . . . . . . . . . . . . . . . . . 119

4.26. Esquema de compensación de pesos para el ensayo de energía de fractura . . . . . . . . . . . . . . . . 119

4.27. Curva carga-flecha para el ensayo de energía de fractura . . . 120

4.28. Curva carga-CMOD para el ensayo de energía de fractura . . 121

4.29. Estimación de la evolución de la energía de fractura en el tiempo121

4.30. Ensayo de extensibilidad a carga lenta . . . . . . . . . 123

4.31. Ensayo de extensibilidad a carga rápida . . . . . . . . . . 123

4.32. Resultados de los ensayos de extensibilidad a carga rápida y lenta . . . . . . . . . . . . . . . . . . 124

4.33. Ajuste del módulo de elasticidad considerando el efecto del creep . . . . . . . . . . . . . . 125

4.34. Deformaciones medidas en los anillos de contracción restringida durante los primeros días . . . . . . . . . . . . 126

4.35. Registros de deformaciones para el ensayo de contracción restringidaAnillo $1 \ldots \ldots \ldots$. . . . . . . . . . . 127

4.36. Registros de deformaciones para el ensayo de contracción restringidaAnillo $2 \ldots \ldots \ldots \ldots$. . . . . . . . . . 127

4.37. Registros de deformaciones para el ensayo de contracción restringidaAnillo $3 \ldots \ldots \ldots \ldots$. . . . . . . . . . . . . . . . . . . . 
4.38. Registros de deformaciones para el ensayo de contracción restringidaAnillo $4 \ldots \ldots \ldots \ldots$. . . . . . . . . . 128

4.39. Registros de deformaciones para el ensayo de contracción restringida-

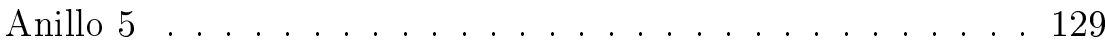

4.40. Registros de deformaciones para el ensayo de contracción restringidaAnillo 6 . . . . . . . . . . . . . . . . . . 129

4.41. Ensayo de contracción restringida en anillos de hormigón sin entalla-Fisuras observadas . . . . . . . . . . . . . . 130

4.42. Anillos entallados . . . . . . . . . . . . . . . . . 131

4.43. Registros de deformaciones para el ensayo de contracción restringidaAnillo 3 sin entalla . . . . . . . . . . . . . . . . . . 132

4.44. Registros de deformaciones para el ensayo de contracción restringidaAnillo 4 entallado . . . . . . . . . . . . . . . . . 132

4.45. Registros de deformaciones para el ensayo de contracción restringidaAnillo 5 entallado . . . . . . . . . . . . . . . . 133

4.46. Registros de deformaciones para el ensayo de contracción restringidaAnillo 6 entallado . . . . . . . . . . . . . . . . . . 133

4.47. Ensayo de contracción restringida en anillos de hormigón con entalla- Fisuras observadas . . . . . . . . . . . . . . 134

4.48. Proceso de calibración del microscopio digital utilizado para la medición de la apertura de las fisuras . . . . . . . . . 135

4.49. Apertura de la entalla medida en el lateral del anillo 4 . . . 136

4.50. Apertura de la entalla medida en el lateral del anillo 5 . . . 136

4.51. Apertura de la entalla medida en el lateral del anillo 6 . . . 137

4.52. Apertura de la fisura medida en el lateral del anillo 3 . . . . 137

4.53. Fotografía para la medición de la apertura de la entalla en anillos 4 y $6 \ldots \ldots \ldots \ldots \ldots$

5.1. Mallas utilizadas en la simulación del ensayo de contracción restringida en anillos circulares . . . . . . . . . . . . . 140

5.2. Elementos del anillo de acero en los que se miden las deformaciones circunferenciales . . . . . . . . . . . . . . . 141

5.3. Variación de la deformación circunferencial en el tiempo para la simulación del ensayo de contracción restringida en anillos de hormigón . . . . . . . . . . . . . . . . . . . . 142

5.4. Comparación edades de fisuración experimental y numéricaAnillo 4 con entalla . . . . . . . . . . . . . . . . . . . . . 145

5.5. Comparación edades de fisuración experimental y numéricaAnillo 6 con entalla . . . . . . . . . . . . . . . . . . . . 145

5.6. Comparación edades de fisuración experimental y numéricaAnillo 3 sin entalla . . . . . . . . . . . . . . . . . 146

5.7. Geometría de los anillos analizados . . . . . . . . . . 146 
5.8. Mallados utilizados en los anillos elípticos analizados . . . . 147

5.9. Temperatura equivalente utilizada en las simulaciones, Dong et al. (2016) . . . . . . . . . . . . . . . . 149

5.10. Ubicación de las fisuras en ensayos experimentales para el anillo 2, Dong et al. (2016) . . . . . . . . . . . . . . 152

5.11. Zonas dañadas para simulaciones realizadas con mallado cuadrado y material aleatorio para el anillo $2 \ldots$. . . . . . 153

5.12. Zonas dañadas para simulaciones realizadas con mallado trian-

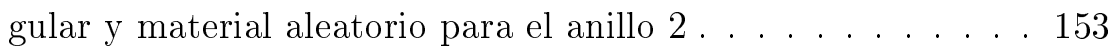

5.13. Ubicación de las fisuras en ensayos experimentales para el anillo 3, Dong et al. (2016) . . . . . . . . . . . . . 154

5.14. Zonas dañadas para las simulaciones realizadas con mallado cuadrado y material aleatorio para el anillo $3 \ldots . . . . .155$

5.15. Zonas dañadas para las simulaciones realizadas con mallado triangular y material aleatorio para el anillo $3 \ldots . . . .155$ 



\section{Índice de Tablas}

3.1. Propiedades mecánicas del hormigón utilizado en el análisis comparativo de los diferentes modelos numéricos para el estudio del proceso de fisuración . . . . . . . . . . . . . 54

3.2. Parámetros del modelo de plasticidad con daño utilizado en el análisis comparativo de los diferentes modelos numéricos para el estudio del proceso de fisuración . . . . . . . . . . . 70

3.3. Modelo de plasticidad con daño considerando un mallado cuadrado: tiempo de aparición de las fisuras . . . . . . . . . . 71

3.4. Modelo de plasticidad con daño considerando un mallado triangular: tiempo de aparición de las fisuras . . . . . . . . 71

3.5. Carga de rotura y resistencia a tracción para el ensayo de tracción directa obtenidas aplicando distribución aleatoria de las propiedades mecánicas . . . . . . . . . . . 77

3.6. Edad de fisuración para un bloque con distribución de propiedades mecánicas aleatoria para una malla cuadrada con elementos de $50 \mathrm{~mm}$ de lado . . . . . . . . . . . . 81

3.7. Edad de fisuración para un bloque con distribución de propiedades mecánicas aleatoria para una malla cuadrada con elementos de $100 \mathrm{~mm}$ de lado . . . . . . . . . . . 82

3.8. Edad de fisuración para un bloque con distribución de propiedades mecánicas aleatoria para una malla triangular con tamaño de elementos de $50 \mathrm{~mm}$ de lado . . . . . . . . . 83

3.9. Edad de fisuración para un bloque con distribución de propiedades mecánicas aleatoria para una malla triangular con tamaño de elementos de $100 \mathrm{~mm}$ de lado . . . . . . . . . 83

3.10. Edad de fisuración para un elemento de $10 \times 50 \mathrm{~cm}$ biempotrado con distribución de propiedades mecánicas aleatoria sometida a gradientes de temperatura . . . . . . . . 89

3.11. Edad de fisuración para el elemento biempotrado de $50 \times 250$ $\mathrm{cm}$ con distribución de propiedades mecánicas aleatoria sometido a gradientes de temperatura . . . . . . . . . . . . 91 
3.12. Edad de fisuración para la geometría de 50 x $50 \mathrm{~cm}$ biempotrada con distribución de propiedades mecánicas aleatorias sometido a gradientes de temperatura $\ldots \ldots \ldots . \ldots 93$

4.1. Dosificaciones de los hormigones de la etapa preliminar . . . . 96

4.2. Propiedades en estado fresco de los hormigones analizados en la etapa preliminar . . . . . . . . . . . . . 96

4.3. Edad de fisuración de los anillos analizados en la etapa preliminar . . . . . . . . . . . . . . . 99

4.4. Resultados ensayos de resistencia a la compresión de los hormigones 1, 2 y 3 a la edad de fisuración en la etapa preliminar 100

4.5. Resultados ensayos de resistencia a la tracción por compresión diametral de los hormigones 1,2 y 3 a la edad de fisuración en la etapa preliminar . . . . . . . . . . . . . . . 101

4.6. Resultados ensayos de módulo de elasticidad estático de los hormigones 1, 2 y 3 a la edad de fisuración en la etapa preliminar101

4.7. Resultados de los ensayos de resistencia a la compresión en probetas cilíndricas de $10 \times 20 \mathrm{~cm}$. . . . . . . . 105

4.8. Resultados de los ensayos de resistencia a la tracción por compresión diametral . . . . . . . . . . . . . . . . 108

4.9. Resultados ensayos de módulo de elasticidad estático . . . . 110

4.10. Resultados ensayos de módulo de elasticidad dinámico . . . . 112

4.11. Relaciones A/V para probetas de $7 \times 7 \times 30 \mathrm{~cm}$ bajo distintas condiciones de aislación . . . . . . . . . . . 116

4.12. Cálculo de energía de fractura a 28 días . . . . . . . . . 120

4.13. Registro visual del ensayo de contracción restringida en anillos sin entallas. FS (fisura superficial), SC(sin cambios), F(fisura) 126

4.14. Tiempos de aparición de fisuras obtenidos a partir de los registros continuos-Anillos sin entalla . . . . . . . . 130

4.15. Registro visual del ensayo de contracción restringida en anillos con entallas. FS (fisura superficial), SC(sin cambios), F(fisura) 131

4.16. Tiempos de aparición de fisuras obtenidos a partir de los registros continuos- Anillos entallados . . . . . . . . . . . 134

5.1. Tiempo de aparición de la fisura en anillos circulares con mallado cuadrado . . . . . . . . . . . . . . . . . . 142

5.2. Tiempo de aparición de la fisura en anillos circulares con mallado triangular . . . . . . . . . . . . . . . 143

5.3. Comparación resultados experimentales y numéricos para el mallado cuadrado . . . . . . . . . . . . . . . . 143

5.4. Comparación resultados experimentales y numéricos para el mallado triangular . . . . . . . . . . . . . . . 143 
5.5. Tiempo de aparición de la fisura en anillos circulares entallados simulados numéricamente . . . . . . . . . . . . . 144

5.6. Comparación resultados experimentales y numéricos para el anillo entallado . . . . . . . . . . . . . . . 144

5.7. Dimensiones de los anillos circulares y elípticos analizados . . 147

5.8. Tamaños de elementos utilizados para analizar su efecto en los resultados obtenidos-Mallado cuadrado . . . . . . . . . 147

5.9. Tamaños de elementos utilizados para analizar su efecto en los resultados obtenidos-Mallado triangular . . . . . . . 147

5.10. Edad de fisuración obtenida en simulaciones realizadas en anillos con mallado cuadrado-Mallado fino . . . . . . . . . . . 150

5.11. Edad de fisuración obtenida en simulaciones realizadas en anillos con mallado triangular-Mallado fino . . . . . . . . . 150

5.12. Edad de fisuración obtenida en simulaciones realizadas en anillos con mallado cuadrado-Mallado grueso . . . . . . . . . 151

5.13. Edad de fisuración obtenida en simulaciones realizadas en anillos con mallado triangular-Mallado grueso . . . . . . . . . 151 



\section{Capítulo 1}

\section{Introducción}

\subsection{Motivación}

El hormigón, constituido por agregados embebidos en una matriz de pasta de cemento, es uno de los materiales de construcción más empleados por el hombre para la ejecución de obras civiles. Las razones por las que se lo utiliza comprenden su capacidad para soportar la acción deletérea del agua, la facilidad con la que pueden moldearse elementos estructurales de variadas formas y tamaños y constituye el material más económico y más rápidamente disponible en las obras, Metha y Monteiro (1998). Es interesante destacar que el hormigón experimenta cambios de volumen frente a dos fenómenos que son inherentes a su propia naturaleza. Uno es la contracción asociada con la pérdida de humedad que se produce dentro de la compleja red de poros que integran la pasta de cemento hidratada, fenómeno que da lugar a la contracción por secado y a la contracción autógena, y el otro es el cambio de volumen debido a las variaciones de temperatura provocadas por el calor liberado durante el proceso de hidratación del cemento que genera un calentamiento inicial seguido de su posterior enfriamiento. Este último fenómeno se denomina contracción térmica. Como consecuencia de ambos tipos de contracción se producen en las estructuras de hormigón deformaciones de magnitud variable que inducen tensiones en el material cuyo nivel depende del gradiente del campo de deformaciones, de la magnitud de las mismas y del grado de restricción impuesto por las condiciones de vínculo de la estructura, Metha y Monteiro (1998).

Las tensiones producidas por la contracción por secado y/o los cambios de volumen de origen térmico son una de las principales causas de fisuración de las estructuras de hormigón, ya sean masivas o convencionales, simples o armadas, con un impacto económico muy importante en los costos de reparación y mantenimiento. Si bien en los reglamentos de aplicación al proyecto y construcción de dichas estructuras se establecen pautas para la prevención o el control de este tipo de fisuras, en general se trata de medidas de orden 
prescriptivo, de naturaleza empírica, que no permiten contemplar de manera explícita al conjunto de variables que intervienen en el fenómeno y que en consecuencia limitan la posibilidad de optimizar, con criterio racional, la respuesta de la estructura ante estas acciones.

En el contexto planteado, la ventaja de disponer de modelos de aplicación a escala estructural que sean capaces de estudiar las acciones producidas por la contracción por secado y/o el calor liberado durante la hidratación del cemento y que tengan además la capacidad de describir o capturar el proceso de aparición y propagación de fisuras inherente a estos fenómenos, se presenta como una herramienta de sumo interés para el estudio y análisis de esta problemática y como una herramienta de gran utilidad para poder optimizar, desde una perspectiva más racional, la selección del material, el diseño estructural y los procedimientos constructivos.

Para modelar el proceso de fisuración en estructuras de hormigón sometidas a estados tensionales uniformes es necesario identificar zonas de fisuración preferentes. En algunos casos esto es sencillo debido a cuestiones geométricas o por la presencia de defectos, pero cuando esto no ocurre es necesario incorporar un criterio para lograrlo. Una posibilidad consiste en considerar la heterogeneidad presente en el hormigón, la cual es producto de sus diferentes fases componentes.

Para incorporar dicha heterogeneidad a los modelos numéricos en la bibliografía se plantean dos enfoques: directo e indirecto, Yang et al. (2009). En el primer caso se modelan todas las fases del hormigón a nivel de meso escala (matriz de cemento, agregados e interfases), asignando a cada una de ellas sus correspondientes propiedades mecánicas. En este tipo de modelos la distribución de los agregados y las interfases se realiza a partir de imágenes obtenidas por tomografía computada o utilizando un algoritmo que permite distribuir los agregados aleatoriamente, considerando distintos tipos de geometrías para los mismos (circular, elíptica, poligonal), Chen et al. (2018). Por otro lado, en el enfoque indirecto, se considera la variación espacial de las propiedades mecánicas del material, tales como la resistencia a la tracción y compresión, lo cual puede modelarse, por un lado en el marco del Método de los Elementos Finitos Estocásticos (SFEM, por sus siglas en inglés) Stefanou (2009) o considerando otros métodos.

SFEM se define como un método que tiene en cuenta las incertidumbres presentes en la geometría o el material de una estructura, así como en las cargas actuantes, combinado con la discretización del dominio analizado utilizando el Método de los Elementos Finitos (MEF), Der Kiureghian y Ke (1988).

Los otros métodos consideran la utilización de números aleatorios vinculados a cada uno de los elementos de la malla de elementos finitos, para asignar las propiedades mecánicas o métodos de disipación local de energía.

Una vez definido el criterio para determinar las zonas de fisuración pre- 
ferentes, es necesario seleccionar un modelo numérico de fractura en donde incorporarlo con la finalidad de aplicarlo a la resolución de problemas concretos de fisuración. Los modelos a utilizar se dividen en dos grandes grupos, a partir de la forma en que se representan las fisuras: representación geométrica y representación no geométrica, Ingraffea (2007).

En los primeros la fisura es una entidad geométrica, por lo que el modelo geométrico y el modelo discretizado se actualizan con el crecimiento de la misma. La Mecánica de Factura Elástica Lineal y el Modelo de Fisuración Cohesiva, mediante la aplicación de elementos o superficies cohesivas, puede enmarcase en este grupo.

Por otro lado, los modelos de representación no geométrica no contienen a la fisura y la discretización no cambia durante el crecimiento de la misma. En este caso la fisura se encuentra representada, ya sea en el modelo constitutivo o en un modelo cinemático, en base a las deformaciones. Ejemplos de este tipo de modelos los constituyen los modelos de plasticidad con daño y el Método de los Elementos Finitos Extendido (XFEM).

Por lo expuesto anteriormente, resulta de interés contar con un criterio, que basado en la representación numérica de la heterogeneidad inherente al hormigón como material estructural, y aplicado a un Modelo de Elementos Finitos, permita predecir fenómenos de fisuración por contracción en estructuras de hormigón.

\subsection{Objetivos}

El objetivo principal de la presente Tesis consiste en la simulación numérica de fenómenos de fisuración por contracción en estructuras de hormigón mediante modelos no lineales, investigando su capacidad predictiva tanto en relación con el momento de aparición de las fisuras como con los patrones de fisuración obtenidos experimentalmente.

Para la consecución del objetivo propuesto se plantea la necesidad de cumplir los objetivos específicos que se indican a continuación:

- Evaluar las bondades y dificultades de los modelos numéricos existentes.

- Proponer y desarrollar algoritmos y subrutinas que permitan la asignación aleatoria de propiedades mecánicas y de fractura, considerando su variación en el tiempo.

- Implementar y calibrar un modelo numérico no lineal para determinar la localización y el tiempo de aparición de fisuras en estructuras de hormigón simple sometidas a fenómenos de contracción.

- Validar el modelo numérico implementado utilizando para ello resultados numéricos, analíticos y experimentales.

Ing. M. Paula Zappitelli 


\subsection{Organización del documento}

La presente Tesis doctoral se encuentra estructurada en seis capítulos. El primero de ellos lo constituye esta introducción, en donde se pone de manifiesto el tema estudiado, su interés y los objetivos planteados. En el capítulo 2 se realiza una breve recopilación bibliográfica acerca del fenómeno de fisuración por contracción en el hormigón, la modelación numérica del hormigón como material y los modelos numéricos propuestos para estudiar el proceso de fisuración en estructuras de hormigón.

En el capítulo 3 se describe el algoritmo desarrollado para la asignación aleatoria de las propiedades mecánicas del hormigón, se justifica la adopción del modelo de plasticidad con daño, para el estudio del fenómeno de fisuración por contracción y se presenta la implementación del algoritmo desarrollado en el mencionado modelo. Para ello se analizan diferentes casos: un ensayo de tracción directa sobre una probeta de hormigón, un bloque de hormigón sometido a contracción térmica uniforme y elementos de hormigón con diferente relación de aspecto sometidos a contracción térmica debido a gradientes de temperatura.

En el capítulo 4 se presentan los resultados experimentales obtenidos, tanto de la caracterización del material como de los ensayos de validación para el algoritmo desarrollado. En el capítulo 5 se muestran los resultados obtenidos a partir de la aplicación del algoritmo desarrollado y la validación de los mismos mediante resultados presentados por otros autores y los ensayos de laboratorio realizados. Finalmente, en el capítulo 6, se indican las principales conclusiones alcanzadas en la presente Tesis y se plantean algunas líneas de trabajo futuro.

\subsection{Aportes originales de la Tesis}

Como resultado de los trabajos realizados en la presente Tesis doctoral se han logrado los siguientes aportes originales:

- Se ha desarrollado un algoritmo que permite incorporar las propiedades mecánicas del hormigón de acuerdo con su distribución probabilística en un código de elementos finitos, considerando su evolución en el tiempo.

- El algoritmo ha sido desarrollado para asignar las propiedades mecánicas al elemento estructural analizado en forma aleatoria, lo cual representa lo que sucede en elementos estructurales reales. Para un mismo problema esta asignación aleatoria da lugar a distintos resultados, lo que permite realizar un análisis estadístico con los resultados numéricos obtenidos. 
- A partir de esta asignación aleatoria se propone un criterio basado en la heterogeneidad presente en el hormigón, para determinar zonas de fisuración preferentes y tiempos de aparición de fisuras, en estructuras de hormigón inicialmente intactas y sometidas a un estado tensional uniforme, como es el caso del desarrollado por fenómenos de contracción.

- Los resultados obtenidos mediante la aplicación del algoritmo desarrollado se validaron éxitosamente contrastándolos con ensayos de laboratorio y resultados de otros autores. 



\section{Capítulo 2}

\section{Antecedentes}

\subsection{Introducción}

El hormigón posee una distribución heterogénea de muchos componentes sólidos y de poros de varias formas y tamaños, llenos total o parcialmente con soluciones alcalinas, lo que le confiere una estructura altamente compleja. Además, las propiedades mecánicas del hormigón evolucionan en el tiempo, ya que dos de los componentes de su estructura, la pasta de cemento y la interfase entre el agregado y dicha pasta, también lo hacen.

Otro aspecto importante en el comportamiento del hormigón lo constituyen los cambios volumétricos a los que se encuentra sometido debido a distintas razones, en particular, al calentamiento y posterior enfriamiento luego del proceso de hidratación del cemento, la pérdida de humedad durante el secado, las deformaciones por cargas sostenidas en el tiempo y los ataques químicos. Estos cambios de volumen son de interés, ya que si se encuentran impedidos o limitados debido a las condiciones constructivas de la estructura pueden generarse tensiones que en el caso de superar la resistencia a tracción del material, provocan la aparición de fisuras.

En este capítulo se realizará una revisión bibliográfica de diferentes tipos de cambios de volumen que se presentan en el hormigón. En particular se describirá el fenómeno de contracción, dada la importancia del mismo como causa de fisuración en el hormigón. Posteriormente se describirán distintos métodos para la modelación del hormigón, haciendo énfasis en la representación numérica del material; para luego presentar los modelos disponibles que permiten analizar el comportamiento del mismo bajo la acción de distintos tipos de solicitaciones mecánicas. 


\subsection{Fenómenos de cambio de volumen en el hormi- gón endurecido}

En el hormigón los cambios de volumen tienen lugar por distintas razones: expansiones y contracciones generadas por cambios de temperatura y humedad (contracción térmica y contracción por secado), efectos químicos como la contracción por carbonatación, ataque de sulfatos y reacciones expansivas álcali-agregado y el creep o deformación debida a cargas sostenidas en el tiempo, Kosmatka et al. (2002); ACI (2007). Si los cambios de volumen no son uniformes, se generan tensiones por restricciones internas denominadas tensiones auto-inducidas o tensiones por gradiente de superficie. Si el hormigón pudiera deformarse libremente frente a estos cambios de volumen, los mismos no presentarían importantes problemas para las estructuras.

A su vez los cambios de volumen en los elementos de hormigón se encuentran restringidos por las fundaciones, las armaduras de refuerzo y la superficies de contacto cuando se produce el hormigonado del elemento estructural en etapas. Los efectos de las tensiones debido a restricciones externas, denominados también tensiones por gradiente de masa, se suman a los originados por las restricciones internas, van Mier (1997); Klemczak y Knoppik-Wróbel (2011). Si los niveles de tensión desarrollados superan la resistencia a tracción del hormigón aparecerán fisuras, Kosmatka et al. (2002); ACI (2007); Flaga y Furtak (2009); Klemczak y Knoppik-Wróbel (2011).

En la Figura 2.1 se ejemplifican fisuras por restricciones externas (izquierda) y por restricciones internas (derecha).

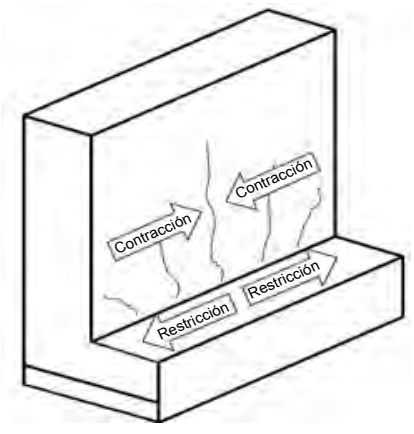

Fisuras por restricción externa

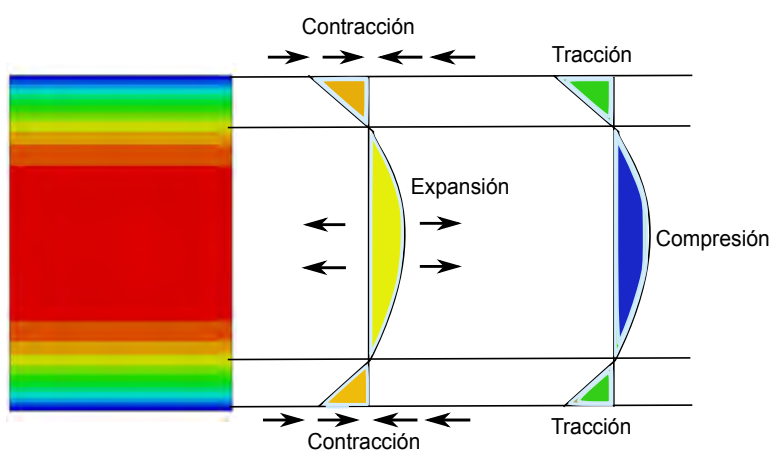

Fisuras por restricción interna

Figura 2.1: Fisuras por restricción externa, CIRIA-660 (2007) (izquierda), fisuras por restricción interna (derecha)

Las fisuras debido a restricciones internas son fisuras superficiales que, en general aparecen a edad temprana, antes que las fisuras por restricciones externas. La apertura de este tipo de fisuras disminuye con el tiempo. En cambio las fisuras por restricción externa en general aparecen a mayor edad 
y se desarrollan a lo largo de toda la sección del elemento estructural. La apertura de este tipo de fisuras aumenta con el tiempo.

Los factores que determinan las fisuras por restricciones internas o externas están controlados por la relación volumen/superficie $(V / S)$ de la estructura. En estructuras con relaciones $(V / S)$ pequeñas, por ejemplo tabiques, las fisuración por gradiente de superficie y gradiente de masa puede ocurrir en un corto período de tiempo, por lo que existe un acoplamiento entre los dos fenómenos, si bien los reglamentos ACI (2007); CIRIA-660 (2007) estudian estos fenómenos de manera desacoplada. En cambio en elementos con relaciones $(V / S)$ elevadas, por ejemplo plateas de fundación de grandes dimensiones o módulos de presas de hormigón, ambos fenómenos se desarrollan de manera desacoplada.

Las fisuras por gradiente de superficie son inductoras de las fisuras por gradiente de masa.

\subsubsection{Contracción}

La contracción en el hormigón puede definirse como un cambio de volumen que tiene lugar durante dos etapas consecutivas: contracción a edad temprana, la cual ocurre en las primeras 24 horas después del mezclado, y contracción a largo plazo, después de las 24 horas, Holt (2005); Esping (2007). Estas etapas dependen del nivel de humedad en el hormigón fresco y endurecido. De acuerdo a su origen, la contracción puede clasificarse en: contracción plástica, contracción química (la cual incluye la contracción autógena), contracción térmica y contracción por secado. Desde el punto de vista del largo plazo, también debe considerarse la contracción por carbonatación, Metha y Monteiro (1998); Kosmatka et al. (2002); Kucharczyková et al. (2017). En las etapas tempranas del fraguado y del endurecimiento la suma de los efectos provocados por todos estos tipos de contracción es significativa, sin embargo a largo plazo la contracción por secado parece ser la determinante, Neville (2011).

\subsubsection{Contracción plástica}

La contracción plástica puede tener lugar cuando el hormigón se encuentra todavía en estado plástico, desde la colocación hasta el fraguado final, Mindess et al. (2003); Kosmatka et al. (2002); Neville (2011). La contracción genera tensiones de tracción en la capa superficial ya que ésta se encuentra restringida por las capas interiores de hormigón. Debido a que el hormigón es poco resistente en estado plástico, aparecerán fisuras en la superficie, Neville (2011).

Una rápida pérdida de humedad generada principalmente por evaporación, ocasiona el secado de la superficie del hormigón y provoca que el mismo se contraiga fácilmente debido a la poca rigidez desarrollada hasta ese mo-

Ing. M. Paula Zappitelli 
mento, Mindess et al. (2003). Otro factor de importancia es la baja capacidad de deformación del hormigón durante el fraguado inicial, ya que ésta alcanza su valor mínimo.

La exudación puede compensar total o parcialmente el secado del hormigón, debido a que el agua asciende a la superficie por la incapacidad del sistema para retenerla. Sin embargo, si la velocidad de evaporación resulta mayor a la velocidad con la cual el agua es transportada hacia la superficie, se desarrollarán presiones capilares. Si las partículas superficiales no pueden permanecer cubiertas por una lámina de agua, se formarán meniscos entre estas partículas debido a las fuerzas de adhesión y tensión superficial. La tensión capilar actúa sobre estas partículas sólidas dando lugar a una contracción del material en estado plástico, los poros reducen su tamaño y adicionalmente se transporta agua hacia la superficie. Si el radio de los meniscos se vuelve demasiado pequeño para mantener unidas las partículas superficiales, la presión capilar se reduce permitiendo el ingreso de aire en el sistema de poros, comenzando por los poros más grandes, Schmidt y Slowik (2013). Esto provoca fuerzas de contracción entre las partículas debido a los gradientes de humedad, los cuales originan gradiente de deformaciones y en consecuencia tensiones por gradientes de superficie, que pueden dar lugar a la aparición de fisuras.

Finalmente luego del fraguado, el esqueleto sólido del hormigón es lo suficientemente rígido, por lo que la deformación vertical del hormigón se detiene completamente o continua en una muy baja proporción, Sayahi (2016).

En la Figura 2.2 puede observarse el mecanismo de la contracción plástica explicado anteriormente.

De acuerdo a lo explicado previamente, la contracción plástica será mayor cuanto mayor sea la velocidad de evaporación. Esto dependerá de la temperatura del aire, de la temperatura del hormigón, de la humedad relativa del aire y de la velocidad del viento. A su vez la contracción plástica también aumentará cuanto mayor sea el contenido de cemento en la mezcla o se reducirá cuanto mayor sea el contenido de agregados, Brooks (2015).

La contracción plástica puede controlarse minimizando la superficie de evaporación mediante el uso de membranas, protectores contra el viento, protectores contra la acción de sol, mantas pláticas, retardadores de evaporación y fibras plásticas, Kosmatka et al. (2002).

Este tipo de contracción es más predominante en superficies horizontales, como pavimentos y losas, en donde tiene lugar una rápida evaporación, Mindess et al. (2003).

\subsubsection{Contracción química}

Cuando el hormigón se encuentra en estado plástico, la contracción química consiste en la reducción del volumen absoluto de sólidos y líquidos en la 


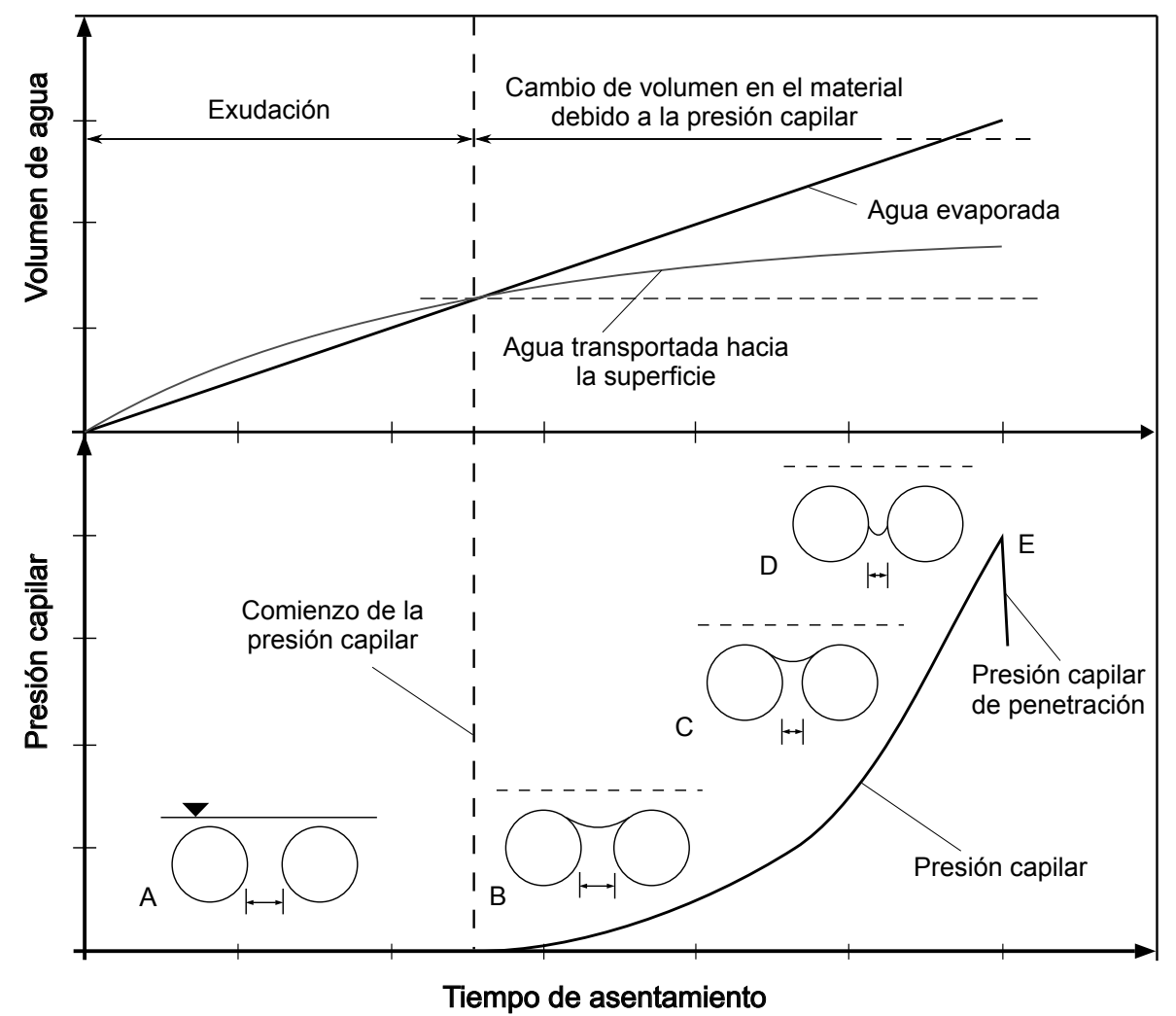

Figura 2.2: Mecanismo de la contracción plástica en el hormigón, Schmidt y Slowik (2013)

pasta como resultado de la hidratación del cemento. El volumen absoluto de los productos de hidratación del cemento es menor que el volumen absoluto de cemento y agua antes de la hidratación. Cuando el hormigón comienza a adquirir rigidez, la contracción química tiende a crear poros dentro de la estructura de la pasta, Kosmatka et al. (2002); Brooks (2015). En su mayor parte este cambio de volumen es interno, por lo que las dimensiones del elemento de hormigón no cambian visiblemente. En la Figura 2.3 se observan las razones por las cuales se originan los cambios de volumen debidos a contracción química y autógena en la pasta de cemento fresca y endurecida. La relación agua/cemento (a/c) y la finura del cemento afectan la tasa a la que ocurre la contracción autógena. La magnitud final de la contracción química cuando el grado de hidratación alcanza el $100 \%$ se encontrará influenciada solamente por la composición química del cemento, Holt (2001).

Según Power (1935) a los 28 días el aluminato tricálcico, $C_{3} A$, es el producto de hidratación que más se contrae y el silicato dicálcico, $C_{2} S$, es el que menos se contrae. La contracción que experimentan el silicato tricálcico, $C_{3} S$, y el 
ferroaluminato tetracálcico, $C_{4} A F$, son similares a la que presenta el $C_{2} S$.

\subsubsection{Contracción autógena}

La contracción autógena es una reducción macroscópica del volumen de la pasta de cemento, mortero u hormigón causada por la hidratación del cemento después del fraguado inicial, Tazawa (1999); Kosmatka et al. (2002); Mindess et al. (2003). Este tipo de contracción no considera los cambios de volumen debidos a la pérdida o ingreso de sustancias, variaciones de temperatura, aplicación de fuerzas externas o restricciones, por lo que constituye una reducción del volumen en el hormigón sin intercambio de humedad con el ambiente, Holt (2001).

Esta reducción de volumen es mucho menor que la reducción del volumen absoluto causada por la contracción química, debido a la rigidez de la pasta de cemento endurecida. La contracción química es la fuerza impulsora detrás de la contracción autógena, Kosmatka et al. (2002). Mientras la contracción química consiste en una reducción interna de volumen, la contracción autógena es un cambio de volumen externo, Holt (2001). En la Figura 2.3 se observa la relación entre la contracción química y la contracción autógena.

La magnitud y mecanismo de la contracción autógena dependen fuertemente de las proporciones de la mezcla y no de factores como los métodos de moldeo, colocación y curado del hormigón, Holt (2001).

En las etapas tempranas de la hidratación del cemento, cuando la mezcla aún se encuentra en estado plástico, la contracción química y la contracción autógena constituyen un mismo fenómeno por lo que generan en conjunto una reducción de volumen, como se observa en la Figura 2.3. Sin embargo, a medida que progresa la hidratación comienza a formarse el esqueleto del hormigón, por lo que éste puede resistir las tensiones inducidas por la contracción química. En este punto comienzan a diferenciarse la contracción autógena de la contracción química, Lura et al. (2003), debido a que el proceso de hidratación dará lugar a la formación de poros en la estructura del hormigón. La contracción autógena se relaciona con la resistencia y rigidez del esqueleto de hormigón, Tazawa (1999).

Una vez formado el esqueleto, la contracción autógena pasa a convertirse en auto desecación. A medida que la hidratación del cemento avanza, el agua libre es consumida gradualmente formándose poros finos. Para hormigones con baja relación a/c hay poca agua libre disponible para el proceso de hidratación. En busca de agua extra, el cemento la tomará primero de los poros capilares y luego del gel de cemento, con el objetivo de que siga progresando el proceso de hidratación. El consumo del agua de los capilares y del gel reduce la humedad relativa de los poros. Este fenómeno se conoce como auto desecación, debido a la reducción de la humedad interna sin transferencia de masa hacia el exterior, Copeland y Bragg (1955); Tazawa (1999). La auto desecación induce presiones negativas en los meniscos formados generando 
la contracción del hormigón.

La contracción autógena aumenta con la reducción de la relación a/c y con el incremento de la cantidad de pasta. En el caso de hormigones de cemento portlad normal la contracción autógena es despreciable. Sin embargo, es importante en hormigones con relaciones a/c menores a 0.42 , o con adiciones minerales con bajo peso específico en comparación con hormigones de cemento portlad normal, por ejemplo escoria de alto horno granulada fina y silica fume, Tazawa (1999); Holt (2001); Mindess et al. (2003).

Los hormigones susceptibles de experimentar valores considerables de contracción autógena deben curarse con agua externa durante 7 días para ayudar al control de fisuración. Inmediatamente después del moldeo deben emplearse membranas. La hidratación de materiales cementicios suplementarios también contribuye a la contracción autógena, aunque a un nivel diferente en relación a la generada por la pasta de cemento. Además de ajustar el contenido de pasta y la relación a/c, la contracción autógena puede limitarse mediante el empleo de aditivos para reducción de la contracción o de técnicas de curado interno, Kosmatka et al. (2002).

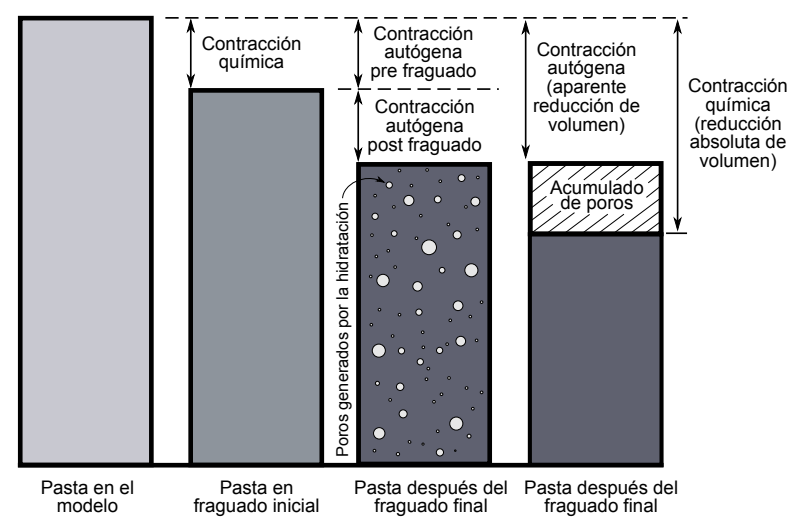

Figura 2.3: Cambios de volumen debidos a contracción química y autógena en la pasta de cemento fresca y endurecida, Kosmatka et al. (2002)

\subsubsection{Contracción por secado}

La contracción por secado representa la mayor proporción de los cambios de volumen que ocurren en el hormigón. La magnitud de la contracción por secado depende fuertemente de la cantidad de humedad que pierde el hormigón por el secado, lo cual es controlado por la humedad relativa del ambiente, Aly y Sanjayan (2009). El mecanismo de la contracción por secado es similar al mecanismo de la contracción autógena ya que las mismas ocurren 
debido a la pérdida de agua en el sistema de poros. Sin embargo, en la contracción por secado el agua es transferida hacia el exterior, mientras que en la contracción autógena el agua es transferida dentro de la estructura de poros, Khairallah (2009).

Cuando el hormigón se encuentra en contacto con el ambiente y en condiciones de baja humedad y/o alta temperatura, el agua comienza a evaporarse desde la superficie expuesta. Durante las primeras etapas de la contracción por secado existe agua libre que se mueve desde la masa del hormigón hacia la superficie debido al fenómeno de exudación, Holt (2001). Cuando el agua libre se evapora el proceso de secado continúa extrayendo el agua del gel desde el interior de la masa de hormigón, luego del secado de los poros más grandes. La evaporación del agua causa una reducción de la humedad relativa en los poros, la cual produce tensiones de tracción. Estas tensiones de tracción se equilibran con fuerzas de compresión en los alrededores del hormigón. Dichas fuerzas de compresión dan lugar a la contracción por secado, Khairallah (2009).

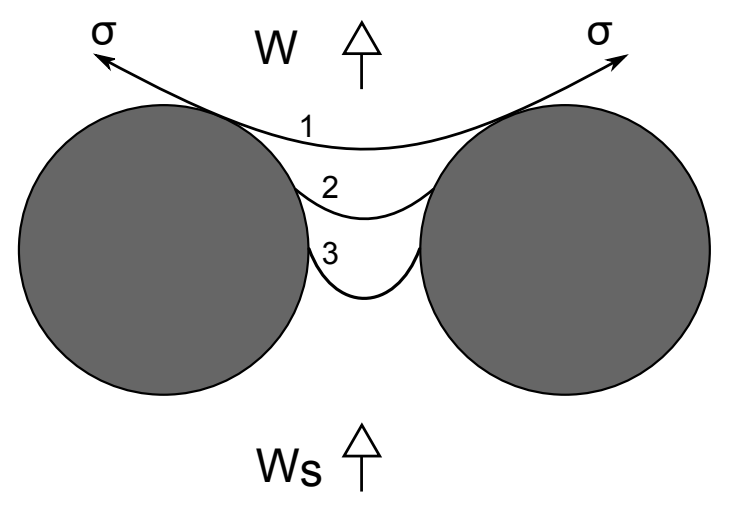

Figura 2.4: Mecanismo de la contracción por secado, Radocea (1992)

A medida que la evaporación del agua continúa, la tensión superficial responsable de la contracción por secado aumenta, como se observa en la Figura 2.4, Radocea (1992). La respuesta de la pasta de cemento a la pérdida de humedad depende de la presencia de agregados y de la forma del elemento estructural. Los factores que afectan a la contracción por secado pueden observarse en la Figura 2.5, Mindess et al. (2003).

Existen tres fenómenos que contribuyen a la contracción por secado de la pasta de cemento: la tensión capilar, las presiones de separación y los cambios en la energía superficial libre. Esto es resultado de la naturaleza de la pasta de cemento hidratada, su elevada porosidad con una red de pequeños poros 


\section{Parámetos de la pasta \\ Porosidad \\ Edad de la pasta Relación a/c y grado de hidratación \\ Temperatura de curado \\ Composición del cemento \\ Contenido de humedad \\ Adiciones}

\section{Parámetros del hormigón \\ Rigidez del agregado \\ Contenido de agregado (contenido de cemento) \\ Relación superficie/volumen \\ Espesor}

\section{Parámetros ambientales \\ Humedad relativa \\ Tasa de secado \\ Tiempo de secado}

Figura 2.5: Factores que afectan la contracción por secado, Mindess et al. (2003)

capilares, los extensos enlaces de van der Waals, la microporosidad intrínseca del S-C-H y la elevada área superficial, Mindess et al. (2003). A continuación se describirán cada uno de los fenómenos mencionados anteriormente.

- Tensión capilar: El agua que se encuentra en los poros capilares pequeños está parcialmente sometida a la influencia de las interacciones superficiales ejercidas por las paredes de los poros. Este agua no puede ser removida por evaporación a menos que la humedad relativa (HR) sea menor que un determinado valor. Este valor depende del radio del poro, y viene dado por la siguiente expresión:

$$
\ln (H R)=K\left(\frac{2 \gamma}{r}\right)
$$

en donde $r$ es el radio del poro, $\gamma$ la energía superficial libre del agua y $K$ una constante. El agua solo puede eliminarse a través de la creación de una interfase entre el agua y el aire, denominada menisco. La generación de la mencionada interfase requiere energía. Como resultado de la interacción del menisco con las paredes de los poros, éste adopta la forma de una superficie curva, y el agua pasa a estar en un estado de tensión hidrostática $\left(P_{\text {cap }}\right)$, en donde:

$$
P_{\text {cap }}=\frac{2 \gamma}{r}=\frac{\ln (H R)}{K}
$$

El agua ejerce una compresión hidrostática sobre el esqueleto sólido, 
y si se produce un reacomodamiento interno de las partículas algunos poros pueden reducir su tamaño.

Los poros capilares grandes, que se encuentran vacíos para HR inferiores a $95 \%$, desarrollan tensiones bajas debido a su alta relación área/volumen. Sin embargo, a humedades más bajas, aunque el volumen capilar se reduce las tensiones crecen rápidamente. Las tensiones capilares no pueden desarrollarse por debajo de una humedad relativa del $45 \%$, ya que los meniscos dejan de ser estables, Mindess et al. (2003).

- Presiones de separación: El agua es adsorbida en la superficie del S-C-H para cualquier nivel de HR. El incremento del espesor de la capa de agua es directamente proporcional al incremento de la humedad. El ensamblaje de partículas coloidales, como las del S-C-H, tiene lugar a través de fuerzas de van de Waals, las cuales atraen las partículas adyacentes y mantienen sus superficies en contacto. La adsorción de agua entre las superficies del S-C-H crea presiones de separación, las cuales resultan de la orientación de las moléculas de agua en la capa adsorbida. La tensión de separación se incrementa con el incremento del espesor del agua adsorbida entre partículas hasta que se superan las fuerzas de atracción de van der Waals, y las partículas se separan generando una dilatación. El S-C-H se forma en el estado de dilatación durante la hidratación y los espacios llenos de agua constituyen los microporos. Por lo tanto, en el primer secado (el que ocurre antes de que el hormigón vuelva a estar en contacto con agua) la disminución de las tensiones de separación, acompañadas de una baja HR, provocan que las partículas se mantengan juntas a través de fuerzas de van der Waals, y se produzca una disminución de volumen. Las presiones de separación son significativas solamente a HR inferiores a $45 \%$, Mindess et al. (2003).

- Energía superficial libre: Por debajo de HR del 45\%, en donde las tensiones capilares y las presiones de separación dejan de ser significativas, la contracción tiene lugar debido a cambios en la energía superficial. Cuando se remueve el agua más fuertemente adsorbida, la energía superficial libre del sólido comienza a incrementarse significativamente. Se sabe que una gota de agua se encuentra bajo presión hidrostática por su tensión superficial o energía superficial libre. Esta energía se representa mediante la ecuación, 2.3 ya que la gota se encuentra limitada por un menisco, Mindess et al. (2003):

$$
P_{s f e}=\frac{2 \gamma S}{3}
$$

donde $P_{s f e}$ es la presión media a la que se encuentra sometida una partícula sólida, $\gamma$ es la energía superficial y $S$ es la superficie específica 
del sólido. Ya que $S$ es grande en el caso del S-C-H, $P_{s f e}$ será grande y causará compresión en el sólido, Mindess et al. (2003).

Una fracción de la contracción por secado es irreversible. Para un hormigón convencional la contracción reversible se encuentra entre el $40 \%$ y el $70 \%$ de la contracción por secado, dependiendo a la edad a la cual ocurrió el primer secado, Brooks (2015). Esta irreversibilidad está fuertemente afectada por la historia de secado de la pasta de cemento. Si la pasta se seca lentamente, exponiéndola progresivamente a bajas humedades relativas, la contracción total es menor a la obtenida si la pasta se seca directamente a la menor humedad relativa. Esto es muy importante en el caso de secciones gruesas de hormigón, ya que el proceso de secado puede ser relativamente lento en este tipo de secciones, en donde la difusión del agua desde el hormigón determina la tasa de pérdida de humedad. A su vez, el tiempo en el cual la pasta se encuentra a una baja humedad relativa, también influye en la contracción irreversible, Mindess et al. (2003). Además, si el secado es acompañado de hidratación y carbonatación, aparecerán enlaces adicionales, siendo la porosidad de la pasta de cemento endurecida menor y su resistencia mayor, impidiendo el ingreso de agua, y dando así lugar a una mayor contracción por secado irreversible, Neville (2011).

En la Figura 2.6 se observa la reversibilidad e irreversibilidad de la contracción en el hormigón sumergido en agua y luego expuesto al aire comparada con la expansión sufrida por un hormigón continuamente sumergido en agua.

Otros factores que tienen influencia en la contracción por secado son los agregados y la forma de la estructura analizada.

En cuanto a los agregados, la contracción por secado en el hormigón es menor que la contracción por secado en la pasta de cemento debido a la restricción impuesta por el agregado, Mindess et al. (2003). Los agregados son dimensionalmente estables ante cambios de humedad y la restricción impuesta por los mismos depende de la cantidad de agregados presentes en el hormigón, su rigidez y del tamaño máximo del agregado grueso. A mayor tamaño máximo, mayores tensiones debidas a contracción por secado en la interfase agregado-pasta, lo cual incrementa la cantidad de fisuras en dicha interfase, Mindess et al. (2003).

Con respecto a la forma de la estructura, esto determinará la tasa de pérdida de humedad, y por lo tanto la tasa y magnitud de la contracción por secado.

\subsubsection{Contracción térmica}

La contracción térmica representa el cambio de volumen debido a la diferencia entre el pico de temperatura que alcanza el hormigón durante la hidratación a edad temprana (luego de la primera semana después de la co-

Ing. M. Paula Zappitelli 


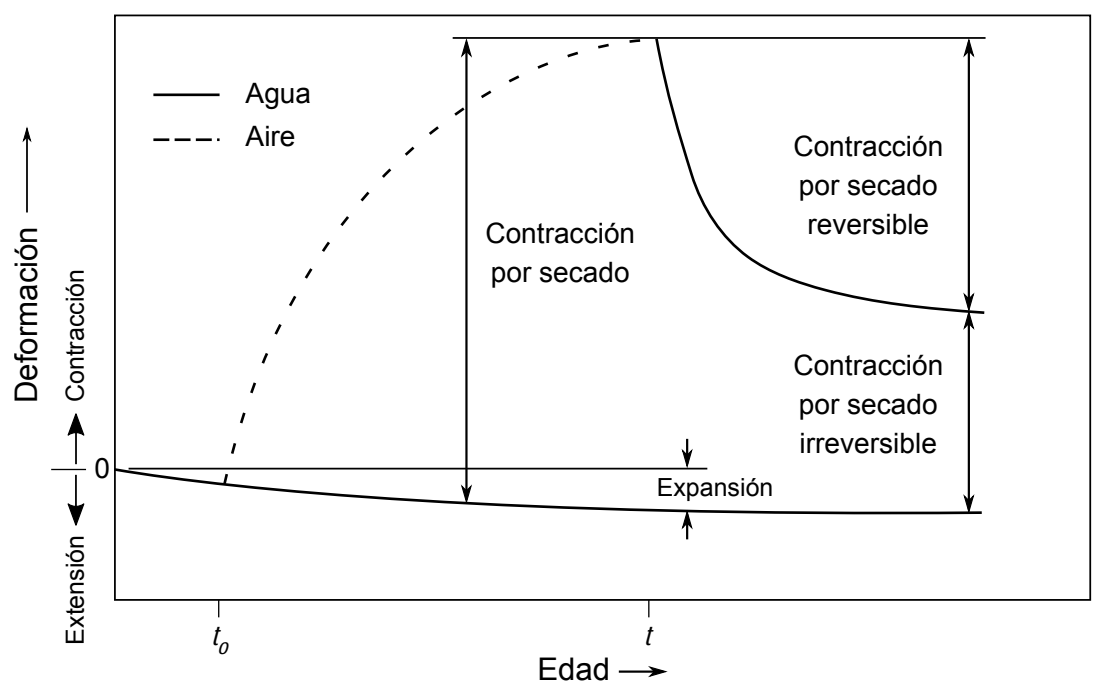

Figura 2.6: Reversibilidad e irreversibilidad de la contracción en el hormigón sumergido en agua y luego expuesto al aire comparada con la expansión sufrida por un hormigón continuamente sumergido en agua, Neville (2011)

locación) y la mínima temperatura a la que la estructura estará sometida durante las condiciones de servicio, ACI (2007).

A edad temprana la temperatura en el hormigón cambia debido al calor de hidratación del cemento. La fase de calentamiento depende de distintos factores, como la relación volumen/superficie, el contenido de cemento de la mezcla, la temperatura de colocación del hormigón y los procesos de intercambio de calor con el ambiente (convección, radiación). Por ejemplo en el caso de tabiques de hormigón la fase de calentamiento tiene lugar durante las primeras 12 horas, en cambio en estructuras de mayor envergadura, como presas puede durar mucho más tiempo. Luego el hormigón se enfría al estar en contacto con la temperatura ambiente. El gradiente resultante debido a la diferencia de temperatura generará deformaciones térmicas, Holt (2005). Estas deformaciones dan lugar a tensiones que pueden provocar la fisuración de la estructura si superan la resistencia a tracción del hormigón. Esto resulta de interés en particular en las estructuras de hormigón masivo, aquellas cuya menor dimensión es mayor a $1 \mathrm{~m}$, ya que en estos casos se tarda mucho más tiempo en alcanzar la temperatura de equilibrio, ACI (2007); Holt (2005). En la obras de hormigón masivo la principal preocupación es que la estructura permanezca monolítica, por lo que se busca controlar el incremento de temperatura mediante un adecuado diseño de las mezclas y la selección de las mejores condiciones de curado y del proceso constructivo, con el objetivo 
de evitar la aparición de fisuras.

En el caso del hormigón la deformación por contracción térmica es más importante que la expansión generada por el calor de hidratación del cemento, debido a que, dependiendo del módulo de elasticidad, el grado de restricción y la relajación debida al creep, los esfuerzos de tracción pueden ser lo suficientemente grandes como para originar la aparición de fisuras.

Los factores que afectan la contracción térmica son los siguientes, Metha y Monteiro (1998):

- Grado de restricción: Si un elemento de hormigón tiene libertad de movimiento no se desarrollará contracción térmica. Sin embargo, generalmente las estructuras de hormigón se encuentran restringidas externamente por la fundación e internamente por las deformaciones diferenciales que tienen lugar dentro del hormigón debido a la diferencia de temperatura entre diferentes áreas.

- Cambio de temperatura: Durante la hidratación del cemento tienen lugar reacciones exotérmicas que generan calor y el incremento de temperatura de la masa de hormigón. Este calentamiento causa una expansión, y la expansión restringida origina un esfuerzo de compresión. A edades tempranas, este esfuerzo será muy pequeño porque el módulo de elasticidad es bajo y la relajación del esfuerzo es alta. El cambio de temperatura se genera por la diferencia entre la temperatura de colocación del hormigón fresco, el incremento adiabático de la temperatura, la temperatura ambiente y la reducción de la temperatura debido a las pérdidas de calor. Para evitar la aparición de fisuras se controla la temperatura de colocación del hormigón mediante la incorporación de agregados enfriados y/o escamas de hielo con el objetivo de reducir la temperatura del hormigón en estado fresco.

- Elevación de la temperatura adiabática: La velocidad y magnitud de la elevación adiabática de la temperatura es función de la cantidad, composición y finura del cemento y de su temperatura durante la hidratación. Para reducir la elevación de temperatura adiabática se pueden emplear cementos de bajo calor de hidratación o incluir puzolana como un sustituto parcial del cemento.

- Pérdidas de calor: Dependen de las propiedades térmicas del hormigón (calor específico, conductividad térmica, coeficiente de expansión térmica) y de la metodología constructiva adoptada. La magnitud de la pérdida de calor a través de la superficie del hormigón es función del tipo del medio ambiente con el que se encuentra en contacto.

Ing. M. Paula Zappitelli 


\subsubsection{Contracción por carbonatación}

Este tipo de contracción ocurre como consecuencia de la reacción entre el hidróxido de calcio presente en el hormigón y el dióxido de carbono de la atmósfera en presencia de humedad. Debe mencionarse que la tasa de carbonatación es baja y su efecto se encuentra limitado a las capas superiores del hormigón, excepto en el caso de secciones delgadas. Esta contracción es más significativa cuando ocurre después del secado que durante el mismo, excepto para bajas humedades, Mindess et al. (2003). Sin embargo, la magnitud de la contracción por carbonatación es despreciable en comparación con las otras, Newman y Choo (2003).

Las reacciones que tienen lugar durante la carbonatación generan un reacomodamiento de la microestructura del hormigón, reducen la porosidad y provocan un cambio de volumen diferencial entre la superficie superior y la masa del hormigón, lo cual puede ocasionar el cuarteo de la superficie, Khairallah (2009). A su vez generan una reducción del ph del hormigón, dando lugar a la corrosión de las armaduras de acero, Brooks (2015).

Existen una serie de factores que afectan la contracción por carbonatación: permeabilidad del hormigón, contenido de humedad, humedad relativa, alta relación a/c, contenido de dióxido de carbono en el aire, Newman y Choo (2003). Para humedades altas la carbonatación es baja porque los poros están llenos de agua, por lo que el dióxido de carbono no puede penetrar en la pasta. Por otra parte, para humedades muy bajas, la ausencia de una película de agua reduce la tasa de carbonatación.

Cuando la contracción por carbonatación se suma a la contracción por secado, puede originarse fisuración severa por contracción.

\subsubsection{Expansión}

Este fenómeno se origina como resultado de la absorción de agua por parte del gel de cemento si existe un suministro continuo de agua durante el proceso de hidratación, Brooks (2015), y puede observarse durante las primeras 24 horas después del mezclado del cemento con el agua, Kucharczyková et al. (2017).

El gel de cemento conocido también como silicato de calcio hidratado, es una de la cuatro principales fases sólidas que componen la pasta de cemento hidratada, las otras tres son el hidróxido de calcio, los sulfoaluminatos de calcio y los granos de clinker no hidratados, Metha y Monteiro (1998).

Como consecuencia del agua absorbida por el gel de cemento tiene lugar la expansión, debido a que las moléculas de agua actúan en contra de las fuerzas de cohesión y obligan a las partículas del gel a separarse, originando una presión de expansión. Además el ingreso de agua proveniente del curado húmedo o por inmersión de la estructura, reduce la tensión superficial del gel provocando una expansión adicional, L' Hermite (1960); Kosmatka et al. 
(2002); Neville (2011).

También la expansión se vincula a la reabsorción del agua de exudación, la cual es expulsada de la pasta de cemento endurecida por la débil succión capilar luego de ocurrida la contracción plástica, Bentur (2003); Kucharczyková et al. (2017).

\subsubsection{Creep}

La deformación del hormigón bajo cargas se puede dividir en dos partes. Por un lado la deformación que ocurre en forma inmediata (deformación elástica), y por otro, la deformación dependiente del tiempo, que comienza en forma inmediata pero continúa aunque en menor proporción mientras el hormigón se encuentre bajo carga. Este último tipo de deformación se denomina creep.

La deformación por creep depende de la magnitud de la carga, la edad y resistencia del hormigón cuando se aplica la carga, el tiempo durante el cual el hormigón se encuentre bajo carga, características del agregado utilizado (tipo, cantidad y tamaño máximo), tipo de cemento, cantidad de pasta de cemento, relación volumen/área en el elemento de hormigón, cantidad de barras de refuerzo, condiciones de curado, temperatura ambiente y humedad, Kosmatka et al. (2002).

La deformación por creep puede dividirese en tres tipos: creep básico, creep por secado y creep térmico transitorio, Baẑant et al. (2004). El creep básico es altamente dependiente de la edad de carga y del contenido de agua, Baẑant et al. (1997). En cuanto a la edad de carga, ésta puede dividirse en dos etapas: edad química a corto plazo y edad no química a largo plazo. En la primera etapa los efectos del proceso de hidratación en la estructura de poros capilares de la pasta de cemento reducen el creep en el hormigón. En la segunda etapa el proceso de hidratación del cemento se encuentra prácticamente terminado, el creep básico se reduce mientras que la edad de carga aumenta, Baẑant et al. (2004, 1997).

El creep por secado tiene lugar cuando el hormigón se encuentra bajo carga durante y posteriormente al secado, Pickett (1942); Baẑant y Xi (1994). Existen dos tipos de mecanismos para este tipo de creep: un mecanismo macroscópico debido a microfisuaración y un mecanismo a nano escala debido a tensiones inducidas por contracción por pérdida de humedad y aumento de temperatura, Baẑant y Xi (1994). Según el mecanismo macroscópico, la distribución no uniforme de humedad entre las capas externas e internas del hormigón origina la contracción de dichas capas a diferente edad debido a la pérdida de agua de los poros capilares. En consecuencia, se inducen tensiones de tracción que provocan microfisuración en la superficie de la capa, originando deformaciones inelásticas no lineales en el hormigón (creep no reversible). En cuanto al mecanismo nanoscópico, al producirse el secado, 
el desequilibrio termodinámico entre el potencial químico de las distintas fases del agua de poros desarrolladas debido a la reducción de la presión de vapor en el poro y el aumento de temperatura, origina tensiones de tracción, las cuales se encuentran balanceadas por tensiones de compresión en los alrededores del hormigón, por lo que el mismo se contrae. Este fenómeno ocurre en particular en la nanoestructura del hormigón que comprende al silicato de calcio hidratado, los poros capilares muy pequeños y los poros del gel, Baẑant y Xi (1994).

El creep térmico transitorio se define como el incremento transitorio del creep debido a cambios de temperatura. Presenta un mecanismo macroscópico debido a una microfisuración inducida térmicamente, y un mecanismo a nano escala, en donde el cambio de temperatura altera el nivel de micro tensiones debido al cambio del potencial químico del agua de los nanoporos, Baẑant et al. (2004).

\subsubsection{Cambios de volumen debidos a efectos químicos}

Existen cambios de volumen provocados por reacciones químicas luego de la colocación del hormigón, o a largo plazo en el hormigón endurecido, ante la presencia de agua o humedad. Entre estos efectos pueden mencionarse el ataque por sulfatos y las reacciones que ocurren entre los álcalis del cemento y los agregados.

\subsubsection{Ataque por sulfatos}

Este fenómeno consiste en una expansión del hormigón por la formación de sólidos debido a la acción química o la cristalización de sales. Tiene lugar cuando el suelo y el agua subterránea presentan alto contenido de sulfatos y no se tomaron medidas para reducir los efectos del ataque, como por ejemplo, el uso de bajas relaciones a/c. En hormigones expuestos a humedecimiento y secado, como en el caso de las fundaciones, puede presentar efectos muy severos como extensas fisuras y desintegración del hormigón, Chaudhar y Chakrabart (2012).

\subsubsection{Reacciones álcali-agregado}

Algunos agregados reaccionan con los álcalis del cemento causando una expansión y provocando la fisuración del hormigón en estructuras ubicadas en ambientes húmedos como por ejemplo presas, pilas de puentes y muros marinos, Chaudhar y Chakrabart (2012).

Las materias primas utilizadas para la elaboración del cemento portland son las responsables de la presencia de los álcalis del cemento. Dependiendo del contenido de álcalis, el pH del fluido de poros en hormigones normalesvaría entre 12.5 y 13.5. Este $\mathrm{pH}$ representa un líquido cáustico o fuertemente 
alcalino en el que algunas rocas ácidas, como los compuestos de sílice y los minerales de sílice, no permanecen estables ante una larga exposición, Metha y Monteiro (1998). Ante el contacto con el agua, el gel de álcalis y silicatos tiende a expandirse. Si este gel se encuentra confinado por la pasta de cemento se generan presiones internas, las cuales provocarán la fisuración del hormigón, Brooks (2015).

Otro tipo de reacción dañina es la reacción álcali-carbonato, la cual ocurre por la reacción de dolomitas o calizas con los álcalis del cemento, Ba et al. (2008); Brooks (2015).

Para evitar este tipo de reacciones es importante el conocimiento de los agregados de la zona en la cual se realizará la estructura.

\subsection{Modelación numérica del comportamiento me- cánico del hormigón}

El hormigón es un material heterogéneo cuya estructura varía de acuerdo a la escala en la cual se lo analiza. Típicamente se utilizan tres escalas de análisis: micro escala, meso escala y macro escala, ver Figura 2.7, van Mier (1997).

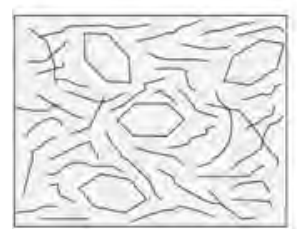

$10^{-6} \mathrm{~m}$

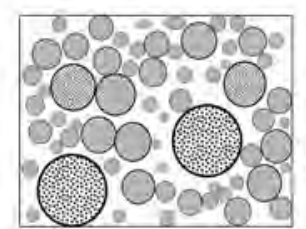

$10^{-3} \mathrm{~m}$

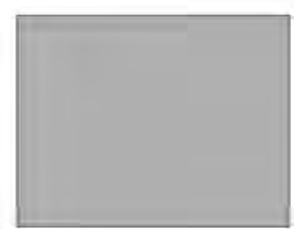

$10^{1} \mathrm{~m}$

Figura 2.7: Escalas de análisis en el hormigón, micro escala (izquierda), meso escala (centro), macro escala (derecha), van Mier (1997)

En la micro-escala, $\left(10^{-6} \mathrm{~m}\right)$, se puede observar la estructura interna del cemento y de la pasta de cemento endurecida (silicato de calcio hidratado, hidróxido de calcio). En la meso-escala, $\left(10^{-3} \mathrm{~m}\right)$, se observa la estructura compuesta por las partículas de agregado, la pasta de cemento endurecida y las interfases. Finalmente en la macro-escala, $\left(10^{1} \mathrm{~m}\right)$, correspondiente a la escala de trabajo de los ingenieros, no se observa estructura interna, excepto en el caso del hormigón armado, en donde se considera la estructura conformada por las barras de acero, van Mier (1997).

Existen dos enfoques para tener en cuenta la heterogeneidad del hormigón en los modelos numéricos. El primero de ellos se denomina enfoque directo, Yang et al. (2009), y consiste en modelar todas las fases del hormigón a nivel de meso escala (matriz de cemento, agregados e interfases), asignando a cada una de ellas sus correspondientes propiedades mecánicas. El otro enfoque se 
denomina enfoque indirecto, Yang et al. (2009), y considera la variación en el dominio analizado de las propiedades mecánicas del material, tales como la resistencia a la tracción y compresión. Lo cual puede modelarse en el marco del Método de los Elementos Finitos Estocásticos (SFEM, por sus siglas en inglés), Stefanou (2009), o considerando otros métodos.

El SFEM se define como un método que tiene en cuenta las incertidumbres presentes en la geometría o el material de una estructura, así como en las cargas actuantes, combinado con la discretización del dominio analizado utilizando el Método de los Elementos Finitos (MEF), Der Kiureghian y Ke (1988). SFEM consiste, en primer lugar, en determinar las variables aleatorias y campos aleatorios ("random fields"), es decir, aquellas cuyo comportamiento sigue una ley de distribución probabilística, como por ejemplo la resistencia a tracción, la resistencia a compresión o el módulo de elasticidad del material y las determinísticas las cuales se incorporan al análisis como es usual al aplicar MEF.

Es necesario hacer una distinción entre las variables aleatorias y los random fields. Las primeras se utilizan en simulaciones probabilísticas en donde la propiedad no varía espacial o temporalmente en todo el modelo, mientras que las segundas se emplean cuando la propiedad considerada sí lo hace, Hariri-Ardebili et al. (2018). Existen diferentes tipos de random fields debido a la naturaleza de la incertidumbre dentro del ambiente en que se realiza el estudio estocástico, Vanmarcke (2010). Dependiendo de la ubicación del punto de observación, pueden clasificarse en las siguientes categorías, Der Kiureghian y Ke (1988):

- Serie aleatoria: Se realizan observaciones en puntos discretos a lo largo de un eje temporal.

- Proceso lattice: Se realizan observaciones en un arreglo espacial constituido por una grilla regular o irregular.

- Función aleatoria continua: Se realizan observaciones en todos los puntos a lo largo de un eje coordenado espacial y/o temporal.

- Partición aleatoria del espacio: Se observa la variable aleatoria discreta en cada punto del dominio.

- Proceso de puntos aleatorios: Los puntos se ubican de acuerdo a un arreglo aleatorio en el espacio.

Para la generación de los random fields existen distintas técnicas, las cuales pueden clasificarse en dos grupos, Van der Have (2015). El primer grupo refiere a las variables aleatorias espacialmente correlacionadas y un método de discretización. Por cada punto en el dominio de elementos finitos, se genera una variable aleatoria correlacionada con otros puntos en el dominio del random field. Los puntos que se evalúan pueden ser puntos de integración 
de elementos finitos, nodos o puntos representativos, por ejemplo el centroide de un elemento finito. Las técnicas de discetización se pueden dividir en tres categorías:

- Método de discretización de puntos en el que se obtiene un random field constante a trozos. Dentro de esta categoría se encuentran el método del punto medio y el método del punto de integración.

- Método de discretización de puntos en donde se obtienen random fields continuos. Dentro de esta categoría se encuentran el método de la función de forma y la estimación lineal óptima.

- Métodos de discratización promedio, incluyen el método espacial promedio y el método integral ponderado.

El segundo grupo se basa en los métodos de expansión de series en donde los random fields se representan mediante la suma de funciones multiplicadas por una variable aleatoria. Estas funciones continuas tienen que ser integradas para poder aplicarlas en una malla de elementos finitos. Dentro de este grupo se pueden mencionar los siguientes métodos: expansión de polinomios mediante teoría del caos, expansiones de Karhunen Loève, expansión en series ortogonales, expansión óptima de estimación lineal, método Nyströn y métodos basados en el método de Galerkin.

De esta forma a cada punto del dominio analizado se le asigna un valor distinto de la variable aleatoria considerada. Luego esta distribución espacial del material se superpone a la malla de elementos finitos convencional y se aplica el Método de Montecarlo para hallar el equilibrio del sistema. En cada aplicación del Método de Montecarlo se genera un nuevo random field, Der Kiureghian y Ke (1988); Mahadevan y Haldar (1991); Matthies et al. (1997); Arregui-Mena et al. (2016).

La aplicación del Método de Montecarlo requiere la realización de muchas simulaciones, lo cual lo vuelve ineficiente para el caso de simulaciones a gran escala, Hariri-Ardebili et al. (2018). Por este motivo existen otros métodos que permiten reducir el número de muestras y en consecuencia la variabilidad en las respuestas obtenidas. Entre estos métodos puede mencionarse muestreo de importancia, Harbitz (1986), método del hipercubo latino, McKay et al. (1979), simulaciones de subconjunto, Au y Beck (2001) y diseño de experimentos, Cavazzuti (2012).

Además existen otras técnicas a aplicar en el caso de problemas con alto costo computacional:

- Métodos de perturbación: En estos casos las funciones estocásticas, como la matriz de elementos finitos estocásticos o el vector carga, se expresan como la suma de componentes determinísticos y aleatorios, Benaroya y Rehak (1988). Pueden utilizarse las expansiones en 
series de Taylor, en donde los términos de orden superior mejoran la aproximación. Muchas ténicas dentro de esta categoría apuntan a calcular la media, la varianza y los coeficientes de correlación, Sudret y Der Kiureghian (2000).

- Método de los Elementos Finitos Estocásticos Espectral: En este caso los random fields se expresan en base a la expansión de polinomios mediante la teoría del caos o expansiones de Karhunen Loève, Ghanem y Spanos (2003).

- Método de los Elementos Finitos de Confiabilidad: En este caso la probabilidad de que ocurra una falla en el sistema se evalúa sujeta a una función de estado límite, Sudret y Der Kiureghian (2002).

Como ejemplos de la aplicación de los métodos directos puede mencionarse la modelación del hormigón a nivel de meso escala utilizando elementos en las interfases y dentro de los agregados siguiendo una ley de ablandamiento elasto-plástica. En este caso se estudia la influencia del mallado y de la resistencia a la tracción y la energía de fractura, Trawiński et al. (2018). Utilizando el mismo modelo mencionado anteriormente, López et al. (2007) estudiaron el comportamiento del hormigón en compresión uniaxial y compresión diametral. A su vez determinaron la influencia de la cohesión y del ángulo de fricción interna en los resultados de los ensayos de compresión y analizaron el proceso de fisuración en hormigones de alta resistencia.

En otras publicaciones se modela la matriz, los agregados y las interfases del hormigón considerando la variación aleatoria de las propiedades mecánicas de los mismos según una distribución de Weibull. Para el estudio de la fisuración se utiliza un modelo de la mecánica de daño del continuo y se determina que el grado de heterogeneidad controla no solo la resistencia, sino también la ductilidad y la estabilidad de la propagación de las fisuras en el modelo utilizado. Se concluye que el comportamiento no lineal en la relación tensión-deformación del material proviene de la heterogeneidad de la estructura interna a nivel meso-escala, más que de la no linealidad en la ley constitutiva especificada para los elementos macroscópicos. Esto contrasta con los modelos macroscópicos para el hormigón, en los cuales la heterogeneidad del material se ignora, pero se utilizan leyes no lineales complejas para reproducir el comportamiento no lineal macroscópico observado en laboratorio, Zhu et al. (2004). El modelo de mecánica de daño del continuo mencionado anteriormente, también se aplica al estudio de ensayos de tracción directa, compresión, compresión diametral, corte y en hormigones reforzados con fibras de carbono, Teng et al. (2004).

En otros casos la modelación numérica de la meso-escala del hormigón se realiza a partir de imágenes en rayos x, obteniendo así la ubicación y forma de agregados e interfases, Huang et al. (2016); Trawiński et al. (2018); Sencu et al. (2016); Tal y Fish (2018). 
También se ha desarrollado un tipo de elemento de meso-escala en el que se considera el aporte de la matriz y los agregados en la definición de las propiedes del material. En este caso no se tiene en cuanta la interfase, Du et al. (2012).

Por otro lado, se han realizado modelos a meso-escala empleando una ley de inicio de fisuración que combina el criterio de tensiones principales máximas con un criterio de la Mécanica de Fractura Elástica Lineal para la punta de la fisura, y se incorpora un algoritmo de cola para definir la propagación de la fisura, Kwana et al. (2017).

Otro de los estudios realizados consiste en considerar la influencia de la variación de la forma del agregado modelado (circular, elíptico, poligonal), en las propiedades macroscópicas del hormigón (curva tensión-deformación). Se realiza un análisis de sensibilidad de distintos parámetros: mallado y consideración o no de la interfase. En este caso el modelado de la celda unitaria de hormigón se realiza a partir de imágenes obtenidas por tomografía computada, Chen et al. (2018).

Finalmente, también se han utilizado modelos a meso-escala para predecir fisuración por contracción mediante un indicador de durabilidad, Tang et al. (2013a).

Como ejemplos de aplicación de métodos indirectos, en el marco de SFEM puede mencionarse la utilización de random fields, considerando una distribución de Weibull para la resistencia a tracción, con el objetivo de modelar fisura cohesiva y estudiar el proceso de fisuración en celdas unitarias en dos y tres dimensiones, Yang et al. (2009); Su et al. (2010).

Por otro lado se han utilizado ramdom fields para considerar estádisticamente el efecto tamaño, Bouhjitia et al. (2018) y para el estudio sísmico de presas de hormigón, Hariri-Ardebili et al. (2018); Tang et al. (2013b).

Los random fields también se han empleado para considerar la variación estádistica de las propiedades mecánicas del hormigón en combinación con un análisis secuencial lineal como alternativa a MEF, para solucionar los problemas de convergencia, Stefanou et al. (2014).

Finalmente como ejemplos de métodos indirectos fuera del marco de SFEM, puede mencionarse el estudio de la fisuración térmica simulando el comportamiento de sólidos frágiles (rocas) bajo la acción de un shock térmico mediante el Método de los Elementos Finitos (MEF), considerando una distribución de Weibull para la resistencia a la tracción y el módulo de elasticidad. Estas propiedades mecánicas se asignan utilizando números aleatorios a cada uno de los elementos en que se divide la malla de elementos finitos, Tang et al. (2016).

En otros casos se realiza un análisis aleatorio a meso-escala y a partir de los resultados obtenidos se pasa a un análisis homogéneo a macro escala, justificando esto en que el comportamiento en escalas menores afecta el comportamiento a macro-escala, Jehel (2016). 
Por otro lado, se ha propuesto un modelo de disipación local de energía, en donde los parámetros del mismo se determinan aleatoriamente, para predecir la aparición de macrofisuras, Tailhan et al. (2014).

También puede realizarse la modelación de la meso-escala a nivel de elemento finito, considerándola a través de los niveles tensionales que experimentan cada una de las fases componentes, Zhong et al. (2011).

Finalmente puede mencionarse que se ha utilizado una combinación de los enfoques directo e indirecto para el modelado de la fisuración en vigas de hormigón entalladas, Elías et al. (2015).

\subsubsection{Modelos para el estudio del comportamiento del hor- migón bajo carga}

Los distintos modelos que permiten estudiar rigurosamente el fenómeno de la aparición y propagación de fisuras pueden dividirse en dos grandes grupos a partir de la forma en que se representan las fisuras en el modelo numérico: representación geométrica y representación no geométrica, Ingraffea (2007).

En el primer grupo la fisura es una entidad geométrica, por lo que el modelo geométrico y el modelo discretizado se actualizan con el crecimiento de la misma.

En el segundo grupo el modelo geométrico no contiene la fisura y la discretización no cambia durante el crecimiento de la misma. En este caso la fisura se encuentra representada, ya sea en el modelo constitutivo o en un modelo cinemático, en base a las deformaciones.

En la Figura 2.8 se muestran las subcategorías en que se dividen los dos grandes grupos descriptos anteriormente.

Dentro de los métodos de representación geométrica, se incluirán la Mecánica de Fractura Elástica Lineal (MFEL) y el Modelo de Fisura Cohesiva (MFC). Estos modelos se encuentran entre los métodos de restricción de forma, en donde el crecimiento de la fisura está restringido por el método de discretización o por la forma de la fisura, Ingraffea (2007).

Dentro de los métodos de representación no geométrica en la presente tesis se describirán los modelos de plasticidad con daño y el Método de los Elementos Finitos Extendido (XFEM), correspondiente a los métodos cinemáticos. Los métodos constitutivos son aquellos en que la rigidez del material se degrada localmente para simular la discontinuidad en el desplazamiento originada por la fisura, mientras la geometría y la malla permanecen sin cambios. En el caso de los modelos cinemáticos, el efecto de la fisura en los campos de deformaciones o desplazamientos circundantes se incorpora dentro de los elementos, dejando también la geometría y la malla sin alteraciones, Ingraffea (2007). 


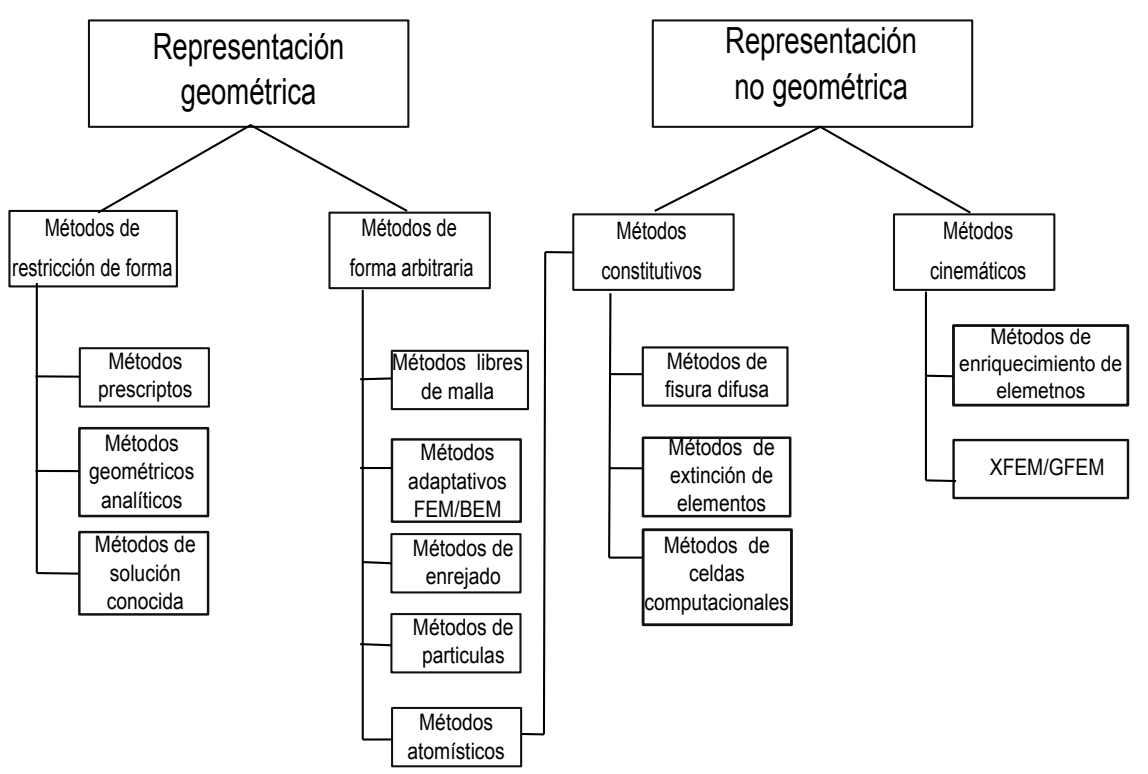

Figura 2.8: Clasificación de los métodos para representar fisuras en modelos numéricos, Ingraffea (2007)

\subsubsection{Mecánica de Fractura Elástica Lineal (MFEL)}

Los inicios de la Mecánica de Fractura se remontan a comienzos del siglo XX, cuando Inglis (1913) presenta el estudio del estado tensional de una placa infinita con un agujero elíptico cargada en sus extremos. La fisura constituía una singularidad en la placa, alterando el trayecto de las tensiones principales máximas. En la punta de la fisura tenía lugar una concentración de tensiones.

A partir de los resultados obtenidos por Inglis, Griffith notó que el criterio de resistencias no podía aplicarse en este tipo de problemas porque la tensión en la punta de la fisura es infinita, sin importar lo pequeña que sea la carga aplicada, Griffith $(1921,1924)$. Además concluyó que para la formación de una fisura se necesita cierta cantidad de energía por unidad de área en el plano de la misma, siempre que la estructura sea lo suficientemente larga, tal que la región de la punta de la fisura, en la que el proceso de fractura tiene lugar, sea despreciable. Esta energía es una propiedad del material y se denomina energía de fractura $\left(G_{f}\right)$. Sin embargo, existen aproximaciones más generales que consideran que la energía requerida para el crecimiento de una fisura depende de la historia de fisuración en lugar de ser una constante. En estos casos la energía requerida para un avance unitario de la fisura se denomina resistencia al crecimiento de la fisura $(R)$. Cuando la zona inelástica es despreciable puede considerarse que $R=G_{f}$, Baẑant y Planas (1997). Si la energía disponible es menor que la requerida, la fisura no crecerá. Si 
la energía disponible es igual a la energía requerida, la fisura crecerá estáticamente, es decir, con fuerzas de inercia despreciables. Pero si la energía disponible excede la requerida, la fisura crecerá de forma inestable, Baẑant y Planas (1997).

El planteo realizado por Griffith en términos energéticos se conoce como planteo global de la fractura, Elices Calafat (1998).

Años más tarde Irwing (1957) reformuló el problema de la Mecánica de Fractura Elástica Lineal en términos de los estados tensionales en el material cercano a la punta de la fisura, en lugar de realizar un planteo energético. Esto se conoce como planteo local de la fractura y es equivalente al planteo global de Griffith, Elices Calafat (1998). Cuando un cuerpo contiene una fisura, se desarrolla una fuerte concentración de tensiones alrededor de la punta de la misma. Si el material se comporta como lineal elástico e isótropo, excepto en una pequeña zona en proceso de fractura $(\mathrm{ZPF})$, la concentración de tensiones tiene la misma distribución cerca de la punta de la fisura cualquiera sea el tamaño, forma y condiciones de contorno del cuerpo. Sólo varía la intensidad de concentración de la tensión. Para la misma intensidad, las tensiones alrededor y cercanas a la punta de la fisura son idénticas, Irwing (1957). Los campos de tensiones y desplazamientos en el fondo de la fisura se caracterizan a través de un parámetro denominado factor de intensidad de tensiones $\mathrm{K}$, por lo que es posible expresar $G$ en función de $K$ siempre que se elija un camino de integración suficientemente cercano al fondo de la fisura, entonces el criterio local de fractura puede escribirse $K=K_{c}$, donde $K_{c}$ se denomina tenacidad de fractura y es una característica del material, Elices Calafat (1998)

Se denomina zona en proceso de fractura a una zona con comportamiento no lineal caracterizada por un ablandamiento progresivo, por el cual las tensiones decrecen a medida que aumentan las deformaciones. Ésta se encuentra rodeada por una zona sin ablandamiento caracterizada por endurecimiento o plasticidad perfecta, para la cual las tensiones crecen al incrementarse las deformaciones o permanecen constantes, Baẑant y Planas (1997).

Los modos de fractura pueden clasificarse en tres tipos: modo I en donde predominan las tensiones normales, modo II en donde predominan las tensiones de corte en el plano $\mathrm{y}$-x y modo III en donde predominan las tensiones de corte en el plano y-z, como se observa en la Figura 2.9, Kumar y Barai (2011).

Una de las principales razones por la cual los ingenieros emplean la mecánica de fractura es el denominado efecto tamaño. En las teorías clásicas basadas en plasticidad o análisis límites, la resistencia de estructuras geométricamente similares es independiente del tamaño de las mismas. En las estructuras de hormigón esto no ocurre, Baẑant y Planas (1997); Kumar y Barai (2011). Por este motivo la validez de la aplicación de la MFEL en estructuras de hormigón depende de la extensión de la zona microfisurada, del 

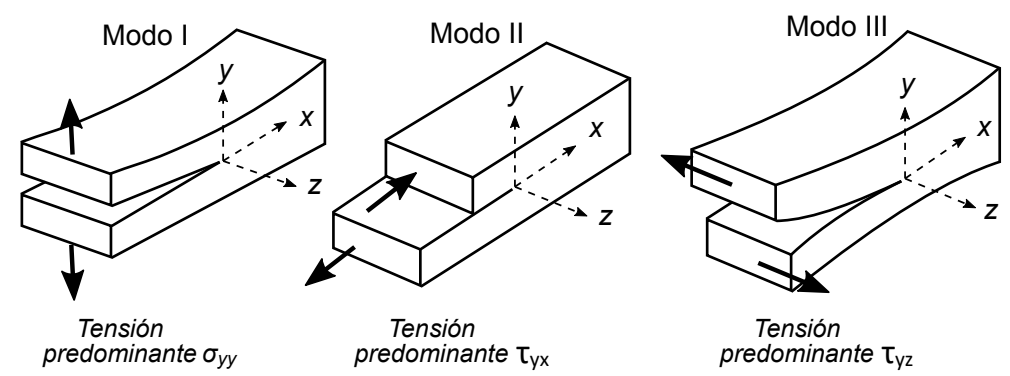

Figura 2.9: Modos de fractura, Kumar y Barai (2011)

lento crecimiento de la fisura o de otros comportamientos inelásticos cerca de la punta de la misma. Si el elemento analizado es lo suficientemente grande, la zona en la que las tensiones se encuentran perturbadas puede considerarse rodeada por un área en la que las tensiones concuerdan con la distribución de tensiones ideal y la MFEL puede aplicarse para determinar la carga de fisuración. De lo contrario, si el espécimen es pequeño en relación a la zona microfisurada, la MFEL no puede aplicarse como criterio de falla, Kumar y Barai (2011); Plizzari y Saouma (1995).

\subsubsection{Modelo de Fisura Cohesiva (MFC)}

Como se expuso en la sección anterior, la MFEL es aplicable únicamente cuando la zona en proceso de fractura es pequeña en comparación con la dimensión más relevante del espécimen en estudio.

Existen otros modelos que permiten describir el proceso de fractura en forma completa, lo cual resulta esencial cuando la longitud de la fisura u otra dimensión del elemento en estudio son pequeñas en relación con una zona en proceso de fractura completamente desarrollada. Esto ocurre en estructuras de hormigón o cerámicas, Baẑant y Planas (1997). El MFC considera la existencia de una fisura ficticia capaz de transmitir tensiones a través de sus caras. Este modelo fue originalmente desarrollado para representar diferentes procesos no lineales localizados en el frente de fisuras pre-existentes, siendo utilizado por los investigadores para distintos materiales, por ejemplo metales, polímeros, cerámicos y geomateriales, Dugdale (1960) y Barenblatt (1962).

Barenblatt (1962) introdujo una zona cohesiva para explicar el comportamiento no lineal de uniones atómicas rotas durante la propagación de fisuras. 
Su análisis mostró que la zona cohesiva permite revivir la singularidad de la punta de la fisura, mientras que para fisuras largas las ecuaciones de la MFEL se conservan. La energía de fractura, $G_{f}$, se relaciona con el potencial interatómico. Su análisis se limitó a fisuras muy largas comparadas con la zona cohesiva y considerando únicamente el estado crítico, es decir, el inicio del crecimiento estable de la fisura.

Simultáneamente, se propuso un modelo relativamente simple para tratar la plasticidad en la punta de la fisura, en el que se asumió que la tensión en la línea de la fisura más allá de la punta estaba limitada por la tensión de fluencia, y que la deformación plástica se concentraba a lo largo de la línea de la fisura, generando así una discontinuidad en el desplazamiento similar a una fisura, aunque se preserva la continuidad del material, Dugdale (1960). Esta aproximación puede justificarse en base al flujo de dislocaciones que se combinan y concentran en la línea de la fisura. En su origen, en el modelo anterior no se incluía ningún criterio de ablandamiento o de fractura, es un modelo puramente plástico.

El concepto de fisura cohesiva se aplicó al hormigón proponiendo que la misma se extiende en todo el material, incluso sin la presencia de una macrofisura, denominando a esta extensión del MFC modelo de fisura ficticia, Hillerborg et al. (1976). La formulación matemática de este modelo es la misma que la utilizada para las fisuras cohesivas clásicas. El modelo se basa fundamentalmente en dos cuestiones. Por un lado, en considerar que al someter a una probeta de hormigón a un estado de tracción uniaxial, luego de alcanzar la carga pico, todas las deformaciones se localizan en la fisura, que divide a la probeta en dos partes. Esto se basa en el hecho de que si se ensaya una probeta de hormigón a tracción sin llevar a cabo con cuidado especial el ensayo, se obtendrá una curva tensión-elongación aproximadamente lineal hasta la tensión pico y luego ocurrirá una rotura catastrófica a través de una única fisura, como se observa en la Figura 2.10 b. Esta fisura no es uniforme, debido a la heterogeneidad del hormigón y solo es perpendicular a la probeta en promedio. Sin embargo, esto puede ignorarse y aproximar la falla final a través de una fisura suavizada perpendicular al eje longitudinal de la probeta, como se observa en la Figura $2.10 \mathrm{c}$. Puede considerarse que la deformación permanente de cada una de las partes en las cuales se rompe la probeta es muy pequeña, a lo sumo del orden de la deformación inelástica medida justo antes del pico.

La segunda cuestión a tener en cuenta es la evolución de la fisura referida anteriormente, desde la probeta en estado intacto hasta su completa rotura, lo cual se justifica mediante la evidencia experimental, ya que que si la probeta traccionada es lo suficientemente corta y se utiliza una máquina de ensayos lo suficientemente rígida, la fisura puede evolucionar en forma estable, haciendo posible obtener la curva tensión-elongación en forma completa, incluyendo la región post-pico, como se observa en la Figura 2.10 d, 
(a)

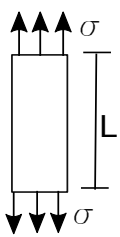

(b)

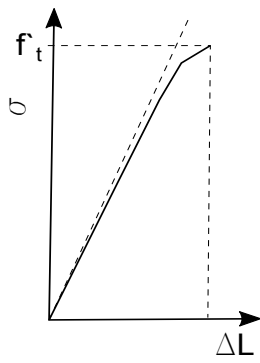

(c)

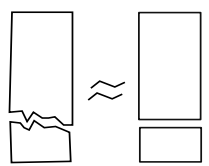

(d)

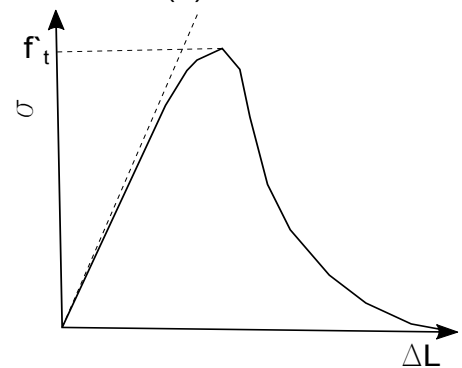

Figura 2.10: (a) Ensayo de tracción uniaxial en una probeta de hormigón. (b) Curva tensión-elongación con una sola fisura. (c) Fisura suavizada perpendicular al eje de la probeta (d) Curva tensión-elongación en un ensayo de tracción estable, Baẑant y Planas (1997)

Hughes y Chapman (1966), Evan y Marathe (1968). Esto demostró que la zona de transición desde la tensión máxima a la tensión cero es gradual y no instantánea. Además luego de la carga pico, las deformaciones se localizan en una región muy angosta, la cual se convertirá en una fisura visible, mientras que el resto de la probeta permanece descargada.

En base a lo anterior puede describirse el ensayo de tracción de una probeta de hormigón en forma idealizada, como se indica en la Figura 2.11. Hasta el pico, la carga se incrementa mientras las deformaciones se mantienen distribuidas uniformemente a lo largo de la probeta (arco $O-P$ ). Al alcanzar la carga pico aparece una fisura cohesiva normal al eje de la probeta en algún lugar de la misma, para desarrollar luego del pico una apertura finita, denominada $w$, mientras continúa transmitiendo tensiones, al mismo tiempo que el resto de la probeta se va descargando y su deformación decrece uniformemente a lo largo del arco $P$ - $B$. Por lo tanto la elongación total en el punto $A$ será la suma de una deformación uniforme correspondiente al punto $B$ y a la apertura de la fisura, como se indica en 2.4:

$$
\Delta L=L_{\varepsilon_{B}}+w
$$

en este caso $L$ representa la longitud de la probeta y $\varepsilon_{B}$ la deformación del material que no contiene a la fisura el cual se descarga desde el pico.

Luego se asumió que la tensión transferida a través de la fisura es una función de la apertura de la misma, definiéndola como se indica en 2.5, Hillerborg et al. (1976):

$$
\sigma=f(w)
$$

donde $f(w)$ es una función característica del material que debe determinarse experimentalmente, denominada función de ablandamiento, y corresponde al arco $P$ - $A$ de la Figura 2.11. Por definición la curva de ablandamiento verifica 
la propiedad de que la tensión límite para una apertura de fisura igual a cero será la resistencia a tracción.

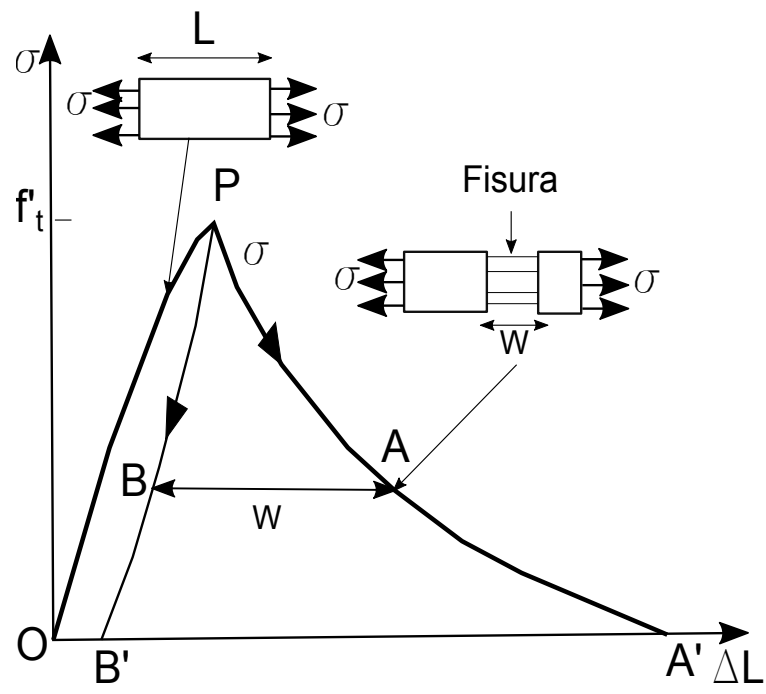

Figura 2.11: Ensayo de tracción idealizado de una probeta de hormigón, Baẑant y Planas (1997)

Para simplificar los cálculos, pero manteniendo la esencia del modelo, se asumió que la deformación inelástica en el camino carga-descarga es despreciable, es decir, el comportamiento del material fuera de la zona fisurada es elástico lineal, utilizando así una curva como la indicada en la Figura 2.12, Hillerborg et al. (1976). La elongación post-pico puede calcularse como se indica en 2.6:

$$
\Delta L=L \frac{\sigma}{E}+w=L \frac{f(w)}{E}+w
$$

donde $E$ es el módulo de elasticidad.

Por lo tanto, para describir la falla cuasi-estática de una probeta sometida a tracción se necesita conocer el comportamiento en tensión-deformación del material, incluyendo el comportamiento en carga y descarga, el criterio de fisuración, indicando la condición de aparición de la fisura cohesiva y su orientación y la ley de evolución de la fisura cohesiva, Elices y Planas (1989).

Todo lo expuesto anteriormente, correspondiente al caso de tracción pura, puede extenderse a otras situaciones para obtener un modelo más general, incluyendo el comportamiento no lineal del material, efecto de la triaxilidad en el criterio de fisuración y en la ley de evolución y el efecto del corte en los labios de la fisura, Elices y Planas (1989) y Planas et al. (1995a).

Es interesante señalar que si el material rompe completamente tal que la apertura de la fisura alcanza su valor crítico, $w_{c}$, el modelo de Dugdale (1960) es formalmente equivalente al modelo de fisura cohesiva, en el sentido 


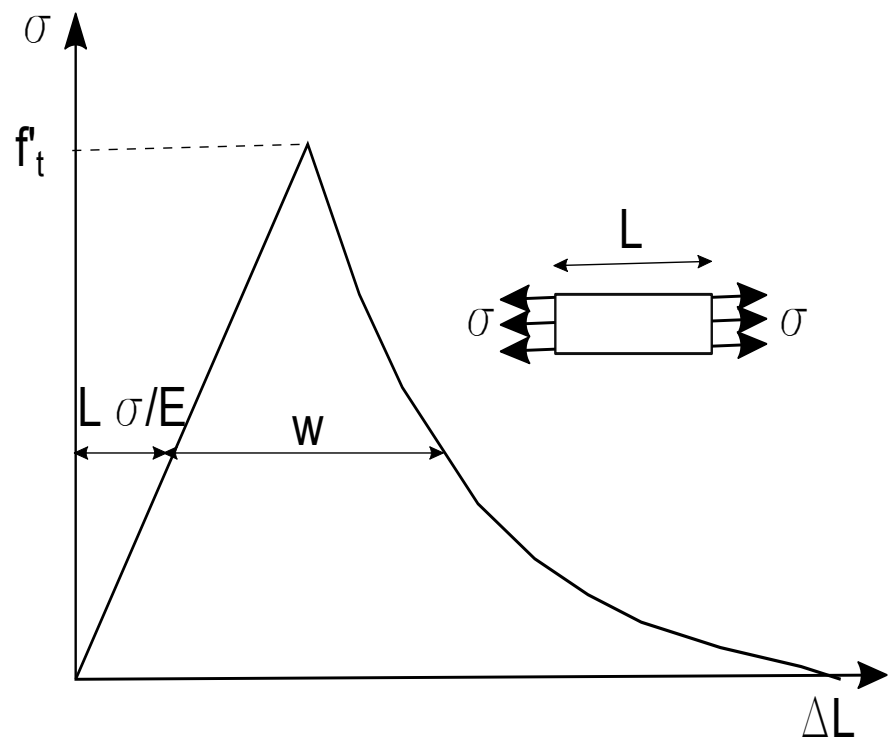

Figura 2.12: Curva de ablandamiento con comportamiento lineal hasta la carga pico, Baẑant y Planas (1997)

de Hillerborg et al. (1976), con una función de ablandamiento rectangular, según la cual el material cumple $\sigma=f(w)=\sigma_{y}=$ cte para $0<w<w_{c}$, y $\sigma=f(w)=0$ para $w>w_{c}$. En la Figura 2.13 se observa el modelo original de Dugdale (1960) junto con la función de ablandamiento rectangular. Esta función usualmente no es adecuada para obtener resultados precisos, sin embargo presenta la importante ventaja de poder ser utilizada para el estudio analítico de diferentes casos y captura correctamente la evolución principal del comportamiento en fractura, Baẑant y Planas (1997).

Es importante señalar que el parámetro principal del MFC es la curva de ablandamiento del material, de la cual se desprenden dos propiedades importantes: la resistencia a tracción, $f_{c t}$, y la energía de fractura, $G_{f}$ Baẑant y Planas (1997).

La resistencia a tracción es la tensión para la cual la fisura se origina y comienza a abrirse y la energía de fractura es la energía externa requerida para formar una superficie unitaria de fisura cohesiva.

La energía de fractura puede calcularse a partir de la siguiente integral:

$$
G_{f}=\int_{0}^{\infty} \sigma d w=\int_{0}^{\infty} f(w) d w
$$

Donde $\sigma$ es una tensión normal y $\omega$ representa la apertura de la fisura.

Geométricamente $G_{f}$ representa el área bajo la curva de ablandamiento, Baẑant y Planas (1997).

Un parámetro esencial que controla propiedades estructurales interesan-

Ing. M. Paula Zappitelli 


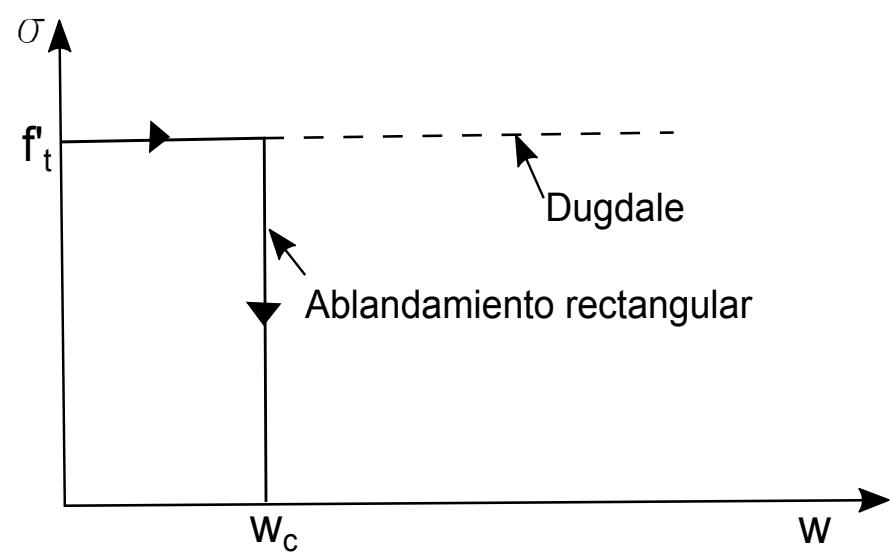

Figura 2.13: Tensión cohesiva vs apertura de fisura para el modelo original de Dugdale y para el ablandamiento rectangular, Baẑant y Planas (1997)

tes es la pendiente inicial de la curva de ablandamiento, Planas y Elices (1992), Baẑant y Li (1995), Planas et al. (1995b) y Planas et al. (1997). En situaciones en las que ningún punto de la estructura se encuentra bajo un gran ablandamiento puede utilizarse una curva de ablandamiento lineal. Esto es importante porque en probetas o estructuras entalladas de dimensiones no muy grandes o en estructuras sin entalla de cualquier tamaño, la carga pico ocurre antes de que se produzca un ablandamiento importante.

Debe señalarse que cada material presenta una curva de ablandamiento distinta, en particular, cada mezcla de hormigón originará una curva de ablandamiento diferente, la cual deberá obtenerse mediante un ensayo. Sin embargo se observó que al realizar un determinado número de ensayos la forma de la curva de ablandamiento es similar para diferentes mezclas de un hormigón ordinario, Petersson (1981). Si las curvas obtenidas a partir de los ensayos se adimensionalizan se observa que la forma obtenida es muy similar.

A pesar de la forma no lineal de las curvas de ablandamiento resultantes de los ensayos, es conveniente introducir expresiones analíticas simplificadas para aproximar estas curvas, con el objetivo de realizar análisis e inferir comportamientos para estructuras particulares, Baẑant y Planas (1997). Estas simplificaciones pueden observarse en la Figura 2.14.

La curva de ablandamiento rectangular captura la tendencia principal del proceso de fractura y el efecto tamaño, pero usualmente sobreestima la resistencia de probetas y estructuras de tamaño normal (profundidad del orden de decímetros).

Por otro lado, la curva de ablandamiento lineal resulta elegida cuando no se tiene demasiada información sobre el comportamiento del material. 


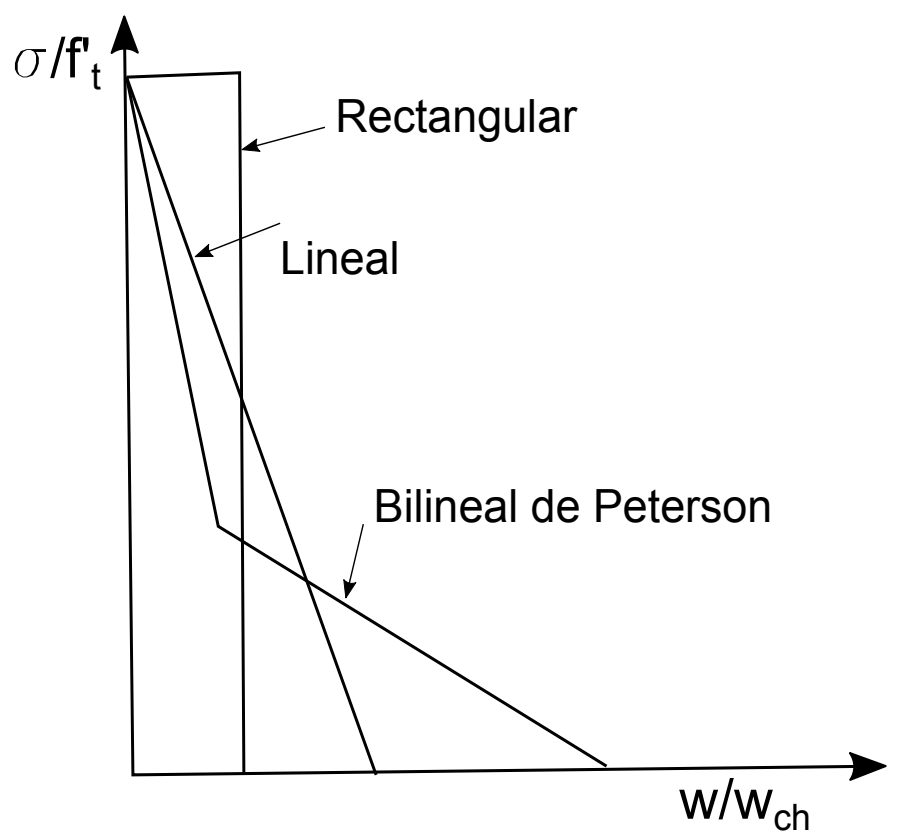

Figura 2.14: Curvas de ablandamiento rectangular, lineal y bilineal de Peterson, Baẑant y Planas (1997)

\subsubsection{Modelos de plasticidad con daño}

En la literatura existen distintos tipos de modelos constitutivos para estudiar el comportamiento no lineal del hormigón. Estos consideran plasticidad, mecánica de daño y una combinación de plasticidad y mecánica de daño. Los modelos basados en plasticidad permiten modelar el comportamiento del hormigón bajo un estado triaxial de tensiones. La separación de las deformaciones en su parte elástica y plástica representa en forma realista las deformaciones que se observan en compresión confinada, y puede describirse adecuadamente el proceso de descarga, Willam y Warnke (1974); Pramono y Willam (1989); Etse y Willam (1994); Menétrey y Willam (1995); Pivonka (2001b); Grassl et al. (2002); Papanikolaou y Kappos (2007); Cy Papanikolaou (2008); Folino y Etse (2012).

Los modelos de mecánica de daño se basan en el concepto de reducción gradual de la rigidez. Para modelos continuos de la mecánica de daño basados en deformaciones, el proceso de evaluación de las tensiones es explícito, lo cual permite una determinación directa del estado tensional, sin la realización de un proceso de cálculo iterativo. Estos modelos describen adecuadamente la degradación de la rigidez en tracción y bajo cargas bajas de compresión confinada concordando con los resultados observados en los ensayos de laboratorio, Kachanov (1980); Ortiz (1985); Resende (1987); 
Mazars y Pijaudier-Cabot (1989); Carol et al. (2001); Tao y Phillips (2005); Voyiadjis y Kattan (2009).

Sin embargo, los modelos basados en plasticidad no pueden describir la reducción de la rigidez frente a la descarga observada en ensayos reales. Por otro lado, los modelos de daño continuo no permiten considerar las deformaciones irreversibles, y se encuentran principalmente limitados a estados de tracción y bajos valores de compresión confinada. Por este motivo, la combinación de plasticidad y mecánica de daño continuo permiten modelar en forma adecuada el comportamiento no lineal del hormigón, ya que en este caso se tienen en cuenta las deformaciones irreversibles luego del proceso de descarga y la degradación de la rigidez, Grassl et al. (2013); Omidi et al. (2010).

Los modelos de daño con plasticidad utilizan las ecuaciones de la teoría de plasticidad e incorporan la variable de daño. Las primeras se dividen en los siguientes tres grupos:

1) Descomposición de las deformaciones en su parte elástica y plástica, con las ecuaciones de Hooke que relacionan las deformaciones elásticas con las tensiones aplicadas.

2) Criterio de plasticidad, definido a través de la función de fluencia. Permite determinar bajo qué condiciones el comportamiento es elástico o plástico.

3) La regla de flujo, definida como un parámetro que establece cómo se incrementa la deformación plástica.

Distintos autores han propuesto modelos de plasticidad con daño, Ju (1989); Lee y Fenves (1998); Jason et al. (2006); Grassl y Jirásek (2006); Nguyen y Houlsby (2008); Nguyen y Korsunsky (2008); Voyiadjis et al. (2008); Grassl (2009); Sánchez et al. (2011); Valentini y Hofstetter (2013). A continuación se describirán brevemente cada uno de ellos.

$\mathrm{Ju}$ (1989) propone una serie de modelos energéticos de daño isótropo y anisótropo para caracterizar la iniciación y crecimiento de microfisuras en materiales dúctiles y frágiles.

Lee y Fenves (1998) desarrollaron un modelo para el hormigón sujeto a cargas cíclicas utilizando conceptos de daño basados en energía de fractura y degradación de la rigidez en mecánica del continuo. Definen dos variables de daño, una en tracción y otra en compresión, e introducen una función de fluencia con múltiples variables de endurecimiento para considerar diferentes estados de daño. Las funciones uniaxiales de resistencia se factorizan en dos partes, una de ellas corresponde a la tensión efectiva y la otra a la degradación elástica de la rigidez. Las ecuaciones constitutivas para la respuesta elastoplástica están desacopladas con respecto a la respuesta de degradación por daño, lo cual representa una ventaja en la implementación numérica. La 
función de resistencia para las tensiones efectivas se utiliza para controlar la evolución de la superficie de fluencia. El modelo de degradación para simular el efecto del daño en la rigidez elástica y la recuperación durante el proceso de apertura y cierre de fisuras es escalar.

Jason et al. (2006) proponen una ley constitutiva de daño elasto-plástico en la cual el daño isótropo es responsable del ablandamiento y del decrecimiento en la rigidez, mientras que el endurecimiento plástico considera el desarrollo de las deformaciones irreversibles y el comportamiento en deformaciones volumétricas por compresión. Para la validación del modelo se realizaron ensayos de tracción, compresión bajo carga cíclica y compresión triaxial. Además presentan una aplicación estructural del modelo en columnas de hormigón contenidas en tubos de acero.

Grassl y Jirásek (2006) presentan un modelo triaxial de daño con plasticidad para el estudio de la falla del hormigón. En este modelo la parte plástica se basa en tensiones efectivas y el modelo de daño es conducido por la deformación plástica. Los autores demuestran que para modelos de daño con plasticidad en los que la parte plástica está basada en tensiones efectivas, la unicidad local está siempre garantizada, lo cual no puede asegurarse en modelos con la parte plástica basada en tensiones nominales. El modelo permite capturar parcialmente la reducción en la rigidez por corte debido a cargas previas de compresión, y emplea a su vez un esquema de integración implícita. Para la validación se compararon los resultados obtenidos con resultados experimentales de tracción uniaxial y compresión triaxial. Además se aplicó al análisis estructural de columnas de hormigón armado.

Nguyen y Houlsby (2008) desarrollan un modelo acoplado de plasticidad con daño en tracción y compresión, cuyo comportamiento se describe a partir de dos potenciales energéticos. Para las respuestas en tracción y compresión se utilizan dos criterios de daño separados, y una función de fluencia con endurecimiento múltiple. Los parámetros del modelo se determinan a partir de ensayos de laboratorio en hormigón. Luego Nguyen y Korsunsky (2008) propusieron un procedimiento novedoso para la identificación de los parámetros del modelo de plasticidad con daño, utilizando la separación de la energía total disipada en partes aditivas correspondientes a distintos mecanismos de disipación. Además se establece la relación entre los parámetros locales y no locales ayudando a obtener respuestas del modelo consistentes con la energía de fractura del material. La aplicación del modelo y del procedimiento de calibración propuesto se aplica a una serie de pruebas en estructuras reales.

El modelo propuesto por Voyiadjis et al. (2008) considera dos tensores de daño anisótropos y dos criterios de daño para describir la degradación del hormigón bajo cargas de tracción y compresión. El tensor de tensiones totales se descompone en tracción y compresión. El criterio de fluencia considera la descomposición espectral del tensor de tensiones y utiliza múltiples reglas de endurecimiento. El criterio de fluencia se utiliza conjuntamente con 
el criterio de daño para simular el comportamiento del hormigón. Además se emplea una regla de flujo no asociado para considerar la dilatación del hormigón al tratarse de un material friccional. Se utiliza el concepto de energía libre de Helmholtz para derivar de manera consistente los potenciales de disipación por daño y plasticidad y permitir leyes de evolución para los diferentes parámetros de endurecimiento. La evolución de los dos tensores de daño se tiene en cuenta a través de la energía de fractura.

Grassl (2009) presenta un modelo de plasticidad con daño para la descripción de la fractura del hormigón simple, considerando dos aproximaciones: un modelo local que comprende el ajuste del módulo de ablandamiento y un modelo no local basado en un promedio espacial de la historia de las variables consideradas. Estas aproximaciones se aplican al análisis de una barra de hormigón sometida a tracción uniaxial y a un ensayo de flexión en tres puntos. Los resultados obtenidos son independientes de la malla para los dos casos analizados. Sin embargo el modelo no local presenta dependencia con las condiciones de contorno.

Sánchez et al. (2011) presentan un modelo constitutivo macroscópico fenomenológico para la simulación numérica del comportamiento del hormigón bajo estados de tensión triaxiales. El modelo permite capturar la fuerte influencia de la tensión media en los mecanismos de falla del hormigón. El comportamiento mecánico en diferentes dominios del espacio de tensiones se describe mediante una respuesta del material de tipo dúctil o cuasifrágil. Así, para estados de tensiones de tracción, se considera un modelo de daño isótropo continuo con ablandamiento de las deformaciones. En este contexto se utiliza una técnica de regularización para evitar los problemas de valores de contorno mal condicionados inducidos por la ley de ablandamiento. Cuando el estado de tensión es de confinamiento, la respuesta del material es gobernada por un modelo plástico basado en la teoría clásica del flujo plástico. El criterio de fluencia utilizado depende de los tres invariantes de las tensiones, se utiliza una ley isótropa de endurecimiento de las deformaciones y una ley de flujo no asociado. El algoritmo fue validado mediante ensayos de laboratorio.

Valentini y Hofstetter (2013) evaluaron el modelo de plasticidad presentado por Etse y Willam (1994) y modificado por Pivonka (2001a) y el modelo de daño con plasticidad propuesto por Grassl y Jirásek (2006), en el cual modificaron la ley de evolución de la variable de daño, para describir el comportamiento en tres dimensiones del hormigón. A partir del estudio concluyeron que el modelo de daño con plasticidad presentaba una performance superior. Luego aplicaron este último a la simulación numérica de distintos ensayos en 3D, de problemas benchmark en los que se combinan esfuerzos de flexión y torsión y compresión y corte. Finalmente simularon una presa arco de hormigón utilizando elementos finitos. 


\subsubsection{Método de los Elementos Finitos Extendido (XFEM)}

La modelación del proceso de propagación de fisuras utilizando una malla de elementos finitos resulta ser un problema complejo debido a que debe discretizarse la discontinuidad y la punta de la fisura en forma precisa, y además incluirse modificaciones en la topología de la malla que permitan simular el proceso de propagación de la fisura a medida que avanza el análisis. Esto representa una operación costosa, desde el punto de vista computacional, pero también puede tener un impacto negativo en la calidad de los resultados.

En este contexto se ha propuesto un método en el que el crecimiento de las fisuras puede modelarse sin remallado, en el cual las fisuras se representan a través de funciones de enriquecimiento. Este método se conoce como Método de los Elementos Finitos Extendido (XFEM, por sus siglas en inglés) y fue propuesto originalmente por Belytschko y Black (1999).

XFEM representa una extensión del Método de los Elementos Finitos convencional basada en el concepto de partición de la unidad, Melenk y Babûska (1996); Duarte y Oden (1996), según el cual un dominio se divide en subdominios solapados $\Omega_{I}$, cada uno de los cuales está asociado con una función $N_{I}(x)$, distinta de cero solo en $\Omega_{I}$ y que presenta la siguiente propiedad

$$
\sum_{I=1}^{N} N_{I}(x)=1 \text { en } \Omega
$$

En otras palabras las expresión 2.8 significa que la suma de las funciones de forma debe ser igual a uno.

La importancia del método de la partición de la unidad radica en que generaliza las aproximaciones por elementos finitos al presentar un medio para incluir soluciones locales de los problemas de valores de contorno dentro de la formulación de elementos finitos, Sukumar y Perévost (2003). A su vez satisface algunas propiedades clave, las cuales lo convierten en una herramienta poderosa para el enriquecimiento local dentro del marco de los elementos finitos, Sukumar y Perévost (2003):

* Incorporación de funciones básicas específicas para aproximar mejor la solución.

Aplicación automática de la condición de continuidad (ajustando aproximaciones a prueba y error).

* Modelación de puntos o líneas singulares, así como discontinuidades de superficie, sin la necesidad de que las superficies discontinuas estén alineadas con la malla de elementos finitos.

Se propone un enriquecimiento de los elementos finitos cercanos a la punta de la fisura empleando la expresión 2.9, Belytschko y Black (1999):

$$
F_{\alpha}(x)=\sqrt{r} \cos \left(\frac{\theta}{2}\right), \sqrt{r} \sin \left(\frac{\theta}{2}\right), \sqrt{r} \sin \left(\frac{\theta}{2}\right) \sin (\theta), \sqrt{r} \cos \left(\frac{\theta}{2}\right) \sin (\theta)
$$

Ing. M. Paula Zappitelli 
donde $(r, \theta)$ es un sistema de coordenadas polares con su origen en la punta de la fisura y $\theta=0$ es tangente a la fisura en la punta, ver Figura 2.15.

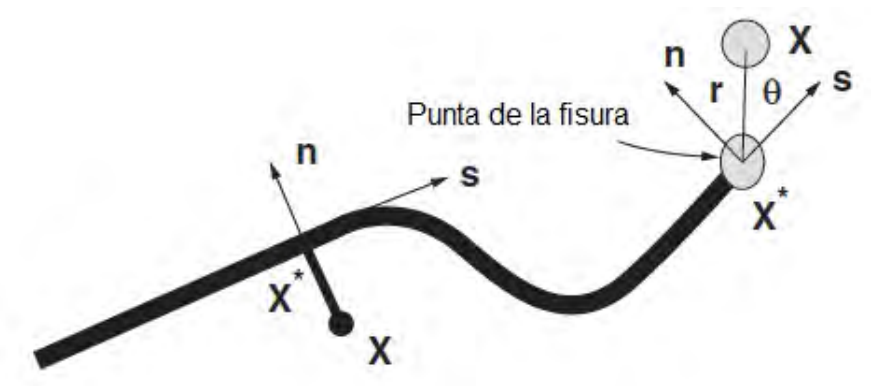

Figura 2.15: Coordenadas normal y tangencial en una fisura aplicando método XFEM, ABAQUS (2013a)

Sin el enriquecimiento, debe realizarse una malla considerablemente refinada cerca de la punta de la fisura, y además adaptarla a la forma de las caras de la fisura. Los nodos seleccionados alrededor de la fisura presentan grados de libertad adicionales asociados a las funciones de enriquecimiento.

Más tarde tuvo lugar una notable mejora y progreso en la modelación de fisuras discretas sin la necesidad de remallado al proponer la función generalizada de Heviside como un medio para modelar la fisura más allá de su punta, con reglas simples para introducir la discontinuidad y los enriquecimientos en la punta de la misma, Moës et al. (1999). La función de Heviside, $H(x)$, es una función discontinua a través de la superficie de la fisura y constante a cada lado de la misma, tomando el valor +1 en un lado y -1 en el otro. Este avance provee una herramienta computacional precisa y robusta para el modelado de discontinuidades independientemente de la geometría de la fisura, Sukumar y Perévost (2003).

XFEM puede emplearse tanto para el modelado de fisuras estacionarias como de fisuras que se propagan. El vector desplazamiento se expresa como se indica en 2.10 :

$$
\mathbf{u}=\Sigma_{I=1}^{N} N_{I}(x)\left[\mathbf{u}_{I}+H(x) \mathbf{a}_{I}+\Sigma_{\alpha=1}^{4} F_{\alpha}(x) \mathbf{b}_{I}^{\alpha}\right]
$$

donde $N_{I}(x)$ son las funciones de forma convencionales, $\mathbf{u}_{I}$ es el vector de desplazamientos nodales asociado a la parte continua de la solución de elementos finitos. El segundo término de la expresión 2.10 es el producto entre el vector grados de libertad nodales enriquecidos, $\mathbf{a}_{I}$, y la función de Heviside, $H(x)$. Finalmente, el tercer término es el producto entre el vector grados de libertad nodales enriquecidos, $\mathbf{b}_{I}^{\alpha}$, y las funciones elásticas asintóticas asociadas en la punta de la fisura, $F_{\alpha}(x)$, definidas en 2.9. El primer término de la expresión 2.10 se aplica a todos los nodos del modelo, el segundo término es válido para los nodos cuyas funciones de forma son atravesadas por 
el interior de una fisura, y el tercer término es utilizado para nodos cuyas funciones de forma son cortadas por la punta de la fisura, Belytschko y Black (1999). La función de Heviside presenta el siguiente comportamiento:

$$
H(x)= \begin{cases}1 & \text { si }\left(\boldsymbol{x}-\boldsymbol{x}^{*}\right) \boldsymbol{n} \geq 0 \\ -1 \quad \text { caso contrario }\end{cases}
$$

en este caso $\boldsymbol{x}$ es un punto de prueba (punto de Gauss), $\boldsymbol{x}^{*}$ es el punto en la fisura más cercano a $\boldsymbol{x}$ y $\boldsymbol{n}$ es la normal exterior unitaria a la fisura en $\boldsymbol{x}^{*}$.

Para estudiar propagación de fisuras una variante de XFEM consiste en considerar el Método de los segmentos cohesivos junto con el Método de los nodos fantasma, lo cual permite simular la iniciación y propagación de fisuras a lo largo de un camino arbitrario en el material dependiente de la solución, ya que la propagación de la fisura no está atada a los límites impuestos por el mallado. En este caso la función que modela la singularidad en la punta de la fisura no es necesaria, solamente se considera el salto en el desplazamiento a través del elemento fisurado. Por lo tanto, la fisura debe propagarse a través de todo el elemento y así se evita la necesidad de modelar la singularidad tensional, Remmers et al. (2008).

El Método de los nodos fantasma es otra forma de expresar el campo de desplazamientos de XFEM, Hansbo y Hansbo (2004); Areias y Belytschko (2005). Según el mismo los nodos fantasma, los cuales se encuentran superpuestos a los nodos reales, se introducen para representar la discontinuidad en los elementos fisurados, es decir, que el elemento discontinuo es reemplazado por dos elementos con nodos fantasma adicionales o grados de libertad fantasma, Song et al. (2006). Cuando el elemento está intacto cada nodo fantasma está completamente vinculado a su correspondiente nodo real, pero cuando el elemento es atravesado por una fisura, el elemento fisurado se divide en dos partes. Cada parte está formada por una combinación de nodos fantasma y nodos reales, dependiendo de la orienteación de la fisura, como puede observarse en la Figura 2.16.

Para tener un conjunto de bases de interpolación completa, la parte del elemento fisurado que pertenece al dominio real, $\Omega_{0}$, se extiende al dominio fantasma, $\Omega_{p}$. Así el desplazamiento en el dominio real puede interpolarse utilizando los grados de libertad para los nodos en el dominio fantasma. El salto en el campo de desplazamientos se realiza integrando solo sobre el área desde el nodo real hacia la fisura, es decir, $\Omega_{0}^{+}$y $\Omega_{0}^{-}$, Song et al. (2006).

Por lo expuesto anteriormente puede decirse que solo se necesitan pequeñas modificaciones en los programas explícitos de elementos finitos para implementar esta formulación para elementos con fisuras. Las funciones de forma asociadas a un elemento fisurado son idénticas a las funciones de forma de un elemento intacto, lo cual lleva a simplificaciones de implementación en los códigos existentes, Song et al. (2006).

La magnitud de la separación entre los nodos reales y los nodos fantasma

Ing. M. Paula Zappitelli 


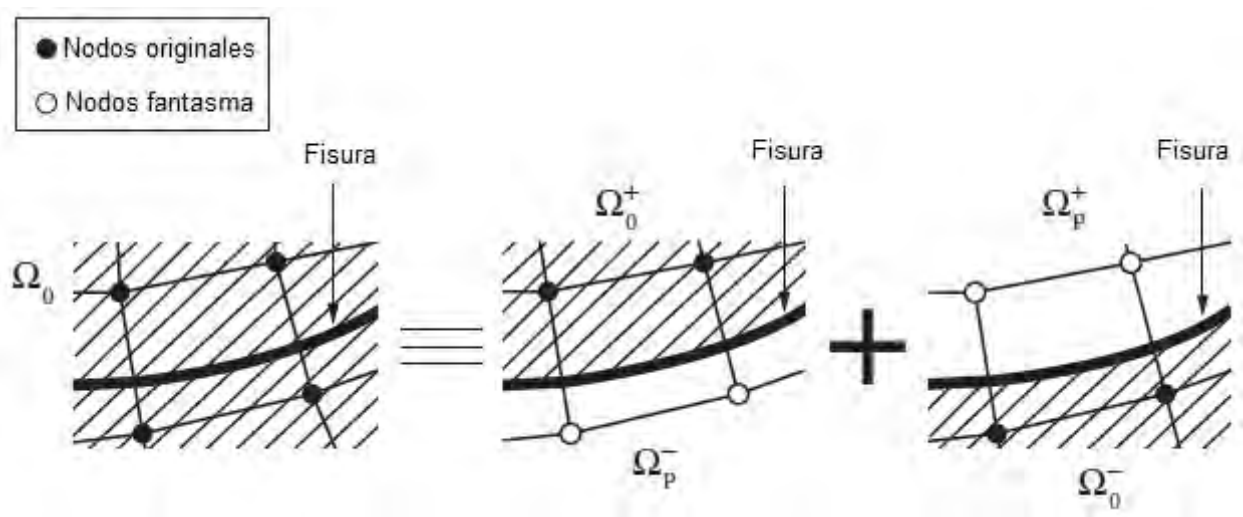

Figura 2.16: Principio del Método de los nodos fantasma, ABAQUS (2013a)

es gobernada por una ley cohesiva hasta que la magnitud de la resistencia cohesiva del elemento fisurado es cero, momento a partir del cual el nodo real y el nodo fantasma se mueven de forma independiente, Song et al. (2006); Remmers et al. (2008). 


\section{Capítulo 3}

\section{Algoritmo desarrollado y modelo numérico aplicado para el estudio del proceso de fisuración en estructuras de hormigón}

\subsection{Introducción}

Si se realizan ensayos mecánicos en muestras de hormigón nominalmente idénticas se observarán variaciones en los valores de las propiedades obtenidas para cada una de dichas muestras. Los factores que contribuyen a esta variabilidad pueden agruparse en las siguientes categorías, Mindess et al. (2003):

- Materiales: La variabilidad propia del cemento; en la granulometría, contenido de humedad, composición mineral, propiedades físicas y forma de las partículas de los agregados y las adiciones utilizadas.

- Producción: Tipo de planta de producción y equipamiento, método de transporte, procedimientos y mano de obra utilizada para producir y colocar el hormigón.

- Ensayos: Procedimiento para obtener las muestras, el moldeado y curado de las probetas y los procedimientos de ensayo utilizados.

La resistencia del hormigón puede representarse mediante diferentes funciones de distribución de probabilidad, como por ejemplo la Gaussiana o distribución normal. La adopción de esta distribución es más bien fenomenológica, Tumidajski et al. (2006), ya que si muestras nominalmente idénti- 
CAPítulo 3. Algoritmo desarrollado y modelo numérico aplicado para el estudio del proceso de fisuración en estructuras de hormigón

cas de hormigón se ensayan, los valores de resistencia obtenidos siguen esta distribución.

En la expresión 3.1 se indica la función densidad de probabilidad para la distribución Gaussiana:

$$
y=\frac{1}{s \sqrt{2 \pi}} \exp ^{-\left[\frac{(x-\mu)^{2}}{2 s^{2}}\right]}
$$

donde $\mu$ representa el valor medio y $s$ la desviación estándar de la muestra.

La resistencia de los materiales frágiles sigue una distribución de Weibull debido a que la rotura es provocada por la falla de un elemento representativo del volumen (REV, por sus siglas en inglés), en concordancia con la estadística de valores extremos y el modelo del eslabón débil en una cadena infinita de REVs. El tamaño de los REVs es despreciable en comparación con el tamaño de la estructura, Le et al. (2012).

El hormigón es un material cuasi-frágil, por esta razón antes de que se produzca la falla tienen lugar una liberación de energía y una redistribución de tensiones, Baẑant et al. (1991); Baẑant y Novak (2000a,b), el tamaño de la zona en proceso de fractura no es despreciable en comparación con la dimensión representativa del elemento en estudio, Baẑant y Pang (2007) y la rotura por compresión en el hormigón se origina debido al lento crecimiento de las fisuras, en lugar de la rápida e inestable propagación de microfisuras, como es de esperarse en la teoría del eslabón débil, Yip et al. (1995); Carpinteri et al. (1999). A pesar de lo expuesto anteriormente, Tumidajski et al. (2006) muestran experimentalmente que la distribución de Weibull puede aplicarse a la resistencia del hormigón. También en Yang et al. (2009), se utiliza la distribución de Weibull para estudiar problemas de fisuración en muestras de hormigón.

La función densidad de probabilidad para la distribución de Weibull se indica en la expresión 3.2:

$$
\begin{array}{ccc}
y & =\frac{m}{\sigma_{0}} \frac{-V}{V_{0}}\left(\frac{x}{\sigma_{0}}\right)^{(m-1)}\left(-\exp ^{\frac{-V}{V_{0}}\left(\frac{x}{\sigma_{0}}\right)^{m}}\right) x \geq 0 \\
y & =0 & x<0
\end{array}
$$

donde $m$ es el parámetro de forma, $\sigma_{0}$ el parámetro de escala, $V$ es el volumen del cuerpo y $V_{0}$ un volumen representativo del material, es decir, el volumen mínimo de una probeta a escala de laboratorio, tal que los resultados obtenidos a partir de esa probeta pueden considerarse representativos de lo que sucede en el continuo, van Mier (1997). El parámetro de forma describe la manera en que se distribuyen los datos. Por ejemplo, un parámetro de forma 3, se aproxima a la curva correspondiente a una distribución normal. Un valor bajo de dicho parámetro, por ejemplo 1, permite obtener una curva con asimetría hacia la derecha, por el contrario un parámetro de forma alto, por ejemplo 10, origina una curva con asimetría hacia la izquierda. El 
parámetro de escala es el percentil 63.2 de los datos y define la posición de la curva de Weibull respecto del valor umbral, lo cual es similar a la manera en que la media define la posición de una curva con distribución normal. Por ejemplo, un parámetro de escala igual a 20, indica que el $63.2 \%$ de las probetas tendrán una resistencia $20 \mathrm{MPa}$ mayor que el valor umbral. El valor umbral describe un desplazamiento de la distribución alejándose del 0 . Un valor umbral negativo desplaza la distribución hacia la izquierda, mientras que un valor umbral positivo desplaza la distribución hacia la derecha. Todos los datos deben ser mayores que el valor umbral, Moreno et al. (2011). En esta Tesis se adoptó una distribución de Weibull de dos parámetros, por lo que el valor umbral se consideró igual a 0 .

La Figura 3.1 (izquierda) muestra la función densidad de probabilidad para la distribución de Gauss y para la distribución de Weibull.
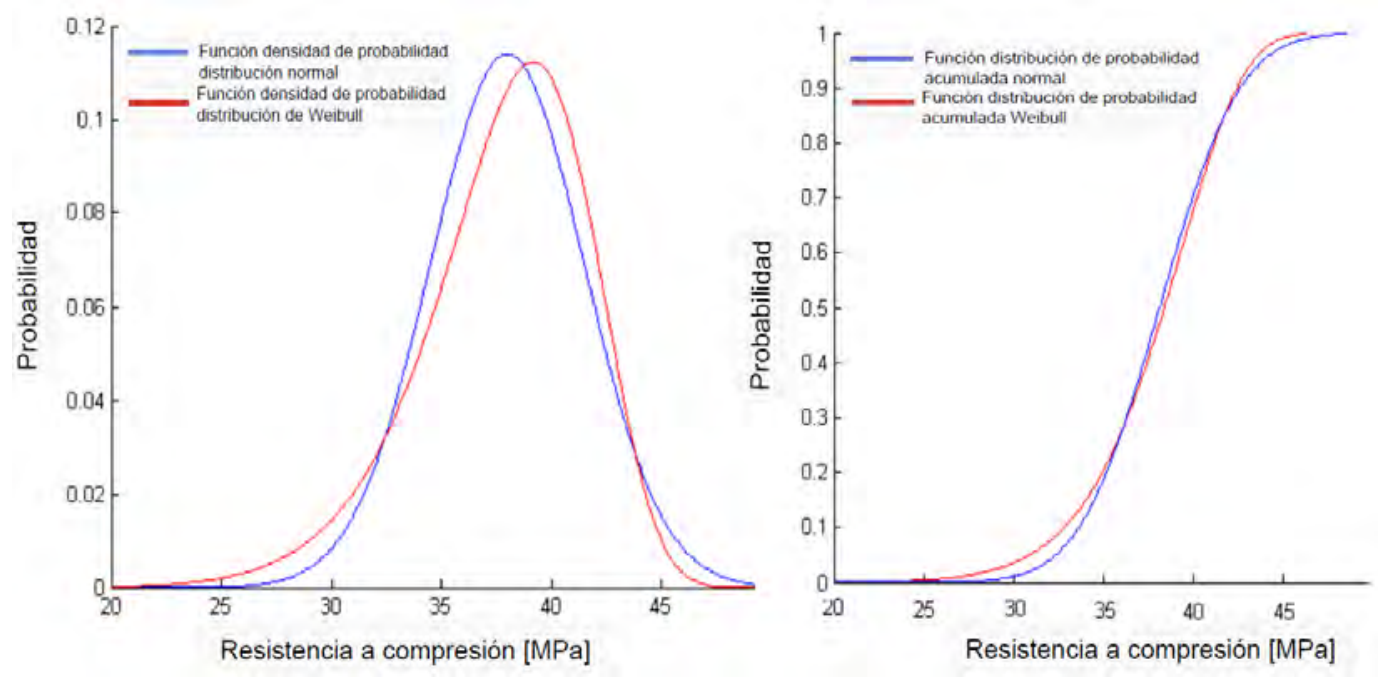

Figura 3.1: Función densidad de probabilidad de Gauss y Weibull (izquierda). Comparación entre las distribuciones acumuladas de Gauss y Weibull (derecha)

Si se compara la función distribución acumulada para ambas distribuciones, se observa que la diferencia entre las mismas es pequeña en la parte central y difícilmente detectable en histogramas experimentales, ver Figura 3.1 (derecha). Pero para probabilidades extremadamente bajas las diferencias en el decaimiento exponencial entre las distribuciones de Gauss y de Weibull es muy importante, Baẑant et al. (2007); Baẑant y Pang (2007).

Generalmente en los modelos numéricos al definir las propiedades mecánicas del hormigón se consideran sus valores medios o característicos en lugar de la función de distribución de probabilidad representativa de dicho parámetro.

Por otro lado, en problemas de fractura en hormigón, con propiedades 
CAPítulo 3. Algoritmo desarrollado y modelo numérico aplicado para el estudio del proceso de fisuración en estructuras de hormigón

mecánicas y estados tensionales uniformes, no es posible localizar una fisura empleando un modelo de daño continuo.

Otro aspecto importante a considerar es la variación en el tiempo de las propiedades mecánicas del hormigón debido a que este material evoluciona de un estado fresco a un estado endurecido. Para un correcto estudio de la fisuración en el hormigón a edad temprana, es decir a las pocas horas o días del hormigonado, es de fundamental importancia considerar esta variación, ya que la resistencia del hormigón aún se encuentra evolucionando. Para ello pueden emplearse ecuaciones obtenidas de códigos como el CEB-FIB (1993), o mediante el ajuste a partir de los resultados obtenidos en ensayos de laboratorio. El algoritmo desarrollado tiene en cuenta este aspecto a través de la incorporación de las mencionadas ecuaciones.

En este contexto se ha desarrollado un algoritmo en Matlab que permite asignar dentro del modelo numérico las propiedades mecánicas del material, de acuerdo con las funciones de distribución descriptas previamente. El algoritmo emplea como criterio la heterogeneidad del hormigón, para determinar zonas de fisuración preferentes en estructuras inicialmente sin defectos y sometidas a un estado tensional uniforme.

\subsection{Algoritmo desarrollado para la asignación alea- toria de propiedades mecánicas del hormigón}

El funcionamiento del algoritmo consiste en agrupar los elementos finitos de la malla en sets (conjuntos), y en la asignación aleatoria a cada uno de ellos de un valor de las propiedades mecánicas de acuerdo con una función de distribución de probabilidad. Para realizar lo indicado anteriormente el algoritmo utiliza dos tipos de mallas:

- Una malla de elementos finitos convencional, obtenida con cualquier código de análisis por el Método de los Elementos Finitos, en este caso Abaqus/Standard. Esta malla presenta la forma geométrica de la estructura que se está estudiando, y puede ser regular o irregular, como se observa en la Figura 3.2 (izquierda).

- Una malla de tipo rectangular conformada por un conjunto de nodos y denominada "malla de material". La separación de los nodos se define como tres veces el tamaño máximo del agregado. Esto surge al considerar que el tamaño de la zona en proceso de fractura se puede adoptar como el tamaño de un REV, el cual es aproximadamente igual al triple del tamaño de la máxima inhomogeneidad presente en el material, por ejemplo el tamaño máximo del agregado en el caso del hormigón, Baẑant y Pang (2007). En la Figura 3.2 (derecha) se muestra este tipo de malla. 
3.2. Algoritmo desarrollado para la asignación aleatoria de propiedades mecánicas del hormigón

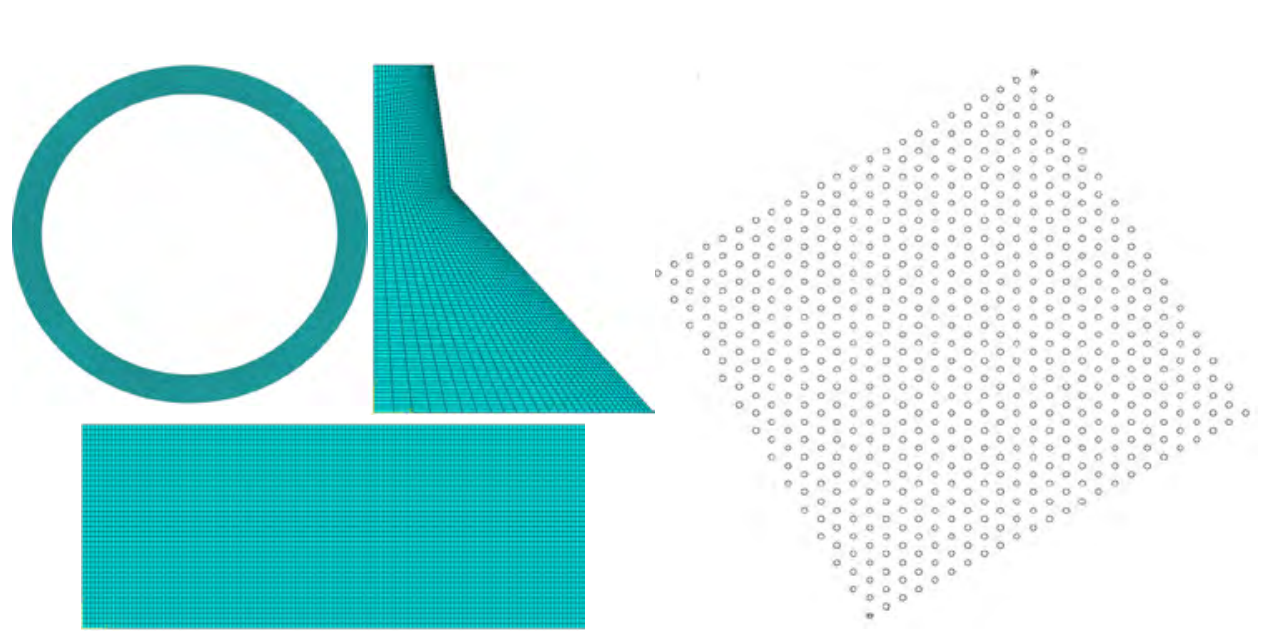

Figura 3.2: Malla de elementos finitos convencional (izquierda). Malla de material (derecha) utilizadas por el algoritmo desarrollado
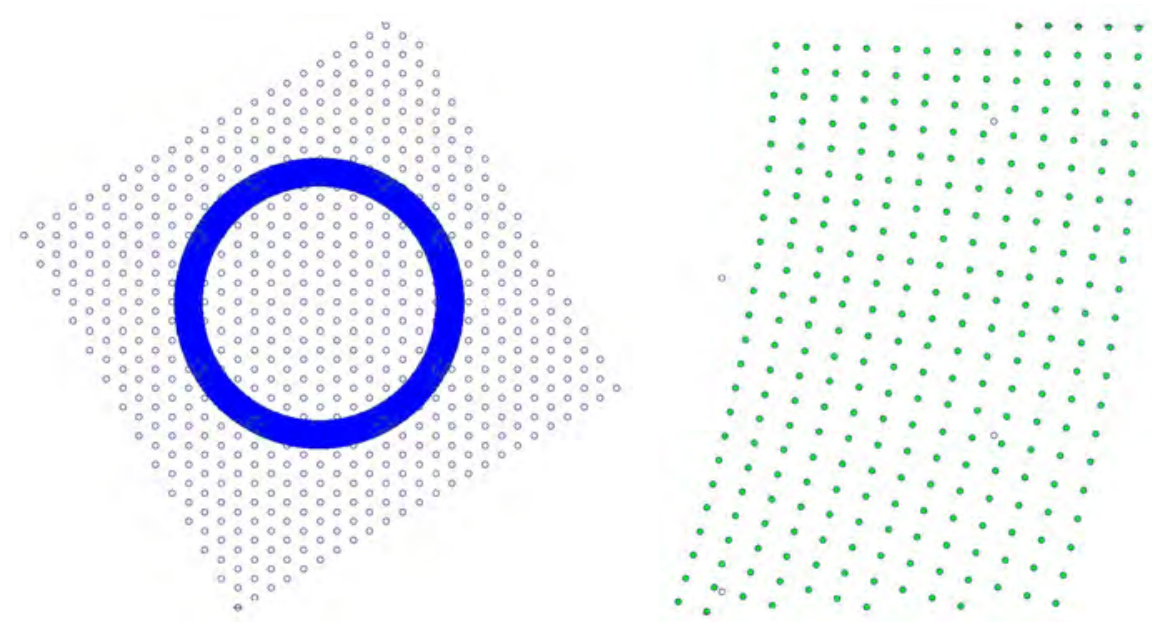

Figura 3.3: Superposición de la malla de material y la malla de elementos finitos

El algoritmo calcula la posición de los centroides de cada uno de los elementos de la malla de elementos finitos, y luego superpone la malla de elementos finitos y la malla de material. Los centroides de los elementos finitos se agrupan alrededor de los nodos de la malla de material, definiendo así regiones con un tamaño igual a tres veces el tamaño máximo del agregado, como se mencionó previamente, Baẑant y Pang (2007), ver Figura 3.3. Para evitar la superposición de elementos entre las regiones se determina la mínima distancia entre los centroides de la malla de elementos finitos y los nodos de la malla de material. Las regiones así determinadas constituyen diferentes sets de elementos finitos y a cada una de ellas se le asigna un número 
CaPítulo 3. Algoritmo desarrollado y modelo numérico aplicado para el estudio del proceso de fisuración en estructuras de hormigón

aleatorio.

Los números aleatorios son una secuencia de valores que adopta una variable aleatoria asociada a un proceso físico. Por ejemplo, el conjunto de los intervalos de tiempo en los cuales un contador Geiger colocado frente a una muestra radioactiva emite una señal constituye una secuencia de números aleatorios. Sin embargo, en términos prácticos no resulta viable utilizar este tipo de procesos en una simulación numérica. Por este motivo se recurre a algoritmos que originan una secuencia de números que emulan números aleatorios. Sin embargo, se plantea el interrogante de si es válido utilizar algoritmos, los cuales son necesariamente deterministas, para obtener valores que se suponen en alguna medida impredecibles. Por ello es que se emplean pruebas estadísticas que permiten comparar los números aleatorios obtenidos con los generadores con un conjunto de "auténticos números aleatorios". Si los primeros resultan indistinguibles de los segundos respecto de un cierto conjunto de pruebas, puede considerarse que se tiene un buen conjunto de números aleatorios en relación a éstas. Una de ellas es la denominada prueba de distancia, en la que se cuenta el número de dígitos que aparecen entre ocurrencias sucesivas de un mismo dígito. Por ejemplo, en la secuencia 0,1,5,6,9,0 hay una distancia de 4 unidades entre los dos 0, Cannas (2018); Gentle (2004); Knuth (1997).

Algunos procesos físicos a partir de los cuales pueden obtenerse números aleatorios verdaderos son el ruido térmico por agitación de portadores, fuentes caóticas y el ruido atmosférico. Dentro de los generadores de números aleatorios se encuentran los generadores congruenciales lineales, en los que a partir de los parámetros $r_{0}, m, a$ y $c$, siendo $r_{0}$ la semilla, $m$ el módulo y $a$ y $c$ dos números enteros, la secuencia de números aleatorios enteros $r_{i}$, con distribución uniforme entre 0 y $m$ se construye a partir de $r_{0}$ mediante la expresión siguiente:

$$
r_{i}=\left(a * r_{i-1}+c\right) \bmod m
$$

Este generador presenta un ciclo cuyo máximo valor posible será $m$, el cual constituye el mayor entero que admite el lenguaje, ya que una vez que se repita un valor de la secuencia, la misma se repetirá de manera completa. Es de fundamental importancia la elección de los enteros $a$ y $c$, porque una mala elección produce ciclos mucho menores que $m$. El generador congruencial lineal no es muy bueno, porque excepto por su rapidez, presenta ciclos relativamente pequeños y una alta correlación entre distintos valores de las secuencias, lo que implica falta de independencia estadística, Cannas (2018).

Otro generador pseudo aleatorio es el Mersenne Twister, y es el utilizado en el algoritmo desarrollado. En este caso se busca aprovechar la naturaleza binaria de las computadoras, trabajando con operaciones bit a bit y obteniendo generadores más rápidos. En este generador se trabaja con el tamaño de una palabra, definido como $\omega$, por ejemplo 32 bits, y se generan números 
3.2. Algoritmo desarrollado para la asignación aleatoria de propiedades mecánicas del hormigón

enteros entre 0 y $2^{\omega}-1$.

La mayoría de los generadores estándar producen números aleatorios con distribución uniforme, es decir, que para una secuencia $\left(u_{1}, u_{2} \ldots ., u_{n}\right)$ se cumple que los $u_{i}$ se distribuyen uniformemente en [0,1], que los pares $\left(u_{i}, u_{i+1}\right)$ se distribuyen uniformemente en $[0,1] \mathrm{x}[0,1]$ y las n-uplas $\left(u_{i}, u_{i+1}, \ldots, u_{n+i}\right)$ se distribuyen uniformemente en $[0,1]^{n}$, Gentle (2004). Por este motivo es necesario determinar un método para que éstos adopten otro tipo de distribución, por ejemplo una distribución gaussiana o de Weibull. Uno de esos métodos, denominado método de la inversa acumulada, consiste en considerar que si a la variable aleatoria $X$ se le aplica una función $H(X)=G^{-1}(X)$, con $G(X)$ estrictamente creciente, se genera una nueva variable aleatoria $Y$, por lo que el procedimiento para hallar la función distribución de probabilidad de $Y$ es el siguiente:

$$
\begin{aligned}
P(Y \leq y) & =P(H(X) \leq y)=P\left(G^{-1}(X) \leq y\right)= \\
& =P(X \leq G(y)) \equiv F_{d a}(G(y))=G(y)
\end{aligned}
$$

Por lo que la función de distribución de probabilidad de la variable aleatoria $Y$ es la inversa de la función que se le aplicó a la variable aleatoria $X$. Esto significa que si se elige como $H(X)$ a la inversa de la acumulada de una variable aleatoria con distribución arbitraria se obtendrá una variable aleatoria con la distribución elegida, Gentle (2004), Knuth (1997). En la Figura 3.4 se muestra lo referido anteriormente en un diagrama de flujo.

El algoritmo desarrollado realiza un sorteo de números aleatorios empleando el generador de números aleatorios Mersenne Twister. Los números aleatorios obtenidos siguen una distribución uniforme, por lo que para lograr que los mismos sigan una distribución de Gauss o de Weibull se los debe evaluar en la inversa de la función de probabilidad elegida, de acuerdo a lo explicado anteriormente. Luego a cada uno de los sets de elementos definidos previamente, asociados a un determinado número aleatorio, se le asigna un valor de la propiedad obtenida de la función de distribución de probabilidad. El algoritmo genera un archivo de texto con los sets de elementos creados.

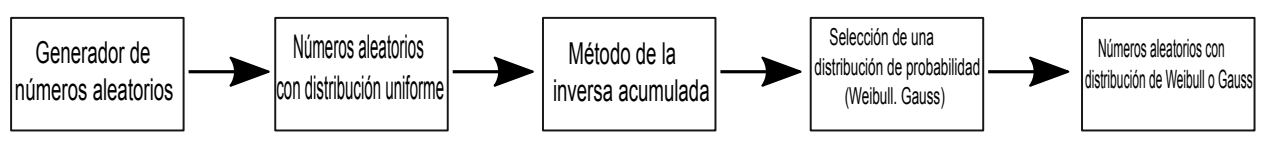

Figura 3.4: Método de la inversa acumulada para obtener números aleatorios con una distribución de probabilidad distinta de la uniforme

Una vez que se obtuvo una distribución no uniforme de la propiedad en estudio, es necesario definir la variación de la misma con el tiempo. Para ello los valores obtenidos anteriormente para cada sorteo de números aleatorios, se transforman en datos de entrada para calcular la variación temporal de la propiedad en estudio. La salida del algoritmo a este nivel es un archivo 
CaPítulo 3. Algoritmo desarrollado y modelo numérico aplicado para el estudio del proceso de fisuración en estructuras de hormigón

de texto con las propiedades mecánicas del material variables en el tiempo asignadas a cada set de elementos. Finalmente ambos archivos de texto generados con el algoritmo se incluyen en el archivo de entrada de Abaqus /Standard. Este procedimiento se repite todas las veces que el usuario lo desee, obteniendo distribuciones aleatorias de las propiedades mecánicas del hormigón para la función de distribución de probabilidad elegida. A partir de los resultados obtenidos se puede realizar un análisis estadístico del fenómeno en estudio. En la Figura 3.5 se observa la asignación de propiedades mecánicas en una geometría elíptica utilizando el algoritmo desarrollado.

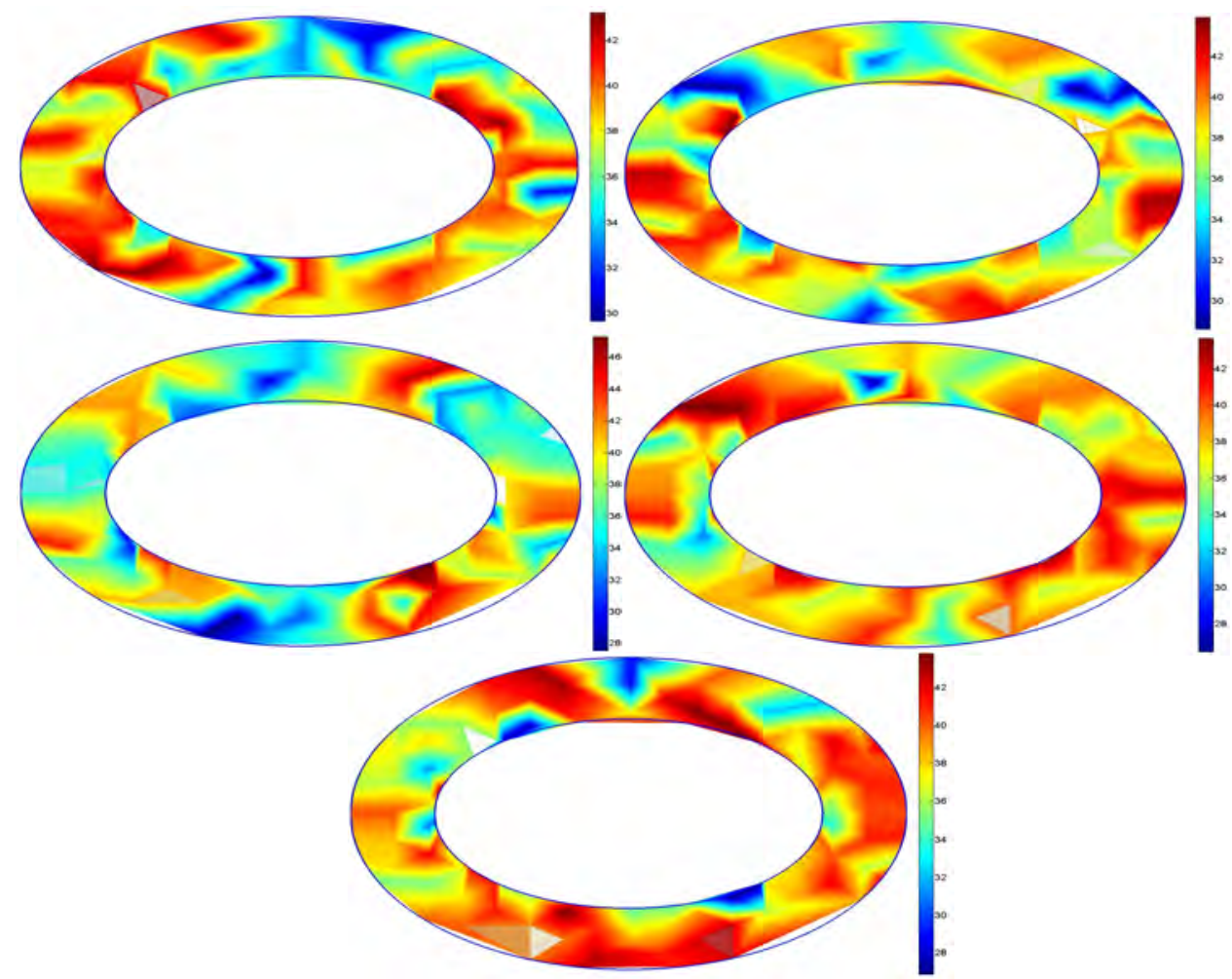

Figura 3.5: Asignación de propiedades mecánicas en una geometría elíptica utilizando el algoritmo desarrollado

En la Figura 3.6 se observa un diagrama de flujo que explica el funcionamiento del algoritmo.

\subsection{Selección del modelo numérico}

En el capítulo 2 se presentaron los modelos propuestos para analizar el proceso de fisuración en estructuras de hormigón. En esta sección se realiza 


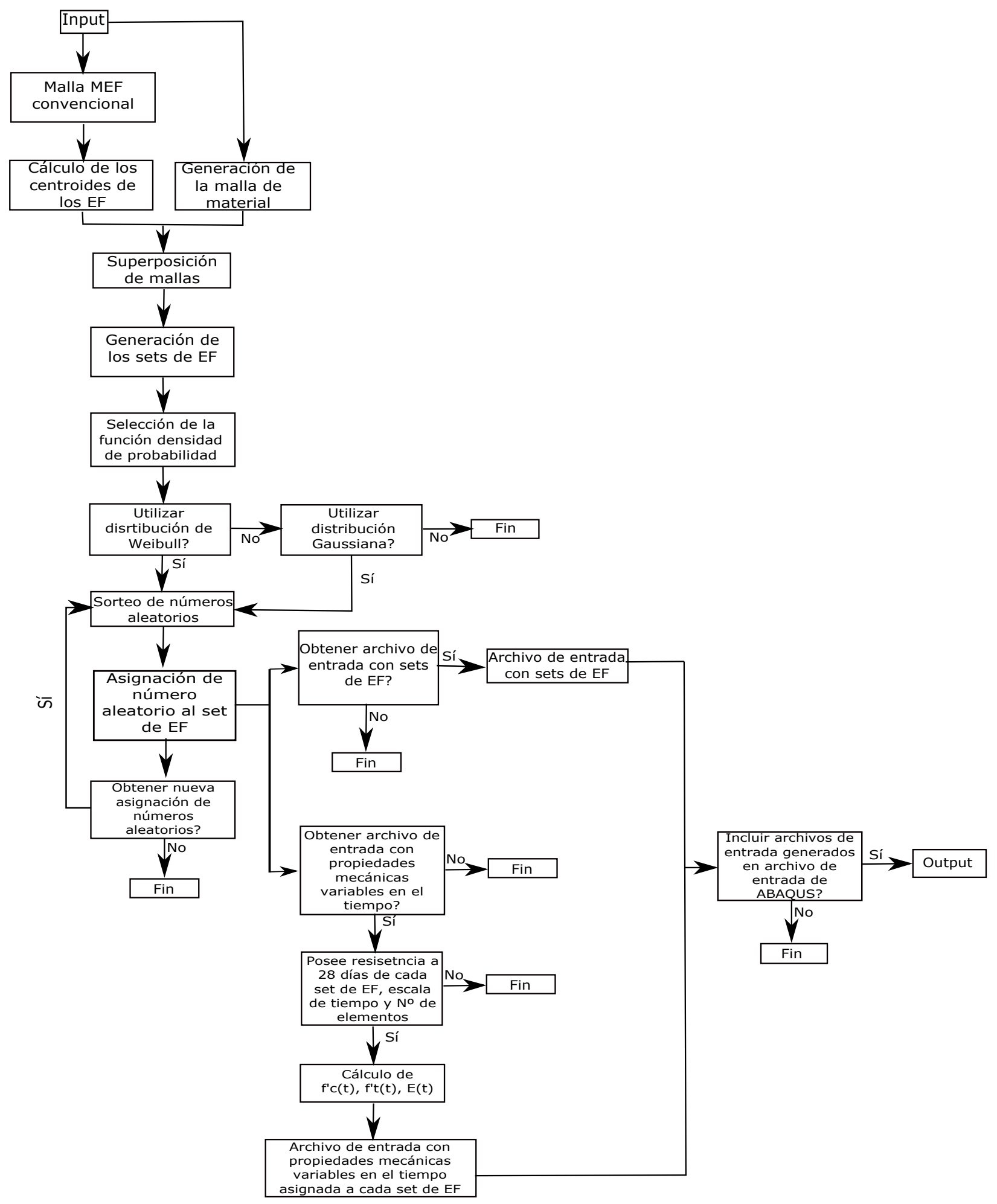

Figura 3.6: Diagrama de flujo que describe el funcionamiento del algoritmo desarrollado 
CAPítulo 3. Algoritmo desarrollado y modelo numérico aplicado para el estudio del proceso de fisuración en estructuras de hormigón

un análisis comparativo entre los modelos Elementos cohesivos (EC), Modelo de plasticidad con daño y Método de los Elementos Finitos Extendido (XFEM).

Para ello se analizó un bloque de hormigón rectangular de $6 \mathrm{~m}$ de ancho y $2 \mathrm{~m}$ de alto empotrado en su base. El estado de cargas corresponde a una variación de temperatura igual a $-40^{\circ} \mathrm{C}$, la cual representa una contracción térmica uniforme. El análisis se realizó en dos dimensiones con una duración de 5 días.

Las propiedades mecánicas del hormigón se detallan en la Tabla 4.1. $f_{c 28}$ es la resistencia a compresión a 28 días, $f_{c t 28}$ es la resistencia a tracción a 28 días, $E_{28}$ es el módulo de elasticidad a 28 días, $\alpha$ es el coeficiente de expansión térmica, $\nu$ es el coeficiente de Poisson, $\delta$ es la densidad del hormigón, $w_{c}$ es la apertura crítica.

\begin{tabular}{|l|l|}
\hline \multicolumn{2}{|l|}{ Propiedades Mecánicas del hormigón } \\
\hline$f_{c 28}$ & $38 \mathrm{MPa}$ \\
\hline$f_{c t 28}$ & $2.9 \mathrm{MPa}$ \\
\hline$E_{28}$ & $34000 \mathrm{MPa}$ \\
\hline$\alpha$ & $1 \mathrm{e}-5 \frac{1}{{ }^{\circ} \mathrm{C}}$ \\
\hline$\nu$ & 0.2 \\
\hline$\delta$ & $2.45 \mathrm{e}-5 \mathrm{Kg} / \mathrm{m}^{3}$ \\
\hline$w_{c}$ & $0.0655 \mathrm{~mm}$ \\
\hline
\end{tabular}

Tabla 3.1: Propiedades mecánicas del hormigón utilizado en el análisis comparativo de los diferentes modelos numéricos para el estudio del proceso de fisuración

A los efectos de realizar el análisis comparativo entre los modelos se consideró que las propiedades mecánicas del hormigón no varían en el tiempo y se distribuyen de forma homogénea en la geometría analizada.

A continuación se presentarán los resultados obtenidos con cada uno de los modelos mencionados.

\section{Modelo de Fisura Cohesiva-Elementos cohesivos}

Existen distintas maneras de implementar numéricamente el Modelo de Fisura Cohesiva. El código ABAQUS (2013c) propone la utilización de elementos cohesivos y superficies cohesivas.

La conectividad de los elementos cohesivos es igual a la de los elementos convencionales, pero se los suele considerar constituidos por dos caras separadas por un espesor. El desplazamiento relativo de la cara inferior respecto de la cara superior medido a lo largo de la dirección del espesor representa la apertura o cierre de la interfase. El cambio relativo en la posición de las caras inferior y superior medido en el plano ortogonal al espesor cuantifica 
la tensión de corte en el elemento cohesivo. En la Figura 3.7 se observa la representación del elemento cohesivo, ABAQUS (2013c).

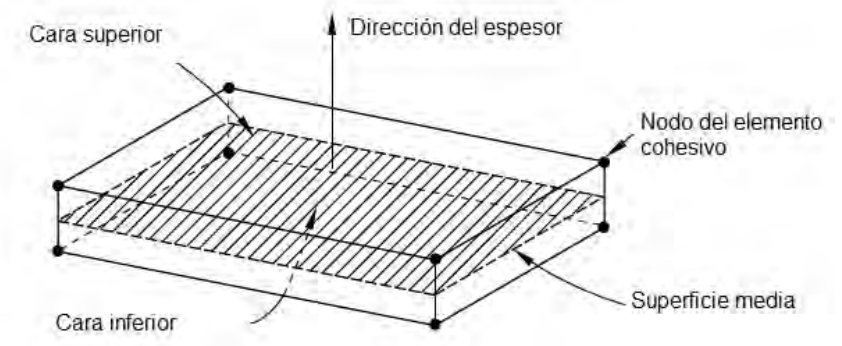

Figura 3.7: Representación de un elemento cohesivo, ABAQUS (2013c)

Las superficies cohesivas se diferencian de los elementos cohesivos en que los efectos del espesor de la interfase no son considerados, ya que en el caso de elementos cohesivos con respuesta tracción-separación, los efectos del espesor pueden incorporarse especificando un espesor distinto de cero para la interfase o calculándolo a partir de las coordenadas nodales del elemento cohesivo, ABAQUS (2013d).

ABAQUS (2013c) considera que el comportamiento previo al inicio del daño es elástico lineal, por lo cual existe una matriz constitutiva elástica que relaciona las tensiones normales y tangenciales con las aperturas del elemento fisurado en las direcciones normales y tangenciales, como puede verse en la siguiente expresión:

$$
\mathbf{t}=\left[\begin{array}{c}
t_{n} \\
t_{s} \\
t_{t}
\end{array}\right]=\left[\begin{array}{lll}
K_{n n} & K_{n s} & K_{n t} \\
K_{s n} & K_{s s} & K_{s t} \\
K_{t n} & K_{t s} & K_{t t}
\end{array}\right]\left[\begin{array}{l}
\varepsilon_{n} \\
\varepsilon_{s} \\
\varepsilon_{t}
\end{array}\right]=\mathbf{K} \varepsilon
$$

donde $\varepsilon_{n}=\frac{\delta_{n}}{T_{0}}, \varepsilon_{s}=\frac{\delta_{s}}{T_{0}}$ y $\varepsilon_{t}=\frac{\delta_{t}}{T_{0}}$.

En la expresión 3.5, $t$ es el vector de tracción nominal, cuya componente en dirección normal es $t_{n}$ y sus componentes en direcciones tangenciales son $t_{s} \mathrm{y} t_{t}$. Por otro lado, $\varepsilon_{n}, \varepsilon_{s}$ y $\varepsilon_{t}$ son las deformaciones correspondientes $\mathrm{y}$ $\delta_{n}, \delta_{s}$ y $\delta_{t}$ las aperturas en direcciones normal y tangencial, respectivamente. Finalmente $T_{0}$ representa el espesor del elemento cohesivo.

La matriz constitutiva elástica puede ser acoplada, es decir,tendrá todos sus elementos o desacoplada.

Tanto en las superficies cohesivas como en los elementos cohesivos basados en el comportamiento tracción-separación utilizados en la presente Tesis, debe definirse un criterio de inicio y un criterio de evolución de daño. Se proponen los siguientes criterios de inicio de daño, ABAQUS (2013c) : 
CAPítulo 3. Algoritmo desarrollado y modelo numérico aplicado para el estudio del proceso de fisuración en estructuras de hormigón

Criterio de la máxima tensión nominal

$$
f=\max \left\{\frac{\left\langle t_{n}\right\rangle}{t_{n}^{0}}, \frac{t_{s}}{t_{s}^{0}}, \frac{t_{t}}{t_{t}^{0}}\right\}
$$

En este caso $t_{n}^{0}, t_{s}^{0}$ y $t_{t}^{0}$ representan los valores pico de las tensiones nominales y los paréntesis de Macaulay indican que un estado de tensión de compresión pura no inicia el daño.

* Criterio de la máxima deformación nominal

$$
f=\max \left\{\frac{\left\langle\varepsilon_{n}\right\rangle}{\varepsilon_{n}^{0}}, \frac{\varepsilon_{s}}{\varepsilon_{s}^{0}}, \frac{\varepsilon_{t}}{\varepsilon_{t}^{0}}\right\}
$$

Criterio cuadrático de la tensión nominal

$$
f=\left\{\frac{\left\langle t_{n}\right\rangle}{t_{n}^{0}}\right\}^{2}+\left\{\frac{t_{s}}{t_{s}^{0}}\right\}^{2}+\left\{\frac{t_{t}}{t_{t}^{0}}\right\}^{2}
$$

Criterio cuadrático de la deformación nominal

$$
f=\left\{\frac{\left\langle\varepsilon_{n}\right\rangle}{\varepsilon_{n}^{0}}\right\}^{2}+\left\{\frac{\varepsilon_{s}}{\varepsilon_{s}^{0}}\right\}^{2}+\left\{\frac{\varepsilon_{t}}{\varepsilon_{t}^{0}}\right\}^{2}
$$

En todos los casos se considera que el daño se inicia cuando $f$ alcanza el valor 1 .

En la Figura 3.8 se indican las direcciones referidas anteriormente.

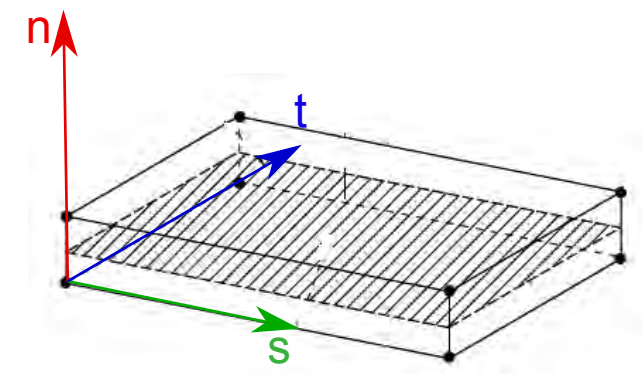

Figura 3.8: Direcciones de las tensiones nominales en un elemento cohesivo

En el caso de las superficies cohesivas los criterios de máxima deformación nominal y cuadrático de la deformación nominal se definen en términos de separación (aperturas) en lugar de deformaciones. 
Finalmente debe definirse una ley de evolución de daño, que puede ser lineal o no lineal, y describe la tasa a la cual la rigidez cohesiva se degrada una vez que se cumple con lo indicado en el criterio de inicio de daño. La variable de daño escalar $D$ representa el daño en la intersección entre la superficie de la fisura y el borde de los elementos fisurados. Al iniciarse el daño, $D$ evoluciona monotonamente desde 0 , su valor inicial, hasta 1 . Las tensiones normales y tangenciales son afectadas por el daño de acuerdo con las expresiones indicadas a continuación, ABAQUS (2013a):

$$
\begin{gathered}
t_{n}=\left\{\begin{array}{c}
(1-D) \bar{t}_{n} \quad \bar{t}_{n} \geq 0 \\
\bar{t}_{n} \quad \bar{t}_{n}<0
\end{array}\right. \\
t_{s}=(1-D) \bar{t}_{s} \\
t_{t}=(1-D) \bar{t}_{t}
\end{gathered}
$$

donde $\bar{t}_{n}, \bar{t}_{s} \mathrm{y} \bar{t}_{t}$ son las componentes normales y tangenciales predichas por el comportamiento elástico en tracción-separación para la separación actual sin daño.

La evolución del daño puede seguir una ley lineal, exponencial o puede ser definida en forma tabular.

Además se define una separación o apertura efectiva para describir la evolución en términos del comportamiento combinado de aperturas en dirección normal y tangencial, como se indica en 3.13:

$$
\delta_{m}=\sqrt{\left\{\left\langle\delta_{n}\right\rangle\right\}^{2}+\left\{\delta_{s}\right\}^{2}+\left\{\delta_{t}\right\}^{2}}
$$

\section{Aplicación del Modelo de Fisura Cohesiva}

Para el problema analizado, en primer lugar se determinó el tamaño máximo de elemento a utilizar en la malla de elementos finitos a partir de la expresión 3.14, Baẑant y Planas (1997):

$$
e=0,08 * \frac{E * G_{f}}{f_{c t}^{2}}
$$

donde $e$ es el tamaño de elemento, $E$ el módulo de elasticidad del material, $G_{f}$ la energía de fractura y $f_{c t}$ la resistencia a tracción. De la expresión 3.14 se obtiene que el tamaño máximo de elemento es $31 \mathrm{~mm}$ de lado, utilizando las propiedades del hormigón de la Tabla 3.1.

En el problema analizado no existe una fisura inicial. Por este motivo, se colocaron elementos cohesivos en todas las interfaces en dirección vertical, ya que por la naturaleza del fenómeno analizado las fisuras solo se localizan en dicha dirección. Se utilizaron 4958 elementos cohesivos (COH2D4) y 10117 
CaPítulo 3. Algoritmo desarrollado y modelo numérico aplicado para el estudio del proceso de fisuración en estructuras de hormigón

elementos cuadrados de cuatro nodos en tensión plana (CPS4), por lo que la malla está compuesta por 15075 elementos en total. En la Figura 3.9 se observa la malla de elementos finitos utilizada.

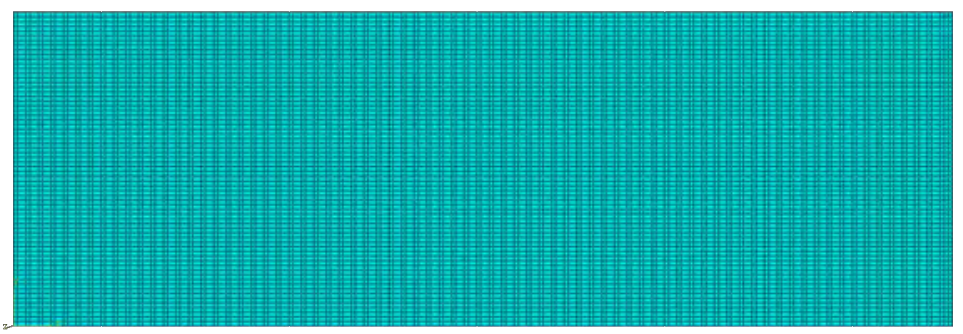

Figura 3.9: Malla de elementos finitos del bloque estudiado para la aplicación del Modelo de Fisura Cohesiva-Elementos cohesivos

El criterio de inicio de daño adoptado fue el determinado por la expresión 3.6 basado en las tensiones máximas. El valor de $t_{n}^{0}$ corresponde al de la resistencia a tracción a 28 días, indicado en la Tabla 3.1. El valor de $t_{s}^{0}$ se consideró igual al de $t_{n}^{0}$ y el de $t_{t}^{0}$ igual a 0 , ya que se está analizando un problema en dos dimensiones.

El criterio de evolución adoptado corresponde a una ley de ablandamiento lineal, especificando el valor del desplazamiento crítico, $w_{c}$, como se indica en la Tabla 3.1.

Aunque la flexibilidad de los elementos cohesivos puede contribuir a la deformación global de la estructura, su único propósito es simular el proceso de fisuración. Si la contribución del elemento cohesivo a la deformación global no es lo suficientemente pequeña comparada con la deformación volumétrica obtenida a partir de las ecuaciones constitutivas, no se asegura una conexión rígida entre los elementos finitos convencionales, vinculados entre si mediante elementos cohesivos, antes de que comience el proceso de fisuración. El valor de la flexibilidad queda determinado a partir de la rigidez $K$, sin embargo valores muy grandes de $K$ pueden causar problemas numéricos, como oscilaciones espurias de las tensiones. Por este motivo debe utilizarse un valor de $K$ lo suficientemente grande como para proveer una rigidez razonable y lo suficientemente pequeño como para no generar problemas numéricos, Turon et al. (2007). Se realizó un análisis a los efectos de optimizar el valor de la rigidez $K$ para el problema analizado, como resultado del mismo se adoptó un valor de $K$ igual a 100 veces el módulo de elasticidad del material.

Para estimar el tiempo de aparición de fisuras se determinó en los elementos cohesivos la variable SDEG, la cual varía entre 0 y 1 . El valor 1 representa a un elemento cohesivo completamente abierto, es decir, cuando la fisura deja de ser ficticia y pasa a representar una fisura real. Del patrón de fisuración obtenido, presentado en la Figura 3.10, se observa que en todas las zonas en las que se colocaron elementos cohesivos la variable SDEG 
alcanzó el valor 1, lo cual significa que en esas zonas aparecieron fisuras. Si en el problema analizado se coloca menor cantidad de elementos cohesivos se obtiene una menor cantidad de zonas fisuradas, ya que para este problema siempre se activan todos los elementos cohesivos. Esto se comprobó a través de distintas simulaciones. Por este motivo los resultados obtenidos, para el problema analizado, dependen de la cantidad de interfases en las que se encuentran presentes este tipo de elementos.

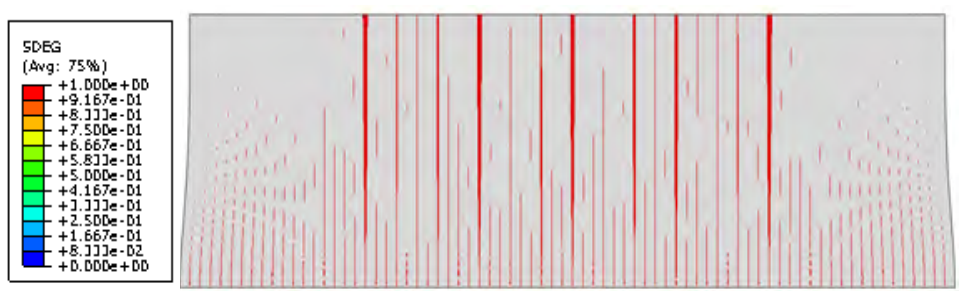

Figura 3.10: Patrón de fisuración obtenido aplicando elementos cohesivos para el bloque estudiado en el análisis comparativo de modelos numéricos

\section{Modelos de plasticidad con daño}

El código de análisis por elementos finitos Abaqus/Standard incorpora las ecuaciones de plasticidad con daño desarrolladas por Lubliner et al. (1989) considerando las modificaciones introducidas por Lee y Fenves (1998), en el modelo denominado Concrete Damaged Plasticity (CDP). Como su nombre lo indica, este es un modelo continuo de plasticidad con daño para hormigón, en donde se asume que los dos principales mecanismos de falla son fisuración en tracción y aplastamiento en compresión, ABAQUS (2013e). A continuación se presentan las ecuaciones que describen el modelo.

\section{Descomposición elasto-plástica de las deformaciones e incorpora- ción de la degradación de la rigidez (variable de daño)}

De acuerdo con la teoría incremental de la plasticidad, el tensor de deformaciones puede descomponerse en una parte elástica y en una parte plástica. Escribiendo esto en términos del tensor tasa de deformación: $(\dot{\varepsilon})$ se obtiene:

$$
\dot{\varepsilon}=\dot{\varepsilon}^{e l}+\dot{\varepsilon}^{p l}
$$

donde $\dot{\varepsilon}^{e l}$ es la parte elástica del tensor tasa de deformación y $\dot{\varepsilon}^{p l}$ es su parte plástica.

A su vez la relación tensión-deformación se encuentra gobernada por una variable de daño de tipo escalar, como se indica en 3.16:

$$
\sigma=(1-d) \mathbf{D}_{0}^{e l}:\left(\varepsilon-\varepsilon^{p l}\right)=\mathbf{D}^{e l}:\left(\varepsilon-\varepsilon^{p l}\right) \in\left\{\bar{\sigma} \mid F\left(\bar{\sigma}, \tilde{\varepsilon}^{p l}\right) \leq 0\right\}
$$


CaPítulo 3. Algoritmo desarrollado y modelo numérico aplicado para el estudio del proceso de fisuración en estructuras de hormigón

en la cual, $\mathbf{D}_{0}^{e l}$ es la rigidez elástica inicial del material ( $\sin$ daño), $\mathbf{D}^{e l}$ es la rigidez elástica degradada y $\boldsymbol{d}$ es la variable escalar de degradación de la rigidez o variable de daño, la cual puede tomar valores entre 0 (material sano) y 1 (material completamente dañado), ABAQUS (2013e).

El daño asociado a los mecanismos de falla del hormigón se corresponde con una reducción de la rigidez elástica. En el contexto de la teoría de daño escalar, la degradación de la rigidez es isótropa y se caracteriza por una única variable de degradación, $\boldsymbol{d}$. De acuerdo con la mecánica de daño en el continuo, la tensión efectiva se define como se indica en 3.17:

$$
\bar{\sigma}=\mathbf{D}_{0}^{e l}:\left(\varepsilon-\varepsilon^{p l}\right)
$$

El tensor de tensiones de Cauchy se relaciona con el tensor de tensiones efectivo a través de la variable de daño:

$$
\sigma=(1-d) \bar{\sigma}
$$

Para cualquier sección transversal del material, el factor $(1-d)$ representa la capacidad efectiva de tomar carga que tiene toda la sección. En ausencia de daño la tensión efectiva es igual a la tensión de Cauchy. Ante la aparición de daño la tensión efectiva es más representativa que la tensión de Cauchy porque es el área efectiva (área total menos área dañada) la que resiste las cargas externas, por lo que es conveniente formular el problema de plasticidad en términos de tensiones efectivas. La evolución de la variable de daño depende de las variables de endurecimiento y de la tensión efectiva, es decir $d=d\left(\bar{\sigma}, \tilde{\varepsilon}^{p l}\right)$, ABAQUS (2013e).

Los estados de daño en tracción y compresión se encuentran caracterizados independientemente por dos variables de endurecimento, $\tilde{\varepsilon}_{t}^{p l}$ y $\tilde{\varepsilon}_{c}^{p l}$, las cuales se denominan deformaciones plásticas equivalentes en tracción y compresión, respectivamente. La evolución de las variables de endurecimiento se determina a partir de la expresión 3.19:

$$
\dot{\tilde{\varepsilon}}^{p l}=\mathbf{h}\left(\sigma, \tilde{\varepsilon}^{p l}\right) \cdot \dot{\varepsilon}^{p l}
$$

donde

$$
\tilde{\varepsilon}^{p l}=\left[\begin{array}{c}
\tilde{\varepsilon}_{t}^{p l} \\
\tilde{\varepsilon}_{c}^{p l}
\end{array}\right]
$$

La microfisuración y el aplastamiento se representan mediante valores crecientes de las variables de endurecimiento, que controlan la evolución de la superficie de fluencia y la degradación de la rigidez elástica. Además, están íntimamente relacionadas con la disipación de la energía de fractura para generar microfisuras. 


\section{Criterio de plasticidad (Función de fluencia)}

La función de fluencia $F\left(\bar{\sigma}, \varepsilon^{\tilde{p} l}\right)$, representa una superficie en el espacio de las tensiones efectivas, e indica el nivel de tensiones para el cual se produce la falla. En un modelo no viscoso se cumple:

$$
F\left(\bar{\sigma}, \tilde{\varepsilon}^{p l}\right) \leq 0
$$

En este caso la función de fluencia está basada en la propuesta por Lubliner et al. (1989) e incorporando las modificaciones introducidas por Lee y Fenves (1998). Estas modificaciones permiten considerar la diferente evolución que presenta la resistencia bajo tracción y compresión, y se ponen de manifiesto a través de la dependencia del factor $\beta$ con la variable de endurecimiento. En 3.22 se indica la función de fluencia en términos de tensiones efectivas, ABAQUS (2013e):

$$
F\left(\bar{\sigma}, \tilde{\varepsilon}^{p l}\right)=\frac{1}{1-\alpha}\left(\bar{q}-3 \alpha \bar{p}+\beta\left(\tilde{\varepsilon}^{p l}\right)\left\langle\hat{\bar{\sigma}}_{\max }\right\rangle-\gamma\left\langle-\hat{\bar{\sigma}}_{\max }\right\rangle\right) \bar{\sigma}_{c}\left(\tilde{\varepsilon}_{c}^{p l}\right) \leq 0
$$

A continuación se describirán cada uno de los términos de la expresión 3.22 .

$\bar{p}$ representa la presión hidrostática efectiva y se expresa:

$$
\bar{p}=-\frac{1}{3} \bar{\sigma}: \mathbf{I}
$$

$\bar{q}$ es la tensión equivalente de Von Mises efectiva:

$$
\bar{q}=\sqrt{\frac{3}{2} \overline{\mathbf{S}}: \overline{\mathbf{S}}}
$$

$\overline{\mathbf{S}}$ es la parte desviadora del tensor de tensiones efectiva $\bar{\sigma}$, y se expresa:

$$
\overline{\mathbf{S}}=\bar{p} \mathbf{I}+\bar{\sigma}
$$

La función $\beta\left(\tilde{\varepsilon}^{p l}\right)$ se expresa como se indica en 3.26, Lee y Fenves (1998):

$$
\beta\left(\tilde{\varepsilon}^{p l}\right)=\frac{\bar{\sigma}_{c}\left(\tilde{\varepsilon}_{c}^{p l}\right)}{\bar{\sigma}_{t}\left(\tilde{\varepsilon}_{t}^{p l}\right)}(1-\alpha)-(1+\alpha)
$$

donde $\bar{\sigma}_{t}$ y $\bar{\sigma}_{c}$ son las tensiones de cohesión efectivas en tracción y compresión, respectivamente.

Por otro lado, $\hat{\bar{\sigma}}_{\max }$ es algebráicamente el máximo autovalor de $\bar{\sigma}$. En el caso de compresión biaxial $\hat{\bar{\sigma}}_{\max }=0$, por lo que la ecuación 3.22 se reduce a la función de fluencia de Drucker-Prager. El coeficiente $\alpha$ se determina a partir de las tensiones de fluencia en compresión equibiaxial y uniaxial inicial, indicadas como $\sigma_{b 0}$ y $\sigma_{c 0}$, respectivamente, como puede verse en 3.27, Lubliner et al. (1989):

$$
\alpha=\frac{\sigma_{b 0}-\sigma_{c 0}}{2 \sigma_{b 0}-\sigma_{c 0}}
$$


CAPítulo 3. Algoritmo desarrollado y modelo numérico aplicado para el estudio del proceso de fisuración en estructuras de hormigón

Los valores típicos de la relación $\frac{\sigma_{b 0}}{\sigma_{c 0}}$ para el hormigón están en el rango de 1.10 a 1.16 , lo cual conduce a valores de $\alpha$ comprendidos entre 0.08 y 0.12, Lubliner et al. (1989).

El coeficiente $\gamma$ se considera en la función de fluencia solo para estados de compresión triaxial, es decir, cuando $\hat{\bar{\sigma}}_{\max }<0$. Este coeficiente se determina comparando la condición de fluencia a lo largo de los meridianos de tracción y compresión. Por definición, el meridiano de tracción (TM) es el lugar de los estados tensionales que satisfacen la condición $\hat{\bar{\sigma}}_{\max }=\hat{\bar{\sigma}}_{1}>\hat{\bar{\sigma}}_{2}=\hat{\bar{\sigma}}_{3}$, y el meridiano de compresión (CM) es el lugar de los estados tensionales tales que $\hat{\bar{\sigma}}_{\text {max }}=\hat{\bar{\sigma}}_{1}=\hat{\bar{\sigma}}_{2}>\hat{\bar{\sigma}}_{3}$, donde $\hat{\bar{\sigma}}_{1}, \hat{\bar{\sigma}}_{2} \mathrm{y} \hat{\bar{\sigma}}_{3}$ son los autovalores del tensor de tensiones efectivas. Se puede demostrar que $(\hat{\bar{\sigma}})_{T M}=\frac{2}{3} \bar{q}-\bar{p}$ y $\hat{\bar{\sigma}}_{\max }=\frac{1}{3} \bar{q}-\bar{p}$, a lo largo de los meridianos de tracción y compresión, respectivamente. Para $\hat{\bar{\sigma}}_{\text {max }}<0$ las condiciones de rotura serán, Lubliner et al. (1989):

$$
\begin{aligned}
& \left(\frac{2}{3} \gamma+1\right) \bar{q}-(\gamma+3 \alpha) \bar{p}=(1-\alpha) \bar{\sigma}_{c} \\
& \left(\frac{1}{3} \gamma+1\right) \bar{q}-(\gamma+3 \alpha) \bar{p}=(1-\alpha) \bar{\sigma}_{c}
\end{aligned}
$$

Si se define $\mathbf{K}_{c}=\frac{\bar{q}_{(T M)}}{\bar{q}_{(C M)}}$ para cualquier valor dado de la presión hidrostática $\bar{p}$ con $\hat{\bar{\sigma}}_{\max }<0$, se obtiene:

$$
\mathbf{K}_{c}=\frac{\gamma+3}{2 \gamma+3}
$$

El hecho de que $\mathbf{K}_{c}$ sea constante no se contradice con la evidencia experimental, Lubliner et al. (1989). El coeficiente $\gamma$ se obtiene a partir de la expresión 3.31, como se indica a continuación:

$$
\gamma=\frac{3\left(1-\mathbf{K}_{c}\right)}{2 \mathbf{K}_{c}-1}
$$

Típicamente para hormigón se utiliza $\mathbf{K}_{c}=\frac{2}{3}$, lo cual da lugar a $\gamma=3$, Lubliner et al. (1989).

En el caso de que $\hat{\bar{\sigma}}_{\max }>0$, la condición de rotura a lo largo de los meridianos de tracción y compresión se reduce a:

$$
\begin{array}{ll}
\left(\frac{2}{3} \beta+1\right) \bar{q}-(\beta+3 \alpha) \bar{p}=(1-\alpha) \bar{\sigma}_{c} \quad(T M) \\
\left(\frac{1}{3} \beta+1\right) \bar{q}-(\beta+3 \alpha) \bar{p}=(1-\alpha) \bar{\sigma}_{c} \quad(C M)
\end{array}
$$

Si para este caso se define $\mathbf{K}_{c}=\frac{\bar{q}_{(T M)}}{\bar{q}_{(C M)}}$ para cualquier valor dado de la presión hidrostática $\bar{p}$ con $\hat{\bar{\sigma}}_{\max }>0$, se obtiene:

$$
\mathbf{K}_{t}=\frac{\beta+3}{2 \beta+3}
$$


En la Figura 3.11 se muestra la superficie de fluencia típica en el plano desviador y en la Figura 3.12 la superficie de fluencia para la condición de tensión plana.

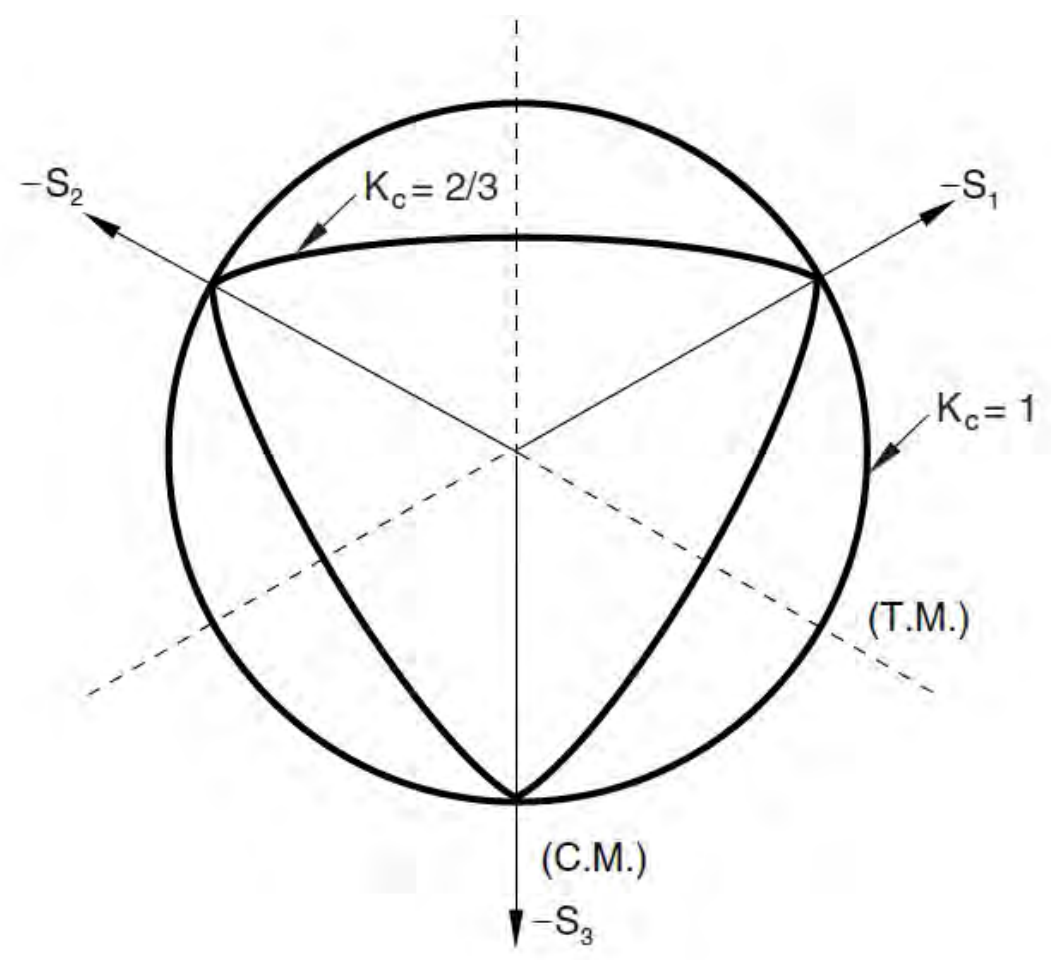

Figura 3.11: Superficie de fluencia en el plano desviador correspondiente a diferentes valores de $\mathbf{K}_{c}$, ABAQUS (2013b)

\section{Regla de flujo}

La regla de flujo determina la dirección de la deformación plástica de acuerdo con la siguiente expresión:

$$
\dot{\varepsilon}^{p l}=\dot{\lambda} \frac{\partial G(\bar{\sigma})}{\partial \bar{\sigma}}
$$

donde, $\lambda$ es una constante denominada multiplicador plástico que determina la cantidad de deformación plástica y $\boldsymbol{G}$ es una función de las tensiones efectivas, denominada potencial plástico. El gradiente de $\boldsymbol{G}$ determina la dirección de la deformación plástica.

Se sabe que los materiales granulares, como el hormigón, presentan un cambio significativo de volumen cuando son sometidos a estados inelásticos severos. Este cambio de volumen, causado por distorsión plástica, puede 
CAPítulo 3. Algoritmo desarrollado y modelo numérico aplicado para el estudio del proceso de fisuración en estructuras de hormigón

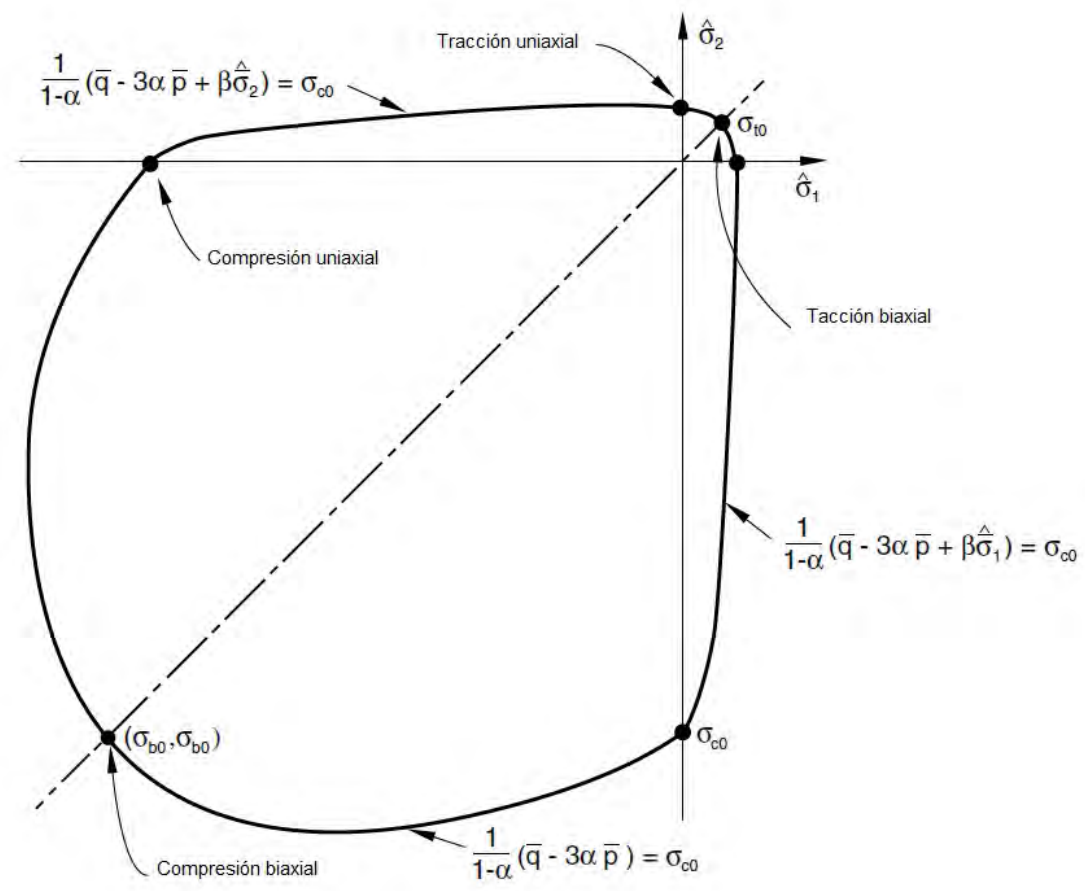

Figura 3.12: Superficie de fluencia en tensión plana, ABAQUS (2013b)

reproducirse correctamente utilizando una función de potencial plástico $(\mathbf{G})$ adecuada en la definición de la regla de flujo, ecuación 3.35, Lubliner et al. (1989). Como en este modelo la función de fluencia es diferente a la función del potencial plástico, la regla de flujo se denomina no asociada.

El potencial plástico elegido en el modelo CDP es la función hiperbólica de Drucker-Prager, Drucker y Prager (1952); ABAQUS (2013e), la cual se indica a continuación:

$$
G=\sqrt{\left(\epsilon \sigma_{t 0} \tan \psi\right)^{2}+\bar{q}^{2}}-\bar{p} \tan \psi
$$

En la ecuación anterior $\psi$ es el ángulo de dilatación medido en el plano $p$ - $q$ para altas presiones de confinamiento, $\sigma_{t 0}$ es la tensión uniaxial de tracción a rotura y $\epsilon$ es un parámetro denominado excentricidad, el cual define la tasa a la cual la función alcanza la asíntota (el potencial plástico tiende a una línea recta si la excentricidad tiende a cero). El potencial plástico es una función continua y suave que asegura que la dirección del flujo sea única. Dicha función alcanza asintóticamente el potencial plástico lineal de Drucker-Prager para altas presiones de confinamiento e intersecta el eje de presión hidrostática a 90 , ABAQUS (2013e).

Al tratarse de un potencial plástico no asociado, el uso de un modelo 
de daño plástico en hormigón requiere de la resolución de ecuaciones no simétricas.

En base a lo descripto hasta ahora, puede concluirse que la respuesta elasto-plástica de este modelo de plasticidad con daño se describe en términos de la tensión efectiva y de las variables de endurecimiento.

Debe indicarse que $\dot{\lambda}$ y $\mathbf{F}$ cumplen con la condición de Kuhn-Tucker: $\dot{\lambda} F=0 ; \dot{\lambda} \geq 0 ; f \leq 0$.

Es importante mencionar que las relaciones constitutivas de la respuesta elasto-plástica, ecuaciones $3.16,3.21$ y 3.35, están desacopladas con respecto a la degradación de la rigidez (daño), indicada en la ecuación 3.18, lo cual hace que el modelo sea atractivo por presentar una implementación numérica efectiva.

\section{Daño y degradación de la rigidez}

Se asume que las curvas tensión-deformación uniaxiales pueden transformarse en curvas tensión-deformación plástica de la forma que se indica a continuación:

$$
\begin{aligned}
\sigma_{t} & =\sigma\left(\tilde{\varepsilon}_{t}^{p l}, \dot{\tilde{\varepsilon}}_{t}^{p l}, \theta, f_{i}\right) \\
\sigma_{c} & =\sigma\left(\tilde{\varepsilon}_{c}^{p l}, \dot{\tilde{\varepsilon}}_{c}^{p l}, \theta, f_{i}\right)
\end{aligned}
$$

Los subíndices $t$ y $c$ se refieren a tensión y compresión, respectivamente. Como se indicó anteriormente, $\dot{\vec{\varepsilon}}_{t}^{p l}$ y $\dot{\tilde{\varepsilon}}_{c}^{p l}$ son las tasas de deformación plástica equivalente, $\tilde{\varepsilon}_{t}^{p l}=\int_{0}^{t} \dot{\tilde{\varepsilon}}_{t}^{p l} d t$ y $\tilde{\varepsilon}_{c}^{p l}=\int_{0}^{c} \dot{\tilde{\varepsilon}}_{c}^{p l} d t$ son las deformaciones plásticas equivalentes, $\theta$ es la temperatura y $f_{i}(i=1,2, \ldots)$ son otras variables de campo predefinidas.

Bajo condiciones de carga uniaxial las tasas de deformación plástica efectiva vienen definidas como se indica a continuación:

$$
\begin{aligned}
& \dot{\tilde{\varepsilon}}_{t}^{p l}=\dot{\varepsilon}_{11}^{p l} \\
& \dot{\tilde{\varepsilon}}_{c}^{p l}=-\dot{\varepsilon}_{11}^{p l}
\end{aligned}
$$

Se considerará por convención que $\sigma_{c}$ es una cantidad positiva que representa la magnitud de la tensión uniaxial de compresión, $\sigma_{c}=-\sigma_{11}$, ABAQUS (2013e). Como puede verse en la Figura 3.13 cuando la probeta de hormigón se descarga desde cualquier punto de la rama de ablandamiento de la curva tensión deformación, la respuesta en descarga se observa debilitada, ya que la rigidez elástica del material parece estar dañada o degradada. La degradación de la rigidez elástica es significativamente diferente en tracción y compresión, siendo este efecto cada vez más pronunciado a medida que la deformación plástica se incrementa. La respuesta degradada del hormigón se caracteriza por dos variables de daño uniaxiales independientes, $d_{t} \mathrm{y}$ $d_{c}$, las cuales son funciones de la deformación plástica, la temperatura y las variables de campo. Las variables de daño uniaxial son funciones crecientes 
CAPítulo 3. Algoritmo desarrollado y modelo numérico aplicado para el estudio del proceso de fisuración en estructuras de hormigón
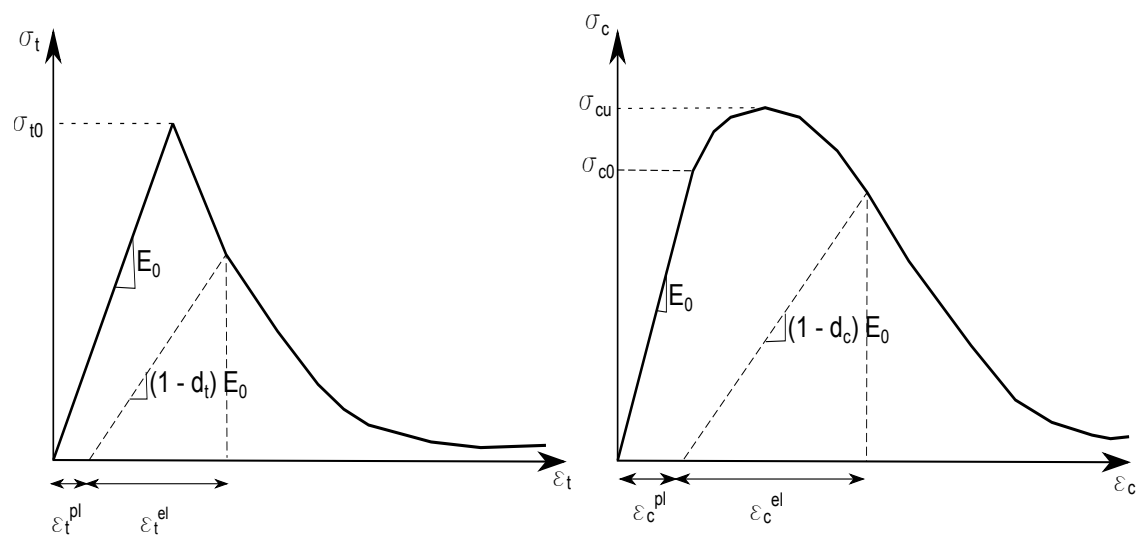

Figura 3.13: Respuesta del hormigón bajo carga uniaxial en tracción (a) y compresión (b), ABAQUS (2013b)

de la deformación plástica equivalente. Pueden tomar valores entre 0 , para material sin daño, a 1, para material completamente dañado.

Si $E_{0}$ es la rigidez elástica inicial del material, las relaciones tensióndeformación bajo cargas uniaxiales de tracción y compresión son las que se indican a continuación:

$$
\begin{aligned}
& \sigma_{t}=\left(1-d_{t}\right) E_{0}\left(\varepsilon_{t}-\tilde{\varepsilon}_{t}^{p l}\right) \\
& \sigma_{c}=\left(1-d_{c}\right) E_{0}\left(\varepsilon_{c}-\tilde{\varepsilon}_{c}^{p l}\right)
\end{aligned}
$$

Bajo cargas uniaxiales las fisuras se propagan en dirección transversal a la dirección de las tensiones. Por lo tanto la nucleación y propagación de fisuras causa una reducción del área disponible para tomar carga, lo cual conduce a un incremento de las tensiones efectivas. El efecto es menos pronunciado bajo cargas de compresión ya que las fisuras corren paralelas a la dirección de carga; sin embargo, después de una cantidad significativa de aplastamiento, el área efectiva de capacidad de carga también se reduce significativamente. Las tensiones uniaxiales efectivas de cohesión, $\bar{\sigma}_{t} \mathrm{y} \bar{\sigma}_{c}$ vienen dadas por las siguientes expresiones:

$$
\begin{aligned}
& \bar{\sigma}_{t}=\frac{\sigma_{t}}{\left(1-d_{t}\right)}=E_{0}\left(\varepsilon_{t}-\tilde{\varepsilon}_{t}^{p l}\right) \\
& \bar{\sigma}_{c}=\frac{\sigma_{c}}{\left(1-d_{c}\right)}=E_{0}\left(\varepsilon_{c}-\tilde{\varepsilon}_{c}^{p l}\right)
\end{aligned}
$$

La tensión de cohesión uniaxial efectiva determina el tamaño de la superficie de fluencia.

\section{Definición del endurecimiento en tracción}

El endurecimiento en tracción puede especificarse mediante una relación tensión-deformación post-falla o aplicando un criterio de fisuración basado en la energía de fractura. 
El primer criterio se aplica al hormigón armado. En ese caso la especificación del comportamiento post-falla implica indicar la tensión post-falla como función de la deformación por fisuración, $\tilde{\varepsilon}_{t}^{c k}$. La deformación por fisuración se define como la deformación total menos la deformación elástica correspondiente al material intacto, ver Figura 3.14.

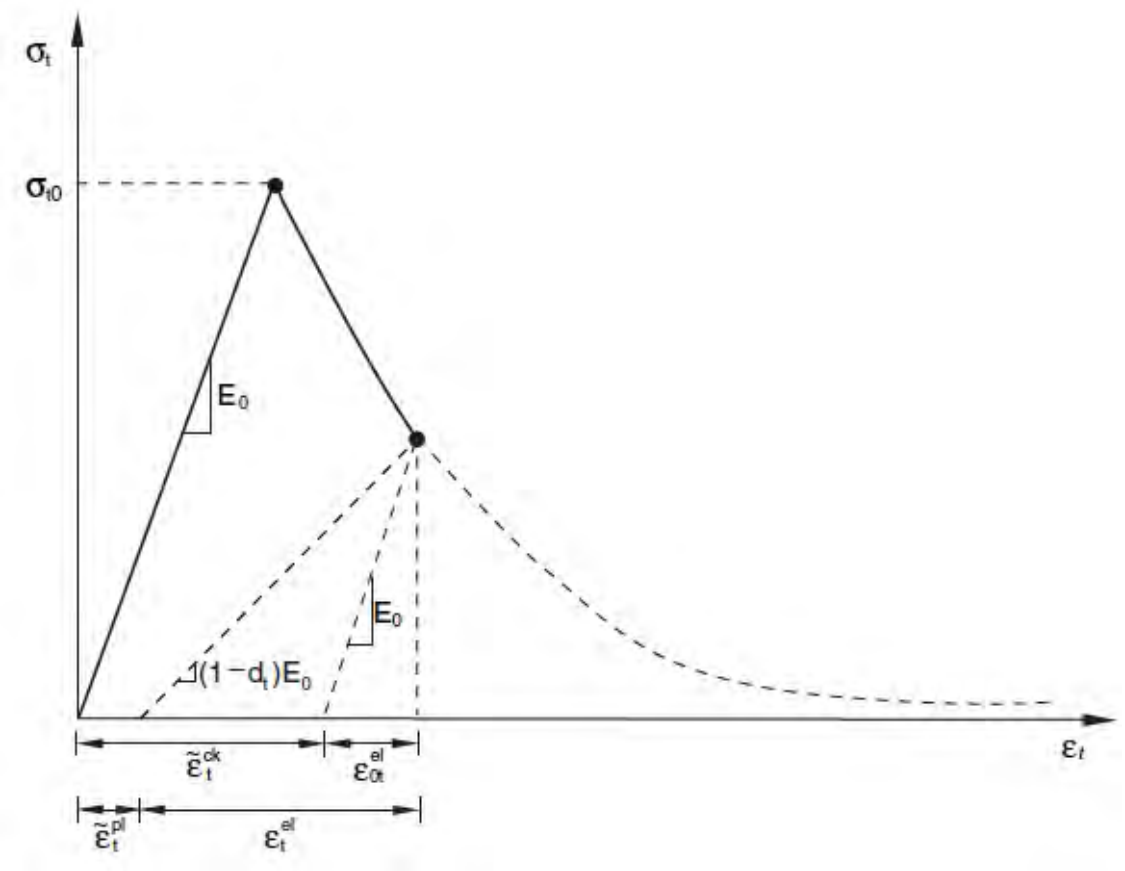

Figura 3.14: Definición de la deformación por fisuración utilizada para definir el endurecimiento en tracción, ABAQUS (2013b)

El otro criterio para definir el endurecimiento en tracción está basado en la energía de fractura y parece más adecuado para el caso del hormigón simple, ya que al no haber refuerzo el criterio descrito anteriormente en base a deformaciones genera sensibilidad de malla en los resultados, ABAQUS (2013b). Sin embargo, se acepta de manera general que la propuesta de la energía de fractura realizada por Hillerborg et al. (1976) es adecuada para mitigar este problema. Con esta aproximación, el comportamiento cuasi-frágil del hormigón se caracteriza mediante una respuesta tensión-desplazamiento en lugar de una respuesta tensión-deformación.

Este modelo de fisuración basado en energía de fractura se define especificando la tensión en función del desplazamiento en el momento de la fisuración. También puede definirse directamente a partir de la especificación de la energía de fractura como una propiedad del material. La tensión de rotura se especifica como una función de la energía de fractura, asumiendo una pérdida lineal de resistencia luego de la fisuración. 
CAPítulo 3. Algoritmo desarrollado y modelo numérico aplicado para el estudio del proceso de fisuración en estructuras de hormigón

En este caso se calcula un "desplazamiento plástico" en lugar de una deformación plástica, como se indica en 3.41, ABAQUS (2013b):

$$
w_{t}^{p l}=w_{t}^{c k}-\frac{d_{t}}{\left(1-d_{t}\right)} \frac{\sigma_{t} l_{0}}{E_{0}}
$$

donde $l_{0}$ representa la longitud del especimen.

La implementación del concepto tensión-desplazamiento en un modelo de elementos finitos requiere la definición de una longitud característica asociada con un punto de integración. La longitud característica de fisuración está basada en la geometría y formulación del elemento, ABAQUS (2013b).

\section{Definición del endurecimiento en compresión}

Las tensiones de compresión se definen como una función de la deformación inelástica, $\tilde{\varepsilon}_{c}^{i n}$, la tasa de deformación, la temperatura y otras variables de campo.

El endurecimiento se determina en términos de la deformación inelástica, la cual se define como la deformación total menos la deformación correspondiente al material intacto, como se indica a continuación y en la Figura 3.15:

$$
\tilde{\varepsilon}_{c}^{i n}=\varepsilon_{v}-\varepsilon_{0 c}^{e l}
$$

donde $\varepsilon_{0 c}^{e l}=\frac{\sigma_{c}}{E_{0}}$.

Las deformaciones plásticas en compresión se determinan a partir de la siguiente expresión:

$$
\tilde{\varepsilon}_{c}^{p l}=\tilde{\varepsilon}_{c}^{i n}-\frac{d_{c}}{\left(1-d_{c}\right)} \frac{\sigma_{c}}{E_{0}}
$$

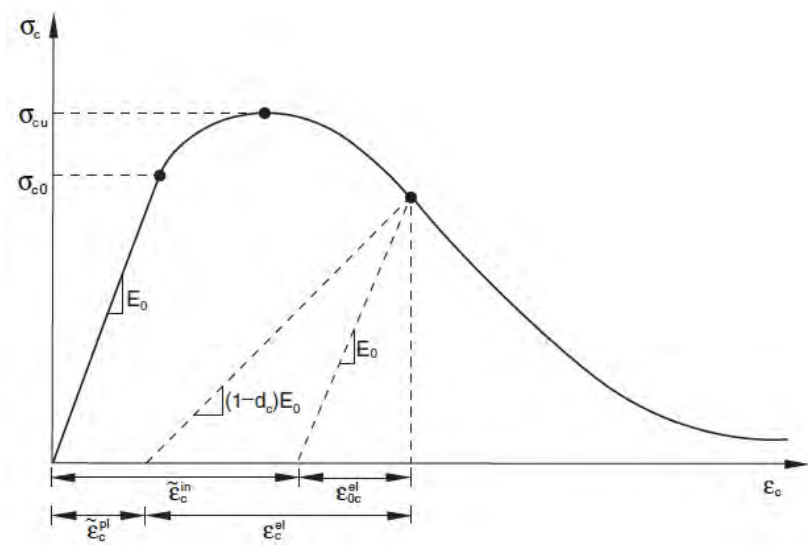

Figura 3.15: Definición de la deformación inelástica en compresión usada para el cálculo del endurecimiento en compresión 


\section{Regularización viscoplástica}

Los modelos de comportamiento de materiales que presentan ablandamiento y degradación de rigidez a menudo tienen severos problemas de convergencia en programas de análisis implícito. Algunas de estas dificultades pueden resolverse utilizando regularización viscoplástica de las ecuaciones constitutivas. En el caso del modelo analizado esta regularización viscoplástica permite que las tensiones se encuentren por fuera de la superficie de fluencia. Se utiliza una generalización de la regularización de Duvaut-Lions, de acuerdo con la cual el tensor tasa de deformación, $\dot{\varepsilon}_{v}^{p l}$, se define, ABAQUS (2013e):

$$
\dot{\varepsilon}_{v}^{p l}=\frac{1}{\mu}\left(\varepsilon^{p l}-\varepsilon_{v}^{p l}\right)
$$

en la cual, $\mu$ es el parámetro viscoso que representa la relajación en el tiempo del sistema viscoplástico y $\varepsilon^{p l}$ es la deformación plástica evaluada en el modelo no viscoso.

También debe definirse una degradación viscoplástica de la rigidez, como se indica en 3.45 :

$$
d_{v}=\frac{1}{\mu}\left(d-d_{v}\right)
$$

En este caso $d$ es la variable de degradación evaluada en el modelo no viscoso. La relación tensión-deformación del modelo viscoplástico se define como se indica a continuación:

$$
\sigma=\left(1-d_{v}\right) \mathbf{D}_{0}^{e l}:\left(\varepsilon-\varepsilon_{v}^{p l}\right)
$$

La solución del sistema viscoplástico relaja a la solución del sistema no viscoso tal que $t / \mu \rightarrow \infty$, donde $t$ representa el tiempo. La utilización de la regularización viscoplástica con un valor del parámetro de viscosidad pequeño, en comparación al incremento de tiempo característico, generalmente ayuda a mejorar la tasa de convergencia del modelo en el régimen de ablandamiento sin comprometer los resultados, ABAQUS (2013e).

\section{Aplicación del modelo de plasticidad con daño}

En el problema analizado las fisuras se originan exclusivamente por tensiones de tracción, por lo que se consideró que en compresión el material se comporta en régimen elástico. Para el comportamiento en tracción se adoptó una ley tensión-desplazamiento de tipo lineal. Además el modelo requiere la adopción de una ley daño-desplazamiento, que en este caso también se consideró de tipo lineal.

En la Tabla 3.2 se indican los parámetros del modelo de plasticidad con daño

Como se mencionó anteriormente, $\omega$ representa el ángulo de fricción interna, $\epsilon$ es la excentricidad, es decir, la tasa a la cual la función de flujo 
CAPítulo 3. Algoritmo desarrollado y modelo numérico aplicado para el estudio del proceso de fisuración en estructuras de hormigón

\begin{tabular}{|c|c|}
\hline \multicolumn{2}{|c|}{ Parámetros del modelo de plasticidad con daño } \\
\hline$\omega$ & $35^{\circ}$ \\
\hline$\epsilon$ & 1 \\
\hline$\sigma_{b 0} / \sigma_{c}$ & 1.2 \\
\hline$K$ & 0.67 \\
\hline
\end{tabular}

Tabla 3.2: Parámetros del modelo de plasticidad con daño utilizado en el análisis comparativo de los diferentes modelos numéricos para el estudio del proceso de fisuración

plástico alcanza la asíntota, $\sigma_{b 0} / \sigma_{c}$ es la relación entre la tracción y la compresión biaxial y $K$ es un factor que representa la relación entre el segundo invariante de la tensión en el meridiano de tracción respecto al meridiano de compresión en la fluencia inicial, para cualquier valor del invariante de presión.

En la Figura 3.16 se presenta la malla de elementos finitos utilizada.

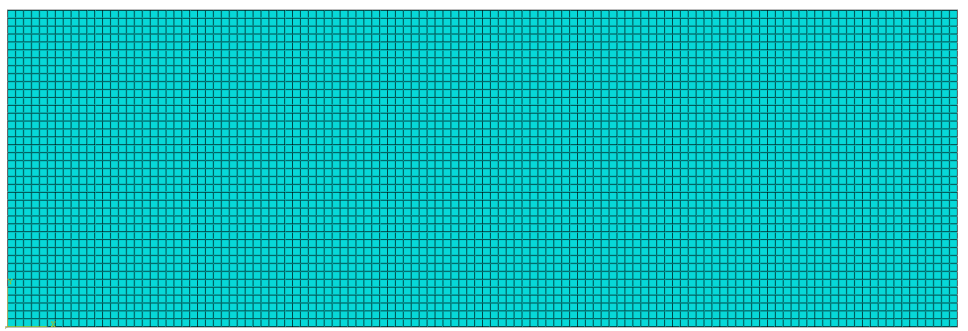

Figura 3.16: Malla de elementos finitos del bloque modelado para el análisis comparativo de los modelos numéricos aplicados al estudio del proceso de fisuración en hormigón

El bloque se discretizó utilizando 4800 elementos tipo CPS4 (elementos cuadriláteros de cuatro nodos en tensión plana). Para mejorar las condiciones de convergencia se adoptó un factor de amortiguamiento igual a $2 E^{-8}$, por lo que se verificó que la disipación de energía viscosa fuera menor al $10 \%$ de la energía interna del modelo, ABAQUS (2013b).

Se ha demostrado que este modelo de plasticidad con daño es dependiente del tamaño de la malla, Chaudhar y Chakrabart (2012), por lo que se realizó un análisis de sensibilidad para evitar la influencia del tamaño de los elementos en los resultados. Se consideraron dos tipos de elementos, cuadrados y triangulares, y tamaños de $150 \mathrm{~mm}, 100 \mathrm{~mm}, 50 \mathrm{~mm}, 40 \mathrm{~mm}, 25 \mathrm{~mm}$ y $12.5 \mathrm{~mm}$ de lado. En las Tablas 3.3 y 3.4 se indican los tiempos de aparición de la fisura para el mallado cuadrado y triangular, respectivamente, para cada una de las longitudes de lado de los elementos analizadas.

Como puede observarse en la Tabla 3.3, a partir de la utilización de 


\begin{tabular}{|c|c|}
\hline Longitud del lado del elemento [mm] & Tiempo de aparición fisuras [hs] \\
\hline 150 & 45.6 \\
\hline 100 & 42.24 \\
\hline 50 & 32.16 \\
\hline 40 & 31.94 \\
\hline 25 & 32.4 \\
\hline 12.5 & 32.22 \\
\hline
\end{tabular}

Tabla 3.3: Modelo de plasticidad con daño considerando un mallado cuadrado: tiempo de aparición de las fisuras

\begin{tabular}{|c|c|}
\hline Longitud del lado del elemento [mm] & Tiempo de aparición fisuras [hs] \\
\hline 150 & 39.72 \\
\hline 100 & 38.89 \\
\hline 50 & 35.39 \\
\hline 40 & 35.22 \\
\hline 25 & 35.28 \\
\hline 12.5 & 35.55 \\
\hline
\end{tabular}

Tabla 3.4: Modelo de plasticidad con daño considerando un mallado triangular: tiempo de aparición de las fisuras

elementos de $50 \mathrm{~mm}$ de lado no se observan variaciones considerables en los resultados. Por este motivo la longitud de lado de los elementos utilizados en el análisis fue de $50 \mathrm{~mm}$. Se decidió adoptar un mallado cuadrado para realizar la comparación de los modelos numéricos empleados para estudiar fisuración en el hormigón.

Por tratarse de un modelo continuo no tiene incorporado el concepto de desarrollo de fisuras en el punto de integración material, sin embargo es posible introducir el concepto de una dirección de fisuración efectiva con el objetivo de obtener una representación gráfica de los patrones de fisuración en la estructura, ABAQUS (2013b). De acuerdo con Lubliner et al. (1989), puede asumirse que el proceso de fisuración se inicia en los puntos en los que la deformación plástica equivalente en tracción es mayor a cero, y la máxima deformación principal plástica es positiva. La dirección del vector normal al plano de la fisura se asume paralela a la dirección de la máxima deformación principal plástica. Teniendo en cuenta lo anterior, en la Figura 3.17 se presentan los vectores representativos de la deformación principal máxima, los cuales tienen dirección normal a los planos de fisuración. En la Figura 3.18 pueden verse las zonas dañadas medidas mediante la variable de daño en tracción DAMAGET, la cual varía entre 0 y 1 , representando este último valor una zona completamente dañada.

A partir del análisis realizado se observa una zona dañada en el lado 
CAPítulo 3. Algoritmo desarrollado y modelo numérico aplicado para el estudio del proceso de fisuración en estructuras de hormigón
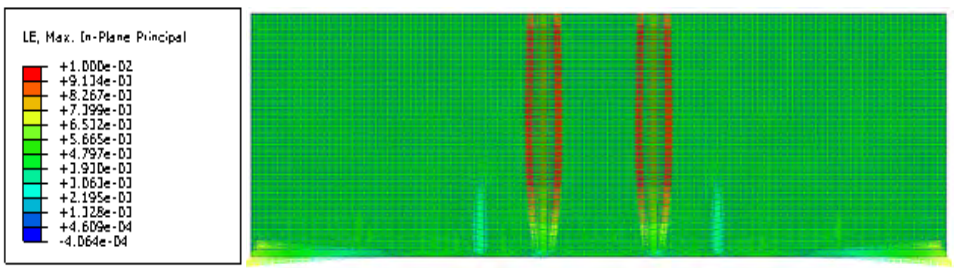

Figura 3.17: Vectores representativos de la deformación principal máxima para el bloque estudiado en el análisis comparativo de modelos utilizando el modelo CDP

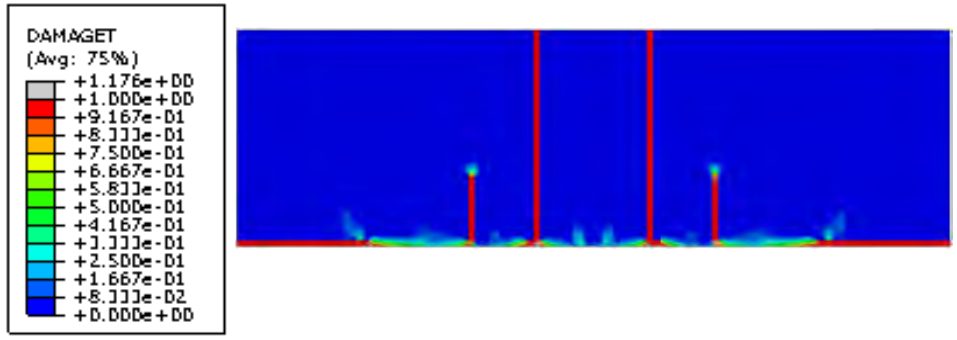

Figura 3.18: Representación de la variable de daño para el bloque estudiado en el análisis comparativo de modelos utilizando el modelo CDP

inferior del bloque, correspondiente con la ubicación del empotramiento, ya que la restricción impuesta por el mismo genera el desarrollo de tensiones altas que superan la resistencia a tracción. Se observa que esas zonas dañadas progresan hacia el interior del bloque, alcanzando el lado superior del mismo. Analizando el momento en que la deformación plástica equivalente en tracción (PEEQT) en relación al tiempo presenta un valor distinto de cero, se pudo determinar la aparición de una fisura a la edad de 32.16 horas.

\section{Métodos de los elementos finitos extendido (XFEM)}

En el capítulo 2 se presentaron los fundamentos del método XFEM, es la extensión del método de los elementos finitos tradicional basado en el método de partición de la unidad. A efectos de comparar distintos modelos para analizar el proceso de fisuración en un bloque de hormigón, se utilizó el modelo XFEM basado en el método de los segmentos cohesivos y nodos fantasma, a través de su implementación en el código comercial ABAQUS/Standard.

En la Tabla 3.1 se presentaron las propiedades mecánicas del material consideradas en el análisis.

La malla utilizada para la aplicación del método XFEM es la misma que se adoptó para el modelo CDP, presentada en la Figura 3.16.

Para la utilización del método XFEM es necesario definir un criterio de inicio de daño y un criterio de evolución, los cuales coinciden con los 
expuestos para elementos cohesivos en secciones anteriores, por lo que no volverán a enumerarse, solo se realizarán algunas aclaraciones.

En primer lugar, en el método XFEM las componentes de la matriz rigidez son desacopladas y se calculan en base a las propiedades elásticas del elemento enriquecido. A su vez, el vector de tracciones $\mathbf{t}$ se escribe $\mathbf{t}=K \delta$, es decir, en términos de aperturas o separaciones y no de deformaciones como en el caso de elementos cohesivos.

La segunda aclaración se vincula a los criterios de inicio de daño, ya que en el caso del método XFEM, se proponen dos criterios adicionales a los indicados para elementos cohesivos, los cuales se presentan a continuación, ABAQUS (2013a):

* Criterio de la tensión principal máxima:

$$
f=\left\{\frac{\left\langle\sigma_{\max }\right\rangle}{\sigma_{\max }^{0}}\right\}
$$

En este caso $\sigma_{\max }^{0}$ representa la máxima tensión principal admisible y los paréntesis de Macaulay indican que una tensión de compresión pura no inicia el daño.

Criterio de la deformación principal máxima:

$$
f=\left\{\frac{\left\langle\varepsilon_{\max }\right\rangle}{\varepsilon_{\max }^{0}}\right\}
$$

En este caso $\varepsilon_{\max }^{0}$ representa la máxima deformación principal admisible y los paréntesis de Macaulay indican que una deformación de compresión pura no inicia el daño.

En todos los casos se considera que el daño se inicia cuando $f$ alcanza el valor 1 .

Finalmente, es importante aclarar que en el caso del método XFEM una fisura adicional se introduce o una fisura existente se propaga si el criterio de fractura, $f$, alcanza el valor 1 dentro de una determinada tolerancia, ABAQUS (2013a):

$$
1 \leq f \leq 1+f_{\text {tol }}
$$

El valor considerado por defecto para $f_{t o l}$ es 0.05 .

Debe mencionarse que la implementación del modelo en ABAQUS (2013a) con integración implícita presenta ablandamiento y degradación de la rigidez, lo cual origina severos problemas de convergencia. Por este motivo se utiliza una regulación viscosa de las ecuaciones constitutivas que definen el comportamiento cohesivo, ya que el mismo permite que la matriz de rigidez tangente sea positiva para incrementos lo suficientemente pequeños. El valor del parámetro viscoso debe ser tal que la energía disipada por las fuerzas viscosas (ALLVD) sea menor al $10 \%$ de la energía interna. 
CAPítulo 3. Algoritmo desarrollado y modelo numérico aplicado para el estudio del proceso de fisuración en estructuras de hormigón

\section{Aplicación del método XFEM}

En la modelación del problema propuesto se utilizó un criterio de inicio de daño basado en tensiones principales máximas y una ley de ablandamiento lineal, manteniendo lo adoptado en el modelo de plasticidad con daño. Como se explicó anteriormente, para mejorar las condiciones de convergencia se utilizó un coeficiente de estabilización cohesivo (amortiguamiento) igual a 0.01. Se verificó que el mismo no influyera en los resultados obtenidos, ya que la energía de disipación viscosa (ALLVD) es menor al $10 \%$ de la energía interna (ALLIE).

La aplicación del método XFEM consiste en el enriquecimiento de los nodos de la malla de elementos finitos a partir de la definición de una nueva función de forma para los mismos, como se indicó en el Capítulo 2. La geometría del problema analizado está constituida por una única parte, por lo que se aplica un único enriquecimiento en toda la geometría.

En la zona inferior del bloque se generan tensiones altas debido a la restricción provocada por el empotramiento, por lo que se supera el criterio de inicio de daño, lo cual da lugar a la aparición de dos fisuras en los extremos inferiores, como se observa en la Figura 3.19. XFEM solo permite la aparición de una fisura por enriquecimiento, a menos que sean varias fisuras simultáneas, ABAQUS (2013a). Esto significa que en el problema que se analizó no podrán evidenciarse otras fisuras, además de las generadas en los extremos del empotramiento, aunque en otras zonas se alcance el criterio de inicio de daño, ya que ABAQUS (2013a) solo permite definir un enriquecimiento en este problema.

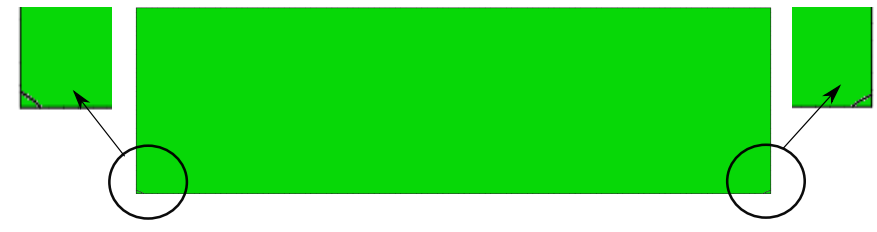

Figura 3.19: Patrón de fisuración obtenido aplicando el método XFEM para el bloque estudiado en el análisis comparativo de modelos numéricos para el estudio del proceso de fisuración

\section{Modelo adoptado}

En la presente tesis se propone analizar problemas de fisuración en estructuras de hormigón sin presencia previa de defectos, por ello el problema que se propuso estudiar para definir el modelo a utilizar fue el de un bloque de hormigón intacto sometido a una contracción térmica.

A partir de los resultados obtenidos puede concluirse que el método XFEM no es adecuado, ya que solo permite que en cada enriquecimiento 
se genere una fisura, o más de una siempre y cuando las mismas ocurran de manera simultánea, ABAQUS (2013a). En el caso analizado solo puede definirse un enriquecimiento, ya que no se dispone de un criterio para determinar una subdivisión que permita mantener la mayor generalidad posible en el problema. Por este motivo al generarse las fisuras en la proximidad de los apoyos debido a un efecto de borde, queda vedada la posibilidad de aparición de una fisura adicional no simultánea con las mismas.

En cuanto a la utilización del Modelo de Fisuración Cohesiva, mediante el empleo de elementos cohesivos, puede decirse que la implementación del mismo en una estructura inicialmente intacta es considerablemente trabajosa, ya que implica la incorporación de elementos cohesivos en todas las interfases de los elementos convencionales. En el problema analizado únicamente se incorporaron en la dirección vertical por las razones expuestas anteriormente, lo cual obliga a la utilización de una malla de elementos finitos relativamente fina con una gran cantidad de elementos, 15075 elementos frente a 4800 elementos en las mallas de los modelos XFEM y de plasticidad con daño, dando lugar a un considerable aumento del tiempo de cálculo. Además los resultados obtenidos con este modelo para el caso analizado, dependen de la cantidad de interfases con elementos cohesivos utilizadas, ya que la estructura se encuentra intacta, es decir, sin fisuras, entallas o defectos previos.

Finalmente si bien el modelo de plasticidad con daño es un modelo continuo que no permite definir el camino de la fisura, sino zonas dañadas, su implementación puede llevarse a cabo con mayor generalidad, al ser aplicado directamente a las propiedades del material en toda la masa de la estructura, sin la necesidad de definir un enriquecimiento en la misma, como en el caso de XFEM o adoptar un criterio para definir la cantidad de interfases con elementos cohesivos a utilizar. Además las zonas dañadas permiten inferir los lugares en donde aparecerán fisuras, como se observa en la Figura 3.18. Por este motivo se decidió adoptar el modelo de plasticidad con daño para estudiar el fenómeno de fisuración por contracción en estructuras de hormigón.

\subsection{Aplicaciones del algoritmo desarrollado}

En las secciones siguientes se aplica el algoritmo desarrollado a distintos problemas con el solo objetivo de mostrar su funcionamiento. Estos problemas son los siguientes: ensayo de tracción directa, contracción térmica uniforme en un elemento de hormigón y gradientes de temperatura sobre elementos de hormigón con distinta relación de aspecto. 
CAPítulo 3. Algoritmo desarrollado y modelo numérico aplicado para el estudio del proceso de fisuración en estructuras de hormigón

\subsubsection{Ensayo de tracción directa}

Se simuló un ensayo de tracción directa sobre una probeta de hormigón simple de 10 x 20 x $50 \mathrm{~cm}$ a la que se le aplicó un desplazamiento de $2 \mathrm{~mm}$ en sus extremos. Las propiedades mecánicas consideradas están definidas en la Tabla 3.1.

La Figura 3.20 muestra el mallado utilizado, constituido por 2000 elementos cuadrados de $5 \mathrm{~mm}$ de lado, de 4 nodos en tensión plana con integración completa (CPS4) y la condición de carga. Para la simulación del ensayo se utilizaron propiedades mecánicas uniformes y distribuidas aleatoriamente adoptando como modelo constitutivo para el hormigón el modelo de plasticidad con daño. En este último caso fueron simuladas 15 asignaciones aleatorias de propiedades mecánicas.

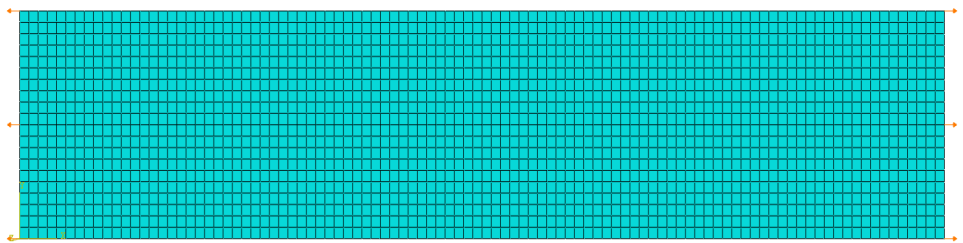

Figura 3.20: Mallado y condición de carga de la probeta de 10 x 20 x $50 \mathrm{~cm}$ sometida al ensayo de tracción directa

Para el caso de la probeta con propiedades mecánicas uniformes, la carga de rotura fue de $57.90 \mathrm{KN}$, correspondiente a una resistencia de 2.89 MPa. En la Tabla 3.5 se presenta la carga de rotura y la tensión correspondiente para cada uno de los casos analizados con distribución aleatoria de las propiedades del material. La tensión se determina como el cociente entre la carga de rotura y la sección transversal de la probeta.

A partir de los resultados que se muestran en la Tabla 3.5 puede observarse que la desviación obtenida en la resistencia a tracción como resultado de la simulación numérica del ensayo de tracción directa es muy baja, correspondiente al $2.93 \%$. Este valor se encuentra dentro de los límites establecidos por los reglamentos para considerar válido un ensayo de laboratorio, ACI (2011, 2014). Por este motivo puede verificarse que los parámetros adoptados para la curva de distribución de Weibull utilizada al asignar aleatoriamente las propiedades mecánicas del hormigón son correctos.

En las Figuras 3.21 y 3.22 se presentan las curvas carga-desplazamiento y tensión-apertura para la probeta con propiedades mecánicas homogéneas y con propiedades mecánicas aleatorias. Para determinar la curva tensiónapertura, la tensión máxima se calcula a partir de la carga máxima, y la apertura como la diferencia entre el desplazamiento al final del ensayo y el desplazamiento correspondiente a la carga máxima.

En la Figura 3.21 puede observarse que la curva carga-desplazamiento 


\begin{tabular}{|c|c|c|}
\hline Caso & Carga de rotura [KN] & Resistencia a la tracción [MPa] \\
\hline 1 & 50.76 & 2.54 \\
\hline 2 & 49.83 & 2.49 \\
\hline 3 & 48.73 & 2.44 \\
\hline 4 & 48.70 & 2.43 \\
\hline 5 & 48.84 & 2.44 \\
\hline 6 & 50.59 & 2.53 \\
\hline 7 & 51.11 & 2.56 \\
\hline 8 & 52.01 & 2.60 \\
\hline 9 & 50.97 & 2.55 \\
\hline 10 & 49.12 & 2.46 \\
\hline 11 & 48.98 & 2.45 \\
\hline 12 & 51.65 & 2.58 \\
\hline 13 & 49.72 & 2.49 \\
\hline 14 & 52.94 & 2.65 \\
\hline 15 & 52.94 & 2.65 \\
\hline Promedio & 50.46 & 2.52 \\
\hline Desviación & 1.48 & 0.07 \\
\hline
\end{tabular}

Tabla 3.5: Carga de rotura y resistencia a tracción para el ensayo de tracción directa obtenidas aplicando distribución aleatoria de las propiedades mecánicas

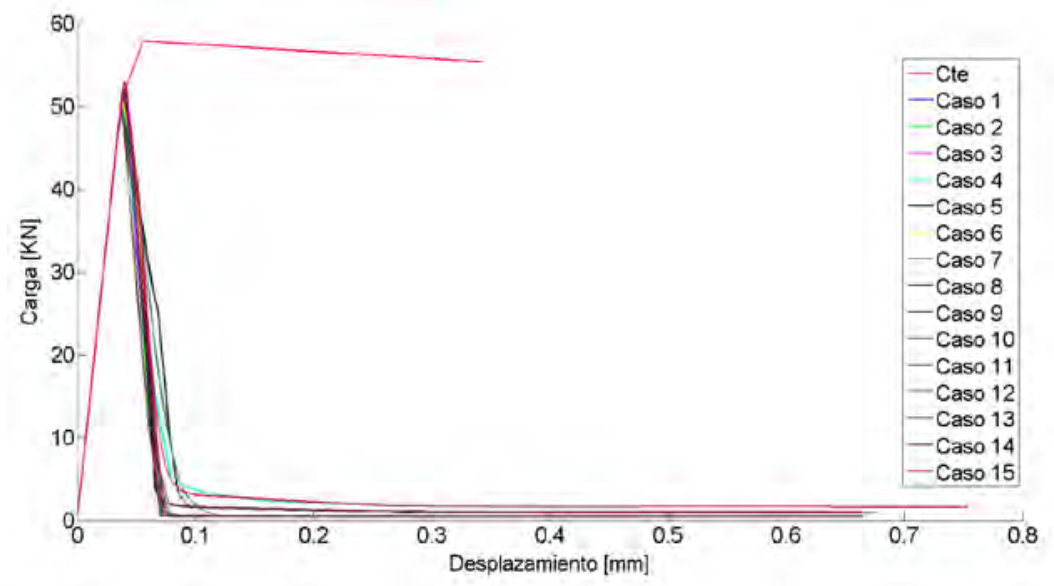

Figura 3.21: Curva carga-desplazamiento del punto de aplicación de la carga en el ensayo de tracción directa para cada uno de los casos analizados

correspondiente a la probeta simulada con propiedades mecánicas uniformes presenta un comportamiento elasto-plástico. Esto no es correcto para un material como el hormigón, el cual presenta un comportamiento cuasi-frágil 
CAPítulo 3. Algoritmo desarrollado y modelo numérico aplicado para el estudio del proceso de fisuración en estructuras de hormigón

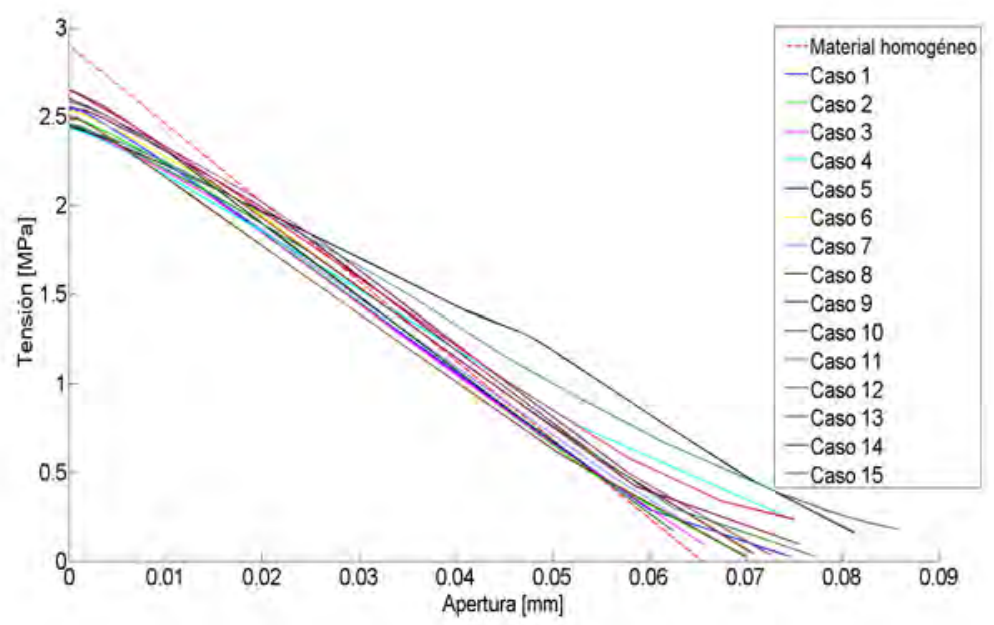

Figura 3.22: Curvas tensión-desplazamiento para cada uno de los casos analizados

frente a cargas de tracción y compresión. Por lo tanto, en el caso de considerar propiedades mecánicas uniformes, en el ensayo de tracción directa, el modelo numérico adoptado no reproduce los resultados que se observan en un ensayo real. Sin embargo, si se observan las curvas carga-desplazamiento obtenidas en los casos en que se realizó asignación aleatoria de propiedades, el comportamiento de las mismas responde al de un material cuasi-frágil. Esto es de suma importancia, ya que si no se asignaran las propiedades mecánicas en forma aleatoria los resultados obtenidos a partir de la simulación numérica del ensayo no serían correctos a pesar de aplicar un modelo de plasticidad con daño.

Por otro lado, en la Figura 3.22 puede observarse que las curvas tensiónapertura para los casos en los que se utilizó distribución aleatoria de las propiedades del material son diferentes entre sí y con respecto a la correspondiente al caso con distribución uniforme de propiedades mecánicas. Esto se debe a que al utilizar el material distribuido aleatoriamente los valores de resistencia a tracción en cada uno de los casos corresponden a distintos puntos de la curva de distribución de probabilidades de Weibull, en cambio para el material uniforme solo se emplea el valor de la resistencia media.

Finalmente en las Figuras 3.23 y 3.24 se presentan las zonas dañadas obtenidas al considerar distribución uniforme de las propiedades mecánicas del material y distribución aleatoria de las mismas, respectivamente.

En la Figura 3.23 puede observarse que se presentan dos zonas dañadas simétricas cercanas a los apoyos debido a la utilización de distribución uniforme de las propiedades mecánicas, lo que no se corresponde con el compor- 

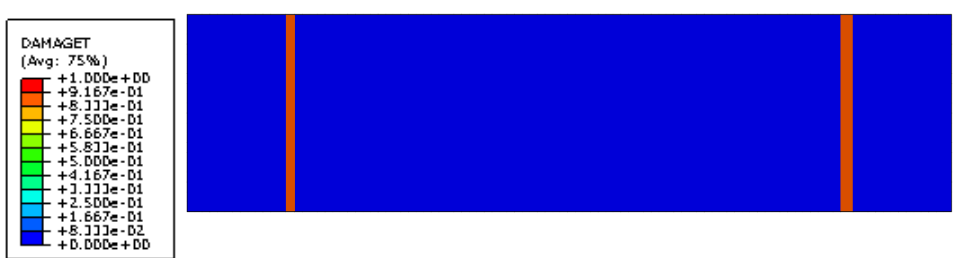

Figura 3.23: Zonas dañadas correspondientes a la probeta de $10 \times 20 \times 50 \mathrm{~cm}$ simulada considerando distribución uniforme de las propiedades mecánicas

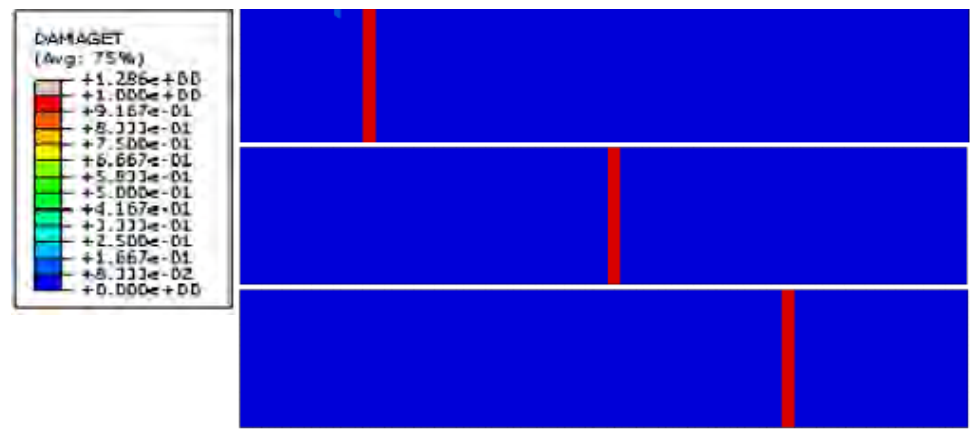

Figura 3.24: Zonas dañadas correspondientes a la probeta de $10 \times 20$ x $50 \mathrm{~cm}$ simulada considerando distribución aleatoria de las propiedades mecánicas

tamiento observado en un ensayo de laboratorio. Por otro lado en la Figura 3.24 se observa que en cada uno de los casos analizados existe una única zona dañada con distinta ubicación, lo cual es más representativo de lo que ocurre en un ensayo de laboratorio.

\subsubsection{Elemento de hormigón sometido a contracción unifor- me}

El bloque de hormigón presentado en la sección 3.3 fue modelado nuevamente considerando que el material posee una distribución tipo Weibull. La geometría, condiciones de contorno, estado de cargas y propiedades del material son las mismas que se utilizaron en la sección anterior. Solamente es necesario indicar que para la distribución de Weibull se adoptó un factor de forma, $m$, igual a 12, de acuerdo con Baẑant y Planas (1997) y el parámetro de escala, $\sigma_{0}$, se calculó a partir de la siguiente expresión, Baẑant y Planas (1997):

$$
\sigma_{0}=\frac{\bar{\sigma}_{0}}{\Gamma\left(1+\frac{1}{m}\right)} \approx 0,6366^{-1 / m} \bar{\sigma}_{0}
$$

donde $\bar{\sigma}_{0}$ es la resistencia media a tracción o compresión, $\Gamma\left(1+\frac{1}{m}\right)$ es la función gama y $m$ es el parámetro de escala referido anteriormente.

Para una resistencia a compresión de $38 \mathrm{MPa}, \sigma_{0}$ adopta un valor de 
CAPítulo 3. Algoritmo desarrollado y modelo numérico aplicado para el estudio del proceso de fisuración en estructuras de hormigón

39.46 MPa.

Con el objetivo de analizar la independencia de los resultados respecto del tamaño de elemento utilizado al aplicar el algoritmo desarrollado, se adoptaron dos mallados con elementos CPS4, cuadriláteros de cuatro nodos en tensión plana, en un caso con tamaño de elemento de $50 \mathrm{~mm}$ de lado (4800 elementos en total) y en el otro de $100 \mathrm{~mm}$ de lado (1200 elementos en total). Cada uno de los casos analizados se resolvió para diez distribuciones aleatorias de propiedades mecánicas para dar un enfoque estadístico al análisis. Por otro lado, se utilizó una malla triangular con elementos CPS3, triangulares aproximadamente equiláteros de tres nodos en tensión plana, de 50 $\mathrm{mm}$ de lado (9858 elementos en total) y de $100 \mathrm{~mm}$ de lado (2510 elementos en total). En la Figura 3.25 se observan las mallas utilizadas y en la Figura 3.26 las asignaciones aleatorias de resistencia para el mallado cuadrado con elementos de $50 \mathrm{~mm}$ de lado en tres de los diez casos analizados.
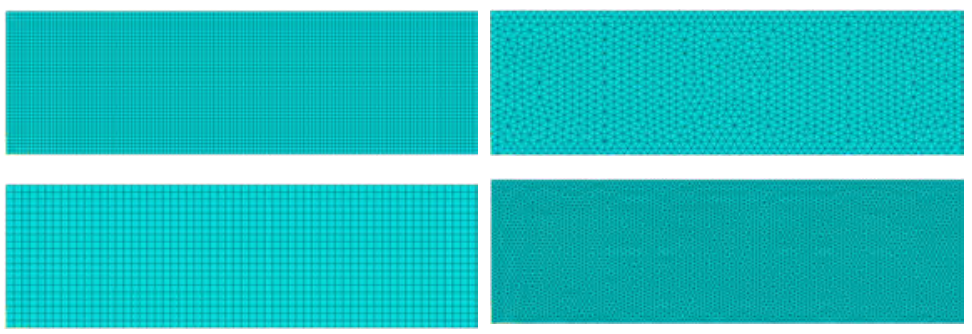

Figura 3.25: Mallados utilizados en la modelación del bloque analizado con el objetivo de estudiar la independencia de malla aplicando el algoritmo desarrollado

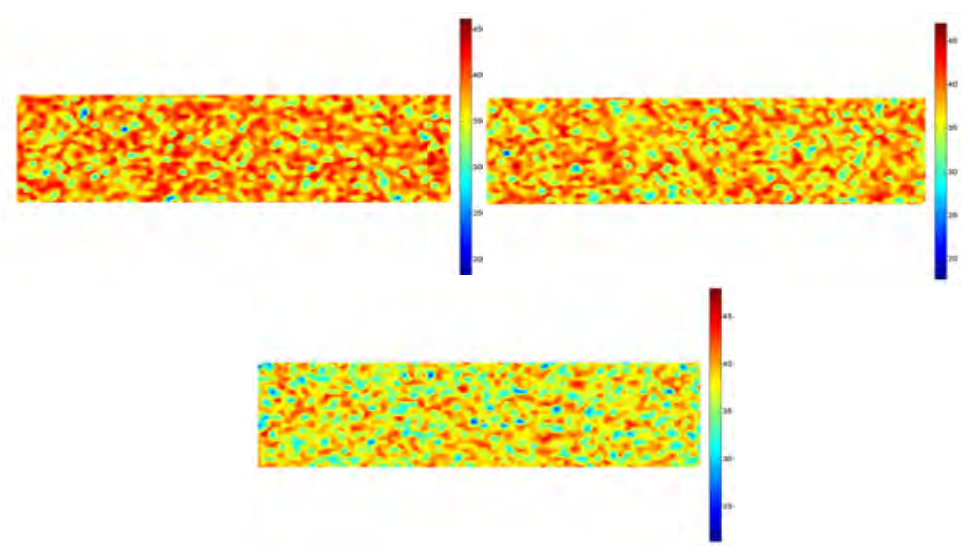

Figura 3.26: Distribuciones aleatorias de propiedades mecánicas para el bloque analizado

Para determinar la edad de fisuración se obtuvo la deformación plástica equivalente en tracción, Lubliner et al. (1989). 


\section{Edad de fisuración}

\section{Resultados para el mallado rectangular}

En la sección 3.3 se realizó un análisis de sensibilidad de malla para el modelo de plasticidad con daño utilizado. En la Tabla 3.3 se observa que el tiempo de aparición de la fisura para el mallado de elementos cuadrados de $50 \mathrm{~mm}$ de lado es de 32.16 horas. En el caso de una malla de elementos cuadrados de $100 \mathrm{~mm}$, la edad de aparición de la fisura es 42.24 horas, lo que significa una diferencia del $23.86 \%$.

En las Tablas 3.6 y 3.7 se muestran las edades de fisuración para las mismas mallas pero considerando 10 distribuciones aleatorias diferentes de propiedades mecánicas. A su vez también se indica el valor medio.

\begin{tabular}{|c|c|c|c|c|c|c|}
\hline \multirow{2}{*}{ Caso } & \multicolumn{7}{|c|}{ Edad de fisuración [horas] } \\
\cline { 2 - 7 } & Fisura 1 & Fisura 2 & Fisura 3 & Fisura 4 & Fisura 5 & Fisura 6 \\
\hline 1 & 28.8 & 34.8 & 61.68 & 103.68 & - & - \\
\hline 2 & 25.2 & 27.12 & 77.52 & 79.44 & - & - \\
\hline 3 & 30 & 52.32 & 58.32 & 75.12 & 83.04 & - \\
\hline 4 & 27.6 & 32.88 & 33.84 & 88.8 & - & - \\
\hline 5 & 30.24 & 35.52 & 49.92 & 54.48 & 92.4 & - \\
\hline 6 & 24.24 & 35.28 & 54.24 & 63.36 & 91.44 & - \\
\hline 7 & 28.08 & 34.8 & 49.92 & 61.92 & 92.88 & - \\
\hline 8 & 28.56 & 29.28 & 42.48 & 61.44 & 89.76 & 101.76 \\
\hline 9 & 27.84 & 42 & 46.32 & 90.48 & - & - \\
\hline 10 & 30.24 & 36.72 & 54.96 & 93.6 & - & - \\
\hline Promedio & 28.08 & 36.07 & 52.92 & 77.23 & 89.90 & 101.76 \\
\hline
\end{tabular}

Tabla 3.6: Edad de fisuración para un bloque con distribución de propiedades mecánicas aleatoria para una malla cuadrada con elementos de $50 \mathrm{~mm}$ de lado

A partir de las Tablas 3.6 y 3.7, puede observarse que la edad de fisuración obtenida utilizando una malla con elementos cuadrados de $50 \mathrm{~mm}$ presenta una diferencia en comparación con la malla de elementos cuadrados de 100 $\mathrm{mm}$ de $3.62 \%$ para la primera fisura, $15.90 \%$ para la segunda fisura y $14.10 \%$ para la tercera fisura. Estos porcentajes son menores para la primera fisura que los obtenidos utilizando propiedades mecánicas uniformes.

Es importante mencionar que al aplicar un modelo de plasticidad con daño a estructuras sin armadura o con poca armadura, la especificación de una relación tensión-deformación luego de la falla introduce sensibilidad de malla en los resultados, ya que dichos resultados no convergen a una solución única a medida que la malla se refina debido a que este refinamiento conduce a bandas de fisuración más estrechas. Esto ocurre si la fisuración tiene lugar solo en regiones localizadas de la estructura y el refinamiento de la malla no 
CAPítulo 3. Algoritmo desarrollado y modelo numérico aplicado para el estudio del proceso de fisuración en estructuras de hormigón

\begin{tabular}{|c|c|c|c|c|c|}
\hline \multirow{2}{*}{ Caso } & \multicolumn{5}{|c|}{ Edad de fisuración [horas] } \\
\cline { 2 - 6 } & Fisura 1 & Fisura 2 & Fisura 3 & Fisura 4 & Fisura 5 \\
\hline 1 & 30.48 & 31.44 & 85.44 & 112.8 & - \\
\hline 2 & 29.76 & 47.04 & 48 & 66.72 & 75.6 \\
\hline 3 & 28.08 & 42.48 & 53.52 & 105.6 & - \\
\hline 4 & 32.16 & 53.04 & 82.56 & 104.4 & 119.28 \\
\hline 5 & 28.08 & 40.32 & 54.96 & 85.2 & 91.2 \\
\hline 6 & 29.28 & 30 & 78.72 & 116.64 & - \\
\hline 7 & 28.08 & 47.76 & 50.64 & - & - \\
\hline 8 & 31.2 & 48.24 & 56.16 & 89.28 & 110.88 \\
\hline 9 & 28.08 & 32.40 & 43.68 & 69.36 & 104.4 \\
\hline 10 & 26.16 & 45.36 & 50.16 & 74.16 & - \\
\hline Promedio & 29.14 & 41.81 & 60.38 & 91.57 & 100.27 \\
\hline
\end{tabular}

Tabla 3.7: Edad de fisuración para un bloque con distribución de propiedades mecánicas aleatoria para una malla cuadrada con elementos de $100 \mathrm{~mm}$ de lado

resulta en la formación de fisuras adicionales. Si la fisuración se distribuye uniformemente, debido a la presencia de armaduras, la sensibilidad de malla es menor, ABAQUS (2013b). Para resolver este problema ABAQUS (2013b) propone caracterizar el comportamiento en tracción del hormigón mediante una respuesta tensión-desplazamiento en el caso de estructuras de hormigón sin armar.

Sin embargo en el bloque analizado con malla de elementos cuadrados a pesar de haber utilizado una curva tensión-desplazamiento se observa dependencia del tamaño de elementos de la malla con los resultados, pero al considerar propiedades mecánicas distribuidas aleatoriamente esa dependencia se reduce. La razón de este comportamiento puede estar relacionado con que la aleatoriedad permite una localización más uniforme de las fisuras en la estructura.

\section{Resultados para el mallado triangular}

En la Tabla 3.4 se observa que el tiempo de aparición de la fisura para el mallado de elementos triangulares de $50 \mathrm{~mm}$ de lado con distribución uniforme de la propiedades mecánicas es 35.22 horas. En el caso de una malla de elementos triangulares de $100 \mathrm{~mm}$, la edad de aparición de la fisura es 38.89 horas. La diferencia entre los resultados obtenidos utilizando la malla fina y la gruesa es de $7.4 \%$.

En las Tablas 3.8 y 3.9 se muestran las edades de fisuración para las mismas mallas pero en este caso considerando 10 asignaciones aleatorias de propiedades mecánicas. A su vez también se indica el valor medio. 
3.4. Aplicaciones del algoritmo desarrollado

\begin{tabular}{|c|c|c|c|c|}
\hline \multirow{2}{*}{ Caso } & \multicolumn{4}{|c|}{ Edad de fisuración [horas] } \\
\cline { 2 - 5 } & Fisura 1 & Fisura 2 & Fisura 3 & Fisura 4 \\
\hline 1 & 30.24 & 39.84 & 53.52 & - \\
\hline 2 & 34.56 & 38.64 & 56.16 & 68.88 \\
\hline 3 & 30.24 & 53.04 & 102.24 & - \\
\hline 4 & 37.2 & 39.36 & 41.76 & - \\
\hline 5 & 32.16 & 38.16 & 38.16 & - \\
\hline 6 & 32.88 & 36.72 & 38.64 & 65.52 \\
\hline 7 & 36.96 & 40.8 & 46.56 & - \\
\hline 8 & 30.24 & 36 & 51.36 & 54.72 \\
\hline 9 & 36 & 36 & 105.6 & - \\
\hline 10 & 32.16 & 34.08 & 55.92 & 88.32 \\
\hline Promedio & 33.26 & 39.26 & 59 & 69.36 \\
\hline
\end{tabular}

Tabla 3.8: Edad de fisuración para un bloque con distribución de propiedades mecánicas aleatoria para una malla triangular con tamaño de elementos de $50 \mathrm{~mm}$ de lado

\begin{tabular}{|c|c|c|c|c|}
\hline \multirow{2}{*}{ Caso } & \multicolumn{5}{|c|}{ Edad de fisuración [horas] } \\
\cline { 2 - 5 } & Fisura 1 & Fisura 2 & Fisura 3 & Fisura 4 \\
\hline 1 & 36 & 44.16 & - & - \\
\hline 2 & 38.16 & 40.32 & 44.16 & - \\
\hline 3 & 37.2 & 56.64 & 99.12 & - \\
\hline 4 & 30.24 & 40.32 & 62.88 & - \\
\hline 5 & 40.08 & 42.24 & - & - \\
\hline 6 & 38.64 & 44.16 & - & - \\
\hline 7 & 38.16 & 40.32 & - & - \\
\hline 8 & 36 & 36 & - & - \\
\hline 9 & 32.16 & 36 & 43.44 & 48.96 \\
\hline 10 & 40.32 & 40.32 & - & - \\
\hline Promedio & 36.7 & 42.05 & 62.4 & 48.96 \\
\hline
\end{tabular}

Tabla 3.9: Edad de fisuración para un bloque con distribución de propiedades mecánicas aleatoria para una malla triangular con tamaño de elementos de $100 \mathrm{~mm}$ de lado

A partir de las Tablas 3.8 y 3.9, se observa que la edad de fisuración obtenida utilizando una malla con elementos de $50 \mathrm{~mm}$ presenta una diferencia en comparación con la malla de elementos de $100 \mathrm{~mm}$ de $9.4 \%$ para la primera fisura y $6.6 \%$ para la segunda fisura. Para el caso de la primera fisura, el porcentaje obtenido utilizando material con distribución aleatoria es ligeramente superior al obtenido considerando distribución uniforme. A diferencia de lo que sucedía al utilizar elementos cuadrados, al emplear 
CaPítulo 3. Algoritmo desarrollado y modelo numérico aplicado para el estudio del proceso de fisuración en estructuras de hormigón

elementos triangulares no se presenta una pronunciada diferencia, en cuanto a dependencia de malla, al aplicar distribución uniforme o distribución aleatoria de las propiedades mecánicas del material.

\section{Zonas dañadas}

En las Figuras 3.27 y 3.28 se observan las zonas dañadas para el bloque con distribución uniforme de las propiedades mecánicas del material, tanto para el mallado cuadrado como para el triangular.

Por otro lado en las Figuras 3.29 y 3.30 se presentan las zonas dañadas para el bloque con distribución aleatoria de las propiedades del material para los 10 casos analizados, correspondientes al mallado cuadrado y triangular, respectivamente. En ambos casos se consideró el mallado más fino.

Como se esperaba, las zonas dañadas obtenidas cambian para las diferentes asignaciones aleatorias de propiedades mecánicas, en comparación con la distribución uniforme de propiedades.

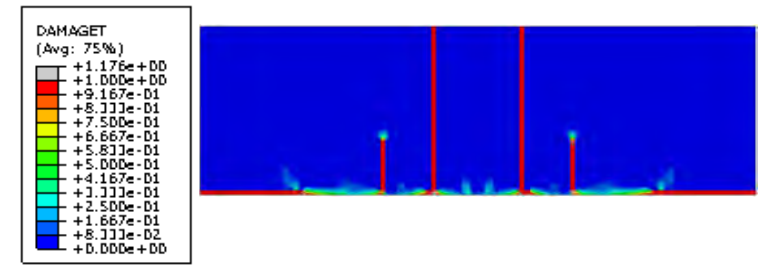

Figura 3.27: Zonas dañadas para el bloque de hormigón sometido a una contracción térmica uniforme con distribución uniforme de las propiedades mecánicas y mallado cuadrado

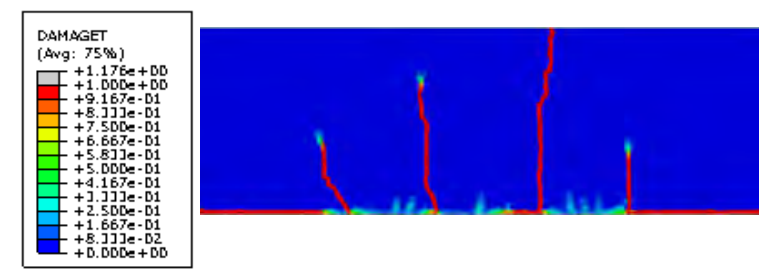

Figura 3.28: Zonas dañadas para el bloque de hormigón sometido a una contracción térmica uniforme con distribución uniforme de las propiedades mecánicas y mallado triangular

\subsubsection{Elementos de hormigón sometidos a gradientes de tem- peratura}

Para analizar numéricamente un problema de contracción térmica en el cual las fisuras se generan como resultado de gradientes de masa y de 


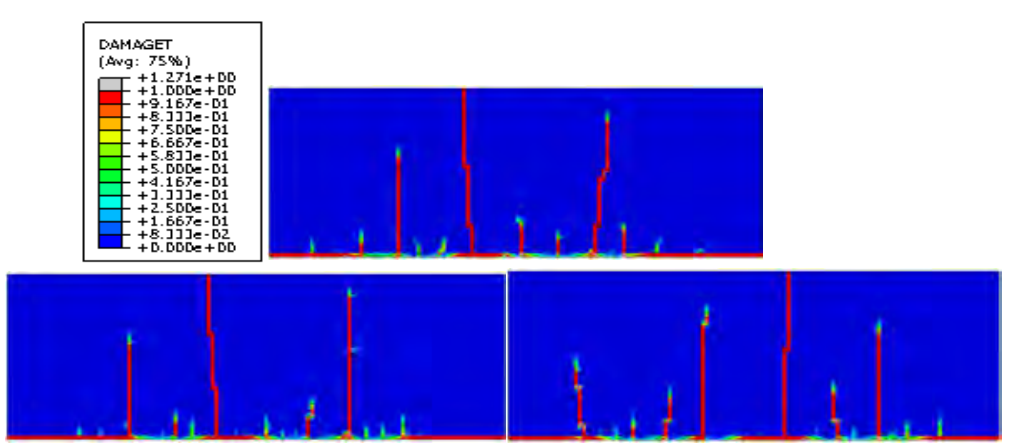

Figura 3.29: Zonas dañadas para el bloque de hormigón sometido a una contracción térmica uniforme con distribución aleatoria de las propiedades mecánicas y mallado cuadrado

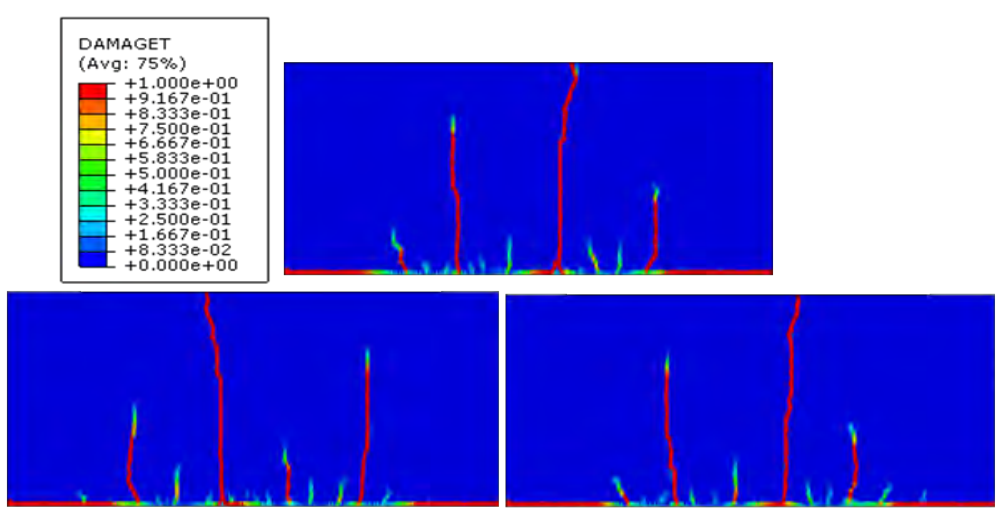

Figura 3.30: Zonas dañadas para el bloque de hormigón sometido a una contracción térmica uniforme con distribución aleatoria de las propiedades mecánicas y mallado triangular

superficie, es necesario realizar dos tipos de análisis. En primer lugar se debe llevar a cabo un análisis térmico en el que interviene, como estado de carga, la curva de elevación adiabática de temperatura en el hormigón. Para ello dicha curva se transforma en una curva de flujo de calor volumétrico a partir de la expresión 3.51:

$$
\frac{d T}{d t}=\frac{d Q}{d t}\left(\frac{1}{\rho * c}\right)
$$

donde $\rho$ es la densidad del hormigón y $c$ el calor específico. En la Figura 3.31 se observa la curva de elevación adiabática utilizada en los problemas analizados, correspondientes a un contenido unitario de cemento de $400 \mathrm{Kg} / \mathrm{m}^{3}$.

Las condiciones de contorno térmicas que se utilizan en este tipo de análisis pueden ser convección, radiación, insolación y evaporación. En este caso únicamente se consideró la convección entre el hormigón y el aire, adoptando un coeficiente de convección igual a $1,6 * 10^{-9} \mathrm{Kcal} / \mathrm{mm}^{2} \mathrm{seg}^{\circ} \mathrm{C}$. La tempe- 
CAPítulo 3. Algoritmo desarrollado y modelo numérico aplicado para el estudio del proceso de fisuración en estructuras de hormigón

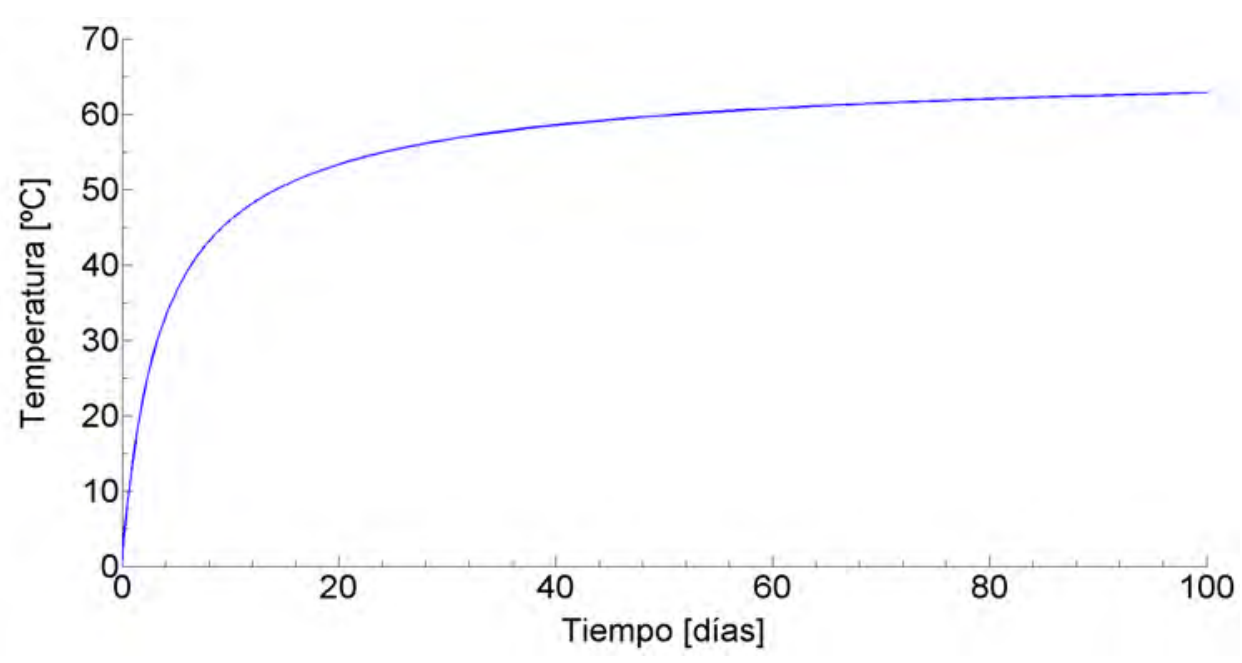

Figura 3.31: Curva de elevación de temperatura adiabática utilizada en los análisis térmicos para el estudio de la fisuración por contracción térmica debido a gradientes de temperatura

ratura ambiente se adoptó constante e igual a $15^{\circ} \mathrm{C}$ y la temperatura de colocación del hormigón igual a $30^{\circ} \mathrm{C}$.

A partir del modelo térmico se obtiene la evolución de temperatura en el tiempo en cada nodo de la estructura, lo que constituyen el estado de carga del modelo mecánico, a partir del cual se determinan las tensiones y el tiempo de aparición de las fisuras.

Para el modelo mecánico se empleó el mismo mallado que para el modelo térmico. En todos los casos la duración de ambos análisis fue de 28 días.

Las propiedades mecánicas utilizadas son las que se detallan en la Tabla 3.1. Los casos estudiados corresponden a dos elementos de hormigón con relación de aspecto igual a $5(50 \times 10 \mathrm{~cm}$ y 250 x $50 \mathrm{~cm})$ y un elemento con relación de aspecto igual a $1(50 \times 50 \mathrm{~cm})$. Estas geometrías fueron seleccionadas con el objetivo de determinar la influencia de la forma del elemento estructural en la disipación de calor y la formación de fisuras de origen térmico.

Para poder estudiar el estado tensional de una estructura bajo la acción de gradientes térmicos es necesario considerar la evolución en el tiempo de las propiedades mecánicas. En 3.52 se indican las expresiones utilizadas para calcular módulo de elasticidad, resistencia a compresión y resistencia a tracción, CEB-FIB (1993):

$$
\begin{gathered}
E(t)=E_{28} *\left(e^{0,28 *\left(1-\left(\frac{28}{t}\right)^{0,5}\right.}\right)^{0,5} \\
f_{c}(t)=f_{c 28} *\left(e^{0,28 *\left(1-\left(\frac{28}{t}\right)^{0,5}\right.}\right)^{0,5} \\
f_{c t}(t)=1,34 *\left(\left(\left(f_{c}(t) / 12\right)^{0,33}\right)^{2}\right)
\end{gathered}
$$


donde $E(t)$ es el módulo de elasticidad variable en el tiempo, $E_{28}$ es el módulo de elasticidad a 28 días, $f_{c}(t)$ es la resistencia a compresión variable en el tiempo, $f_{c 28}$ es la resistencia a compresión a 28 días y $f_{c t}(t)$ es la resistencia a tracción variable en el tiempo, con $t$ expresado en días.

En todos los casos se utilizó en el modelo mecánico un mallado compuesto de elementos cuadrados de cuatro nodos en tensión plana con integración reducida (CPS4) y en el modelo térmico por elementos de cuatro nodos lineales de transferencia de calor (DC2D4). Para la geometría de 50 x $10 \mathrm{~cm}$ se emplearon 2000 elementos, para la de 50 x $250 \mathrm{~cm} 3125$ elementos y para la de 50 x $50 \mathrm{~cm} 2500$ elementos. En el modelo térmico se consideró que el intercambio de calor con el ambiente (convección) se produce a través de las caras superior e inferior y no por los laterales, y en el modelo mecánico se aplicaron empotramientos en las caras laterales.

\section{Resultados del análisis térmico}

En la Figura 3.32 se presenta la evolución de temperatura del elemento de $10 \times 50 \mathrm{~cm}$ (relación de aspecto 5), para las edades de 1 h, 10 hs, 24 hs y 28 días. En esta figura puede observarse que el enfriamiento del elemento ocurre a edad muy temprana, ya que la temperatura inicial de 30 grados se reduce en 10 horas a 20 grados. Esto se relaciona con que la masa de hormigón en el mismo es pequeña y presenta una gran superficie expuesta, por lo que el calor se disipa rápidamente por convección entre la superficie del elemento y el aire.

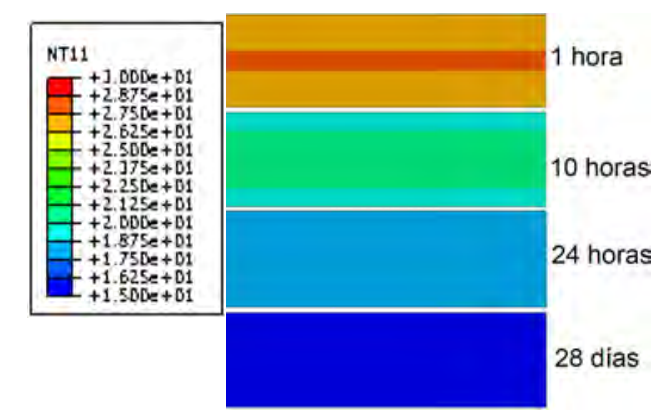

Figura 3.32: Evolución de temperatura en el elemento de 10 x $50 \mathrm{~cm}$ biempotrado, edades $1 \mathrm{~h}, 10 \mathrm{hs}, 24 \mathrm{hs}$ y 28 días

Por otro lado en la En la Figura 3.33 se presenta la evolución de temperatura en el elemento de dimensiones 50 x $250 \mathrm{~cm}$ (relación de aspecto 5) para las edades de 1, 3, 7 y 28 días. En este caso, a diferencia de lo ocurrido con el elemento de $10 \times 50 \mathrm{~cm}$, el enfriamiento de la pieza ocurre lentamente, ya que después de transcurridos 7 días de análisis comienza a estabilizarse térmicamente.

Finalmente en la Figura 3.34 se presenta la evolución de temperatura en 
CAPítulo 3. Algoritmo desarrollado y modelo numérico aplicado para el estudio del proceso de fisuración en estructuras de hormigón

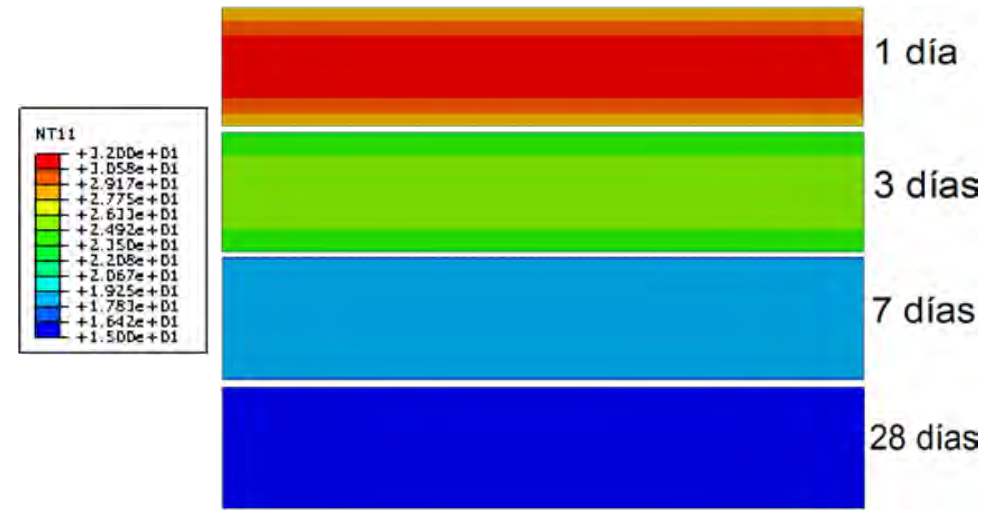

Figura 3.33: Evolución de temperatura en elemento de 50 x $250 \mathrm{~cm}$ biempotrado, edades 1, 3, 7 y 28 días

el elemento de 50 x $50 \mathrm{~cm}$ (relación de aspecto 1) para las edades de 1, 3, 7 y 28 días. En este caso, a diferencia de lo ocurrido con el elemento de 10 x $50 \mathrm{~cm}$ y al igual que en el elemento de 50 x $250 \mathrm{~cm}$, el enfriamiento de la pieza ocurre lentamente, ya que después de transcurridos 7 días de análisis es cuando comienza a estabilizarse térmicamente.

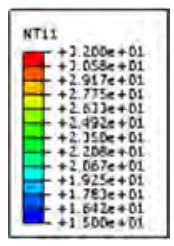

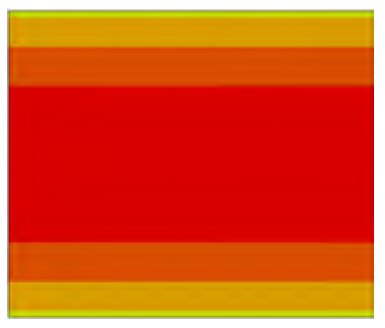

1 día

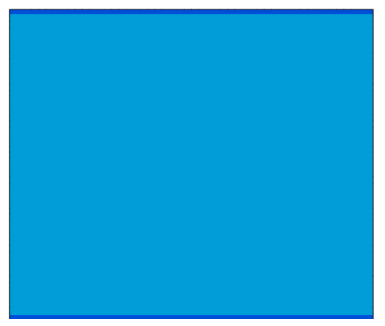

7 días

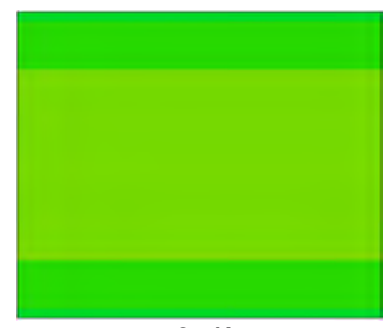

3 días

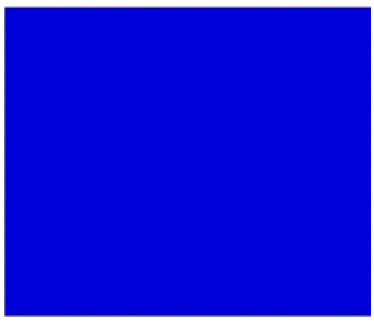

28 días

Figura 3.34: Evolución de temperatura en elemento de $50 \times 50 \mathrm{~cm}$ biempotrado, edades $1,3,7$ y 28 días 


\section{Resultados del análisis mecánico}

Para todas las geometrías se analizaron once casos, uno con propiedades mecánicas uniformes y los restantes con propiedades mecánicas aleatorias utilizando el algoritmo desarrollado, con el objetivo de determinar la edad de fisuración y las zonas dañadas.

Para el elemento de $10 \times 50 \mathrm{~cm}$ en el caso de emplear propiedades mecánicas uniformes no se determina la aparición de fisuras. Pero al utilizar distribución aleatoria de las propiedades del material se observan fisuras en todos los casos, en la Tabla 3.10 se indica el tiempo de aparición.

\begin{tabular}{|c|c|}
\hline Caso & Edad de fisuración [horas] \\
\hline 1 & 541.68 \\
\hline 2 & 145.44 \\
\hline 3 & 203.55 \\
\hline 4 & 277.68 \\
\hline 5 & 324.96 \\
\hline 6 & 311.04 \\
\hline 7 & 372.24 \\
\hline 8 & 163.92 \\
\hline 9 & 322.32 \\
\hline 10 & 142.56 \\
\hline Promedio & 280.54 \\
\hline Desviación & 123.86 \\
\hline
\end{tabular}

Tabla 3.10: Edad de fisuración para un elemento de 10 x $50 \mathrm{~cm}$ biempotrado con distribución de propiedades mecánicas aleatoria sometida a gradientes de temperatura

La desviación estándar presentada en la Tabla 3.10 es alta e igual a 123.86 horas, esto indica una gran dispersión de los resultados obtenidos.

En las Figuras 3.35 y 3.36 se observa la variable de daño al final del análisis para el elemento de $10 \times 50 \mathrm{~cm}$ con propiedades mecánicas uniformes y con propiedades mecánicas aleatorias, respectivamente. En el primer caso esta variable no alcanza el valor 1 , lo cual indica que el elemento no se encuentra fisurado, en cambio para los casos simulados con propiedades mecánicas aleatorias la variable de daño alcanza siempre el valor 1, es decir, el elemento está fisurado.

Las zonas dañadas se presentan en diferentes ubicaciones para cada uno de los casos simulados como resultado de la utilización de las propiedades mecánicas distribuidas aleatoriamente.

Las fisuras del elemento de $10 \times 50 \mathrm{~cm}$ son de superficie, es decir, se producen desde la superficie del elemento hacia el interior, como se observa en la Figura 3.37 ya que en esta dirección ocurre una rápida disipación de 
CaPítulo 3. Algoritmo desarrollado y modelo numérico aplicado para el estudio del proceso de fisuración en estructuras de hormigón

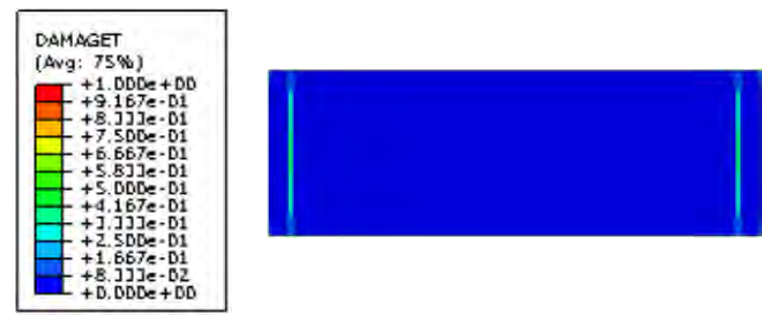

Figura 3.35: Variable de daño para el elemento de 10 x $50 \mathrm{~cm}$ biempotrado simulado con propiedades mecánicas uniformes

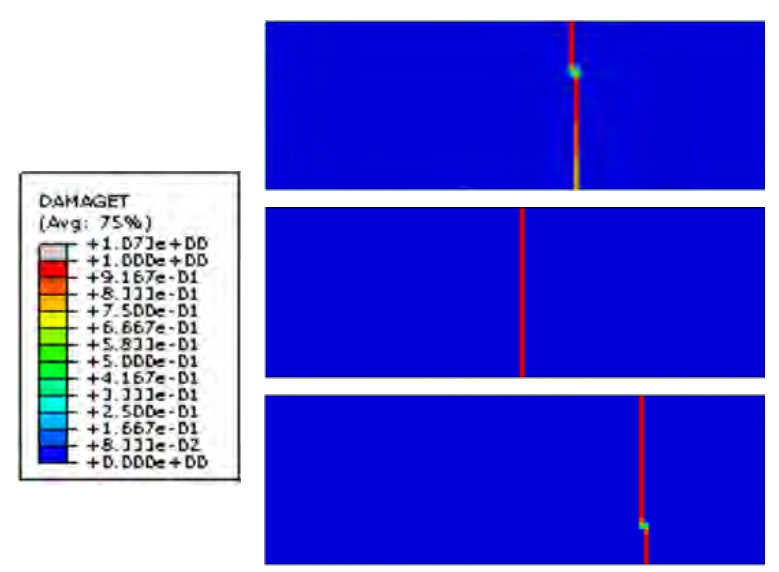

Figura 3.36: Variable de daño para el elemento de $10 \times 50 \mathrm{~cm}$ biempotrado simulado con propiedades mecánicas aleatorias

calor.

Si manteniendo la relación de aspecto se aumenta una de las dimensiones, en lugar de una geometría de 10 x $50 \mathrm{~cm}$ se modela una geometría de $50 \mathrm{x}$ $250 \mathrm{~cm}$, para el caso con propiedades mecánicas uniformes aparecen fisuras a la edad de 3.95 días, a diferencia de lo que ocurría para la geometría de $10 \times 50 \mathrm{~cm}$. Al aplicar propiedades mecánicas distribuidas aleatoriamente se presentan fisuras en todos los casos, como se indica en la Tabla 3.11, al igual que para la geometría de $10 \times 50 \mathrm{~cm}$.

A diferencia de lo ocurrido con el elemento de $10 \times 50 \mathrm{~cm}$, las edades de fisuración son más tardías para el elemento de 50 x $250 \mathrm{~cm}$, 3.37 días en promedio, y la dispersión de resultados más baja, ya que la desviación estándar representa el $44 \%$ del valor promedio en el caso de la geometría de $10 \times 50 \mathrm{~cm}$ pero el $6 \%$ en el caso de la geometría de 50 x $250 \mathrm{~cm}$. Para la misma relación de aspecto, al aumentar las dimensiones de la geometría el calor tarda más en disiparse, por lo que las tensiones máximas tardan más en alcanzarse.

En la Figura 3.38 se presentan las zonas dañadas para la geometría de 


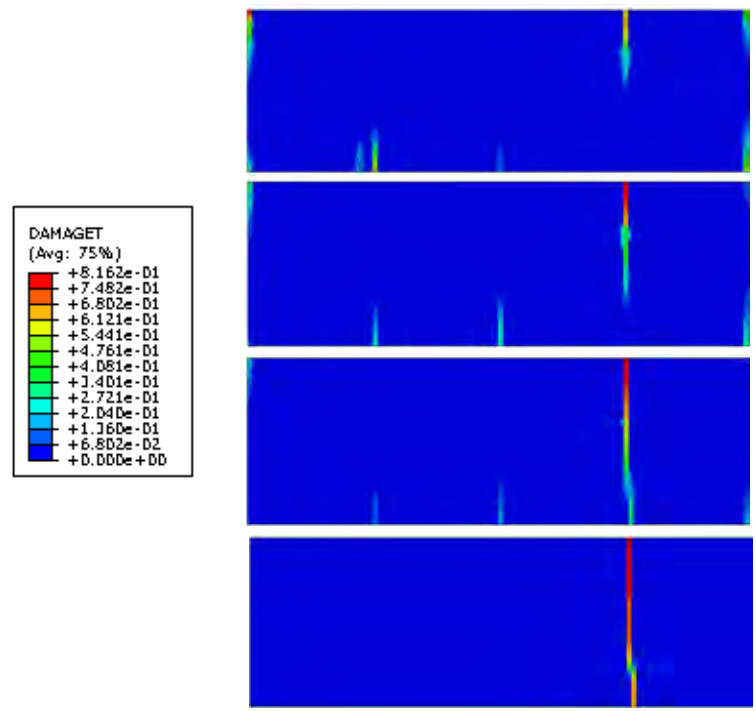

Figura 3.37: Evolución del crecimiento de la zona dañada

\begin{tabular}{|c|c|}
\hline Caso & Edad de fisuración [días] \\
\hline 1 & 3.60 \\
\hline 2 & 3.25 \\
\hline 3 & 3.59 \\
\hline 4 & 3.34 \\
\hline 5 & 2.93 \\
\hline 6 & 3.51 \\
\hline 7 & 3.43 \\
\hline 8 & 3.23 \\
\hline 9 & 3.43 \\
\hline 10 & 3.34 \\
\hline Desviación & 0.19 \\
\hline Promedio & 3.37 \\
\hline
\end{tabular}

Tabla 3.11: Edad de fisuración para el elemento biempotrado de 50 x $250 \mathrm{~cm}$ con distribución de propiedades mecánicas aleatoria sometido a gradientes de temperatura

50 x $250 \mathrm{~cm}$ con propiedades mecánicas uniformes. Se observan cercanas a los bordes, donde existe una fuerte restricción por efecto de la condición de contorno, y en forma simétrica, lo cual se relaciona con la distribución uniforme de la propiedades mecánicas.

Las zonas dañadas obtenidas al aplicar distribución aleatoria de las propiedades mecánicas se observan en la Figura 3.39. Éstas se encuentran en distintos lugares de la viga, y al igual que en la geometría de 10 x $50 \mathrm{~cm}$, 
CAPítulo 3. Algoritmo desarrollado y modelo numérico aplicado para el estudio del proceso de fisuración en estructuras de hormigón

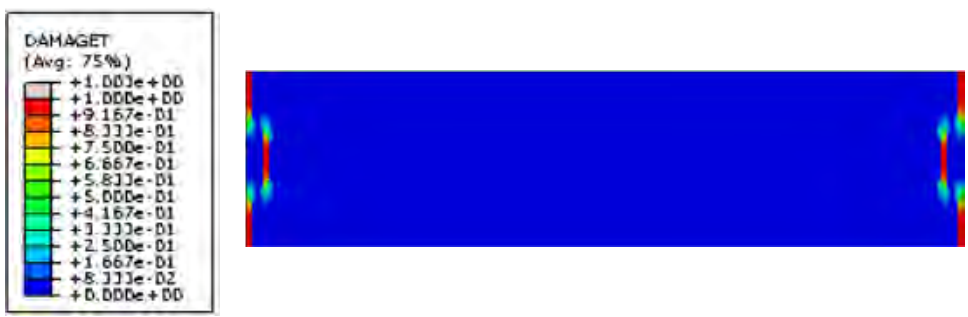

Figura 3.38: Variable de daño para el elemento de $50 \times 250 \mathrm{~cm}$ biempotrado simulado con propiedades mecánicas uniformes

las fisuras crecen desde los bordes extremos al centro de la viga. Esto se debe a que las fisuras se generan por gradientes de superficie, como se indicó anteriormente.

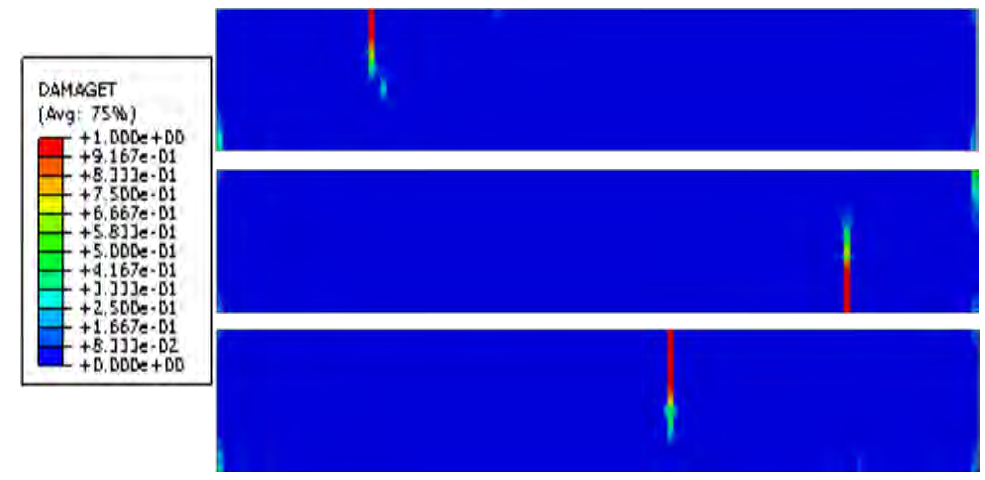

Figura 3.39: Variable de daño para el elemento de $50 \times 250 \mathrm{~cm}$ biempotrado simulado con propiedades mecánicas aleatorias

Es interesante mencionar que si bien la edad de fisuración calculada empleando distribución uniforme de propiedades mecánicas (3.95 días) se aproxima al valor promedio obtenido aplicando distribución aleatoria de las propiedades (3.37 días), los resultados del análisis con propiedades uniformes no son correctos, ya que las zonas dañadas resultantes de la aplicación de propiedades mecánicas uniformes se encuentran simétricamente localizadas cercanas a los bordes, donde existe una fuerte restricción debida a la condición de contorno. En cambio al emplear propiedades distribuidas aleatoriamente las zonas dañadas no presentan ese comportamiento, si no que se distribuyen a lo largo de la superficie del elemento.

Finalmente si se realiza el análisis mecánico en la geometría con relación de aspecto igual a 1, de dimensiones $50 \times 50 \mathrm{~cm}$, aplicando propiedades mecánicas uniformes no se presentan fisuras, como se observa en la Figura 3.40 , ya que la variable de daño no alcanza el valor 1. Esto mismo ocurre en la geometría de $10 \times 50 \mathrm{~cm}$.

A diferencia de lo ocurrido con las geometrías con relación de aspecto 


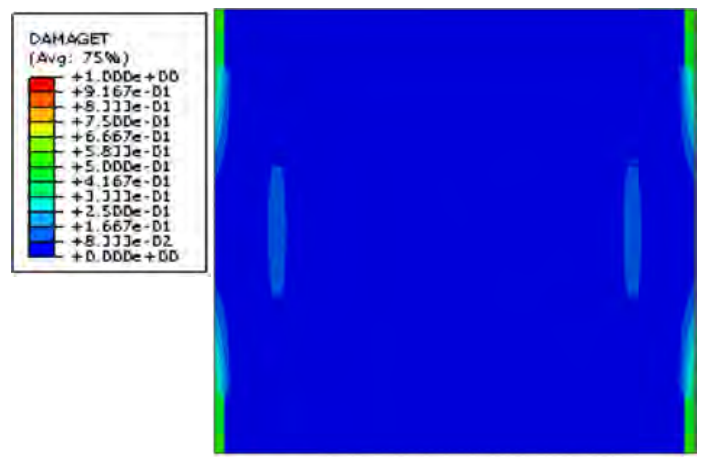

Figura 3.40: Variable de daño para la geoemtría de 50 x $50 \mathrm{~cm}$ biempotrada simulada con propiedades mecánicas uniformes

igual a 5, al observar los resultados obtenidos aplicando distribución aleatoria de las propiedades mecánicas, solo en algunos de los casos analizados $(2,3$, 5 , y 9) aparecen fisuras, como se indica en la Tabla 3.12. En la Figura 3.41 se presentan las zonas dañadas para los casos $2,3,5$ y 9 .

\begin{tabular}{|c|c|}
\hline Caso & Edad de fisuración [días] \\
\hline 1 & No fisuró \\
\hline 2 & 7.42 \\
\hline 3 & 10.58 \\
\hline 4 & No fisuró \\
\hline 5 & 12 \\
\hline 6 & No fisuró \\
\hline 7 & No fisuró \\
\hline 8 & No fisuró \\
\hline 9 & 8.41 \\
\hline 10 & No fisuró \\
\hline
\end{tabular}

Tabla 3.12: Edad de fisuración para la geometría de 50 x $50 \mathrm{~cm}$ biempotrada con distribución de propiedades mecánicas aleatorias sometido a gradientes de temperatura

Además resulta interesante observar que en los casos en que aparecen fisuras (casos 2, 3, 5 y 9) los tiempos de aparición son mayores que los obtenidos para la geometría de $10 \times 50 \mathrm{~cm}$ y del mismo orden de magnitud a los correspondientes a la geometría de $50 \times 250 \mathrm{~cm}$. Esto se relaciona con que tanto la geometría de $50 \times 50 \mathrm{~cm}$ como la de $50 \times 250 \mathrm{~cm}$ son más grandes que la de $10 \times 50 \mathrm{~cm}$, por lo que se requiere más tiempo para que se produzca la disipación de calor a través de la superficie. Como la temperatura más alta se encuentra en el centro de la geometría con relación de aspecto igual a 1 , en la mayoría de los casos las fisuras tienden a localizarse en esta zona y 
CAPítulo 3. Algoritmo desarrollado y modelo numérico aplicado para el estudio del proceso de fisuración en estructuras de hormigón

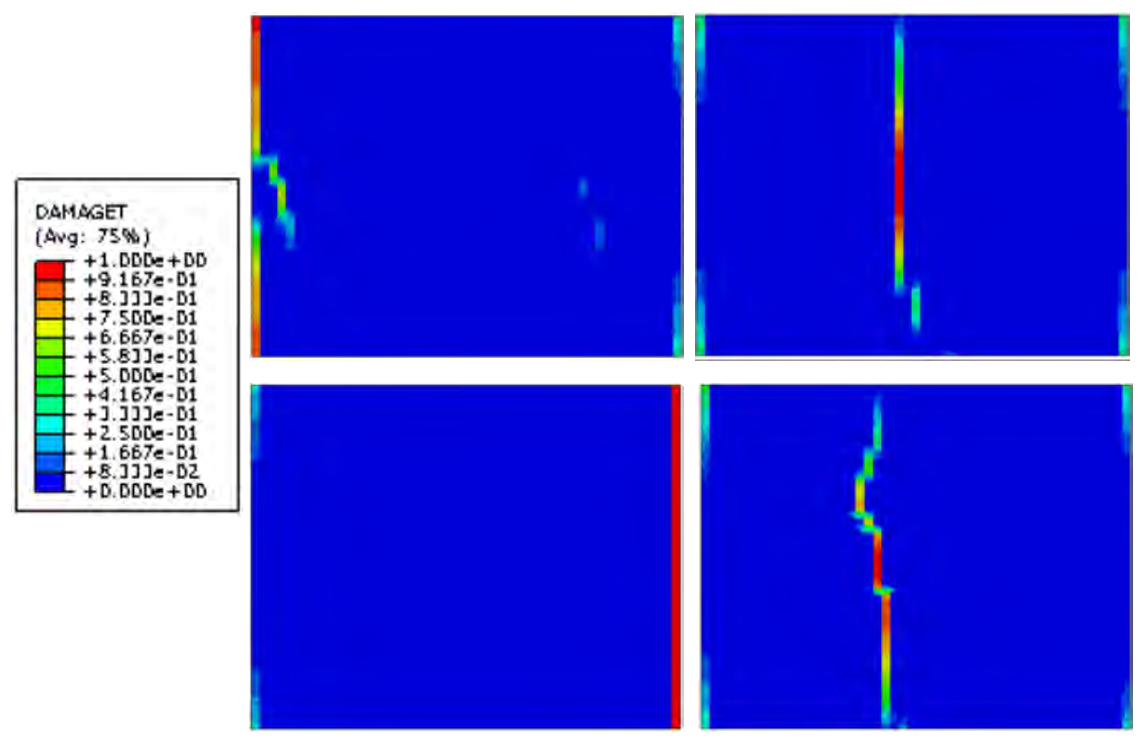

Figura 3.41: Variable de daño para la geometría de 50 x $50 \mathrm{~cm}$ biempotrada simulada con propiedades mecánicas aleatorias

luego crecer hacia el exterior (casos 2 y 9), a diferencia de lo que ocurre en las geometrías con relación de aspecto igual a 5 . 


\section{Capítulo 4}

\section{Programa experimental}

\subsection{Introducción}

En el presente capítulo se describe el programa experimental llevado a cabo para la caracterización de las propiedades físicas, térmicas y mecánicas del hormigón utilizado (tracción, compresión, módulo de elasticidad, energía de fractura, extensibilidad, contracción libre, contracción restringida, tiempo de fraguado y calor de hidratación) y para la validación del algoritmo desarrollado.

Es importante destacar que en el problema analizado es necesario conocer la evolución de las propiedades mecánicas del hormigón en función del tiempo. Por ello, tanto la resistencia a tracción como la resistencia a compresión y el módulo de elasticidad, se midieron a diferentes edades con el objetivo de obtener curvas de ajuste en las que se determina la variación de las mismas con el tiempo.

Los ensayos realizados se agruparon en tres etapas: etapa preliminar, ensayos de caracterización y ensayos de validación.

En la etapa preliminar se estudiaron tres hormigones para el ensayo de contracción restringida en anillos de hormigón ASTM C1581, variando el contenido de cemento en cada una de ellos.

En la siguiente etapa se llevó a cabo la caracterización en el tiempo de las propiedades mecánicas de la mezcla seleccionada en la etapa preliminar, determinando la resistencia a tracción, compresión y módulo de elasticidad a las edades de 1, 7 y 28 días. Se midió el módulo de elasticidad dinámico a diferentes edades y se realizaron ensayos de extensibilidad en vigas con la finalidad de considerar el efecto del creep en el ensayo de contracción restringida. Finalmente se moldearon probetas para el ensayo de contracción libre y para el ensayo de energía de fractura.

Para la etapa de validación experimental del algoritmo desarrollado se moldearon diez anillos de contracción restringida, tres de los cuales incluyeron entallas con el objetivo de favorecer la localización de las fisuras. 
En todos los ensayos el curado se llevó a cabo en cámara seca a $20^{\circ} \mathrm{C}$ y $60 \%$ de humedad relativa.

\subsection{Etapa preliminar}

En esta etapa se realizaron tres hormigones con distinta relación agua/cemento: $0.57,0.45$ y 0.39 . Las mezclas se diseñaron considerando un contenido de cemento estándar $\left(400 \mathrm{Kg} / \mathrm{m}^{3}\right)$ y dos contenidos de cemento más altos $\left(500\right.$ y $\left.600 \mathrm{Kg} / \mathrm{m}^{3}\right)$ con el objetivo de favorecer la aparición de fisuras a edades tempranas en el ensayo de contracción restringida en anillos de hormigón.

En la Tabla 4.1 se indica la dosificación de los tres hormigones. Para el mezclado fue utilizada una mezcladora de eje basculante de capacidad 150 1. En la Tabla 4.2 se muestran las propiedades en estado fresco de cada uno de ellos.

\begin{tabular}{|l|c|c|c|}
\hline \multicolumn{1}{|c|}{ Componente } & Hormigón 1 & Hormigón 2 & Hormigón 3 \\
\hline $\begin{array}{l}\text { Cemento CPN40 } \\
{\left[\mathrm{Kg} / \mathrm{m}^{3}\right]}\end{array}$ & 400 & 500 & 600 \\
\hline Agua $\left[\mathrm{Kg} / \mathrm{m}^{3}\right]$ & 226 & 226 & 232 \\
\hline $\begin{array}{l}\text { Arena silícea na- } \\
\text { tural }\left[\mathrm{Kg} / \mathrm{m}^{3}\right]\end{array}$ & 900 & 800 & 700 \\
\hline $\begin{array}{l}\text { Piedra partida } \\
\text { granítica (ta- } \\
\text { maño máximo } \\
12 \mathrm{~mm})\left[\mathrm{Kg} / \mathrm{m}^{3}\right]\end{array}$ & 800 & 800 & 800 \\
\hline
\end{tabular}

Tabla 4.1: Dosificaciones de los hormigones de la etapa preliminar

\begin{tabular}{|l|c|c|c|}
\hline \multicolumn{1}{|c|}{ Propiedad } & Hormigón 1 & Hormigón 2 & Hormigón 3 \\
\hline $\begin{array}{l}\text { Asentamiento } \\
\text { [cm] }\end{array}$ & 7 & 7.5 & 6.5 \\
\hline PUV [Kg/m3] & 2270 & 2330 & 2000 \\
\hline Aire [\%] & 3.5 & 1.9 & 5 \\
\hline
\end{tabular}

Tabla 4.2: Propiedades en estado fresco de los hormigones analizados en la etapa preliminar

Adicionalmente se realizaron ensayos de caracterización (compresión, tracción por compresión diametral y módulo de elasticidad) a la edad en la que se observaron fisuras en los anillos de contracción restringida moldeados con cada uno de los pastones. 
En la Figura 4.1 se observan imágenes del proceso de hormigonado y llenado de las probetas.
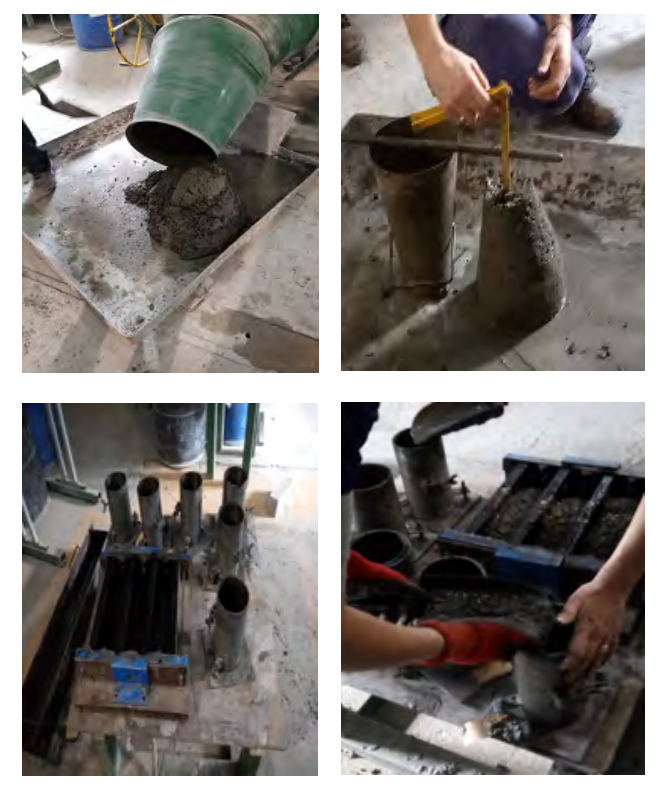

Figura 4.1: Proceso de hormigonado y llenado de probetas

\section{Ensayo de contracción restringida en anillos de hormigón- ASTM C1581}

Se han desarrollado diferentes procedimientos para estudiar la influencia de las propiedades del material en las deformaciones por contracción restringida, ejemplos de ello son el ensayo de barra, Parilee et al. (1989); Kovler (1994), el ensayo de plato/barra, Kraai (1985); Shales y Hover (1988) y los ensayos en anillos, Carlson y Reading (1998); Grzybowski y Shah (1990). El problema que presentan los ensayos de barra y de plato/barra es la dificultad de generar suficiente restricción, Weiss (1999).

Debido a su simplicidad y bajo costo el ensayo de contracción restringida en anillos ha sido el más utilizado para evaluar la fisuración por contracción en hormigón, Weiss et al. (2000, 2002); Moon et al. (2006). Este ensayo permite determinar la edad de fisuración y las tensiones de tracción desarrolladas en una probeta de mortero u hormigón bajo contracción restringida. El mismo consiste en moldear una muestra de hormigón o mortero en un molde circular alrededor de un anillo de acero. Para reducir la adherencia entre el acero y el hormigón se aplica una capa de aceite o grasa en la cara exterior del anillo de acero, al igual que en la base de apoyo.

AASHTO normalizó el ensayo recomendando la utilización de anillos de hormigón de $75 \mathrm{~mm}$ de espesor y anillos de acero de $12.5 \mathrm{~mm}$ de espesor, y 
también lo hizo ASTM bajo la designación C 1581/C 1581M-09, donde se recomienda la utilización de anillos de hormigón de $37.5 \mathrm{~mm}$ de espesor y anillos de acero de $12.5 \mathrm{~mm}$ de espesor. El problema que presentan los anillos de AASHTO es la baja restricción generada por el anillo de acero, por lo que en este caso los anillos de hormigón tardan más en fisurarse, Attiogbe et al. (2003). Otro problema que presentan es la existencia de gradientes de humedad debido al secado en dirección circunferencial, Dong et al. (2016); Hossain y Weiss (2006). La metodología propuesta por ASTM resuelve este inconveniente al utilizar anillos de menor espesor, pero no permite ensayar hormigones con tamaño de agregados grandes, Moon et al. (2006).

En la Figura 4.2 puede observarse el molde y el anillo de acero (izquierda) y la probeta (derecha) utilizados en el ensayo.

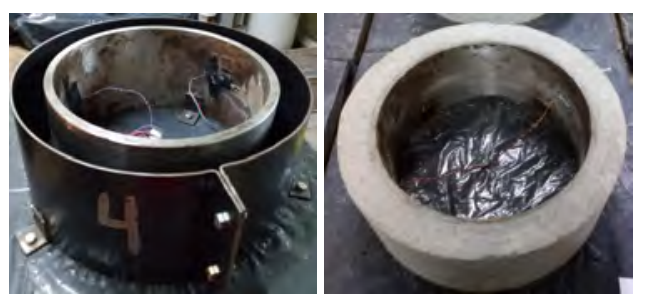

Figura 4.2: Ensayo de anillo de contracción restringida según norma ASTM C1581. Molde y anillo de acero (izquierda) probeta (derecha)

Las deformaciones por compresión en el anillo de acero, causadas por la contracción restringida en el hormigón, se miden utilizando galgas extensométricas (strain gages). Al momento de aparición de una fisura se genera una reducción repentina en el registro de las deformaciones por compresión en el anillo de acero. Las galgas extensométricas se encuentran ubicadas a la mitad de la altura en el interior del anillo en diámetros opuestos, conectadas cada una de ellas según una configuración de medio puente de Wheatstone. La mencionada orientación de las galgas permite la medición de las deformaciones en dirección circunferencial, ASTM (2004a).

El registro de las deformaciones se llevó a cabo mediante un módulo universal NI 9219 de cuatro canales para la medición de señales emitidas por galgas extensométricas, RTDs, termocuplas, celdas de carga y otros sensores de potencia. El equipo puede observarse en la Figura 4.3, conectado a los anillos. El módulo de medición se conecta a una computadora para realizar el registro continuo de las mediciones en intervalos de 15 minutos.

El ensayo de contracción restringida en anillos permite la comparación de mezclas pero no puede utilizarse para determinar la edad de fisuración de un hormigón o mortero en una estructura real, con una determinada configuración o tipo de exposición, ASTM (2004a).

Algunos autores proponen la utilización de anillos de geometría elíptica, Dong et al. $(2014,2016,2017)$ o anillos circulares excéntricos, Zhu et al. 


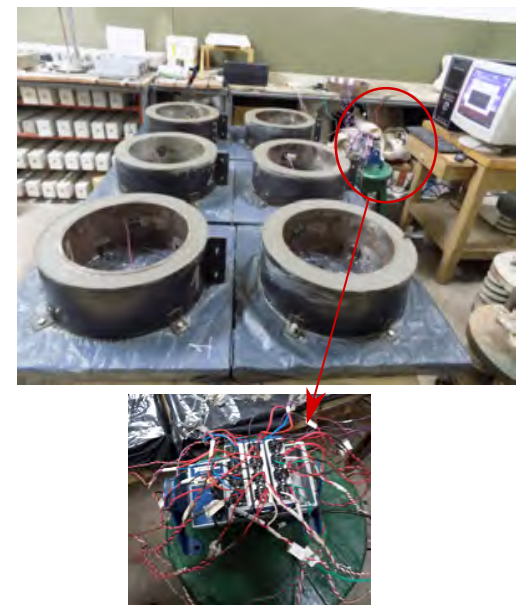

Figura 4.3: Equipo de registro de deformaciones para el ensayo de contracción restringida conectado a los anillos según norma ASTM C1581

(2015), con la finalidad de acelerar el tiempo de aparición de la primera fisura, ya que en este tipo de geometrías existen zonas en donde se producen concentraciones de tensiones.

Para cada uno de los hormigones presentados en la Tabla 4.1 se moldearon dos anillos de $406 \mathrm{~mm}$ de diámetro externo, $330 \mathrm{~mm}$ de diámetro interno y $150 \mathrm{~mm}$ de altura, ASTM (2004a).

\section{Resultados}

La fisura aparece en los puntos en que se observa un salto o discontinuidad en el gráfico de los registros de las deformaciones, las cuales representan una descompresión en el anillo de acero. A partir de ello pueden obtenerse los tiempos de aparición de las fisuras indicados en la Tabla 4.3 como diferencia entre el momento en que se produjo el salto y el momento en que se inició el ensayo.

\begin{tabular}{|c|c|}
\hline Pastón & Edad de fisuración [Días] \\
\hline Hormigón 1 & 10.04 \\
\hline Hormigón 2 & 9.13 \\
\hline Hormigón 3 & 7.63 \\
\hline
\end{tabular}

Tabla 4.3: Edad de fisuración de los anillos analizados en la etapa preliminar

En la Figura 4.4, se presentan las curvas representativas de los registros de deformaciones para cada uno de los pastones analizados. La letra " $F$ " y la flecha indican el salto correspondiente al momento de aparición de la fisura.

Para las siguientes etapas se adoptó el hormigón que fisuró más rápida- 


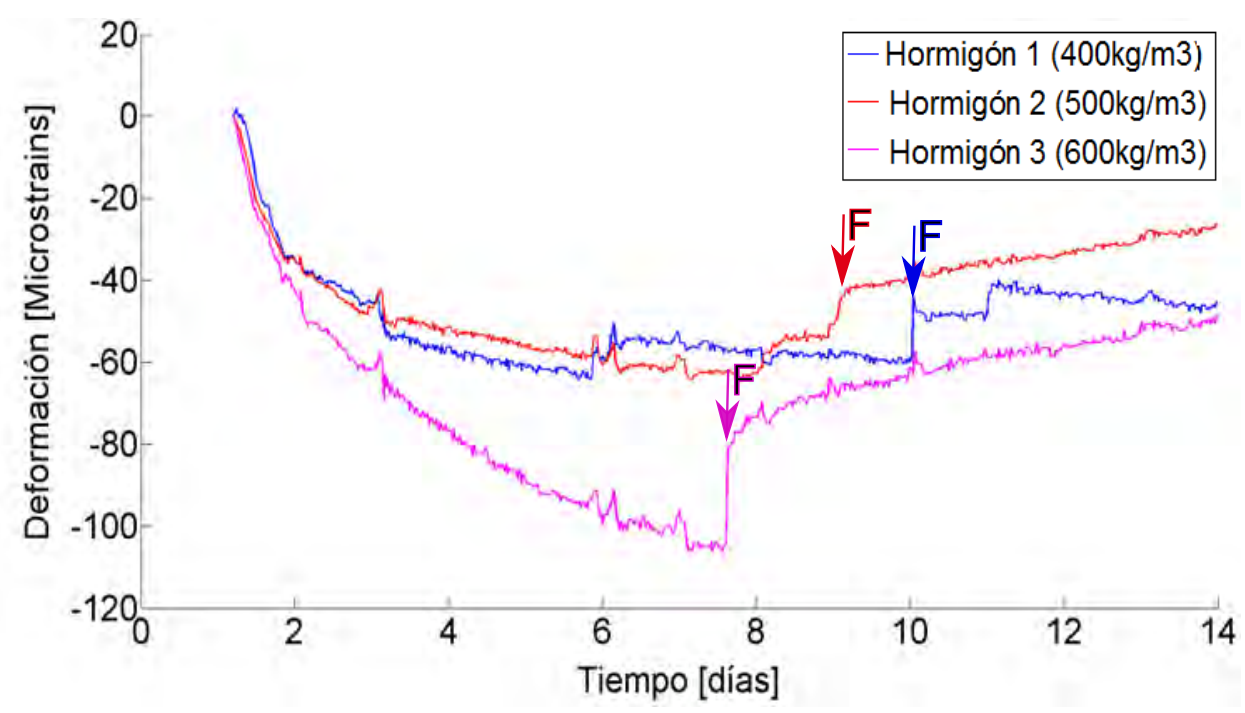

Figura 4.4: Registros de deformaciones para los anillos moldeados con el hormigón 1 (400 Kg/m $m^{3}$ de cemento), el hormigón 2 ( $500 \mathrm{Kg} / \mathrm{m}^{3}$ de cemento) y el hormigón $3\left(600 \mathrm{Kg} / \mathrm{m}^{3}\right.$ de cemento) en la etapa preliminar

mente con el objetivo de reducir los tiempos de ensayo, a pesar de que entre los hormigones analizados no se presentan diferencias significativas en las edades de fisuración, como se observa en la Tabla 4.3. Como era de esperarse este hormigón corresponde a la mezcla con el contenido de cemento más alto, en adelante denominado hormigón de $600 \mathrm{Kg}$ de cemento por $\mathrm{m}^{3}$.

\section{Ensayos de caracterización}

En las Tablas 4.4, 4.5 y 4.6 se presentan los resultados de resistencia a compresión, resistencia a tracción por compresión diametral y módulo de elasticidad para cada uno de los hormigones estudiados.

\begin{tabular}{|l|l|l|l|l|l|}
\hline \multicolumn{2}{|c|}{ Hormigón 1 } & \multicolumn{2}{c|}{ Hormigón 2 } & \multicolumn{2}{c|}{ Hormigón 3 } \\
\hline Probeta & $\begin{array}{l}f_{c} \\
{[\mathrm{MPa}]}\end{array}$ & Probeta & $\begin{array}{l}f_{c} \\
{[\mathrm{MPa}]}\end{array}$ & Probeta & $\begin{array}{l}f_{c} \\
{[\mathrm{MPa}]}\end{array}$ \\
\hline B14 & 26.74 & $\mathrm{~A} 4$ & 41 & $\mathrm{C} 26$ & 46.35 \\
\hline B16 & 36.29 & $\mathrm{~A} 5$ & 41.38 & $\mathrm{C} 27$ & 44.56 \\
\hline B17 & 29.92 & $\mathrm{~A} 7$ & 39.09 & $\mathrm{C} 29$ & 45.84 \\
\hline Promedio & 30.98 & & 40.49 & & 45.58 \\
\hline
\end{tabular}

Tabla 4.4: Resultados ensayos de resistencia a la compresión de los hormigones 1,2 y 3 a la edad de fisuración en la etapa preliminar 


\begin{tabular}{|l|l|l|l|l|l|}
\hline \multicolumn{2}{|c|}{ Hormigón 1 } & \multicolumn{2}{c|}{ Hormigón 2 } & \multicolumn{2}{c|}{ Hormigón 3 } \\
\hline Probeta & $\begin{array}{l}f_{c t} \\
{[\mathrm{MPa}]}\end{array}$ & Probeta & $\begin{array}{l}f_{c t} \\
{[\mathrm{MPa}]}\end{array}$ & Probeta & $\begin{array}{l}f_{c t} \\
{[\mathrm{MPa}]}\end{array}$ \\
\hline B13 & 2.08 & $\mathrm{~A} 6$ & 3.28 & $\mathrm{C} 24$ & 2.97 \\
\hline B15 & 2.43 & $\mathrm{~A} 8$ & 3.33 & $\mathrm{C} 25$ & 3.12 \\
\hline B18 & 2.36 & $\mathrm{~A} 9$ & 2.32 & $\mathrm{C} 28$ & 2.97 \\
\hline Promedio & 2.29 & & 2.98 & & 3.02 \\
\hline
\end{tabular}

Tabla 4.5: Resultados ensayos de resistencia a la tracción por compresión diametral de los hormigones 1, 2 y 3 a la edad de fisuración en la etapa preliminar

\begin{tabular}{|c|c|c|c|c|c|}
\hline \multicolumn{2}{|c|}{ Hormigón 1 } & \multicolumn{2}{c|}{ Hormigón 2 } & \multicolumn{2}{c|}{ Hormigón 3 } \\
\hline Probeta & E [MPa] & Probeta & E [MPa] & Probeta & E [MPa] \\
\hline B14 & 18532 & A4 & 26729 & C26 & 23341 \\
\hline B16 & 25589 & A5 & 25530 & C27 & 31651 \\
\hline B17 & 19497 & A7 & 22941 & C29 & 30249 \\
\hline Promedio & 21206 & & 25067 & & 28414 \\
\hline
\end{tabular}

Tabla 4.6: Resultados ensayos de módulo de elasticidad estático de los hormigones 1, 2 y 3 a la edad de fisuración en la etapa preliminar

\subsection{Ensayos de caracterización}

\subsubsection{Tiempo de fraguado}

Para la realización de este ensayo se tamiza una muestra de hormigón utilizando un tamiz IRAM 4.75, para separar el agregado grueso del mortero y se coloca este último en un recipiente cilíndrico de $20 \mathrm{~cm}$ de diámetro y $9.5 \mathrm{~cm}$ de altura, llevando a cabo su compactación en forma manual. La superficie del mortero debe estar a no menos de $10 \mathrm{~mm}$ debajo del borde del recipiente, para que sea posible extraer el agua de exudación con una pipeta, IRAM-1622 (1995). Para evitar la excesiva evaporación de agua se debe mantener la superficie de la muestra cubierta, excepto cuando se determina la penetración.

El equipo de ensayo utilizado se denomina penetrómetro, el cual permite la aplicación de una fuerza de hasta $500 \mathrm{~N}$. La menor división de la escala será menor o igual a $10 \mathrm{~N}$ o una deformación equivalente a $10 \mathrm{~N}$, cuando se trate de un aro dinamométrico. El aparato está provisto de un vástago con un resalto lateral que indica la penetración correspondiente a $25 \mathrm{~mm}$ y que permite el acoplamiento de las agujas que se utilizan para medir la penetración en su parte inferior. Estas agujas son removibles, y se denominan de acuerdo a su área, medida en milímetros cuadrados: 645, 323, 161, 65, 32, 
y 16, IRAM-1622 (1995). En la Figura 4.5 se observa el equipo y la muestra utilizada para el ensayo.
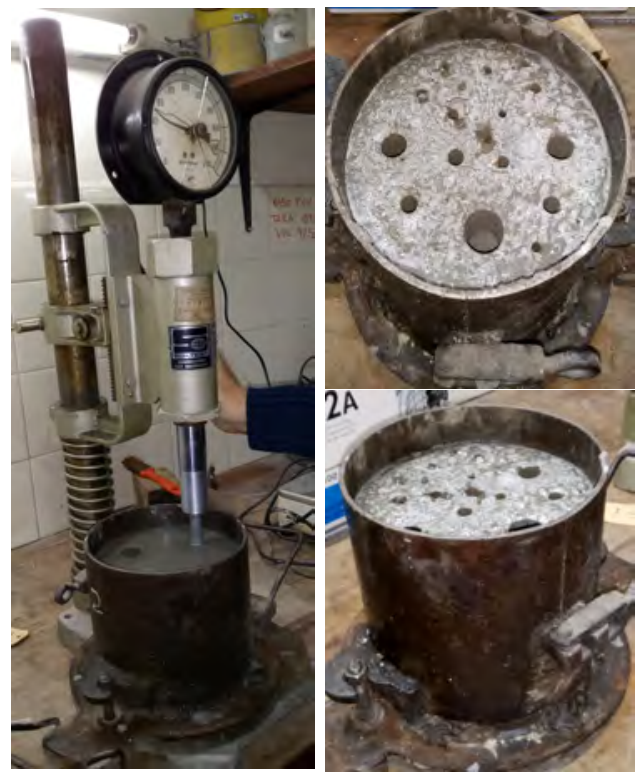

Figura 4.5: Ensayo para la determinación del tiempo de fraguado para un hormigón con un contenido de cemento de $600 \mathrm{Kg} / \mathrm{m}^{3}$

Una vez retirada el agua de exudación de la muestra, se selecciona una aguja, comenzando por las de mayor superficie, y se coloca la misma en el penetrómetro. Luego la superficie inferior de la aguja se pone en contacto con la superficie del mortero, y se aplica una fuerza vertical de arriba hacia abajo con el aparato hasta que la aguja penetre $25 \mathrm{~mm}$ en la superficie del mortero, lo que puede verificarse a través del resalto lateral en el vástago del equipamiento. Se registra la fuerza necesaria y el tiempo transcurrido a partir del contacto de la aguja con el mortero.

La distancia entre las impresiones de la aguja será por lo menos igual a dos diámetros de la aguja utilizada, no debiendo ser menor que $15 \mathrm{~mm}$. La distancia entre cualquier impresión de la aguja y los costados del recipiente debe ser igual o mayor que $25 \mathrm{~mm}$, IRAM-1622 (1995).

La determinación de la profundidad de penetración de la aguja debe realizarse a intervalos regulares de tiempo, para obtener una curva uniforme. En este caso se realizaron mediciones cada media hora. A medida que el hormigón se endurece se utilizan agujas de menor área.

La resistencia a la penetración se obtiene como el cociente entre la fuerza necesaria para producir la penetración y el área de la cara inferior de la aguja. Durante el ensayo se calcula la resistencia a la penetración correspondiente a cada intervalo de tiempo transcurrido y se grafica dicha relación. El tiempo de fraguado inicial será aquel para el cual se alcance una resis- 
tencia a la penetración de $35 \mathrm{Kg} / \mathrm{cm}^{2}$ y el tiempo final de fraguado será el correspondiente a una resistencia a la penetración de $276 \mathrm{Kg} / \mathrm{cm}^{2}$.

\section{Resultados}

En la Figura 4.6 se presenta la curva correspondiente a la determinación del tiempo de fraguado. Puede observarse que el fraguado inicial (TFI) tiene lugar a las $4.8 \mathrm{hs}$ y el fraguado final (TFF) a las $7 \mathrm{hs}$.

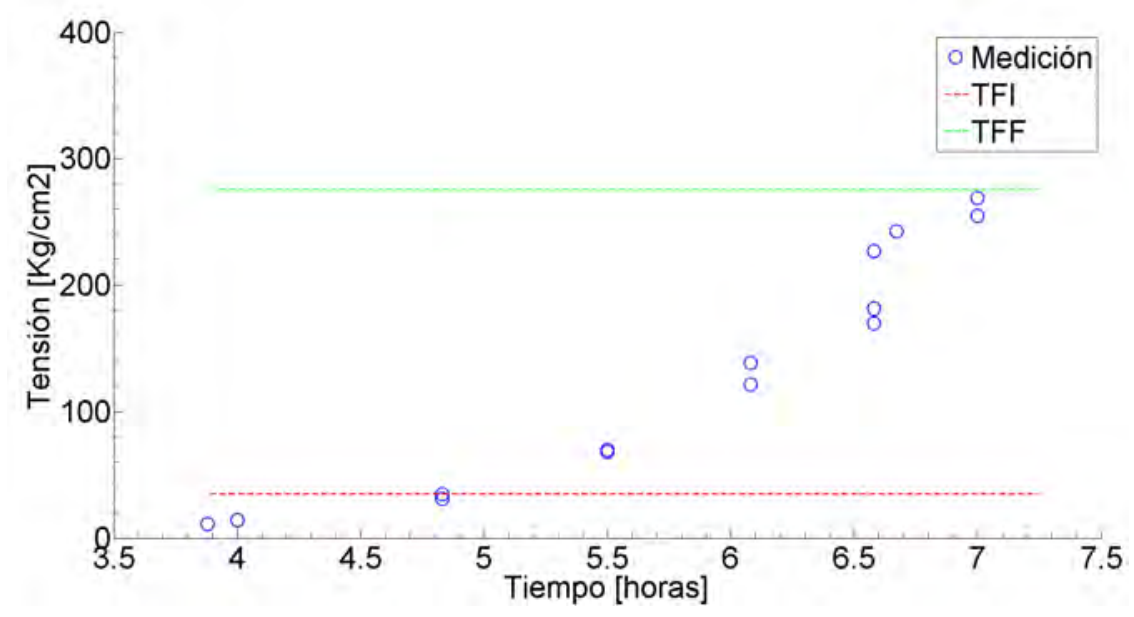

Figura 4.6: Medición tiempo de fraguado para el hormigón de $600 \mathrm{Kg} / \mathrm{m}^{3}$ de cemento

\subsubsection{Curva de elevación adiabática de la temperatura del hormigón}

La curva de elevación adiabática representa la temperatura alcanzada por la masa de hormigón, desde su colocación en estado fresco hasta después de endurecido, sin que se produzca intercambio de calor con el medio que la rodea. Para determinarla se colocó una muestra de hormigón de aproximadamente $800 \mathrm{~g}$ en un recipiente de telgopor con tapa, en cámara seca (HR:60 \%, T:20 ${ }^{\circ} \mathrm{C}$ ). A través de un agujero practicado en la tapa de dicho recipiente, se introdujo una termocupla tipo $\mathrm{J}$ (hierro-constatan) conectada a un registrador Novous, ver Figura 4.7, con el objetivo de obtener en forma continua la temperatura. Además se colocó otra termocupla que registra la temperatura ambiente. 


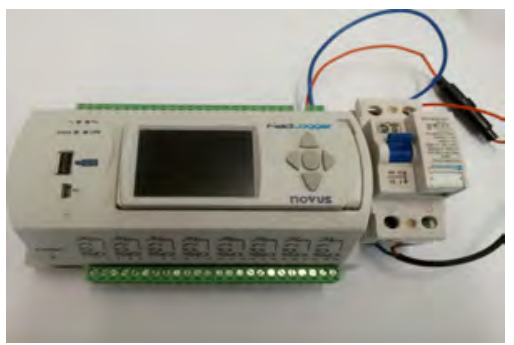

Figura 4.7: Registrador de temperatura

\section{Resultados}

En la Figura 4.8 se presenta la curva de elevación adiabática del hormigón estudiado.

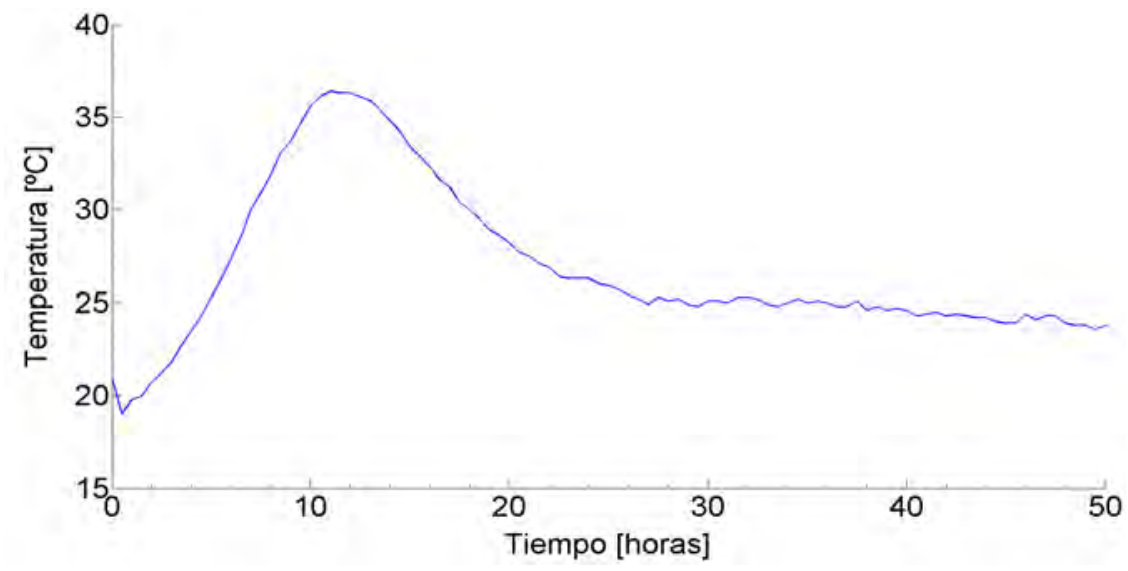

Figura 4.8: Curva de elevación adiabática para el hormigón estudiado

\subsubsection{Resistencia a la compresión}

Se moldearon nueve cilindros de $10 \mathrm{~cm}$ de diámetro y $20 \mathrm{~cm}$ de altura, elaborados según lo establecido por la norma IRAM-1524 (1982), compactados con vibración externa a través de mesa vibradora y curados en cámara seca (HR: $\left.60 \%, \mathrm{~T}: 20^{\circ} \mathrm{C}\right)$. Para la determinación de la resistencia a compresión se empleó una prensa AMSLER de $2000 \mathrm{KN}$.

Este ensayo consiste en aplicar una carga axial de compresión a una velocidad tal que genere en las probetas una tasa de tensión de $0.25 \pm 0.05$ $\mathrm{MPa} / \mathrm{seg}$ hasta la rotura. La resistencia a compresión se determina dividiendo el valor de la máxima carga obtenida durante el ensayo por la sección transversal de la probeta, ASTM (2004b). Para las probetas se utilizó un encabezado de neopreno.

Los ensayos fueron llevados a cabo a las edades de 1, 7 y 28 días utilizando 
tres probetas en cada una de las edades. En la Figura 4.9 se observa la máquina de ensayo y la probeta ensayada.

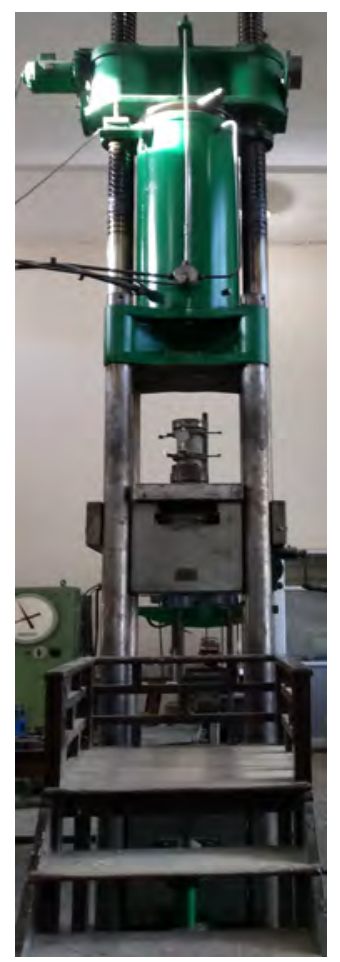

Figura 4.9: Ensayo de compresión en probetas cilíndricas de 10 x $20 \mathrm{~cm}$

\section{Resultados}

En la Tabla 4.7 se presentan los resultados de los ensayos de resistencia a compresión.

\begin{tabular}{|c|c|c|c|c|c|}
\hline \multicolumn{2}{|c|}{ Edad 1 día } & \multicolumn{2}{c|}{ Edad 7 días } & \multicolumn{2}{c|}{ Edad 28 días } \\
\hline Probeta & $f_{c}[\mathrm{MPa}]$ & Probeta & $f_{c}[\mathrm{MPa}]$ & Probeta & $f_{c}[\mathrm{MPa}]$ \\
\hline PA7 & 16.89 & PA13 & 39.62 & PA20 & 48.90 \\
\hline PA10 & 14.89 & PA16 & 41.06 & PA27 & 46.99 \\
\hline PA21 & 16.57 & PA18 & 40.00 & PA30 & 49.14 \\
\hline Promedio & 16.12 & & 40.23 & & 48.34 \\
\hline
\end{tabular}

Tabla 4.7: Resultados de los ensayos de resistencia a la compresión en probetas cilíndricas de $10 \times 20 \mathrm{~cm}$

A partir de los valores promedio de la resistencia a compresión a cada una de las edades ensayadas se obtuvo la curva de ajuste presentada en la 
Figura 4.10 correspondiente a la ecuación indicada a continuación:

$$
f_{c}(t)=f_{c 28} * e^{\left(0,25 *\left(1-\left(\frac{28}{t}\right)^{0,5}\right)\right)}
$$

donde $f_{c 28}$ es la resistencia a compresión a 28 días y el tiempo se mide en días.

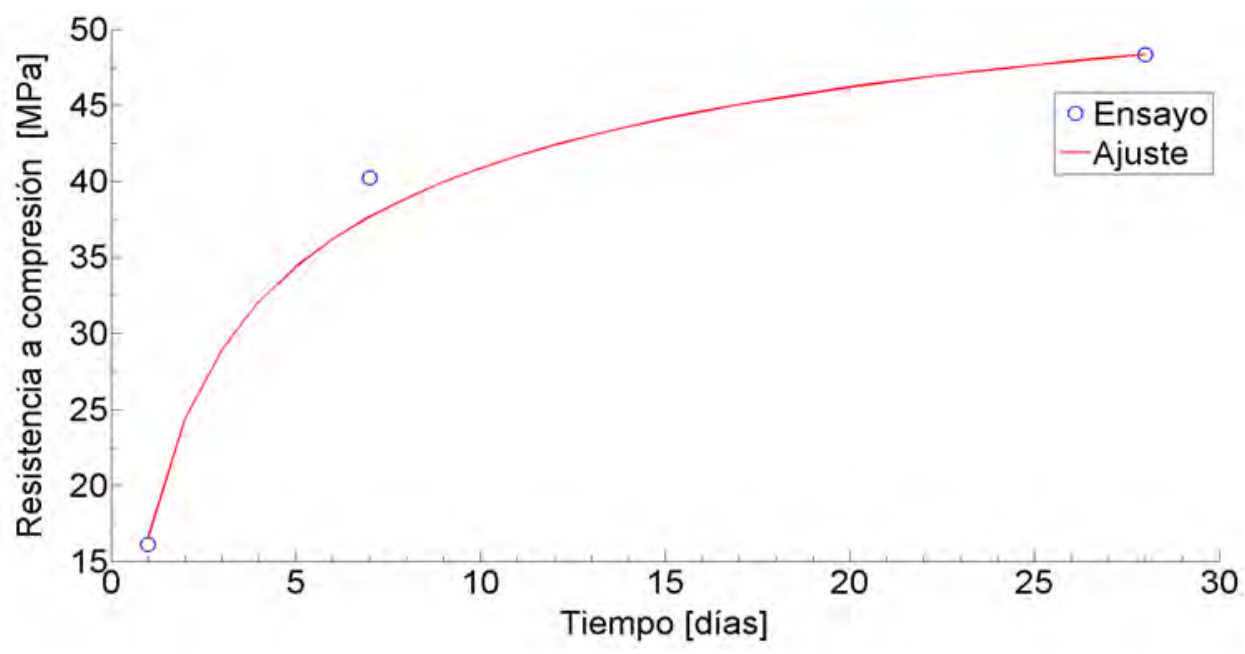

Figura 4.10: Evolución de la resistencia a compresión en el tiempo y curva de ajuste

\subsubsection{Resistencia a la tracción por compresión diametral}

Se moldearon nueve probetas cilíndricas de $10 \mathrm{~cm}$ de diámetro y $20 \mathrm{~cm}$ de altura, elaboradas según lo establecido por la norma IRAM-1524 (1982), compactadas con vibración externa a través de mesa vibradora y curadas en cámara seca (HR:60\%, T:20 ${ }^{\circ} \mathrm{C}$ ). Para la determinación de la resistencia a tracción por compresión diametral se empleó una prensa AMSLER (capacidad $300 \mathrm{KN})$.

Este ensayo consiste en la aplicación de una carga linealmente distribuida a lo largo de las dos generatrices opuestas de una probeta cilíndrica de hormigón, como se esquematiza en la Figura 4.11, con una velocidad de carga tal que permita el desarrollo de una tensión de tracción por compresión diametral de 0.7 a $1.4 \mathrm{MPa} / \mathrm{min}$ hasta la rotura de la probeta. Esta carga genera tensiones de tracción en el plano en que se encuentra contenida la carga aplicada. En lugar de una falla por compresión tendrá lugar una falla por tracción debido a que las áreas en las que se aplica la carga se encuentran bajo un estado de compresión triaxial, resistiendo tensiones de compresión más altas que las indicadas en un ensayo de compresión uniaxial. Para dis- 
tribuir la carga aplicada a lo largo de la longitud del cilindro se utiliza una faja de madera ASTM (2004c).

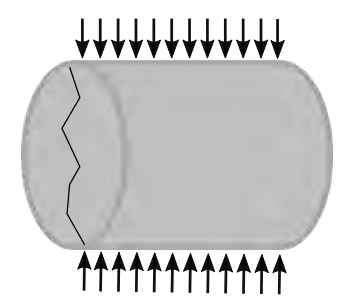

Figura 4.11: Aplicación de cargas en el ensayo de tracción por compresión diametral

El valor de la resistencia a tracción se calcula a partir de la siguiente expresión, ASTM (2004c):

$$
f_{c t}=\frac{2 P}{\pi l d}
$$

donde $P$ es la carga máxima, $l$ es la longitud de la probeta y $d$ el diámetro de la misma.

La resistencia a tracción obtenida con este método es generalmente mayor que la resultante de ensayos de tracción directa y menor que la obtenida a partir de módulo de rotura.

Los ensayos fueron llevados a cabo a las edades de 1, 7 y 28 días, utilizando tres probetas en cada una de las edades. En la Figura 4.12 se observa la máquina de ensayo y la probeta ensayada.

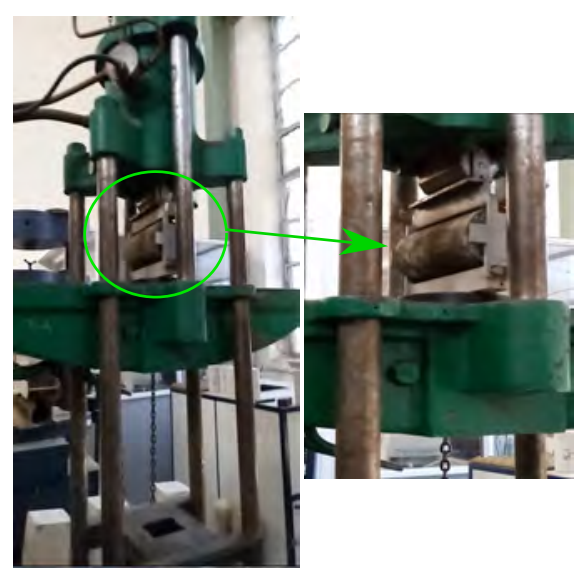

Figura 4.12: Ensayo de tracción por compresión diametral en probetas cilíndricas de $10 \times 20 \mathrm{~cm}$ 


\section{Resultados}

En la Tabla 4.8 se presentan los resultados de los ensayos de resistencia a tración por compresión diametral a las edades de 1, 7 y 28 días

\begin{tabular}{|c|c|c|c|c|c|}
\hline \multicolumn{2}{|c|}{ Edad 1 día } & \multicolumn{2}{c|}{ Edad 7 días } & \multicolumn{2}{c|}{ Edad 28 días } \\
\hline Probeta & $f_{c t}[\mathrm{MPa}]$ & Probeta & $f_{c t}[\mathrm{MPa}]$ & Probeta & $f_{c t}[\mathrm{MPa}]$ \\
\hline PA9 & 1.82 & PA24 & 3.52 & PA15 & 3.55 \\
\hline PA14 & 1.96 & PA26 & 3.14 & PA22 & 3.43 \\
\hline PA23 & 2.15 & PA29 & 3.51 & PA25 & 3.59 \\
\hline Promedio & 1.97 & & 3.39 & & 3.52 \\
\hline
\end{tabular}

Tabla 4.8: Resultados de los ensayos de resistencia a la tracción por compresión diametral

A partir de los valores promedio de la resistencia a tracción por compresión diametral a cada una de las edades ensayadas se obtuvo la curva de ajuste presentada en la Figura 4.13 correspondiente a la ecuación indicada a continuación:

$$
f_{c t}(t)=1,01 * f_{c t 28} * e^{\left(0,14 *\left(1-\left(\frac{28}{t}\right)^{0,49}\right)\right)}
$$

donde $f_{c t 28}$ es la resistencia a tracción a 28 días y el tiempo se mide en días.

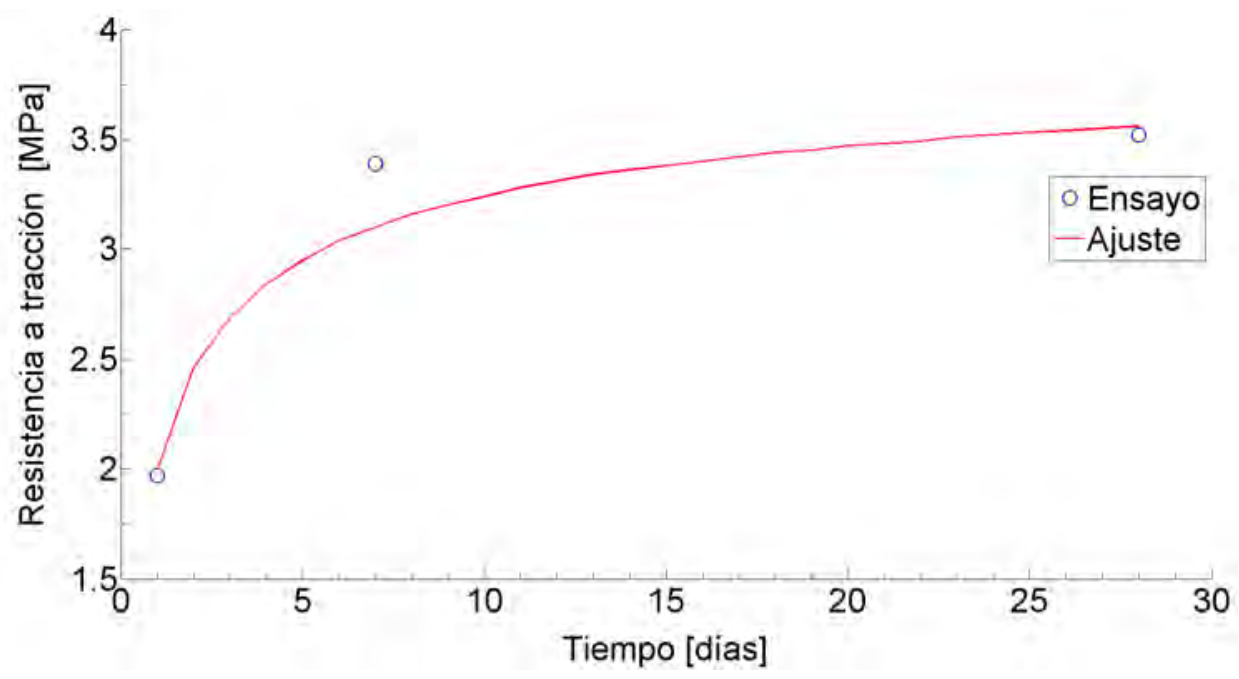

Figura 4.13: Evolución de la resistencia a tracción en el tiempo y curva de ajuste 


\subsubsection{Módulo de elasticidad estático}

Se moldearon siete cilindros de $10 \mathrm{~cm}$ de diámetro y $20 \mathrm{~cm}$ de altura, elaborados según lo establecido por la norma IRAM-1524 (1982), compactados con vibración externa a través de mesa vibratoria y curados en cámara seca (HR:60\%, T:20 ${ }^{\circ} \mathrm{C}$ ). Para la determinación del módulo de elasticidad se empleó una prensa AMSLER (capacidad $2000 \mathrm{KN}$ ).

Para obtener el módulo de elasticidad estático se utiliza un compresómetro como el indicado en la Figura 4.14. El mismo consiste en dos abrazaderas, una de ellas rígidamente unida a la probeta y la otra vinculada a la misma en dos puntos diametralmente opuestos, permitiendo la rotación libre de la misma. En un punto de esta última abrazadera, a media distancia entre los puntos de soporte, se emplea una barra pivote para mantener constante la distancia entre las dos abrazaderas. En el punto opuesto de la circunferencia de la abrazadera rotativa, el cambio en la distancia entre las abrazaderas es igual a la suma del desplazamiento debido a la deformación de la probeta y el desplazamiento debido a la rotación de la abrazadera alrededor del pivote ASTM-C-469-02 (2002).

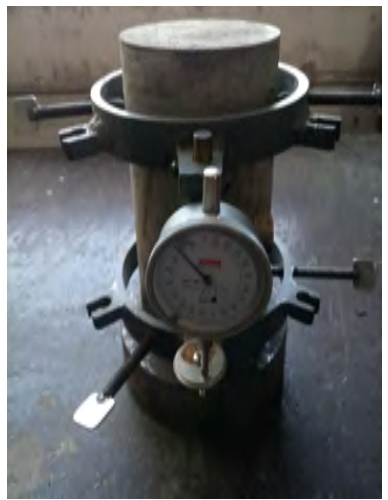

Figura 4.14: Compresómetro utilizado para el ensayo del módulo de elasticidad estático en probetas cilíndricas de $10 \times 20 \mathrm{~cm}$

La probeta se coloca con el compresómetro en la máquina de ensayo y se carga al menos dos veces, pero durante la primera carga no se realizan registros. La carga se aplica en forma constante a una velocidad tal que provoque una tensión de $241 \pm 34 \mathrm{KPa} / \mathrm{seg}$. Se registra el valor de la carga y la deformación longitudinal cuando esta última es $50 \times 10^{-6}$ y cuando la carga aplicada es $40 \%$ de la carga última.

El cálculo del módulo de elasticidad estático se realiza a partir de la expresión 4.4, ASTM-C-469-02 (2002):

$$
E=\left(\frac{\sigma_{2}-\sigma_{1}}{\epsilon_{2}-0,000050}\right)
$$

donde $E$ es el módulo de elasticidad en $\mathrm{MPa}, \sigma_{2}$ la tensión correspondiente 
al $40 \%$ de la carga última, $\sigma_{1}$ la tensión correspondiente a una deformación longitudinal de $50 \times 10^{-6}$ y $\epsilon_{2}$ la deformación longitudinal calculada para la tensión $\sigma_{2}$.

\section{Resultados}

En la Tabla 4.9 se presentan los resultados de los ensayos de módulo de elasticidad estático a las edades de 1,7 y 28 días

\begin{tabular}{|c|c|c|c|c|c|}
\hline \multicolumn{2}{|c|}{ Edad 1 día } & \multicolumn{2}{c|}{ Edad 7 días } & \multicolumn{2}{c|}{ Edad 28 días } \\
\hline Probeta & E $[\mathrm{MPa}]$ & Probeta & E $[\mathrm{MPa}]$ & Probeta & E [MPa] \\
\hline PA7 & 17372 & PA16 & 26068 & PA20 & 26556 \\
\hline PA21 & 20708 & PA18 & 28359 & PA27 & 27428 \\
\hline & & & & PA30 & 27992 \\
\hline Promedio & 19040 & & 27214 & & 27326 \\
\hline
\end{tabular}

Tabla 4.9: Resultados ensayos de módulo de elasticidad estático

A partir de los valores promedio del módulo de elasticidad estático a cada una de las edades ensayadas se obtuvo la curva de ajuste presentada en la Figura 4.15 correspondiente a la ecuación indicada a continuación:

$$
E(t)=E_{28} *\left(e^{\left(0,10 *\left(1-\left(\frac{28}{t}\right)^{0,50}\right)\right)}\right)^{0,50}
$$

donde $E_{28}$ es el módulo de elasticidad estático a 28 días y el tiempo se mide en días.

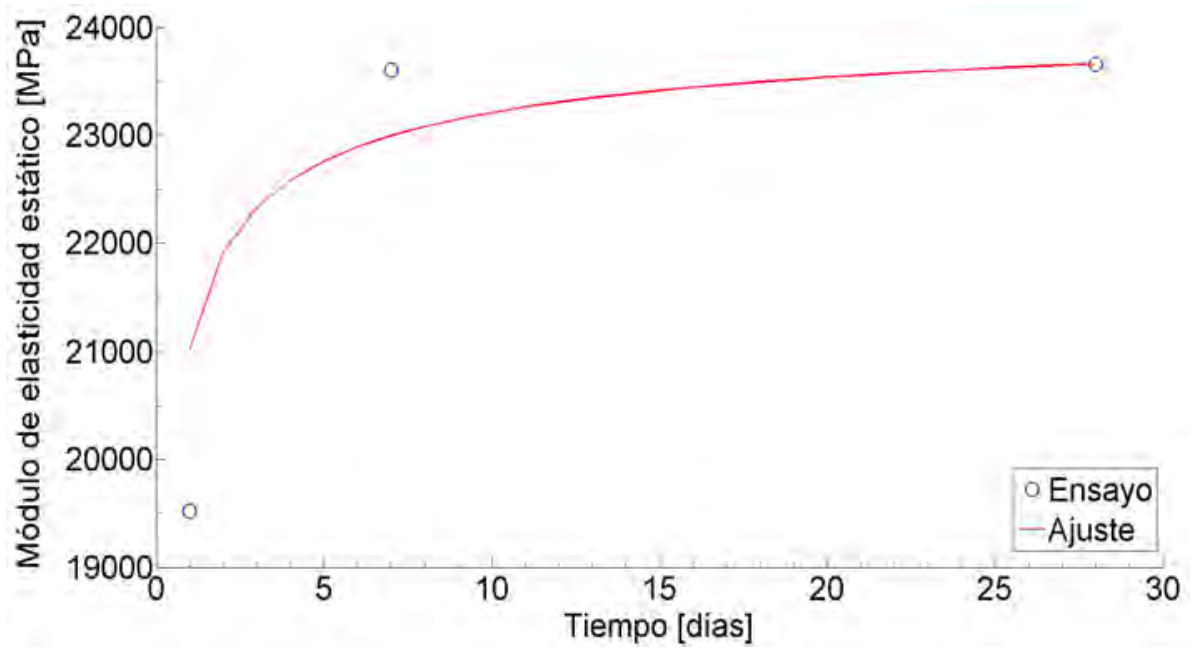

Figura 4.15: Evolución módulo de elasticidad estático en el tiempo y curva de ajuste 


\subsubsection{Módulo de elasticidad dinámico}

Se moldearon tres vigas de $7 \times 10.5 \times 43 \mathrm{~cm}$ según lo establecido por la norma IRAM-1524 (1982), compactadas con vibración externa a través de mesa vibratoria y curadas en cámara seca (HR:60\%, T:20 ${ }^{\circ} \mathrm{C}$ ). Para la determinación del módulo de elasticidad dinámico se utilizó un equipo sónico con un rango de frecuencia que oscila entre $10 \mathrm{KHz}$ y $100 \mathrm{KHz}$.

Este es un ensayo de tipo no destructivo, lo cual significa que no se produce la rotura de la probeta. El mismo consiste en medir las frecuencias de resonancia de las probetas excitándolas continuamente a frecuencias variables mediante el uso de un transductor que transforma una señal eléctrica cíclica en una fuerza mecánica cíclica sobre la probeta. Un segundo transductor percibe las vibraciones mecánicas resultantes de la probeta y las transforma en una señal eléctrica. La amplitud y la frecuencia de la señal se miden mediante osciloscopios para detectar resonancia. Las frecuencias de resonancia junto con las dimensiones y la masa de la probeta se utilizan para calcular el módulo de elasticidad dinámico, ASTM (2013b). En la Figura 4.16 se observa una imagen del ensayo.

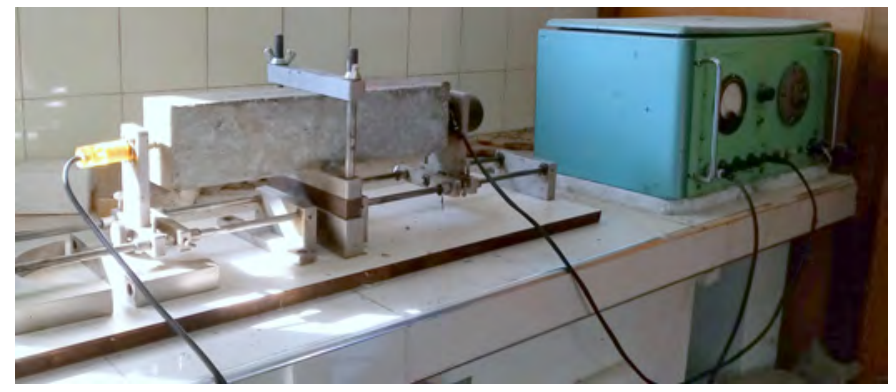

Figura 4.16: Ensayo para la determinación del módulo de elasticidad dinámico

El módulo de elasticidad dinámico para una probeta de sección rectangular se obtiene a partir de la expresión 4.6:

$$
\begin{aligned}
E_{d} & =e * n^{2} * P \\
e & =c t e * \frac{l}{b * h}
\end{aligned}
$$

donde, $P$ es el peso de la probeta, $l$ la longitud, $h$ la altura, $b$ el ancho y $n$ es un valor que se obtiene de tablas a partir de la frecuencia de resonancia.

Los ensayos fueron llevados a cabo a las edades de 1, 5, 7, 12 y 39 días.

\section{Resultados}

En la Tabla 4.10 se presentan los resultados de los ensayos de módulo de elasticidad dinámico a las edades de 1, 5, 7, 12 y 39 días 
CaPítulo 4. Programa experimental

\begin{tabular}{|c|c|c|c|c|c|}
\hline Probeta & Edad 1 días & Edad 5 días & Edad 7 días & Edad 12 días & Edad 39 días \\
\cline { 2 - 6 } & E $[\mathrm{MPa}]$ & E $[\mathrm{MPa}]$ & E [MPa] & E [MPa] & E [MPa] \\
\hline PA1 & 25462 & 33761 & 34290 & 34707 & 34050 \\
\hline PA4 & 25246 & 33858 & 35652 & 35203 & 36977 \\
\hline PA5 & 25211 & 34412 & 34857 & 35036 & 34843 \\
\hline Promedio & 25306 & 34011 & 34933 & 34982 & 35290 \\
\hline
\end{tabular}

Tabla 4.10: Resultados ensayos de módulo de elasticidad dinámico

A partir de los valores promedio del módulo de elasticidad dinámico a cada una de las edades ensayadas se obtuvo la curva de ajuste presentada en la Figura 4.17 correspondiente a la ecuación indicada a continuación

$$
E_{d}(t)=E_{d 28} *\left(e^{\left(0,10 *\left(1-\left(\frac{12}{t}\right)^{0,50}\right)\right)}\right)^{0,50}
$$

donde $E_{d 28}$ es el módulo de elasticidad dinámico a 28 días y el tiempo se mide en días.

Comparando las expresiones 4.5 y 4.8 se observa que los módulos de elas-

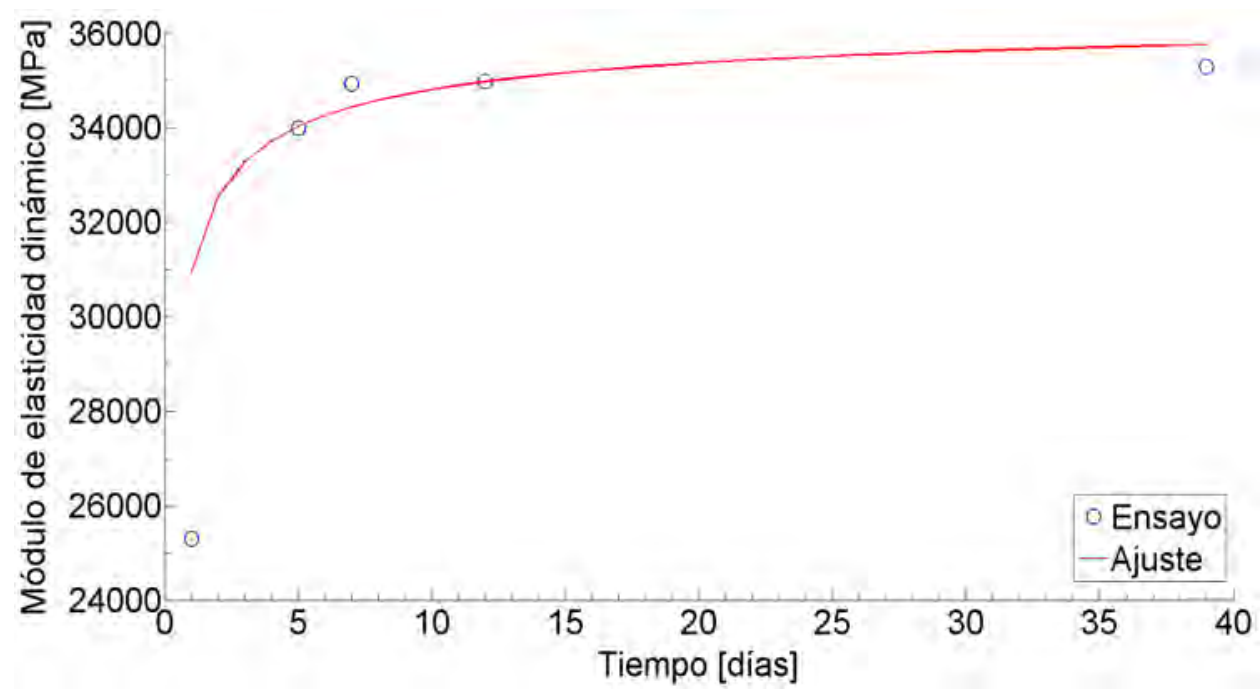

Figura 4.17: Evolución módulo de elasticidad dinámico en el tiempo y curva de ajuste

ticidad estático y dinámico siguen la misma ley de evolución en el tiempo. Como se explicó anteriormente el módulo de elasticidad dinámico es un ensayo no destructivo de fácil determinación, por lo que conociendo la evolución del mismo en el tiempo y el valor del módulo de elasticidad estático a una determinada edad, puede estimarse la evolución de este último. 


\subsubsection{Ensayo de contracción libre}

\section{Consideraciones preliminares}

Para llevar a cabo el modelo numérico es necesario determinar un estado de cargas que simule la contracción generada en condiciones de laboratorio. Puede considerarse que el cambio de longitud de prismas de hormigón bajo contracción libre es el mismo que experimentarían si fueran expuestos a una temperatura ficticia, Dong et al. (2016). Además, puede aceptarse que la deformación por contracción libre es la misma para elementos de hormigón con la misma relación área expuesta/volumen $(A / V)$, pero con diferente geometría, Dong et al. (2016). Esto último permite vincular el ensayo de contracción libre con el ensayo de contracción restringida en anillos, ya que para una geometría cilíndrica como la del anillo resulta dificultoso la medición de la contracción libre. Con el objetivo de obtener distintas relaciones $A / V$ se propone utilizar probetas prismáticas de $7 \times 7 \times 30 \mathrm{~cm}$ de contracción libre con distintas condiciones de aislación: todas las caras de la probeta pintadas, dos caras contiguas de la probeta libres, una cara de la probeta libre, todas las caras de la probeta libres, Dong et al. (2016). Para elegir el método de aislación más eficiente se probaron cuatro tipos de aislaciones:

- Emulsión asfáltica + papel de aluminio

- Pintura epoxi bituminosa SHER TAR 400 BR

- Membrana poliuretánica Sikalastic-560

- Silicona

Se saturaron 10 probetas de $7 \times 7 \times 15 \mathrm{~cm}$ en agua durante 72 horas, luego se pintaron las mismas con cada una de las pinturas mencionadas anteriormente y finalmente se colocaron en horno a $70^{\circ} \mathrm{C}$ para medir la pérdida de peso. Se dejaron dos probetas sin pintar, para utilizarlas como control. En la Figura 4.18 se observan las probetas utilizadas en el ensayo y el horno.

El objetivo de este ensayo es adoptar el aislante para el cual la probeta de hormigón presente la menor pérdida de peso. En la Figura 4.19 se muestran las curvas de pérdida de peso para las probetas con los diferentes aislantes utilizados. Se observa la menor pérdida de peso en las probetas que fueron pintadas con la emulsión asfáltica, por lo que se utilizará dicha pintura para aislar las probetas utilizadas en el ensayo de contracción libre.

Es importante mencionar que si bien se utilizó la pérdida de peso como criterio para la selección de la aislación a utilizar, que no se registre pérdida de peso no implica que no se produzca contracción, ya que lo que determina que ocurra el fenómeno de contracción es la formación de una interfase entre el agua y el aire denominada menisco. Por la interacción del menisco con las paredes de los poros el agua se encuentra sometida a una presión 

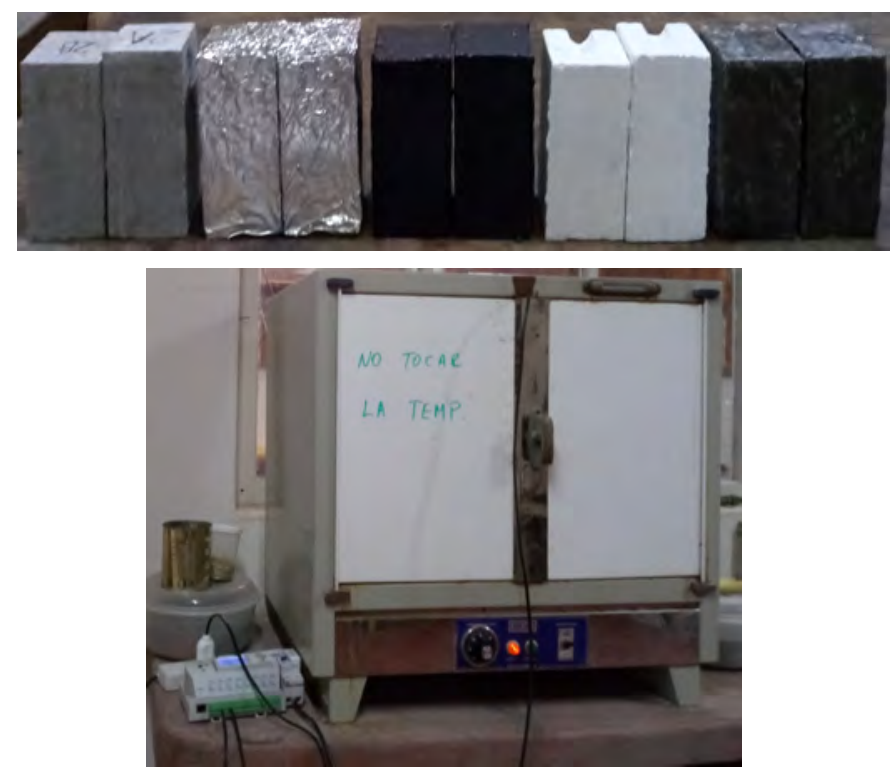

Figura 4.18: Prueba de pinturas de aislación

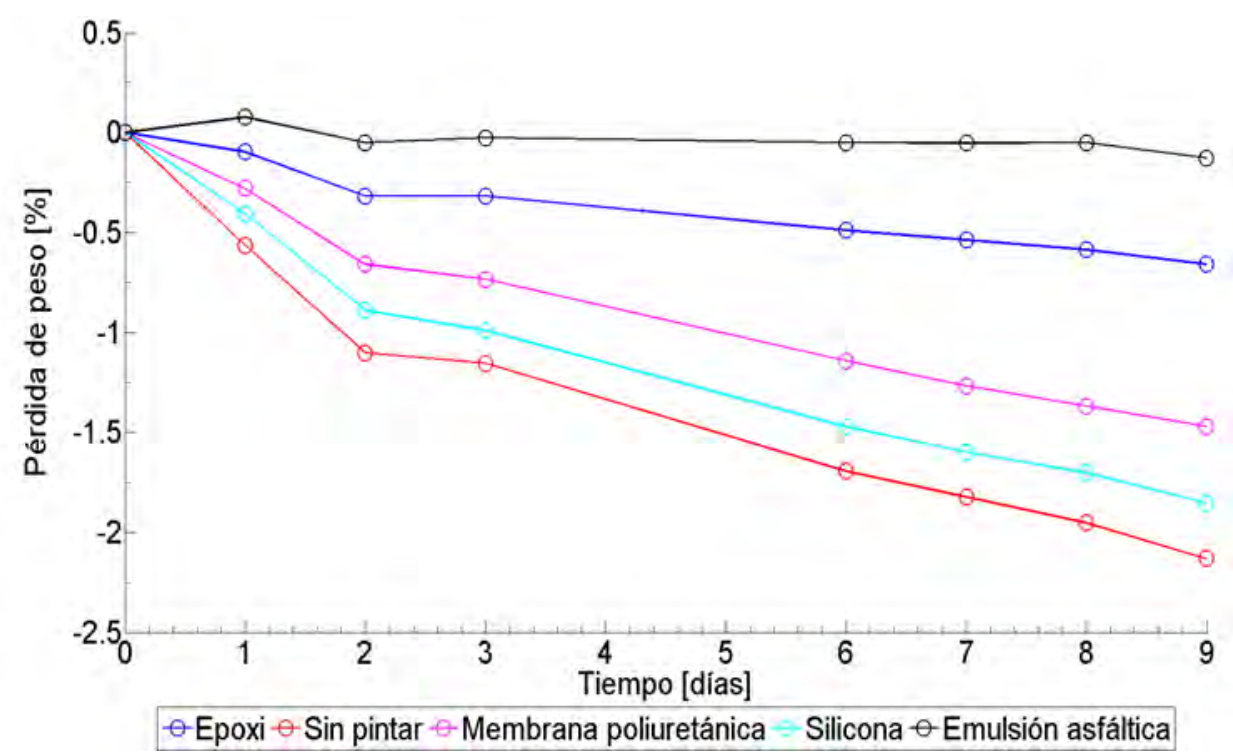

Figura 4.19: Evolución en el tiempo de la pérdida de peso en las probetas con cada tipo de pintura de aislación

hidrostática, provocando una compresión sobre el esqueleto sólido denominada contracción, Mindess et al. (2003). Durante el proceso de hidratación es posible que ocurra un reacomodamiento interno de las partículas de agua, originando la formación de un menisco en algunos poros capilares. Esto puede provocar una contracción sin que ocurra una reducción en el contenido 
de agua, es decir, sin que tenga lugar una pérdida de peso. Por este motivo es necesario realizar el ensayo de contracción libre incluso en las probetas en las que se aplicó el aislante.

Para el ensayo de contracción libre se moldearon doce probetas de 7 x 7 x $30 \mathrm{~cm}$ con pernos en los extremos según lo establecido por la norma IRAM1524 (1982), compactadas manualmente y curadas en cámara seca (HR:60 \%, $\left.\mathrm{T}: 20^{\circ} \mathrm{C}\right)$. Las mediciones de contracción libre se realizaron utilizando un comparador vertical con reloj digital, con una precisión de $1 \times 10^{-3} \mathrm{~mm}$. En la Figura 4.20 se presentan el equipo utilizado y las probetas ensayadas, con las distintas condiciones de aislación.

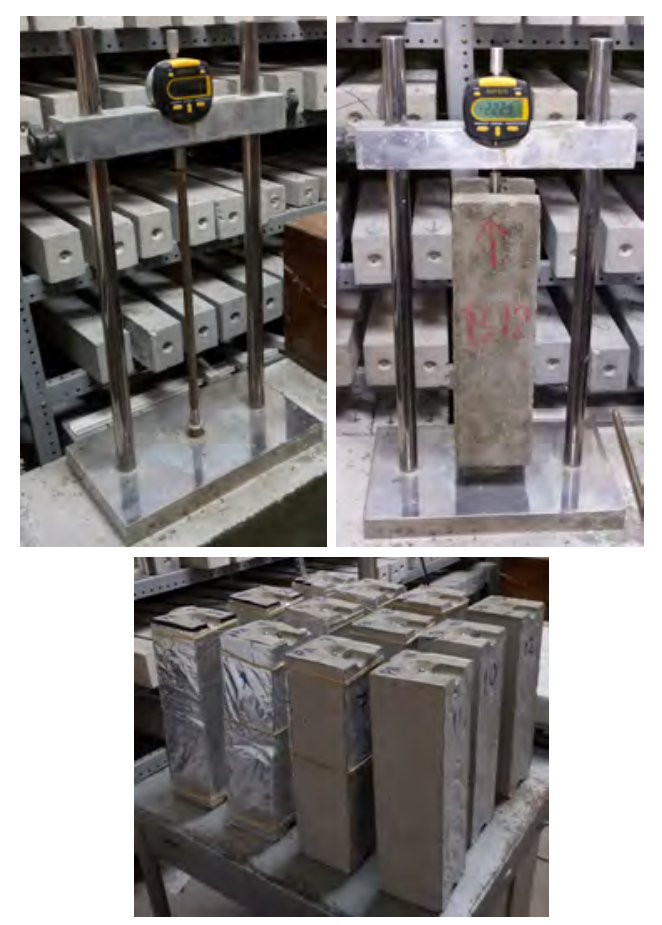

Figura 4.20: Ensayo de contracción libre en probetas prismáticas de 7 x 7 x $30 \mathrm{~cm}$

El ensayo de contracción libre permite determinar los cambios en la longitud de una probeta o testigo de hormigón o mortero sin que se produzca la acción de fuerzas externas o cambios de temperatura, evaluando el potencial de expansión o contracción volumétrica de la probeta del hormigón o mortero estudiado, ASTM (2013a).

Las mediciones fueron llevadas a cabo durante 24 días y también se determinó la pérdida de peso de cada una de las probetas. 


\section{Resultados}

En la Figura 4.21 se observan los valores de contracción promedio para cada una de las condiciones de aislación

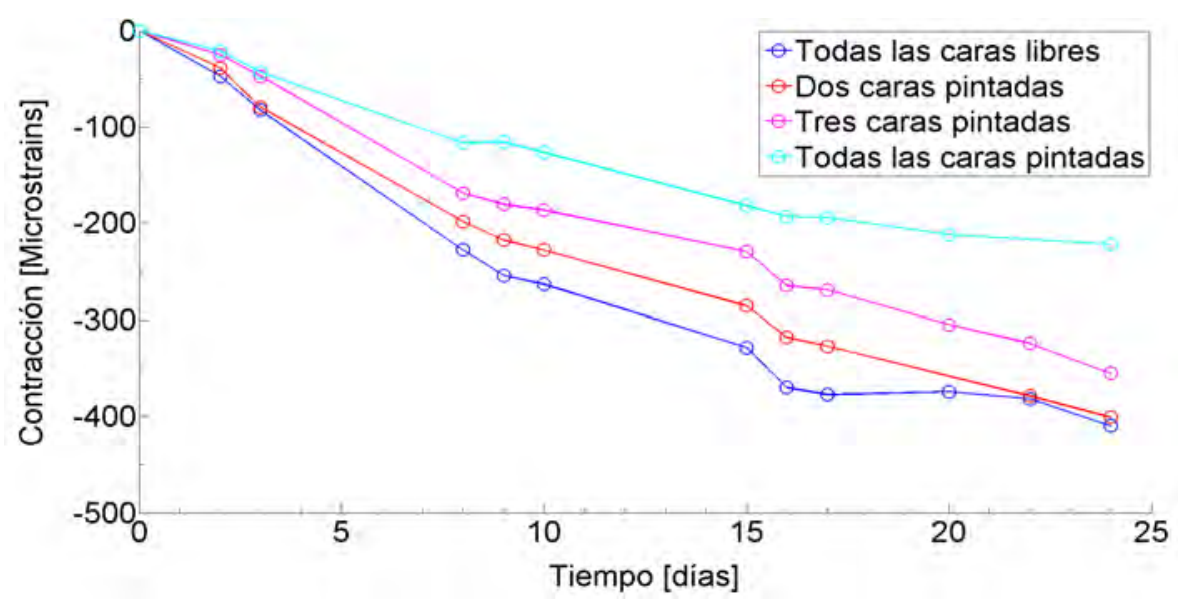

Figura 4.21: Evolución contracción libre en el tiempo para cada condición de aislación

Para el cálculo de las temperaturas equivalentes deben considerarse las relaciones $A / V$ para las distintas condiciones de aislación indicadas en la Tabla 4.11

\begin{tabular}{|c|c|}
\hline Cantidad de caras pintadas & $\mathrm{A} / \mathrm{V}$ \\
\cline { 2 - 2 } & {$[1 / \mathrm{mm}]$} \\
\hline 6 & 0 \\
\hline 2 & 0.029 \\
\hline 1 & 0.014 \\
\hline 0 & 0.057 \\
\hline
\end{tabular}

Tabla 4.11: Relaciones A/V para probetas de $7 \times 7 \times 30 \mathrm{~cm}$ bajo distintas condiciones de aislación

El anillo ensayado solo presenta una cara aislada, su base, por lo que su relación $A / V$ será $0.0361 / \mathrm{mm}$.

Para obtener las temperaturas equivalentes se dividen las deformaciones por contracción libre registradas en cada una de las probetas a distintas edades por el coeficiente de expansión térmica, el cual adopta el valor $1 x 10^{-5} 1 /{ }^{\circ} \mathrm{C}$ en el hormigón, obteniéndose así los valores presentados en la Figura 4.22 .

A partir de ello se obtienen curvas para distintas edades, en las que se vinculan las relaciones $A / V$ con las temperaturas equivalentes, como se observa en la Figura 4.23. 


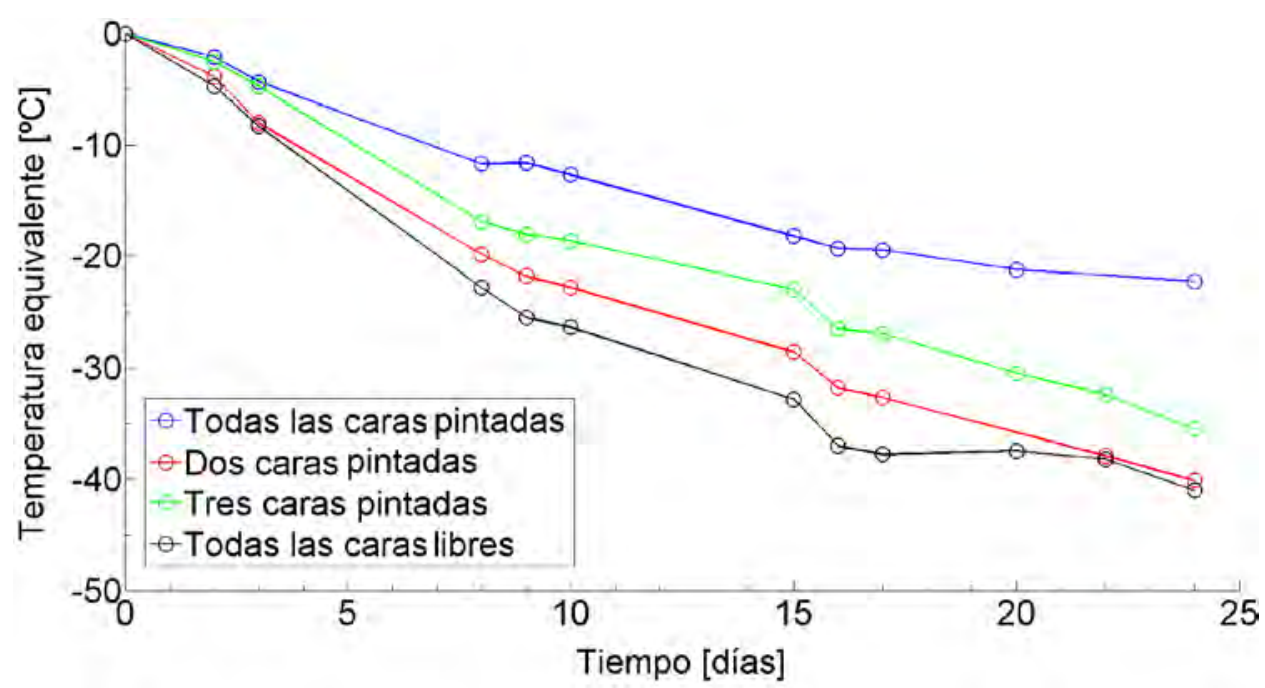

Figura 4.22: Temperaturas equivalentes obtenidas a partir del ensayo de contracción libre

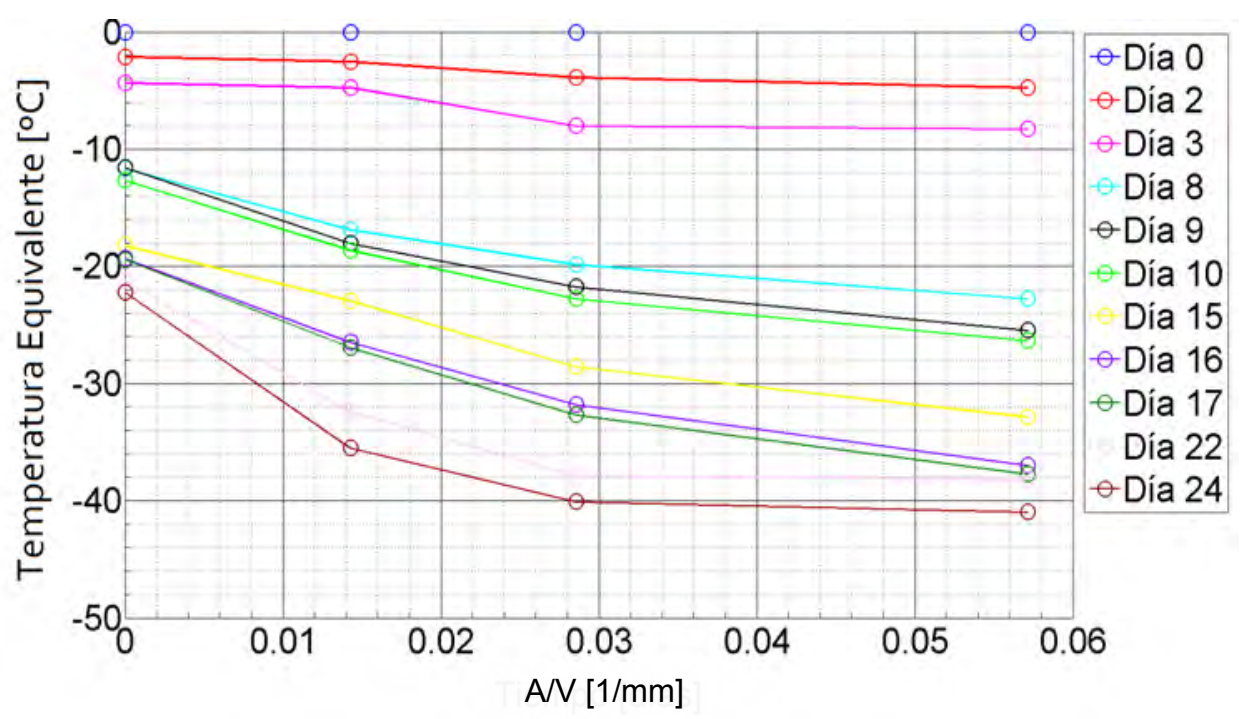

Figura 4.23: Relaciones $A / V$ para cada una de las edades analizadas

Finalmente de la Figura 4.23 se obtienen los valores de temperaturas equivalentes para la relación $A / V$ correspondiente al anillo de hormigón. Con esos puntos se determina el ajuste para calcular la temperatura equivalente en el anillo de hormigón a lo largo del tiempo, como se observa en la Figura 4.24. A continuación se indica la curva de ajuste obtenida para el hormigón estudiado:

$$
\Delta T=0,0517 * t^{2}-2,904 * t+0,308
$$

Ing. M. Paula Zappitelli 
donde $t$ se mide en días

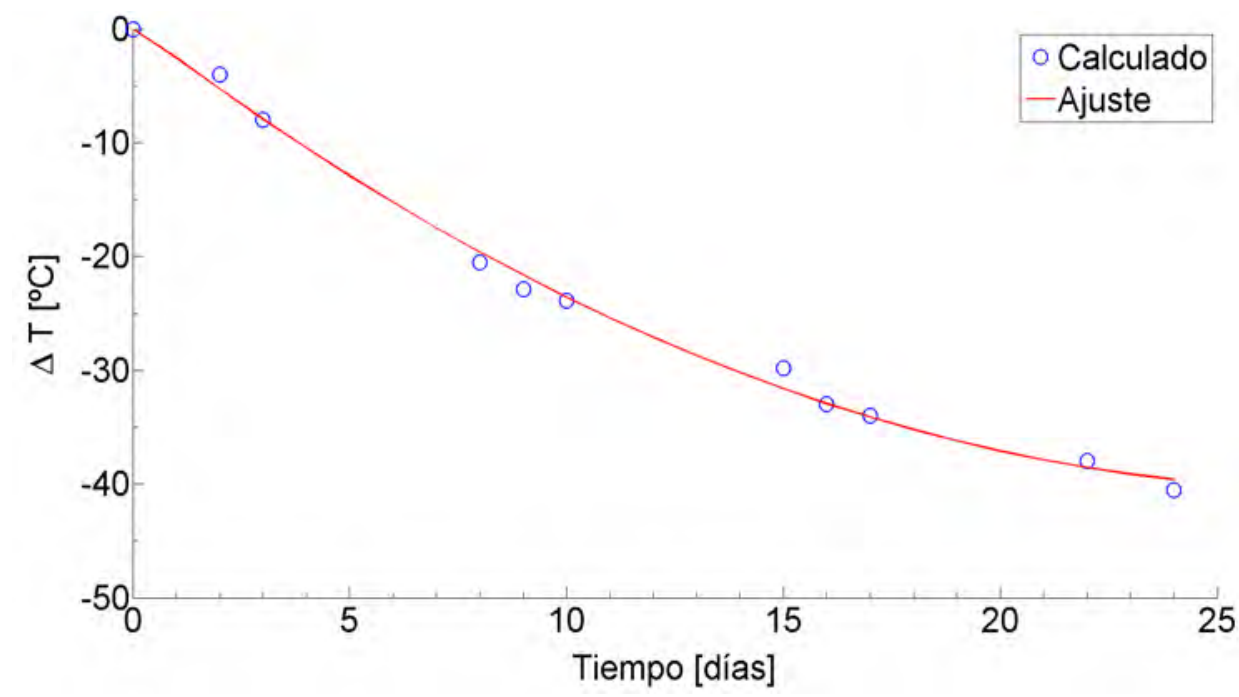

Figura 4.24: Curva de ajuste para el cálculo de las temperaturas equivalentes en el anillo de hormigón

\subsubsection{Determinación de la energía de fractura en Modo I}

Se moldearon tres vigas de $7 \times 10.5$ x $43 \mathrm{~cm}$ según lo establecido por la norma IRAM-1524 (1982), compactadas con vibración externa a través de mesa vibradora y curadas en cámara seca $\left(\mathrm{HR}: 60 \%\right.$, T:20 $\left.{ }^{\circ} \mathrm{C}\right)$. Para la determinación de la energía de fractura se utilizó una máquina Instron (capacidad $150 \mathrm{KN}$ ) con control de desplazamiento, y transductores de desplazamiento lineal (LVDT) y tipo clip aplicados en la probeta.

Se puede definir la energía específica de fractura como la cantidad de energía necesaria para generar un área unitaria fisurada. El área fisurada se define como el área proyectada sobre un plano paralelo a la dirección principal de la fisura.

El ensayo consiste en determinar el área bajo la curva carga-deflexión completa obtenida a partir del ensayo de una viga entallada sometida a flexión en tres puntos. Se realizó una compensación de pesos para eliminar el efecto de volcamiento por peso propio de cada mitad de la probeta. Para ello se fijó a ambos lados de la muestra un marco de acero dotado de un contrapeso, cuya masa y separación del apoyo compensaron el momento producido por la mitad de la viga.

La energía de fractura se calcula como el área bajo la curva cargadeflexión dividida por el área del ligamento sobre la entalla, RILEM (1985).

Las probetas prismáticas se entallaron en el centro, utilizando una cierra de disco con injertos de diamante y de corte húmedo, a una profundidad 5.25 
$\pm 0.5 \mathrm{~mm}$. La luz libre entre apoyos es de $40 \mathrm{~cm}$.

La probeta ensayada se encuentra simplemente apoyada, en cada uno de sus extremos sobre un plato de acero, el cual se apoya sobre un cilindro de acero. La carga se aplica en el medio de la probeta utilizando un cilindro y un plato de acero. El cilindro debe tener la capacidad de deslizarse con mínima resistencia.

En el ensayo se midió la apertura de la entalla (CMOD) y la deflexión de la fibra inferior de la muestra o flecha $(\delta)$. El control adoptado fue la velocidad del desplazamiento del plato de la máquina $(0,1 \mathrm{~mm} / \mathrm{min})$ y el fin de la prueba se configuró para un desplazamiento final de $4 \mathrm{~mm}$ o se interrumpió en el momento que la carga $(\mathrm{P})$ fuese igual a cero.

En la Figura 4.25 se muestra la probeta y el montaje del ensayo, y en la Figura 4.26 el esquema para la compensación de peso.
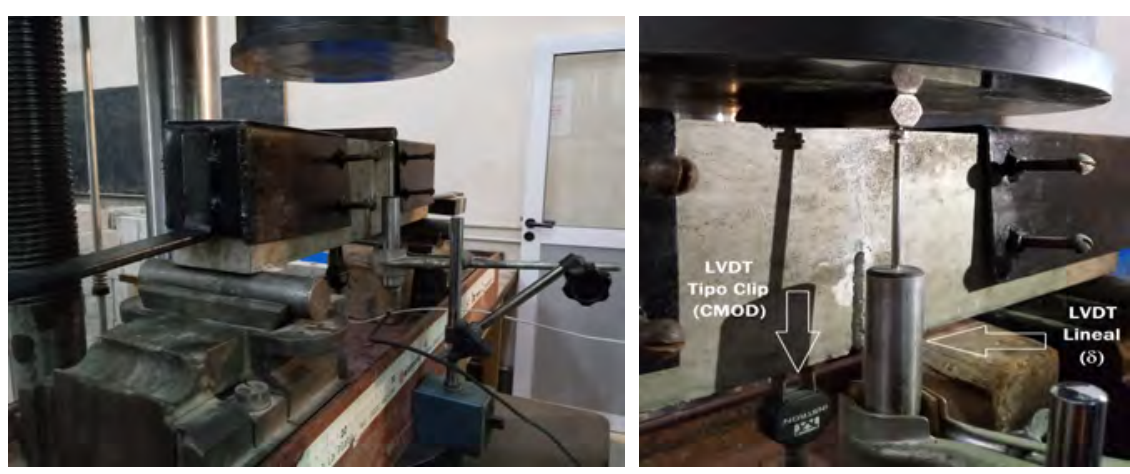

Figura 4.25: Ensayo para la determinación de la energía específica de fractura en modo I

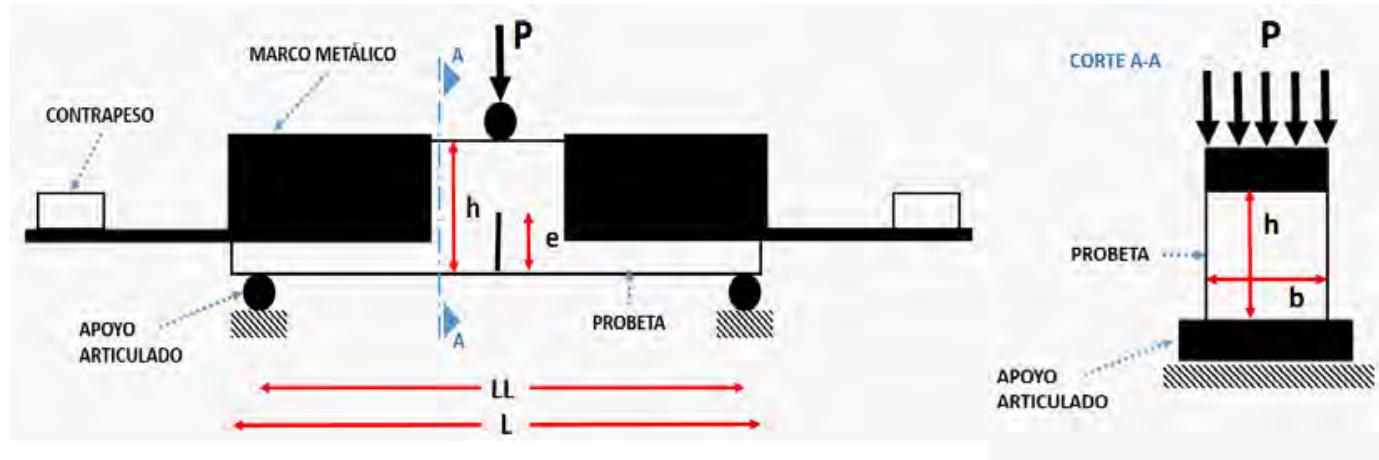

Figura 4.26: Esquema de compensación de pesos para el ensayo de energía de fractura

La energía de fractura se calcula a partir de la expresión 4.10, RILEM

Ing. M. Paula Zappitelli 
(1985):

$$
G_{F}=\frac{W_{0}+m * g * u_{0}}{h_{c} * b}
$$

$W_{0}$ es el trabajo obtenido a partir de la curva carga-deflexión que surge del ensayo, $m$ es la suma entre $m_{1}$ (peso de la viga entre los apoyos) y $m_{2}$ (peso del dispositivo de carga que no está vinculado a la máquina de ensayo, pero sigue el movimiento de la viga hasta la rotura), $g$ es la aceleración de la gravedad, $u_{0}$ es la deflexión en el momentos de la rotura, $h_{c}$ es la profundidad del ligamento sobre la entalla y $b$ es el ancho de la viga.

El ensayo fue llevado a cabo a la edad de 28 días.

\section{Resultados}

En la Figura 4.27 se presenta la curva carga-flecha y en la Figura 4.28 la curva carga-CMOD, para las tres probetas ensayadas.

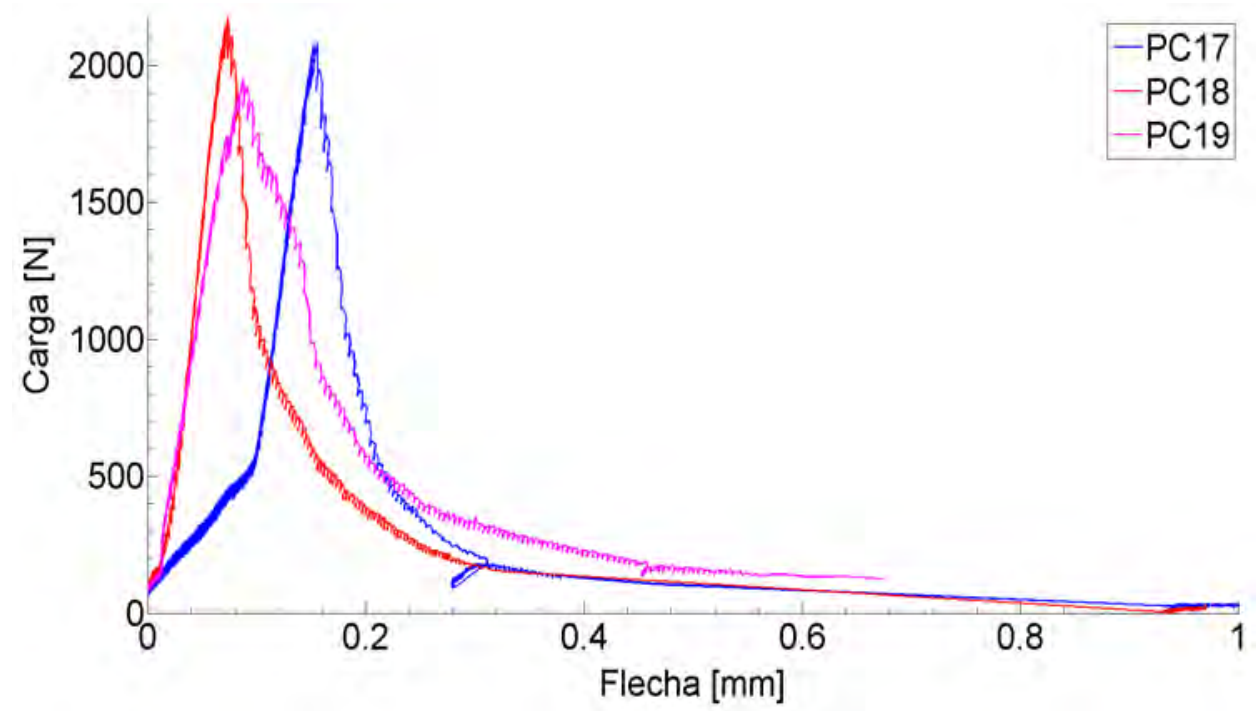

Figura 4.27: Curva carga-flecha para el ensayo de energía de fractura

En la Tabla 4.12 se presenta el valor de la energía de fractura obtenida para cada una de las probetas ensayadas, $G_{f}$, y su valor medio.

\begin{tabular}{|c|c|}
\hline Probeta & $G_{f}\left[\mathrm{Nmm} / \mathrm{mm}^{2}\right]$ \\
\hline PC17 & 0.16 \\
\hline PC18 & 0.12 \\
\hline PC19 & 0.16 \\
\hline Promedio & 0.14 \\
\hline
\end{tabular}

Tabla 4.12: Cálculo de energía de fractura a 28 días 


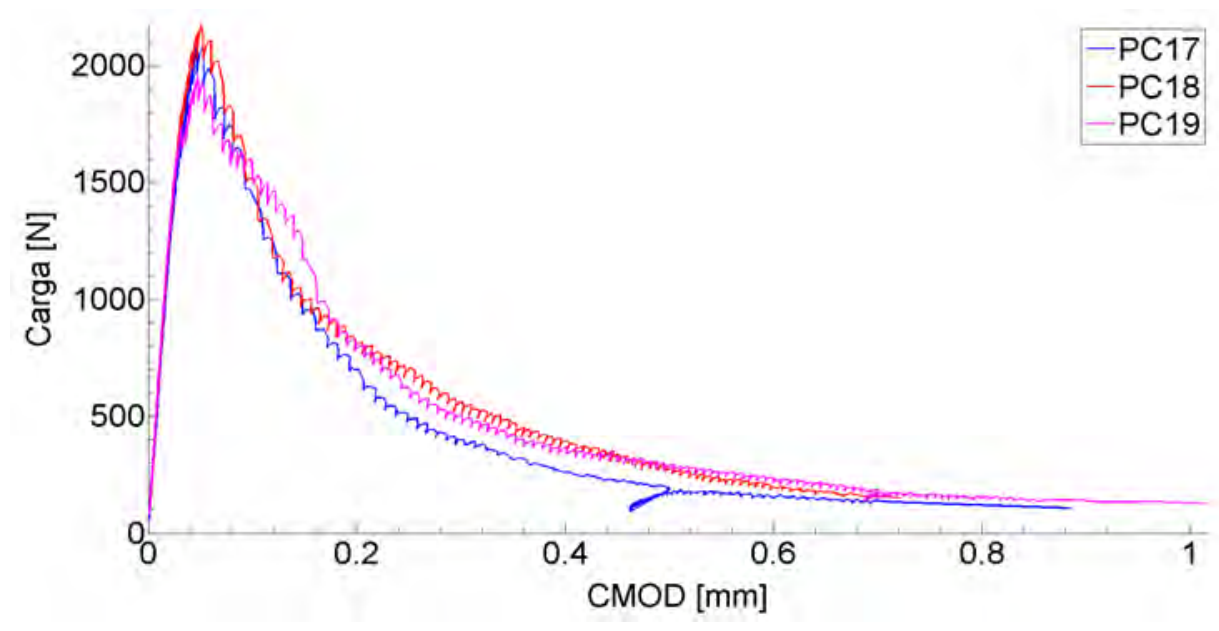

Figura 4.28: Curva carga-CMOD para el ensayo de energía de fractura

Para estimar la evolución en el tiempo de la energía de fractura, ya que solo se contaban con resultados a 28 días, se propuso determinar la relación entre la energía de fractura y la resistencia a compresión y se aplicó este coeficiente a la curva de evolución de la resistencia a compresión, obteniendo así una estimación de la evolución de la energía de fractura en el tiempo. El valor del coeficiente mencionado es $0.00291 / \mathrm{mm}$.

En la Figura 4.29 se observa la estimación de la evolución de $G_{f}$ en el tiempo. La ecuación de ajuste obtenida para dicho gráfico es:

$$
G_{f}(t)=0,0266 \ln (t)+0,0545
$$

donde $t$ es el tiempo medido en días.

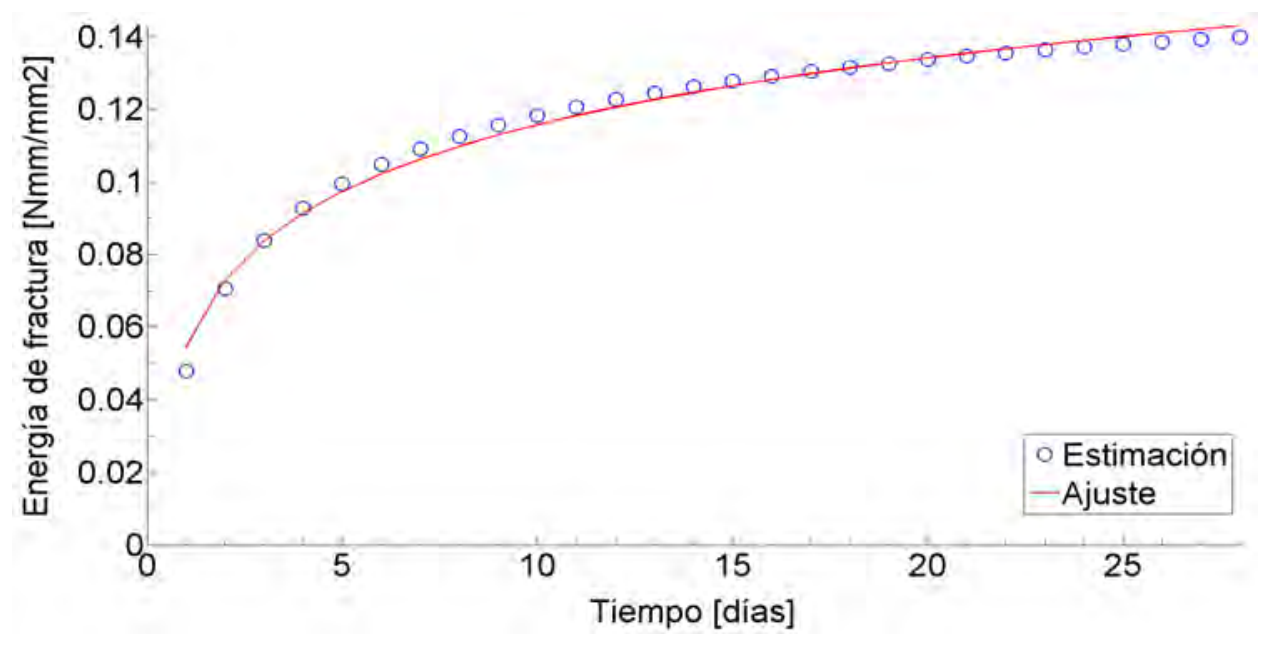

Figura 4.29: Estimación de la evolución de la energía de fractura en el tiempo 


\subsubsection{Ensayo de extensibilidad en tracción}

Se moldearon siete vigas de $7 \times 10.5$ x $43 \mathrm{~cm}$ según lo establecido por la norma IRAM-1524 (1982), compactadas con vibración externa a través de mesa vibradora y curadas en cámara seca (HR:60\%, T:20 ${ }^{\circ} \mathrm{C}$ ). Para el ensayo a carga lenta se utilizó un pórtico con un sistema de palancas. El ensayo a carga rápida se realizó mediante una máquina Instron (capacidad $150 \mathrm{KN}$ ) con control de desplazamiento y se utilizó un transductor de desplazamiento lineal (LVDT).

Se define como extensibilidad a la deformación de las fibras inferiores de una viga para el $95 \%$ de la carga última, Giovambattista et al. (1992).

Para el ensayo a carga lenta en el pórtico de carga se coloca la viga simplemente apoyada sobre cilindros y en la parte inferior de la misma, a la altura de la fibra más traccionada, se fija en un lado un transductor tipo LVDT y en el otro un flexímetro sobre una base de $150 \mathrm{~mm}$, para medir la extensibilidad. La viga se encuentra cargada a los tercios mediante un sistema de palancas en el pórtico de carga mencionado, en cuyo extremo se colocan pesas de acuerdo con los escalones de carga definidos hasta la rotura de la viga, Giovambattista et al. (1992), Giovambattista et al. (1987), Houghton (1976). En este caso se aplicaron diez escalones de carga a lo largo de diez días en una de las vigas y nueve a lo largo de nueve días en la restante. En la Figura 4.30 se observa el pórtico de carga y la viga para el ensayo a carga lenta.

En el caso de los ensayos a carga rápida, se coloca la viga con las mismas condiciones de apoyo y carga descriptas anteriormente (simplemente apoyada y carga a los tercios) en la máquina de ensayos Instron. Se fija un LVDT en la fibra más traccionada para registrar las deformaciones por extensibilidad, pero la carga se aplica en forma rápida. En la Figura 4.31 se muestra el ensayo de extensibilidad a carga rápida.

Se ensayaron dos vigas a carga lenta hasta la rotura durante 10 días, tres vigas a carga rápida el día 1 y tres vigas a carga rápida el día 10.

\section{Resultados}

En la Figura 4.32 se observan los resultados de los ensayos de extensibilidad a carga rápida de 1 día y 10 días y de carga lenta.

Las tensiones de tracción debidas a contracción restringida desarrolladas en el hormigón se reducen por efecto del creep, por lo que resulta de interés cuantificar esta reducción, Biffaut et al. (2011). Se denomina creep a las deformaciones generadas debido a la acción de una carga sostenida en el tiempo. Si bien los materiales metálicos y cerámicos presentan deformaciones por creep, el hormigón se diferencia de ellos en que dichas deformaciones tienen lugar a temperatura ambiente y en que la presencia de humedad es un factor de considerable influencia, Mindess et al. (2003). 


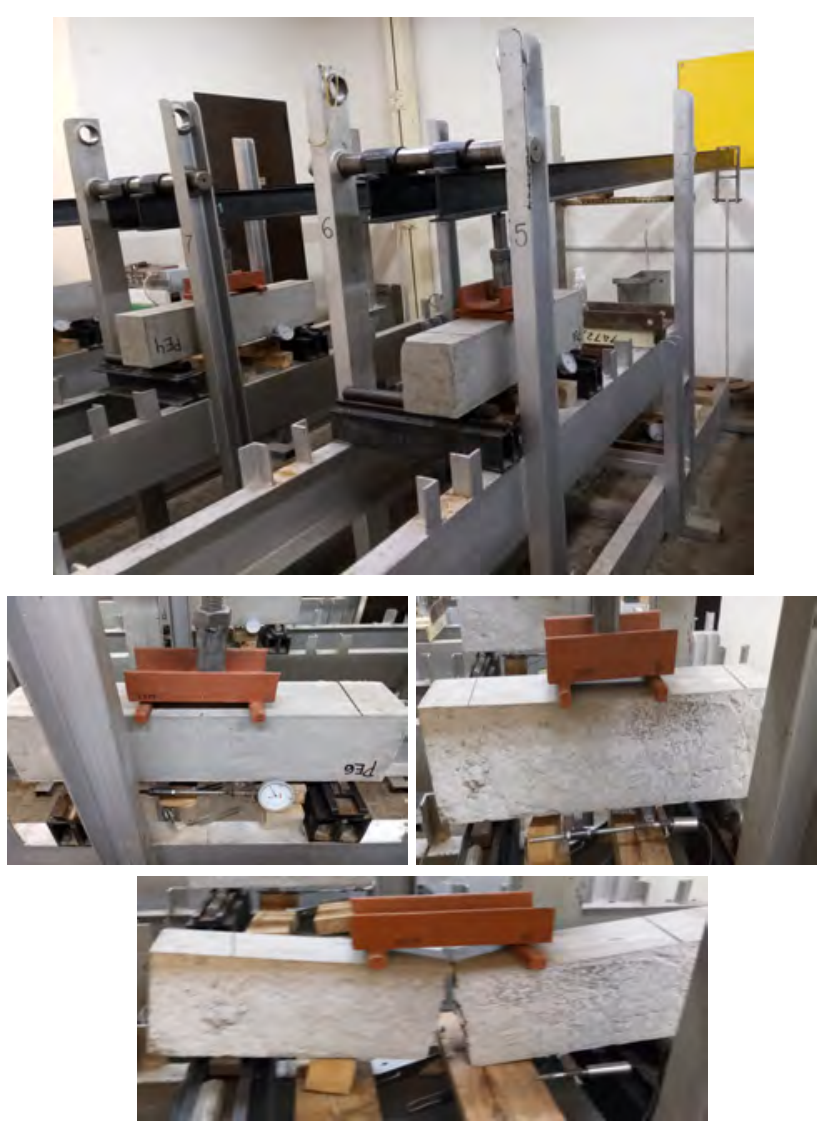

Figura 4.30: Ensayo de extensibilidad a carga lenta
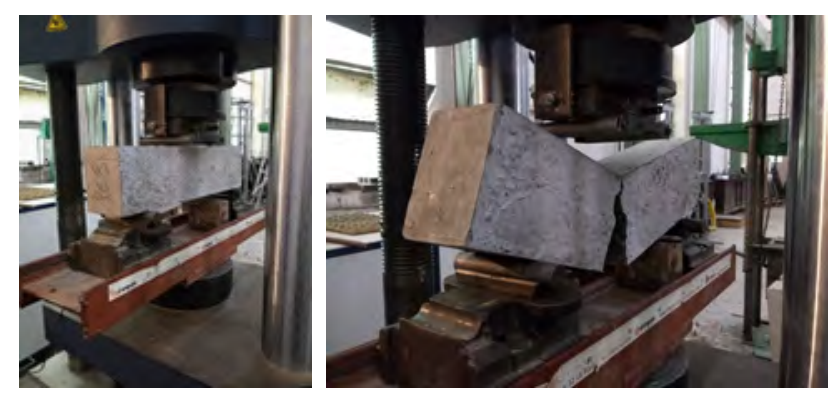

Figura 4.31: Ensayo de extensibilidad a carga rápida

En los modelos numéricos se considera al creep como una deformación diferida simplemente reduciendo el módulo de elasticidad en el tiempo, al módulo reducido se lo denomina módulo efectivo. En la literatura se suele aplicar el valor 0.6 como factor de reducción, Weiss et al. (2000); Moon et al. (2006); Zhou et al. (2014); Dong et al. (2016), pero no se indica por qué motivo se adopta este valor. Por esta razón se propuso realizar un ensayo de 


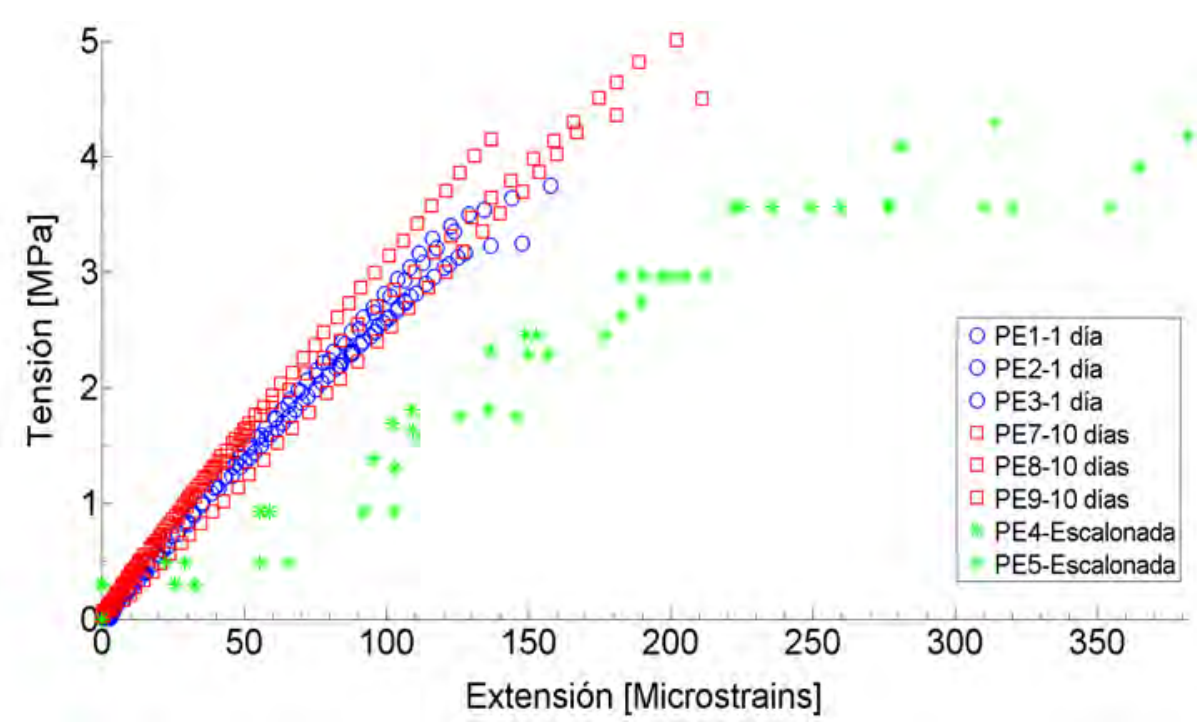

Figura 4.32: Resultados de los ensayos de extensibilidad a carga rápida y lenta

extensibilidad a carga lenta, por el cual tienen lugar deformaciones en las probetas debido a cargas sostenidas en el tiempo, y el mismo ensayo a carga rápida, y correlacionar las deformaciones finales obtenidas al momento de la rotura. Para la determinación del estado de cargas se simuló numéricamente el ensayo de contracción restringida en un anillo con las propiedades mecánicas del hormigón caracterizado en este capítulo, obteniendo el estado tensional del mismo antes de que se produzca la fisuración. Luego se determinó la carga correspondiente a ese estado tensional y ésta se utilizó en el ensayo de extensibilidad.

De esta forma se busca poder determinar un factor que permita estimar el efecto provocado por el creep en el hormigón estudiado a la edad de finalización del ensayo, y mediante un ajuste logarítmico obtener la evolución en el tiempo del mencionado factor.

En este caso, la edad de rotura de las probetas ensayadas a carga lenta fue 10 días, y la deformación promedio alcanzada en tracción a esa edad fue $264 x 10^{-6}$. Por este motivo se realizó el ensayo de carga rápida a esas edad y se obtuvo una deformación final promedio de $182 \times 10^{-6}$, la relación entre ambas deformaciones es igual a 0.69. Aplicando este factor de reducción al módulo de elasticidad a 10 días, se determina un ajuste logarítmico variable en el tiempo, como se observa en la Figura 4.33. A continuación se presenta la ecuación del ajuste:

$$
E_{\text {sostenido }}(t)=-841 \ln (t)+19956
$$

donde $t$ es el tiempo medido en días. 


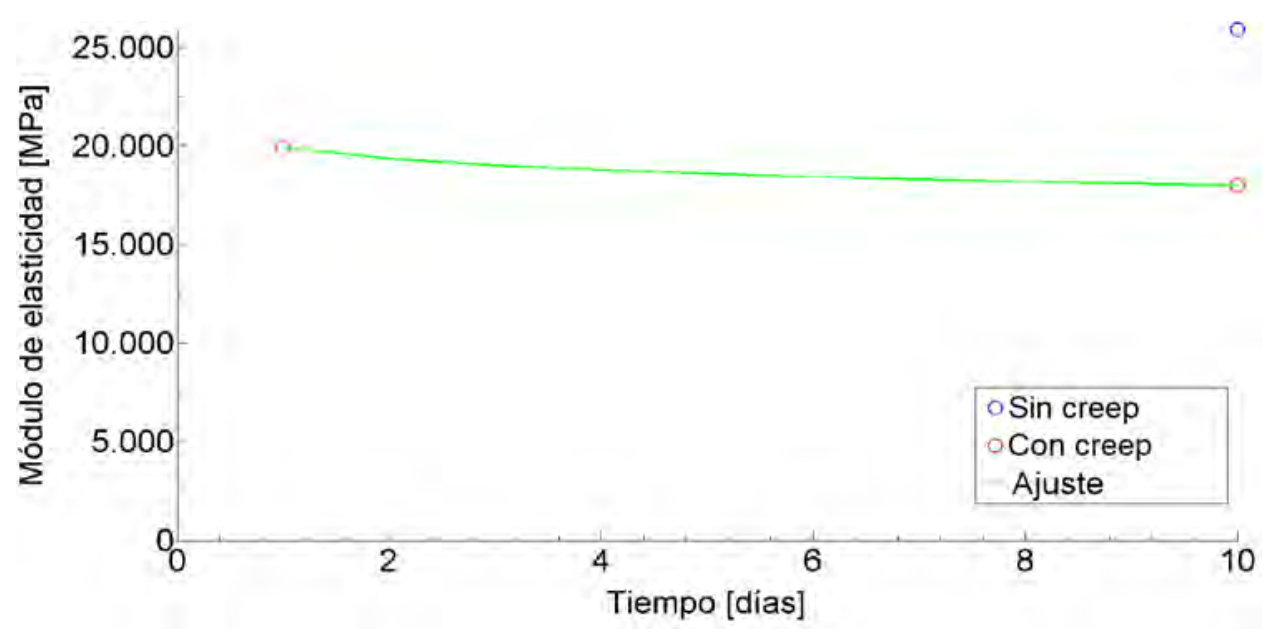

Figura 4.33: Ajuste del módulo de elasticidad considerando el efecto del creep

\subsection{Ensayos de validación}

\subsubsection{Ensayo de contracción restringida en anillos sin enta- llas}

Para la validación del algoritmo desarrollado se llevó a cabo el ensayo de contracción restringida en anillos, descripto en la etapa preliminar.

Se moldearon seis anillos utilizando el hormigón seleccionado en la etapa preliminar, con un contenido de cemento de $600 \mathrm{Kg} / \mathrm{m}^{3}$, de acuerdo con la norma ASTM (2004a). Los anillos fueron curados en cámara seca (HR:60 \%, $\left.\mathrm{T}: 20^{\circ} \mathrm{C}\right)$.

Con el objetivo de determinar el efecto de la edad de desmolde de los anillos en la medición de las deformaciones, a partir de transcurridas $2 \mathrm{hs}$ del hormigonado se comenzaron a aflojar los tornillos interiores y exteriores de cada uno de los anillos. Luego de trascurridas 8 horas se desmoldaron los anillos 1,2 y 5 y a las 24 horas los anillos 3,4 y 6 . A partir de los registros realizados se determinó que el momento en que se aflojan los tornillos y la edad de desmolde no tienen ningún efecto en el comportamiento inicial del hormigón, ya que todos los anillos presentan un cambio de pendiente en las lecturas de deformaciones entre los 0.25 y 0.4 días ( 6 y 9.6 horas), como puede observarse en la Figura 4.34, tanto en los desmoldados a las 8 horas $(1,2$ y 5$)$ como los desmoldados a las 24 horas $(3,4$ y 6$)$. Luego ese comportamiento se estabiliza y se observan a partir del día 1 oscilaciones propias de las lecturas.

\section{Resultados}

Con el objetivo de determinar la edad de fisuración del hormigón utilizado, y adicionalmente al registro continuo de deformaciones en el anillo de 


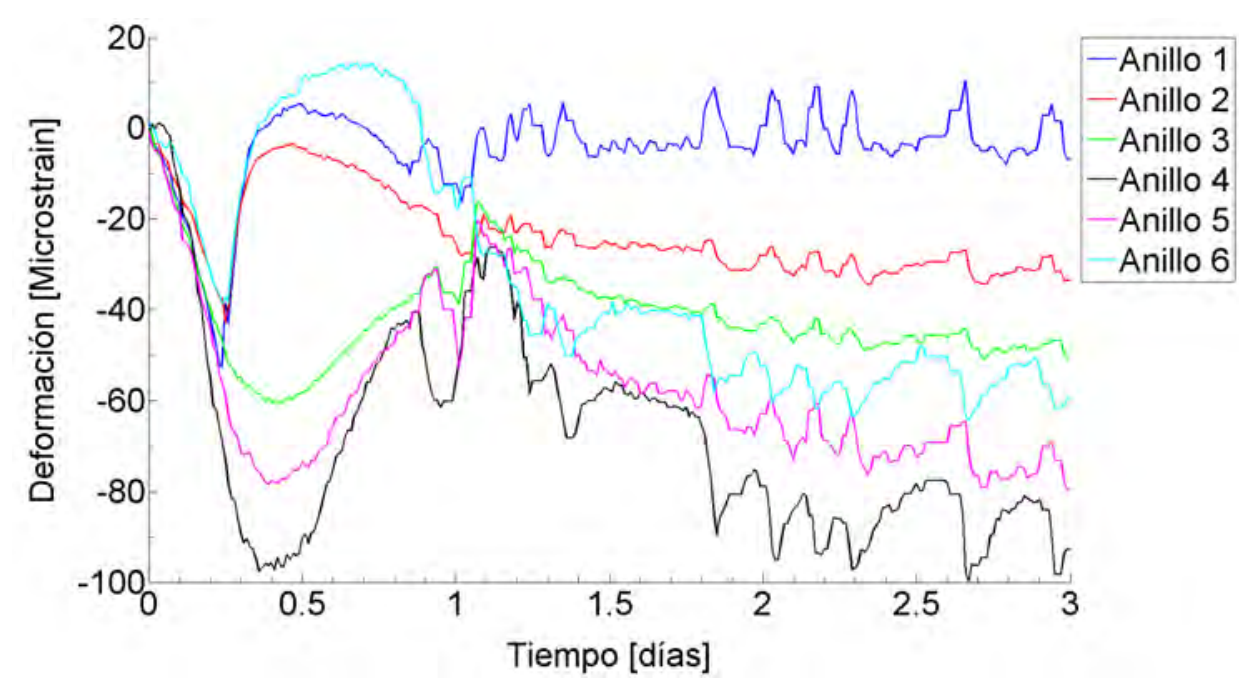

Figura 4.34: Deformaciones medidas en los anillos de contracción restringida durante los primeros días

acero, se llevó a cabo la inspección visual de los anillos de hormigón mediante la utilización de una lupa, una lámpara y el registro de material fotográfico, durante el tiempo de duración del ensayo.

En la Tabla 4.13 se indica lo registrado a partir de la inspección visual.

\begin{tabular}{|c|c|c|c|c|c|c|}
\hline \multirow{2}{*}{$\begin{array}{c}\text { Edad } \\
\text { [días }]\end{array}$} & \multicolumn{6}{|c|}{ Número de Anillo } \\
\cline { 2 - 7 } & 1 & 2 & 3 & 4 & 5 & 6 \\
\hline 1 & FS & SF & FS & SF & SF & FS \\
\hline 4 & SC & SC & SC & SC & FS & SC \\
\hline 5 & SC & SC & SC & SC & SC & SC \\
\hline 6 & SC & SC & SC & SC & SC & SC \\
\hline 7 & SC & SC & SC & SC & SC & SC \\
\hline 8 & $\mathbf{F}$ & SC & $\mathbf{F}$ & $\mathbf{F}$ & $\mathbf{F}$ & $\mathbf{F}$ \\
\hline 11 & $\mathbf{F}$ & $\mathbf{F}$ & $\mathbf{F}$ & SC & $\mathbf{F}$ & SC \\
\hline 12 & SC & SC & SC & SC & SC & SC \\
\hline
\end{tabular}

Tabla 4.13: Registro visual del ensayo de contracción restringida en anillos sin entallas. FS (fisura superficial), SC(sin cambios), F(fisura)

En las Figura 4.35,4.36, 4.37, 4.38, 4.39 y 4.40 se observan los registros continuos de deformaciones para cada uno de los anillos, y en la Figura 4.41 se muestran algunos de los anillos fisurados.

El registro visual llevado a cabo durante 12 días indica que en los anillos $1,3,4,5$ y 6 , aparece una fisura entre $\operatorname{los} 7$ y 8 días de iniciado el ensayo y en el anillo 2 entre los 8 y 11 días. A su vez en los anillos 1,3 y 5 se observó una segunda fisura a esta misma edad. 


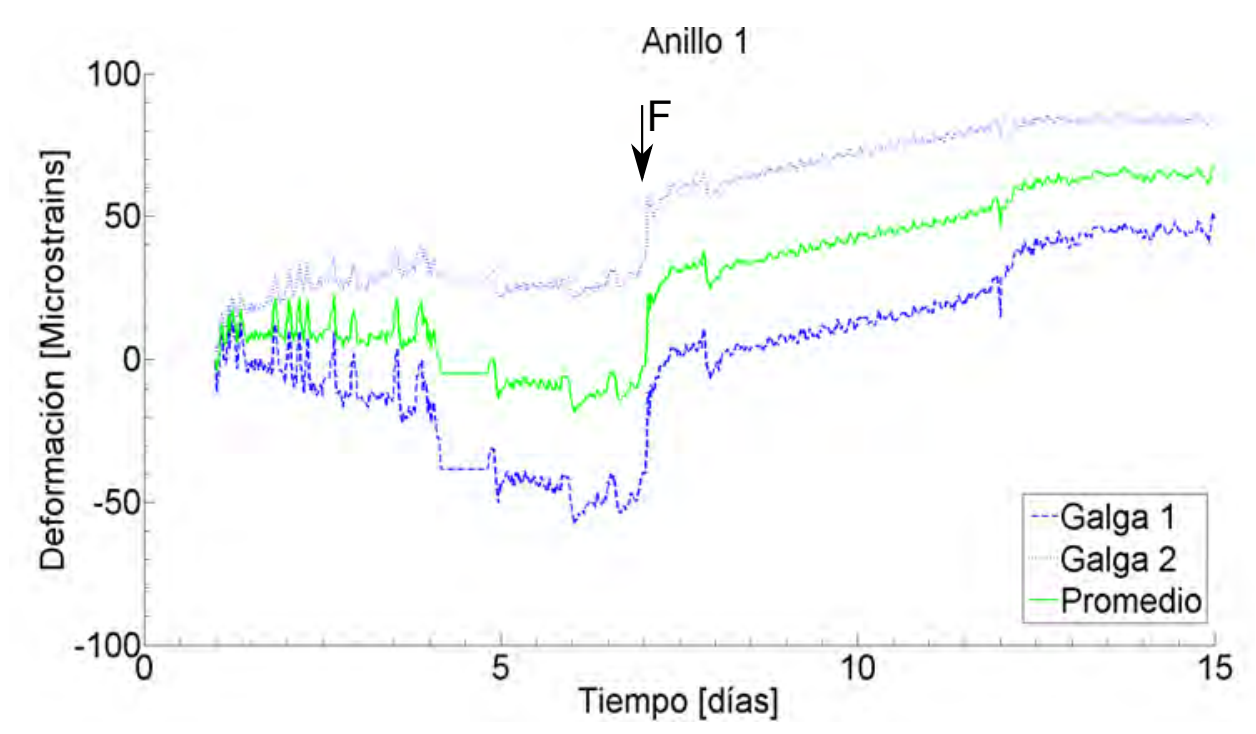

Figura 4.35: Registros de deformaciones para el ensayo de contracción restringida-Anillo 1

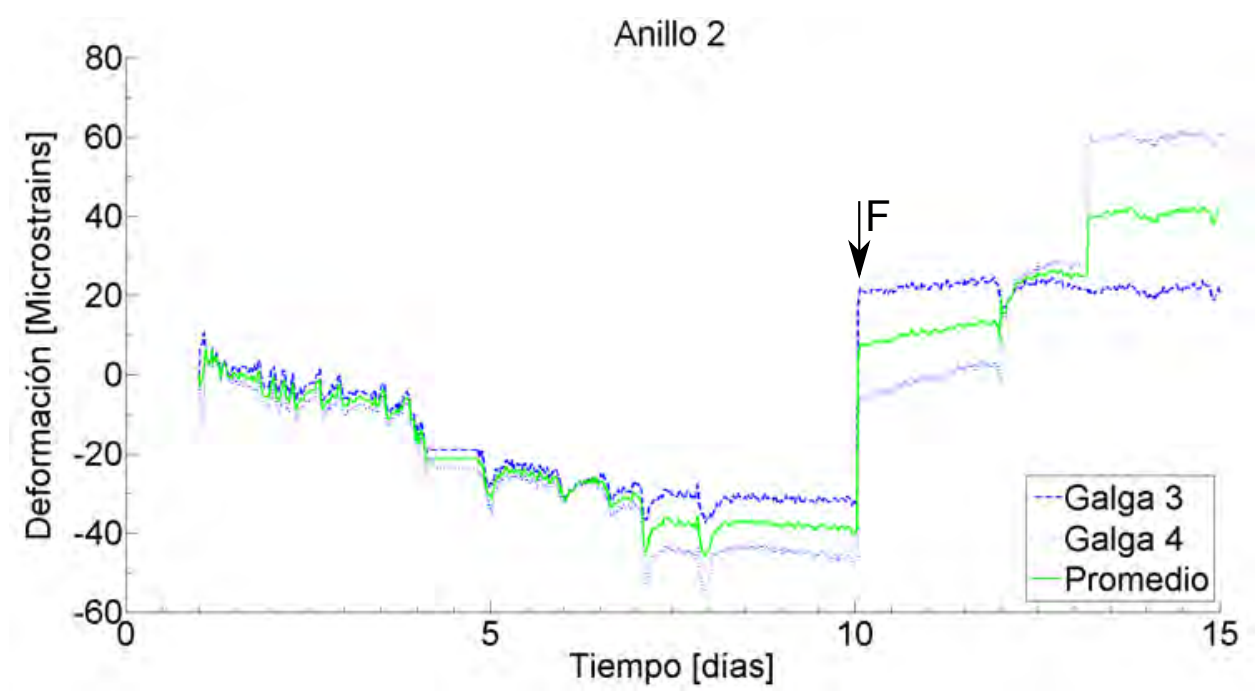

Figura 4.36: Registros de deformaciones para el ensayo de contracción restringida-Anillo 2

Los gráficos correspondientes a los registros continuos se muestran a partir del día 1, momento en que se realiza el desmolde de los anillos, como se observa en los gráficos las fisuras se manifiestan como un salto con cambio de pendiente. Para los anillos 1, 2, 3, 4 y 6, Figuras 4.35, 4.36, 4.37, 4.38 y 4.40 , los saltos resultan pronunciados. En el anillo 5, Figura 4.39, el salto resulta menos evidente, esto puede relacionarse con una posición equidistante de la fisura con respecto a las galgas, sin embargo se observa el cambio de 


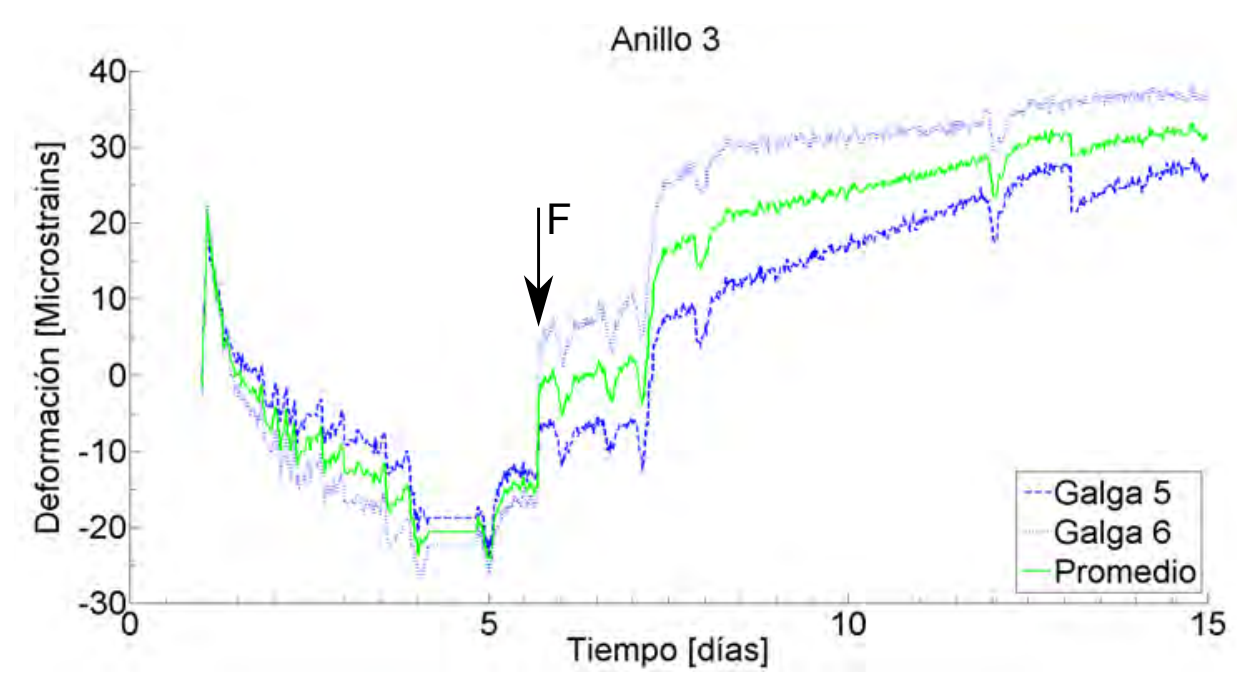

Figura 4.37: Registros de deformaciones para el ensayo de contracción restringida-Anillo 3

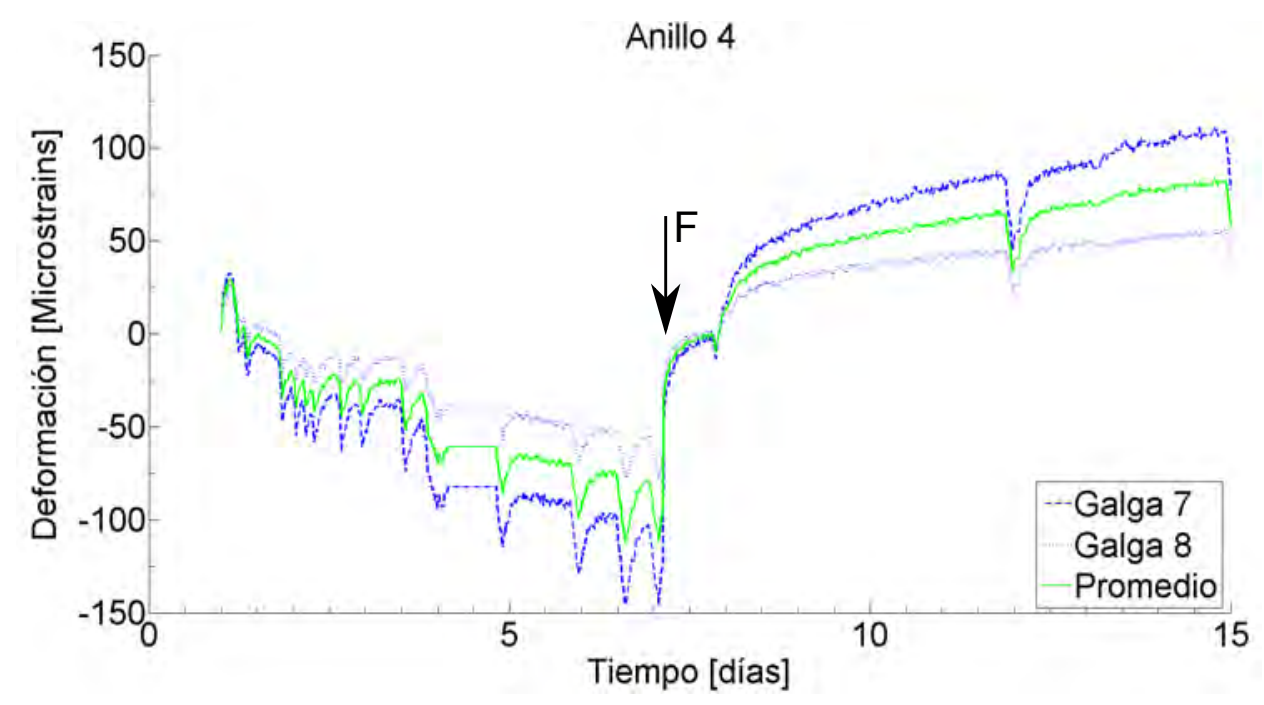

Figura 4.38: Registros de deformaciones para el ensayo de contracción restringida-Anillo 4

pendiente. Otro factor que influye para que el salto no sea pronunciado es la existencia de fricción entre el anillo de acero y el de hormigón, por lo cual el acero sigue tomando carga. En todos los casos el tiempo de aparición de la fisura se encuentra indicado en los gráficos mediante una flecha.

En la Tabla 4.14 se indican los tiempos de aparición resultantes de los registros continuos.

La edad de fisuración obtenida a partir de la observación visual fue de entre 7 y 8 días y el valor promedio obtenido a partir del registro continuo 


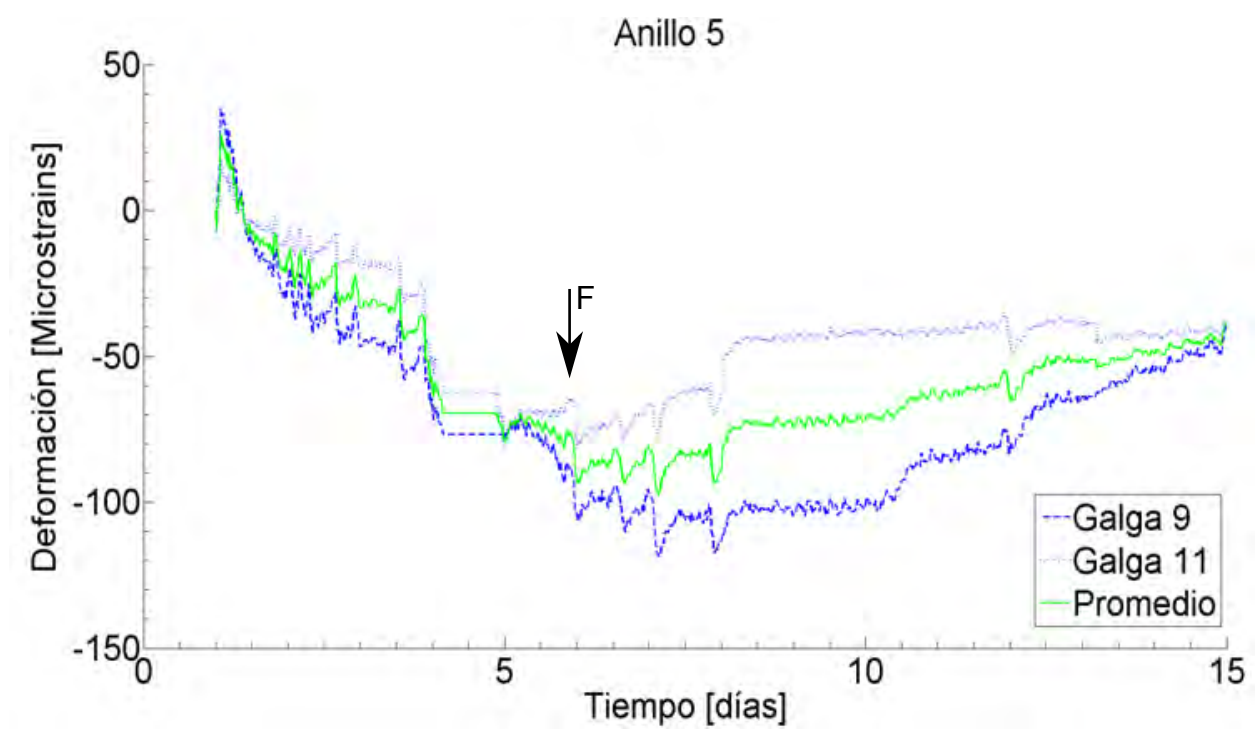

Figura 4.39: Registros de deformaciones para el ensayo de contracción restringida-Anillo 5

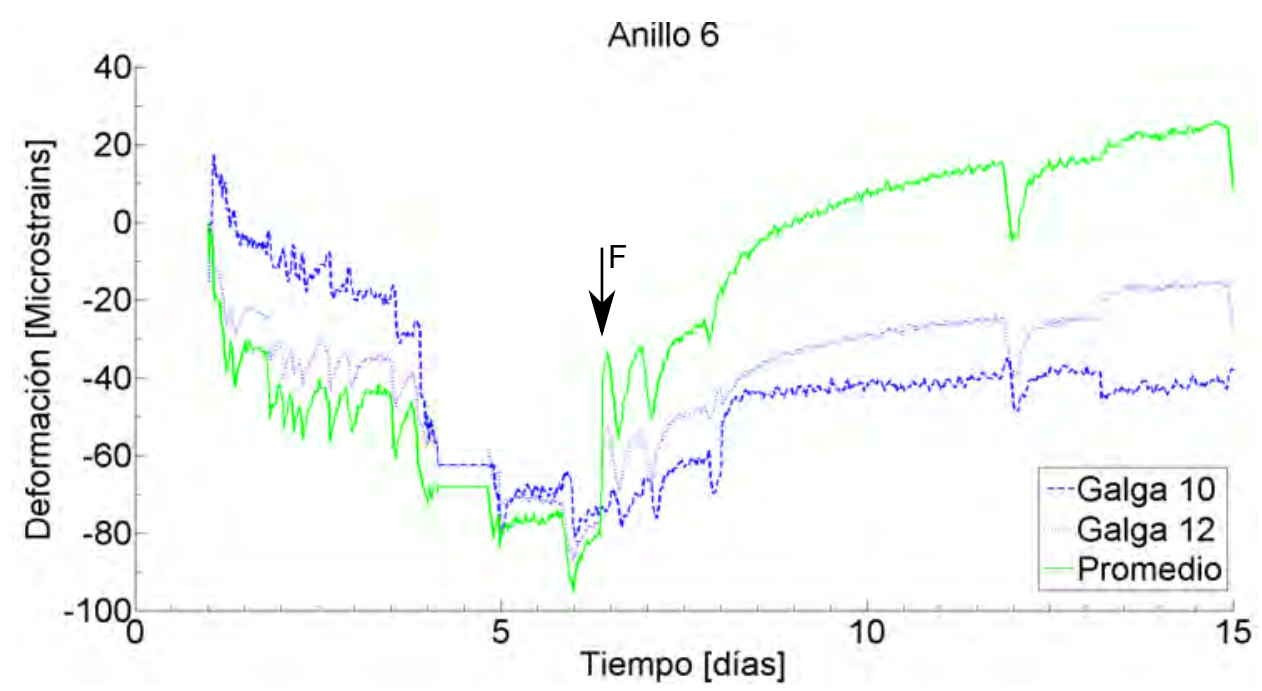

Figura 4.40: Registros de deformaciones para el ensayo de contracción restringida-Anillo 6

fue de 7.05 días. Los resultados de ambas mediciones son similares ya que la diferencia entre los mismos es menor a 1 día. 

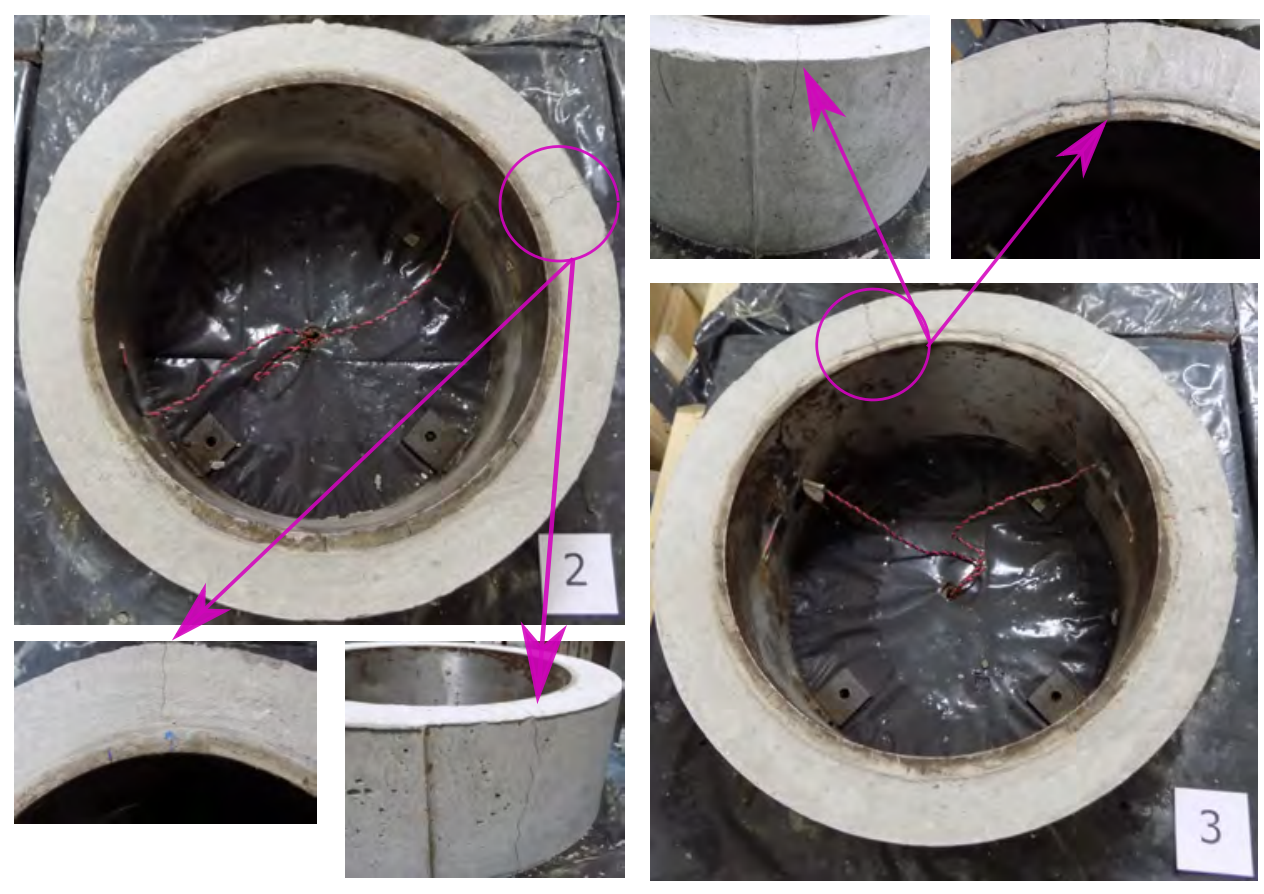

Figura 4.41: Ensayo de contracción restringida en anillos de hormigón sin entalla-Fisuras observadas

\begin{tabular}{|c|c|}
\hline Número de anillo & Edad de fisuración [días] \\
\hline 1 & 7.06 \\
\hline 2 & 10.05 \\
\hline 3 & 5.71 \\
\hline 4 & 7.14 \\
\hline 5 & 5.97 \\
\hline 6 & 6.39 \\
\hline Promedio & 7.05 \\
\hline
\end{tabular}

Tabla 4.14: Tiempos de aparición de fisuras obtenidos a partir de los registros continuos-Anillos sin entalla

\subsubsection{Ensayo de contracción restringida en anillos con enta- llas}

Se moldearon cuarto anillos de hormigón utilizando el pastón de 600 $\mathrm{Kg} / \mathrm{m}^{3}$, de acuerdo con la norma ASTM (2004a). Los mismos fueron curados en cámara seca (HR:60\%, T:20 $\mathrm{C}$ ).

Con el objetivo de estudiar la influencia de la presencia de un defecto en la edad de fisuración del anillo, se le practicó una entalla de $1.5 \mathrm{~cm}$ de largo a tres de los anillos moldeados, como se observa en la Figura 4.42, dejando 
uno solo de ellos sin entalla, para utilizarlo como anillo de control. La entalla se practicó utilizando una chapa plana de $1.5 \mathrm{~cm}$ de ancho, colocada en el extremo del anillo al momento del moldeo.

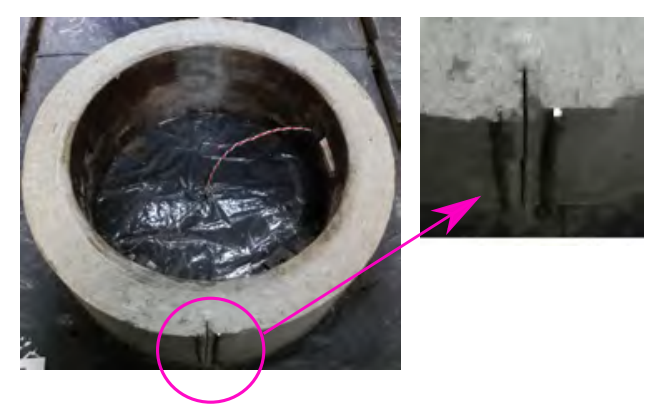

Figura 4.42: Anillos entallados

\section{Resultados}

Al igual que en la sección anterior, se llevó a cabo el registro continuo de las deformaciones en los anillos y se realizó una inspección visual con el objetivo de determinar el momento de aparición de las fisuras.

En las Figuras 4.43, 4.44, 4.45 y 4.46 se observan los registros continuos de deformaciones para cada uno de los anillos, en la Tabla 4.15 se indica lo registrado a partir de la inspección visual, y en la Figura 4.47 las fisuras observadas en algunos de los anillos.

\begin{tabular}{|c|c|c|c|c|}
\hline \multirow{2}{*}{$\begin{array}{c}\text { Edad } \\
\text { [días] }\end{array}$} & \multicolumn{4}{|c|}{ Número de Anillo } \\
\cline { 2 - 5 } & 3 & 4 & 5 & 6 \\
\hline 1 & SC & SC & SC & SC \\
\hline 2 & SC & SC & SC & SC \\
\hline 3 & SC & SC & SC & SC \\
\hline 4 & SC & SC & $\mathbf{F}$ & SC \\
\hline 6 & SC & $\mathbf{F}$ & SC & $\mathbf{F}$ \\
\hline 7 & SC & SC & SC & $\mathbf{F}$ \\
\hline 8 & $\mathbf{F}$ & SC & SC & SC \\
\hline 9 & SC & SC & SC & SC \\
\hline 10 & SC & SC & SC & SC \\
\hline 13 & $\mathbf{F}$ & SC & SC & SC \\
\hline 14 & SC & SC & SC & SC \\
\hline 15 & SC & SC & SC & SC \\
\hline
\end{tabular}

Tabla 4.15: Registro visual del ensayo de contracción restringida en anillos con entallas. FS (fisura superficial), SC(sin cambios), F(fisura) 


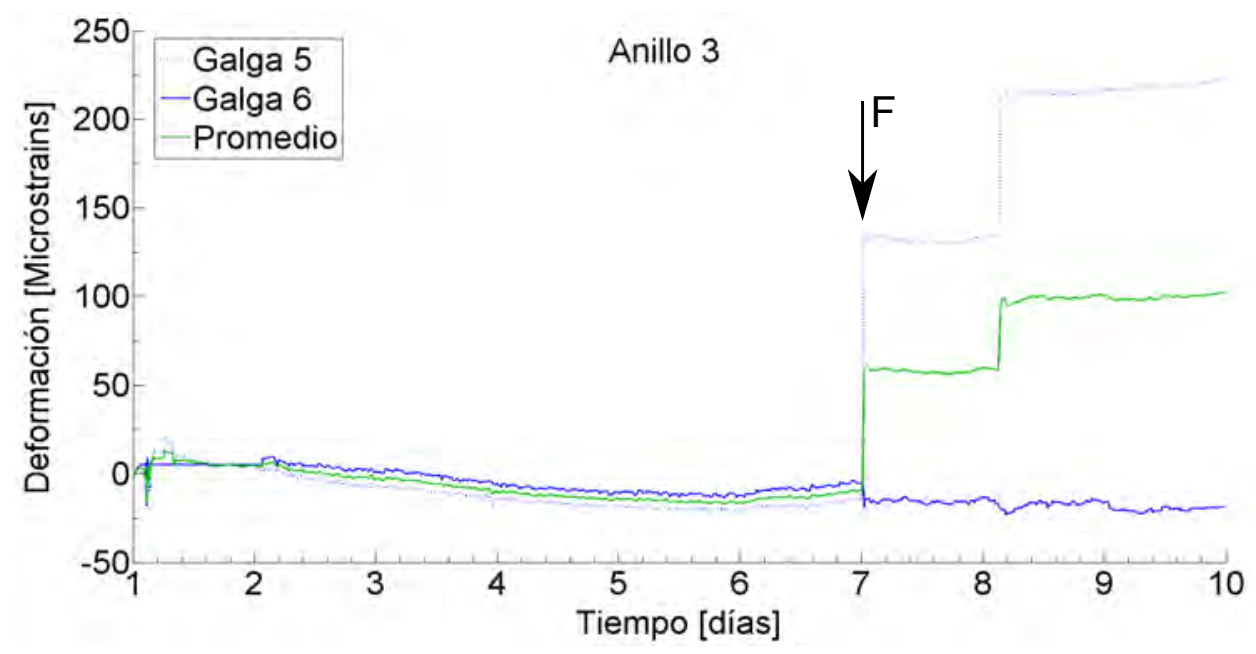

Figura 4.43: Registros de deformaciones para el ensayo de contracción restringida-Anillo 3 sin entalla

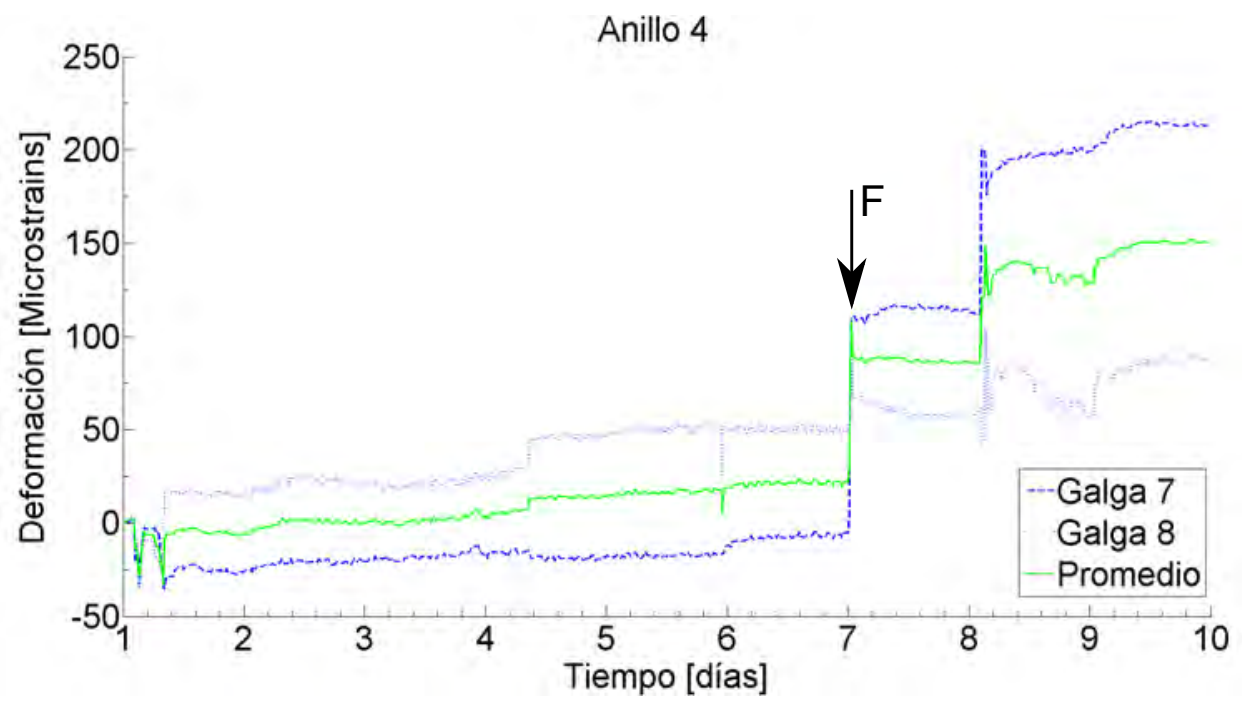

Figura 4.44: Registros de deformaciones para el ensayo de contracción restringida-Anillo 4 entallado

El registro visual indica que los anillos entallados se fisuraron entre los 4 y 7 días de iniciado el ensayo. Por otro lado en el anillo 3, el cual no presentaba entalla, se pudo observar una primera fisura a la edad de 8 días y una segunda fisura a la edad de 13 días.

Si se analizan los registros continuos de deformaciones en los anillos 3,4 y 6 se observan saltos con cambios de pendiente, indicados con una flecha en las Figuras 4.43, 4.44 y 4.46, evidenciando la presencia de una fisura. En cuanto al registro del anillo 5, Figura 4.45, no se observa el salto en el res- 


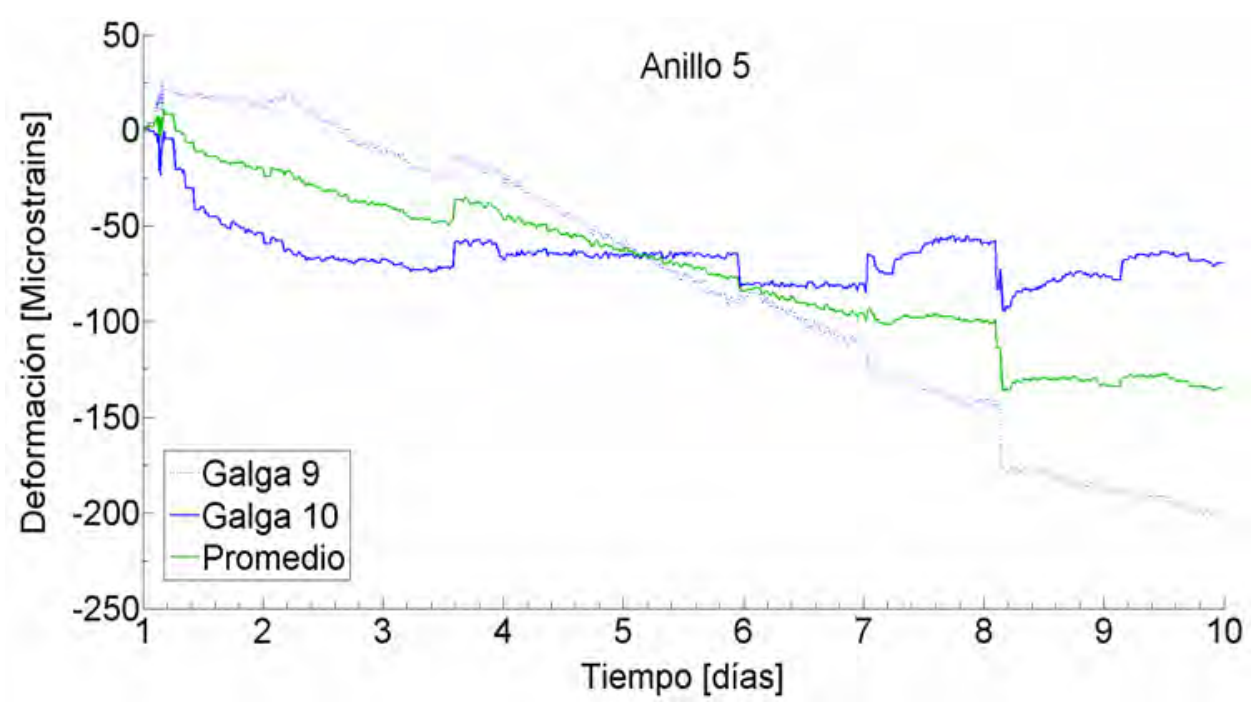

Figura 4.45: Registros de deformaciones para el ensayo de contracción restringida-Anillo 5 entallado

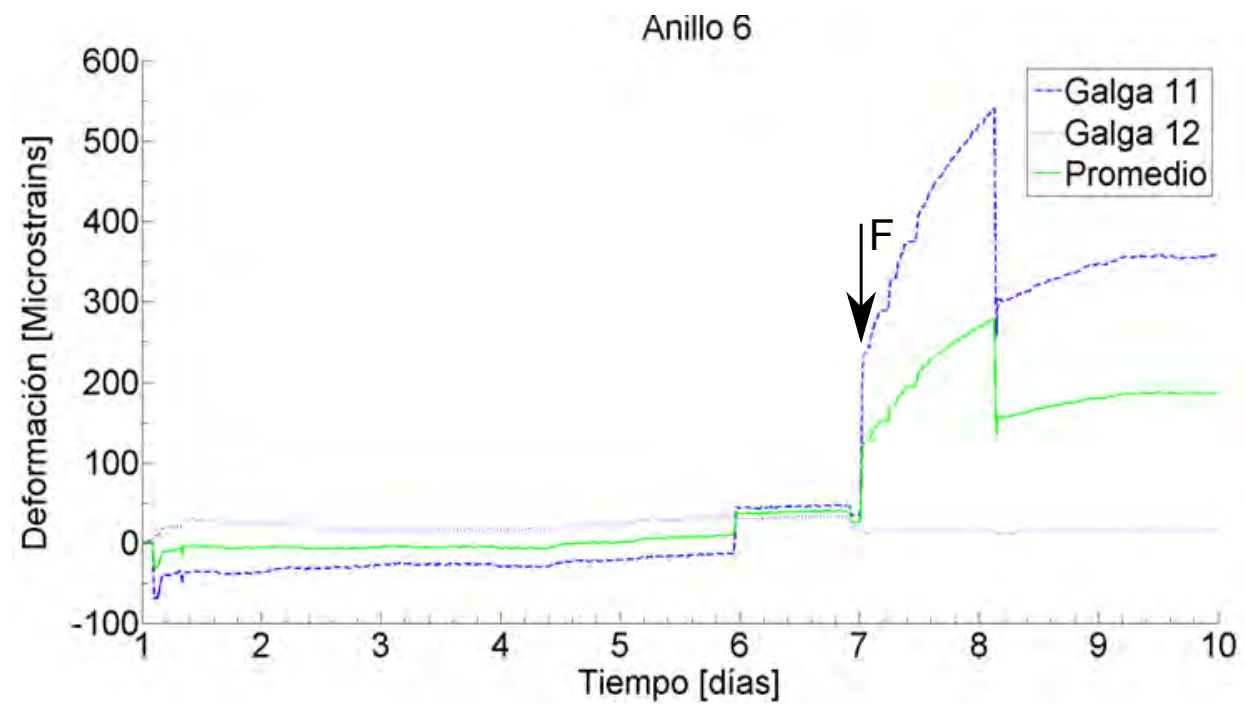

Figura 4.46: Registros de deformaciones para el ensayo de contracción restringida-Anillo 6 entallado

gistro de deformaciones, a pesar de que a partir de la observación visual se verifica la aparición y propagación de la fisura. Esto puede estar vinculado a un error de funcionamiento de las glagas extensométricas colocadas en este anillo.

En la Tabla 4.16 se indican las edades de aparición de fisuras determinadas a partir del registro continuo de deformaciones. Para el anillo 5 no puede extraerse la edad de fisuración de estos registros por lo referido anteriormente. 

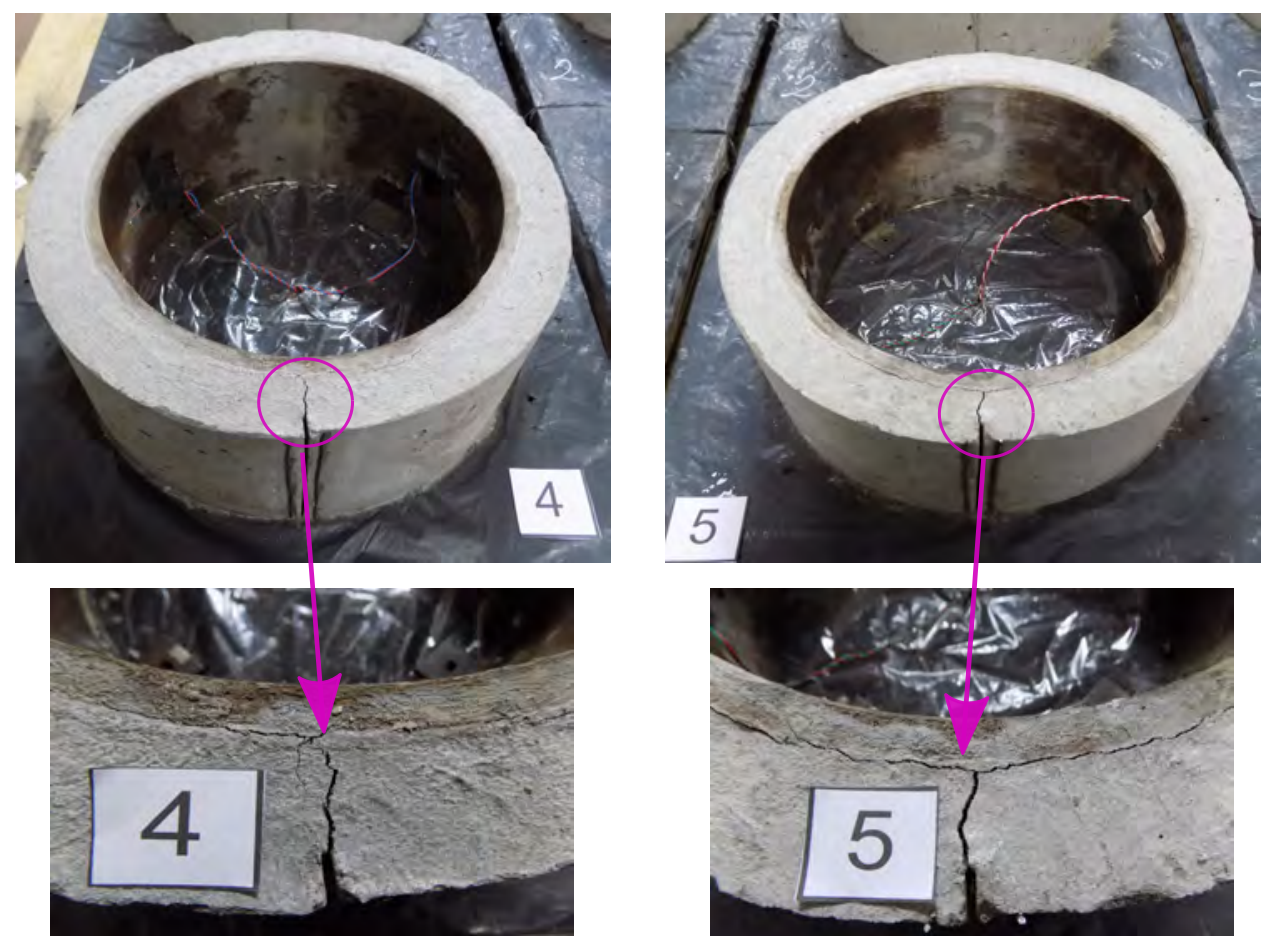

Figura 4.47: Ensayo de contracción restringida en anillos de hormigón con entalla- Fisuras observadas

\begin{tabular}{|c|c|}
\hline Número de anillo & Edad de fisuración [días] \\
\hline 3 & 7.02 \\
\hline 4 entallado & 7.02 \\
\hline 5 entallado & - \\
\hline 6 entallado & 7.03 \\
\hline
\end{tabular}

Tabla 4.16: Tiempos de aparición de fisuras obtenidos a partir de los registros continuos- Anillos entallados

La edad de fisuración obtenida para los anillos entallados a partir de la observación visual fue entre 4 y 7 días y la obtenida de los registros continuos fue de 7.03 días. El resultado obtenido del registro continuo se encuentra en el rango definido por el registro visual.

En esta etapa también se midió la apertura de las entallas de los anillos 4, 5 y 6 y de la fisura del anillo 3, mediante la utilización de un microscopio digital manual Dino-Lite Premier AM4113T, el cual permite obtener una imagen de calidad con un aumento superior a 200x.

Para la utilización del microscopio digital se realizó la calibración del 
mismo, empleando la escala de referencia aportada por el fabricante, y definiendo la separación entre puntos mediante una distancia conocida, ver Figura 4.48.
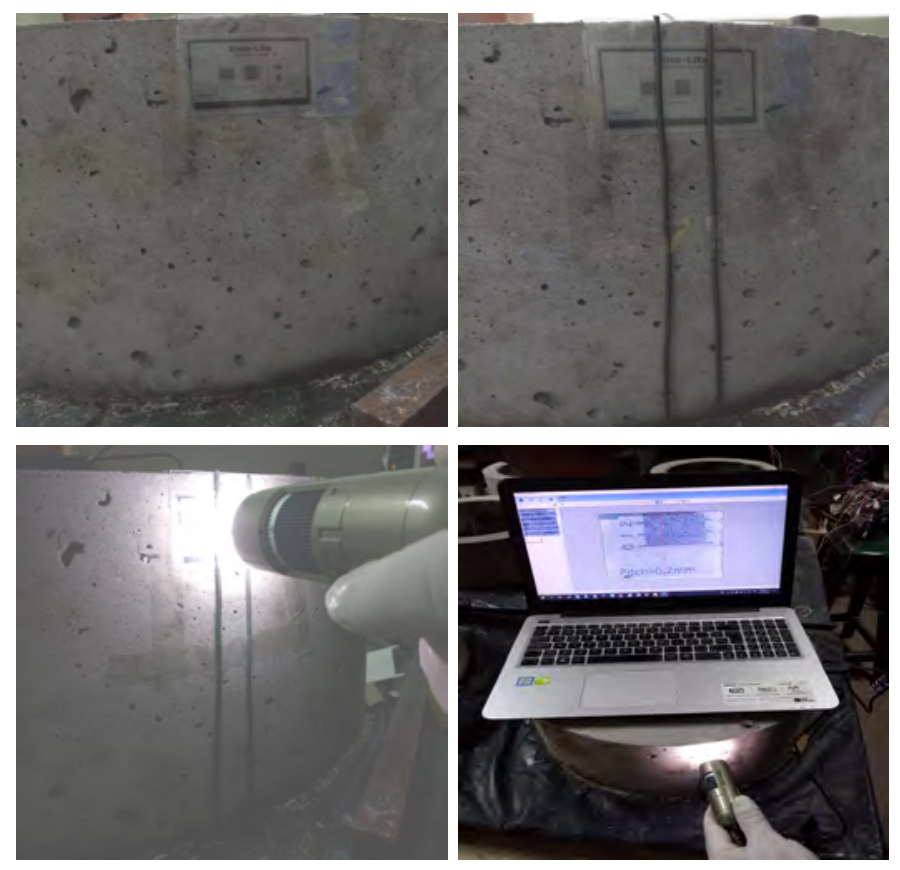

Figura 4.48: Proceso de calibración del microscopio digital utilizado para la medición de la apertura de las fisuras

En todos los casos se midieron las aperturas en tres puntos a lo largo del lateral de anillo.

En las Figuras 4.49, 4.50 y 4.51 se presentan los gráficos que muestran la evolución de la apertura de la entalla a lo largo del tiempo para los anillos 4, 5 y 6 y en la Figura 4.52 se muestra la evolución en el tiempo de la apertura de la fisura en lateral del anillo 3. En la Figura 4.53 se muestra la foto tomada para dos de los anillos.

A partir de los gráficos presentados puede observarse que para los anillos entallados, Figuras 4.49 y 4.51, las aperturas medidas en los tres puntos a distintas alturas, respecto de la base del anillo, tienden a los mismo valores. En cambio, en el anillo sin entalla, Figura 4.52, los valores de aperturas cambian para los puntos ubicados a distintas alturas. Esto es esperable, ya que la entalla es uniforme en todo el lateral del anillo, en cambio la fisura presenta un camino más errático. 


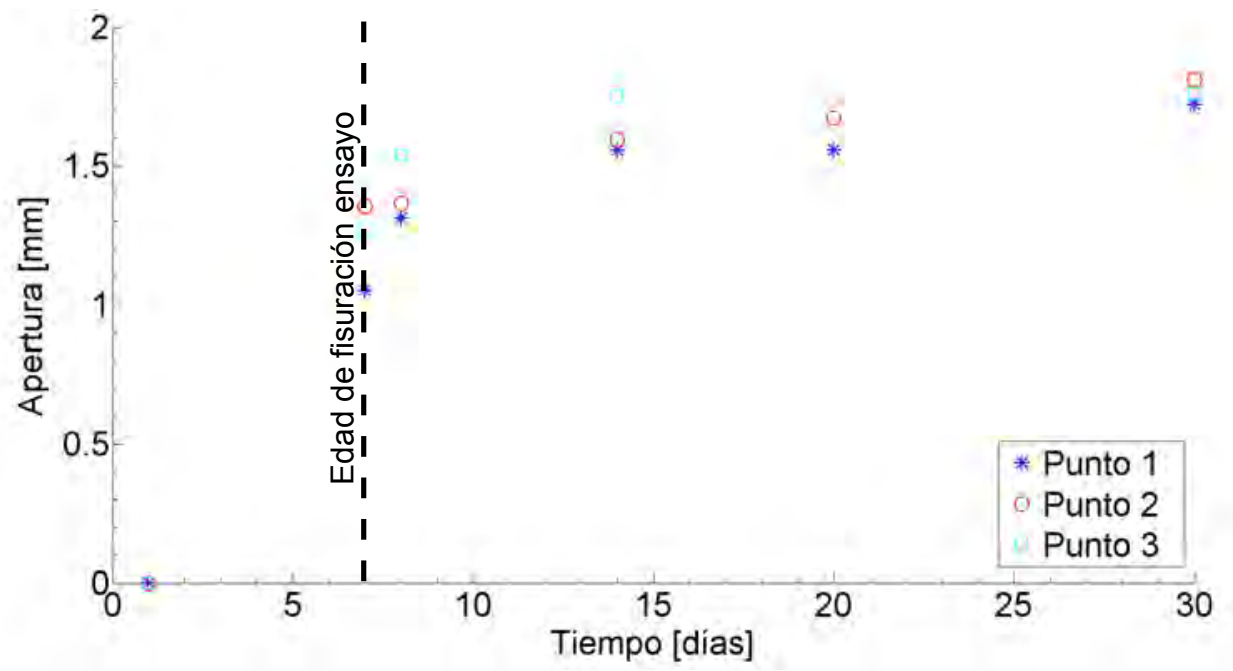

Figura 4.49: Apertura de la entalla medida en el lateral del anillo 4

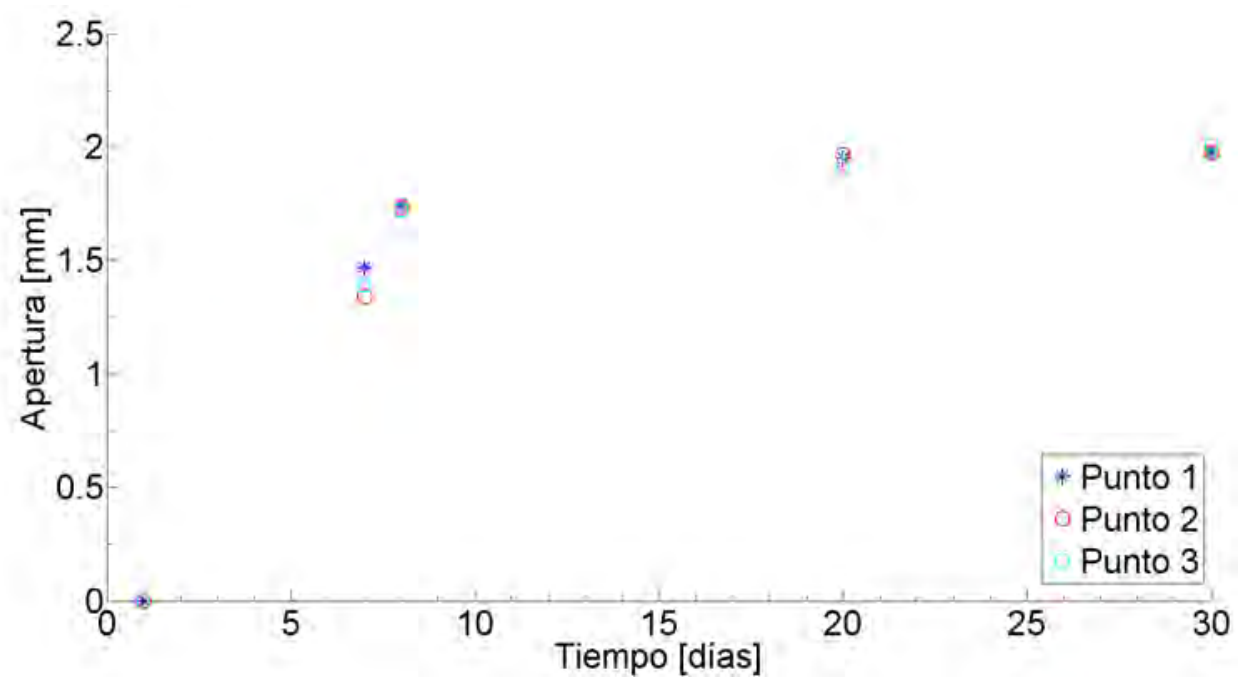

Figura 4.50: Apertura de la entalla medida en el lateral del anillo 5 


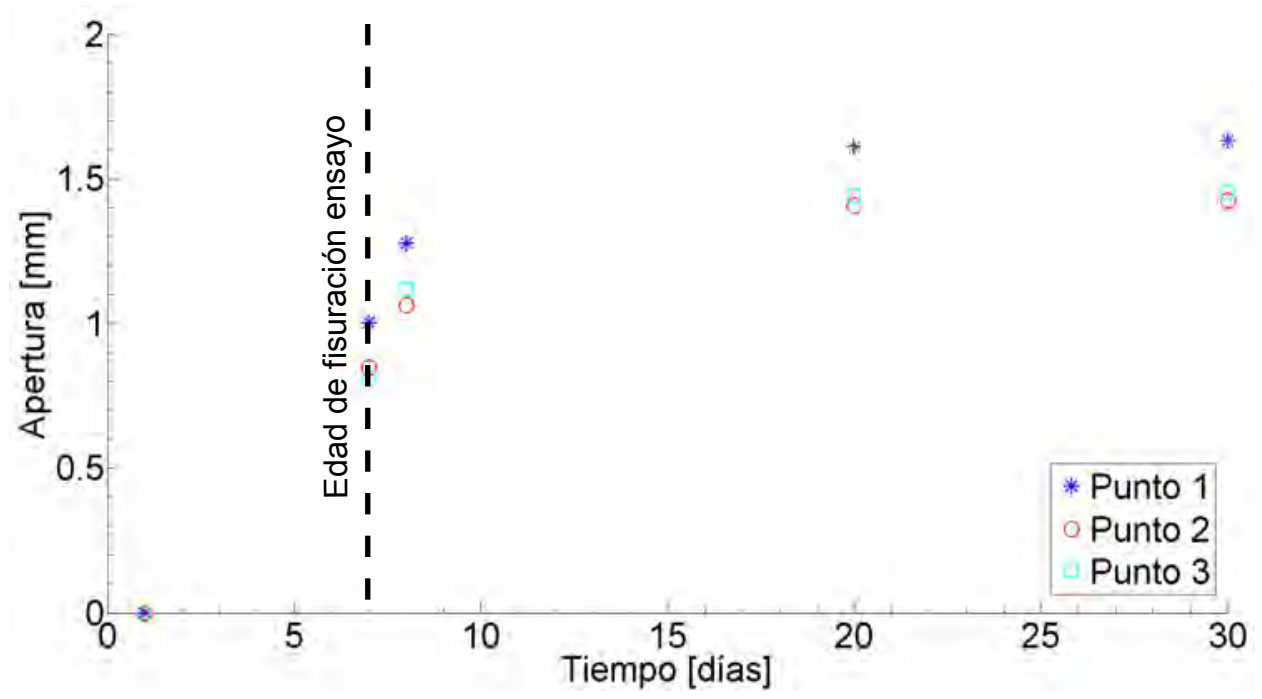

Figura 4.51: Apertura de la entalla medida en el lateral del anillo 6

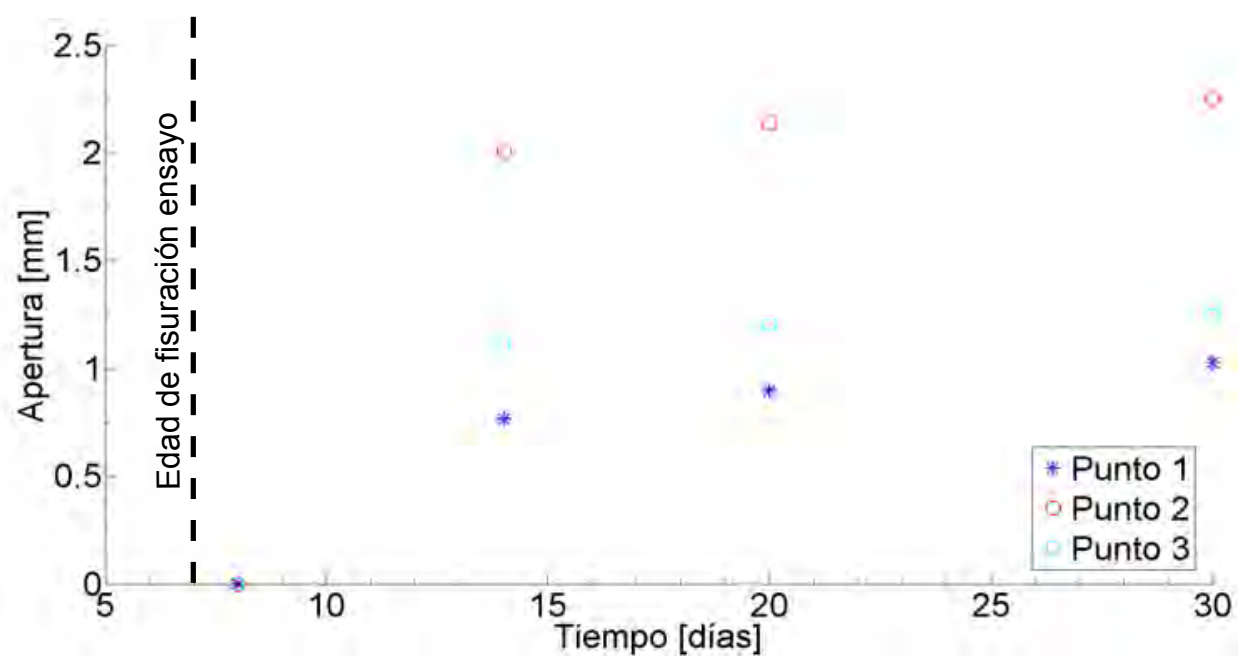

Figura 4.52: Apertura de la fisura medida en el lateral del anillo 3 


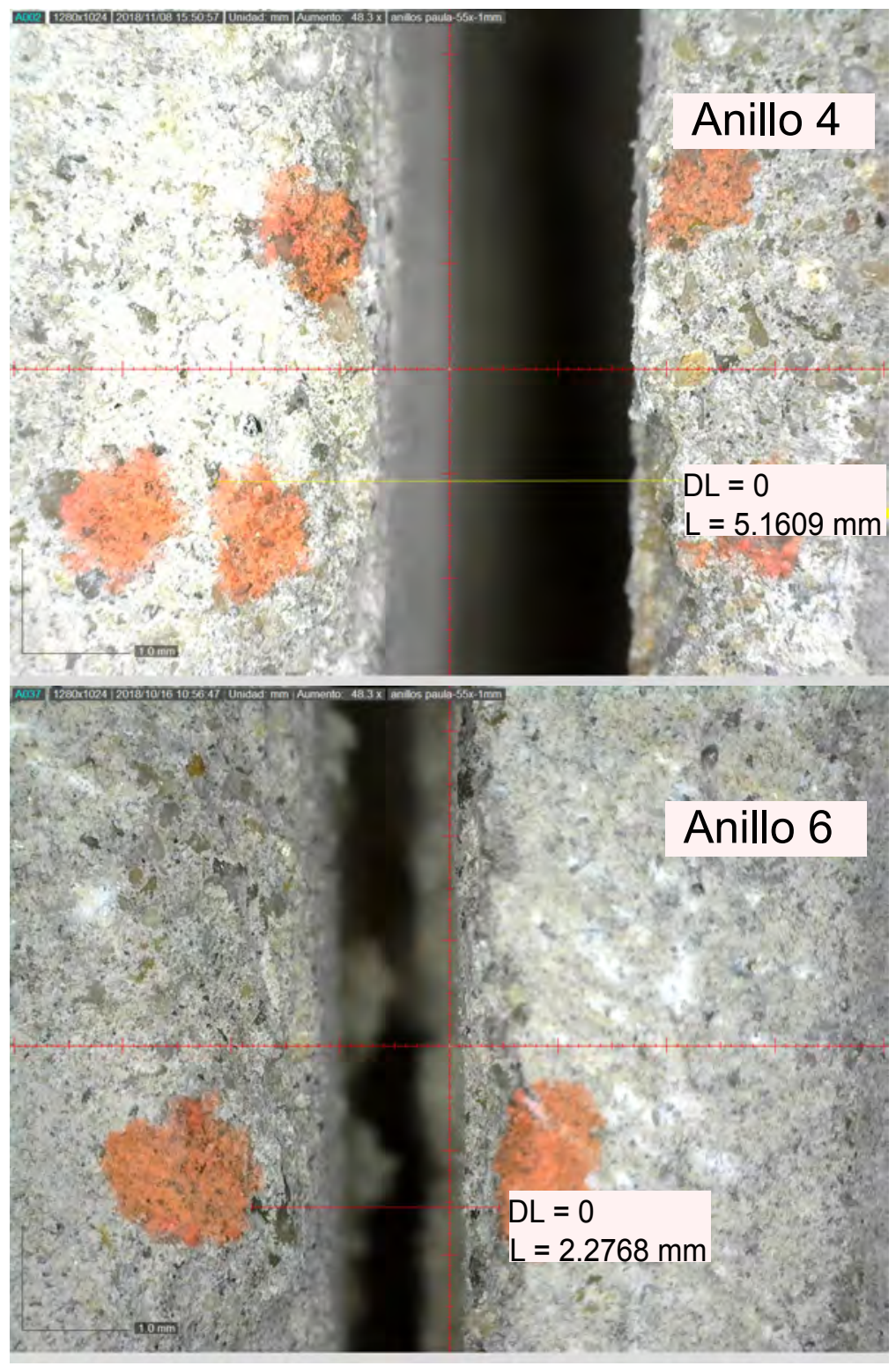

Figura 4.53: Fotografía para la medición de la apertura de la entalla en anillos 4 y 6 


\section{Capítulo 5}

\section{Validación numérica del algoritmo desarrollado}

\subsection{Introducción}

Para la validación del algoritmo desarrollado se simuló numéricamente el ensayo de contracción restringida en anillos de hormigón ASTM C 1581, ASTM (2004a), con el objetivo de predecir la edad de fisuración y comparar los resultados numéricos obtenidos con los resultados experimentales presentados en el Capítulo 4. Adicionalmente se simuló una modificación del ensayo considerando anillos elípticos en lugar de circulares para realizar la validación del lugar de aparición de la fisura, aspecto que no puede estudiarse en el caso de geometrías circulares debido a la simetría axial que presentan. Para la validación se utilizaron resultados experimentales disponibles en la biliografía, Dong et al. (2016).

\subsection{Validación del algoritmo desarrollado conside- rando resultados experimentales propios}

\section{Geometría}

\section{Anillo sin entalla}

En este caso se realizó un análisis numérico en dos dimensiones del ensayo ASTM C1581, ASTM (2004a). Simulando un anillo de hormigón de $405 \mathrm{~mm}$ de diámetro externo, $330 \mathrm{~mm}$ de diámetro interno y $37.5 \mathrm{~mm}$ de espesor, vinculado a un anillo de acero de $330 \mathrm{~mm}$ de diámetro externo y $13 \mathrm{~mm}$ de espesor, considerando un contacto sin fricción entre ambos anillos. Después de haber comparado distintos tamaños y tipos de elementos, se adoptaron dos tipos de mallados, cuadrado y triangular. La malla cuadrada cuenta con 7788 elementos tipo CPS4, elementos cuadrados lineales de cuatro nodos en 
tensión plana. Por otro lado, la malla triangular cuenta con 15576 elementos tipo CPS3, elementos triangulares lineales de tres nodos en tensión plana. En la Figura 5.1 se observan las mallas utilizadas.

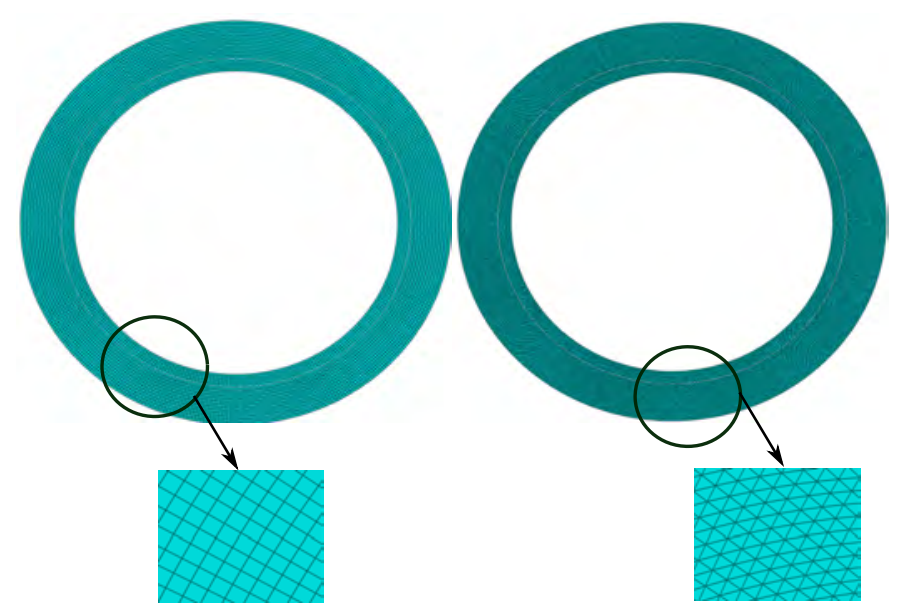

Figura 5.1: Mallas utilizadas en la simulación del ensayo de contracción restringida en anillos circulares

Se realizaron diez simulaciones con cada uno de los mallados considerando distribución aleatoria de las propiedades mecánicas del material. La duración del análisis fue de 24 días.

\section{Anillo con entalla}

Con el objetivo de romper la continuidad de la geometría circular se incorporó una entalla de $15 \mathrm{~mm}$ de largo en el anillo de hormigón analizado en la sección anterior. La entalla se modeló a través del comando seam, el cual permite generar una discontinuidad en el mallado. Se realizaron diez simulaciones considerando distribución aleatoria de las propiedades mecánicas del material. La duración del análisis fue de 24 días.

\section{Estado de cargas y propiedades mecánicas}

En ambos casos el estado de carga considerado fue una temperatura equivalente, determinada como se indicó en el Capítulo 4. Además en ese capítulo se presentaron las curvas de resistencia a compresión, tracción y módulo de elasticidad ajustado con el coeficiente obtenido a partir del ensayo de extensibilidad, que se utilizaron en las simulaciones numéricas.

Como se mencionó en el Capítulo 3, en el modelo de plasticidad con daño es necesario definir una ley de daño y una ley de ablandamiento. El valor de la apertura crítica a 28 días, $\left(w_{c 28}\right)$, obtenido a partir de la energía de fractura fue de $0.0795 \mathrm{~mm}$. El coeficiente de Poisson adopatado fue 0.2 . 
5.2. Validación del algoritmo desarrollado considerando resultados experimentales propios

En las simulaciones se utilizó la distribución de Weibull para el análisis aleatorio de las propiedades mecánicas del material. En este caso el parámetro de forma, $m$, adopta el valor 12, Zech y Wittmann (1977), y el parámetro de escala, $\sigma_{0}$, se obtuvo a partir de la ecuación 3.50, para un valor medio de la resistencia a la tracción de $3.52 \mathrm{MPa}$, dando como resultado un parámetro de escala de $3.65 \mathrm{MPa}$. Los valores de $V$ y de $V_{0}$ se adoptaron unitarios.

Para determinar el tamaño de las regiones en las que se asigna un determinado valor de la resistencia definida en la curva de Weibull, se considera que el mismo es igual a tres veces el tamaño máximo del agregado, como se indicó en el Capítulo 3. En este caso el tamaño máximo es $12 \mathrm{~mm}$, por lo que el tamaño de la región es de $36 \mathrm{~mm}$.

El valor del módulo de elasticidad del acero utilizado fue $210000 \mathrm{MPa}$ y el coeficiente de Poisson 0.3.

Tanto para el hormigón como para el acero se adoptó un coeficiente de contracción térmica igual a $1 x 10^{-5} 1 /{ }^{\circ} \mathrm{C}$.

\section{Determinación de la edad de fisuración}

\section{Anillo sin entalla}

Con el objetivo de establecer la edad de fisuración mediante el modelo numérico, se obtuvo la deformación circunferencial en cuatro elementos del anillo de acero ubicados en radios opuestos, los cuales se indican en la Figura 5.2. La edad de fisuración se determinó a partir de una discontinuidad en el gráfico de la deformación circunferencial a lo largo del tiempo. En la Figura 5.3 se indica la variación de la deformación circunferencial en el tiempo para uno de los anillos analizados, en donde se observa claramente el salto mencionado.

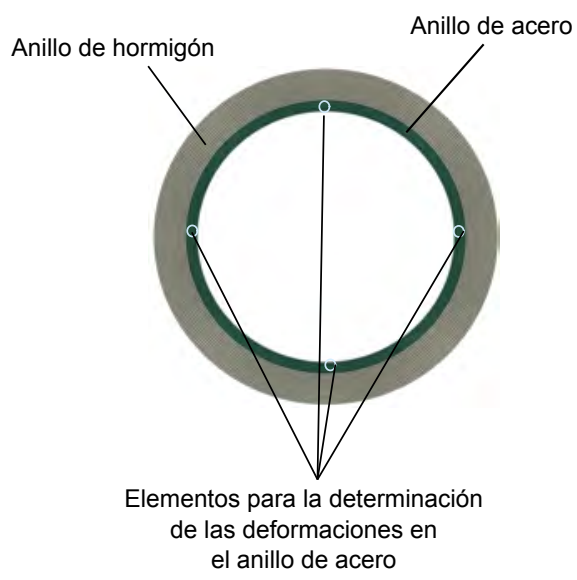

Figura 5.2: Elementos del anillo de acero en los que se miden las deformaciones circunferenciales 


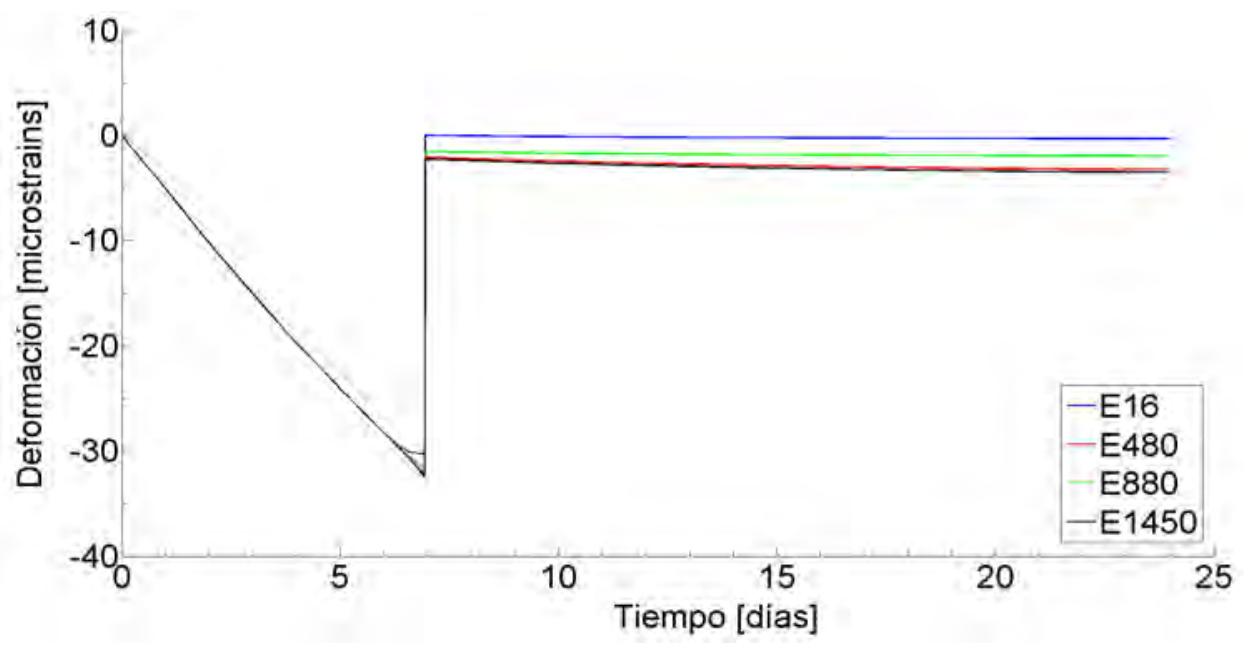

Figura 5.3: Variación de la deformación circunferencial en el tiempo para la simulación del ensayo de contracción restringida en anillos de hormigón

En las Tablas 5.1 y 5.2 se indican los resultados de las diez simulaciones de los anillos realizadas utilizando el mallado cuadrado y el mallado triangular, respectivamente.

\begin{tabular}{|c|c|}
\hline No de simulación & Tiempo de aparición de la fisura [días] \\
\hline 1 & 6.70 \\
\hline 2 & 7.14 \\
\hline 3 & 6.60 \\
\hline 4 & 7.76 \\
\hline 5 & 7.08 \\
\hline 6 & 7.61 \\
\hline 7 & 7.88 \\
\hline 8 & 7.90 \\
\hline 9 & 6.95 \\
\hline 10 & 9.19 \\
\hline Promedio & 7.48 \\
\hline Desviación estándar & 0.77 \\
\hline Promedio corregido & 7.68 \\
\hline
\end{tabular}

Tabla 5.1: Tiempo de aparición de la fisura en anillos circulares con mallado cuadrado

En ambos casos la corrección del promedio se refiere a la incorporación del tiempo de fraguado en el resultado final, ya que se considera que la simulación comienza luego del fraguado del hormigón. El tiempo de fraguado inicial se presentó en el Capítulo 4, y fue de 4.8 hs. 
5.2. Validación del algoritmo desarrollado considerando resultados experimentales propios

\begin{tabular}{|c|c|}
\hline $\mathrm{N}^{\mathbf{O}}$ de simulación & Tiempo de aparición de la fisura [días] \\
\hline 1 & 7.64 \\
\hline 2 & 7.39 \\
\hline 3 & 8.13 \\
\hline 4 & 7.47 \\
\hline 5 & 9.26 \\
\hline 6 & 7.63 \\
\hline 7 & 6.82 \\
\hline 8 & 6.93 \\
\hline 9 & 7.71 \\
\hline 10 & 4.33 \\
\hline Promedio & 7.33 \\
\hline Desviación estándar & 1.25 \\
\hline Promedio corregido & 7.53 \\
\hline
\end{tabular}

Tabla 5.2: Tiempo de aparición de la fisura en anillos circulares con mallado triangular

En las Tablas 5.3 y 5.4 se muestra la comparación de los resultados experimentales presentados en el Capítulo 4 con los resultados numéricos obtenidos con los mallados cuadrado y triangular, respectivamente. Se observa que los resultados obtenidos numéricamente se aproximan a los obtenidos experimentalmente.

\begin{tabular}{|c|c|c|}
\hline \multicolumn{2}{|c|}{ Resultados experimentales } & Resultados numéricos \\
\hline Visual [Días] & Registro [Días] & Malla cuadrada [Días] \\
\hline $7-8$ & 7.05 & 7.68 \\
\hline
\end{tabular}

Tabla 5.3: Comparación resultados experimentales y numéricos para el mallado cuadrado

\begin{tabular}{|c|c|c|}
\hline \multicolumn{2}{|c|}{ Resultados experimentales } & Resultados numéricos \\
\hline Visual [Días] & Registro [Días] & Malla triangular [Días] \\
\hline $7-8$ & 7.05 & 7.53 \\
\hline
\end{tabular}

Tabla 5.4: Comparación resultados experimentales y numéricos para el mallado triangular

\section{Anillo con entalla}

En las secciones anteriores se explicó cómo se calcula el tiempo de aparición de las fisuras en el modelo numérico, por lo que en la Tabla 5.5 se 
indican los resultados obtenidos para el anillo entallado. Se utilizó un mallado cuadrado, ya que en el caso de los anillos sin entallas se observó que los resultados promedio de las simulaciones para ambos tipo de mallado (cuadrado y triangular) eran similares.

\begin{tabular}{|c|c|}
\hline No $^{\mathbf{O}}$ de simulación & Tiempo de aparición de la fisura [días] \\
\hline 1 & 5.51 \\
\hline 2 & 5.33 \\
\hline 3 & 6.21 \\
\hline 4 & 6.65 \\
\hline 5 & 6.31 \\
\hline 6 & 5.35 \\
\hline 7 & 6.13 \\
\hline 8 & 6.47 \\
\hline 9 & 6.58 \\
\hline 10 & 6.46 \\
\hline Promedio & 6.10 \\
\hline Desviación estándar & 0.51 \\
\hline Promedio corrección & 6.30 \\
\hline
\end{tabular}

Tabla 5.5: Tiempo de aparición de la fisura en anillos circulares entallados simulados numéricamente

Si se comparan el tiempo promedio de aparición de la fisura en el caso del anillo entallado, Tabla 5.5, con el del anillo sin entalla, Tablas 5.1 y 5.2 , se observa que el tiempo de aparición en el primer caso es menor, lo cual puede relacionarse con la reducción del ligamento en esa zona del anillo de hormigón debido a la presencia de la entalla, siendo por tanto menor la sección resistente.

En la Tabla 5.6 se muestra la comparación de los resultados experimentales presentados en el Capítulo 4 con los resultados numéricos.

\begin{tabular}{|c|c|c|}
\hline \multicolumn{2}{|c|}{ Resultados experimentales } & Resultados numéricos \\
\hline Visual [Días] & Registro [Días] & Malla [Días] \\
\hline $4-7$ & 7.03 & 6.30 \\
\hline
\end{tabular}

Tabla 5.6: Comparación resultados experimentales y numéricos para el anillo entallado

En las Figuras 5.4, 5.5 y 5.6, se indican las edades de fisuración para los anillos con entalla (4 y 6 ) y sin entalla (anillo 3 ) obtenidas a partir de los ensayos presentados en el Capítulo 4 en línea de puntos, y las obtenidas numericamente en rosado, respectivamente. También se muestran los valores de apertura medidos en el ensayo. 
5.2. Validación del algoritmo desarrollado considerando resultados experimentales propios

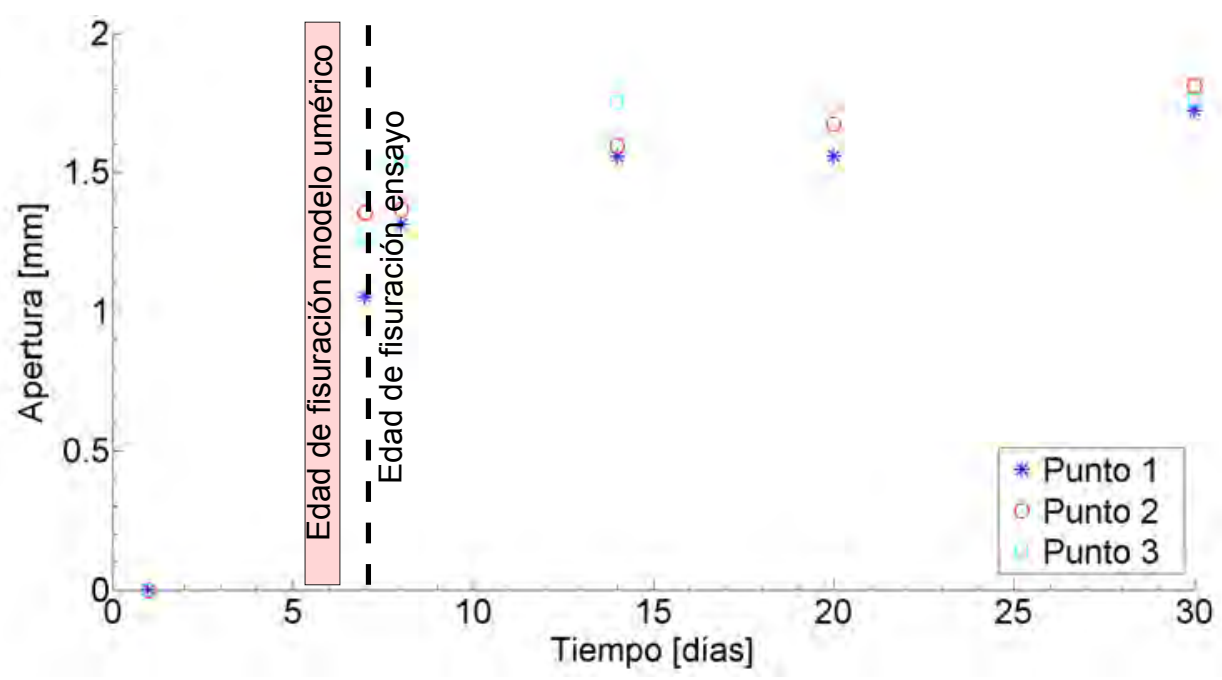

Figura 5.4: Comparación edades de fisuración experimental y numéricaAnillo 4 con entalla

Se observa que en el anillos 3 la edad de fisuración medida en el ensayo se encuentra en el rango de edades obtenidas con el modelo numérico. En los anillos 4 y 6 los resultados experimentales se encuentran por debajo de las edades de fisuración obtenidas numéricamente.

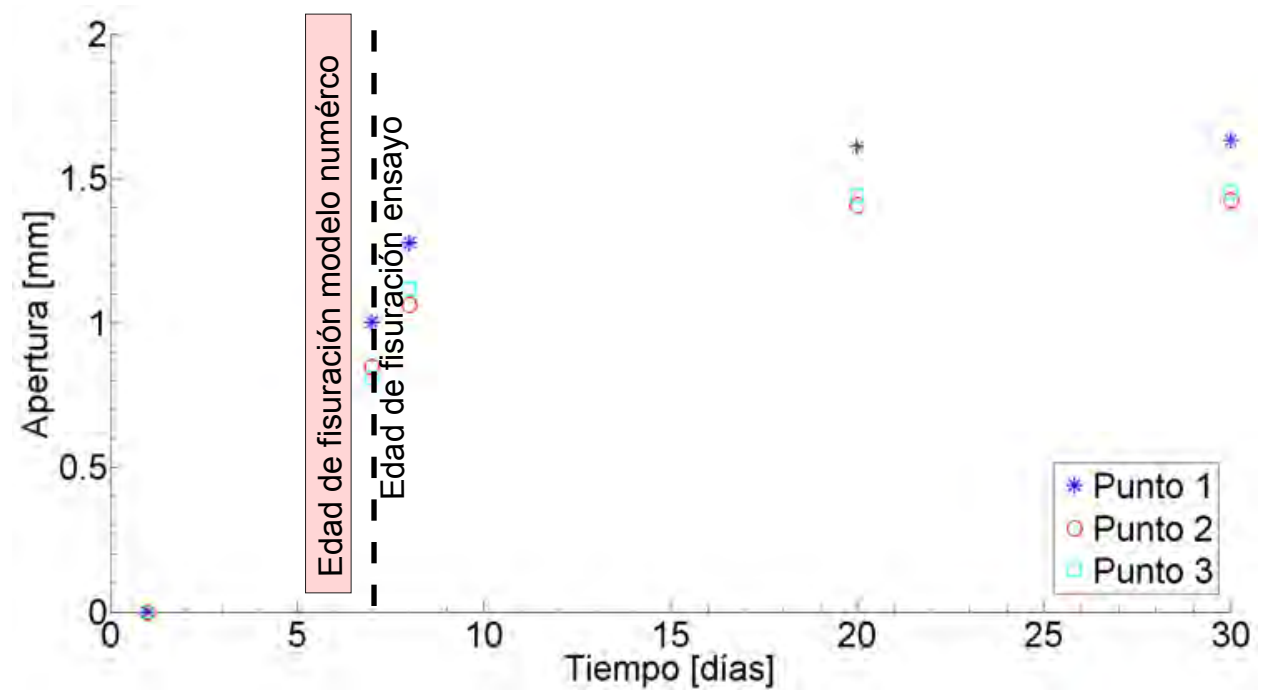

Figura 5.5: Comparación edades de fisuración experimental y numéricaAnillo 6 con entalla 


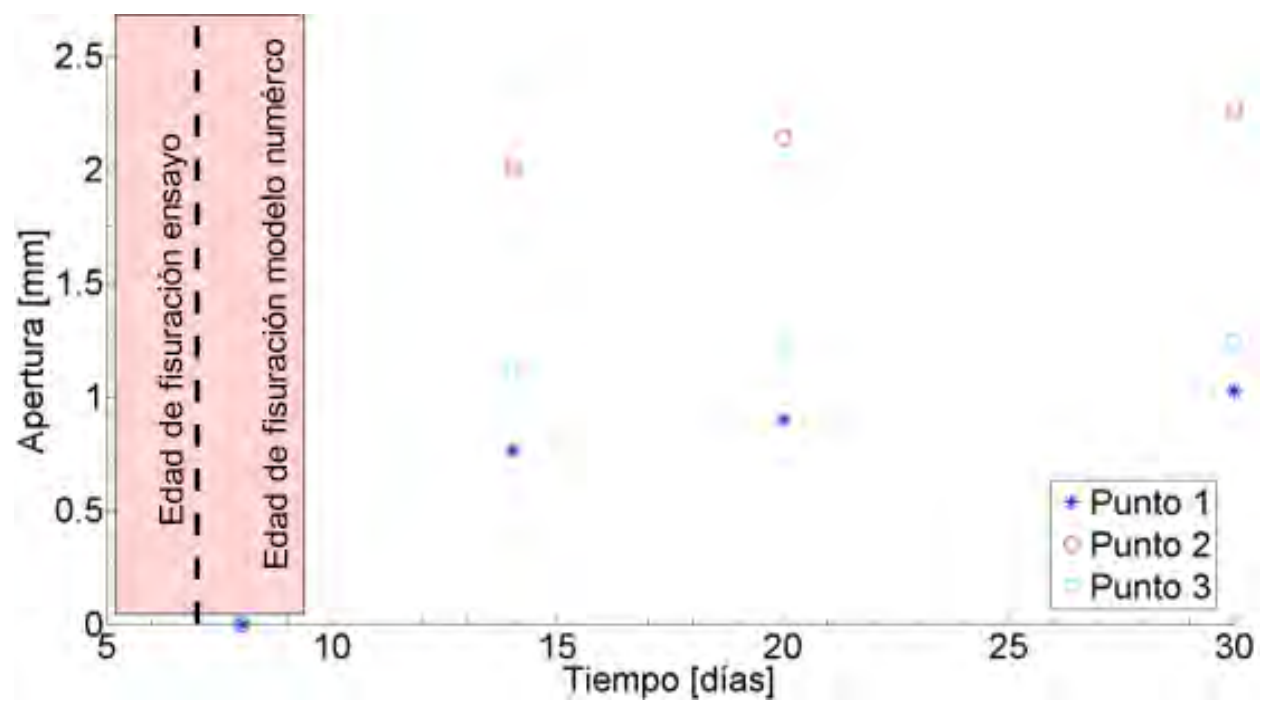

Figura 5.6: Comparación edades de fisuración experimental y numéricaAnillo 3 sin entalla

\subsection{Validación del algoritmo desarrollado a partir de los resultados de otros autores}

Se analizaron un anillo circular y dos anillos elípticos bajo contracción restringida, cuyas geometrías y dimensiones se indican en la Figura 5.7 y en la Tabla 5.7, Dong et al. (2016). Se utilizaron dos tipos de elementos, cuadriláteros y triangulares aproximadamente equiláteros, como se muestra en la Figura 5.8.

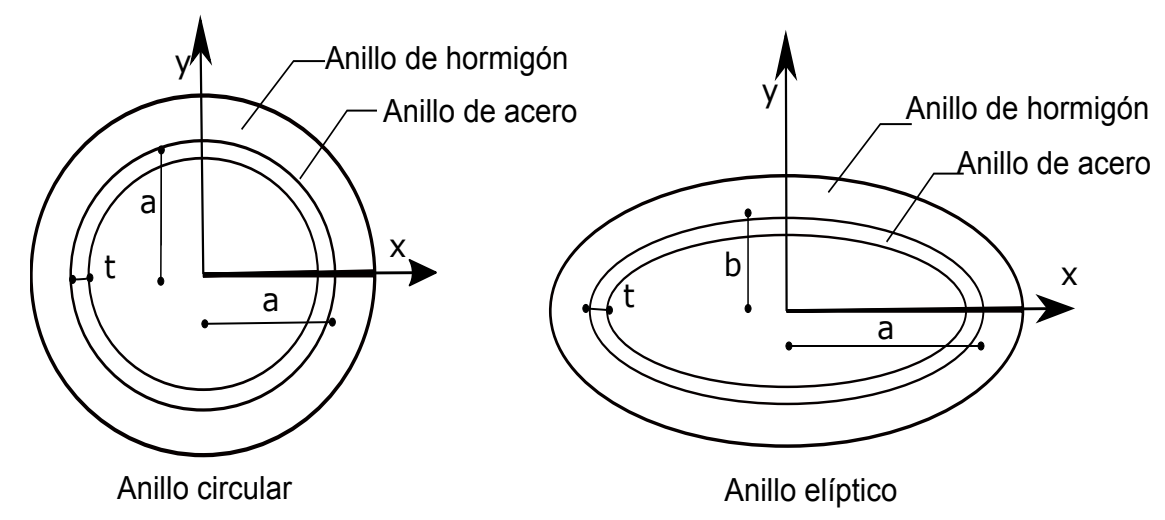

Figura 5.7: Geometría de los anillos analizados

Con el objetivo de determinar la influencia del tamaño de los elementos en los resultados obtenidos, se compararon dos tamaños, tanto para los elementos cuadriláteros como para los triangulares. Estos tamaños se indican 
5.3. Validación del algoritmo desarrollado a partir de los resultados de otros autores

\begin{tabular}{|c|c|c|}
\hline \multirow{2}{*}{ Anillo } & \multicolumn{2}{|c|}{ Dimensión [mm] } \\
\cline { 2 - 3 } & $\mathrm{a}$ & $\mathrm{b}$ \\
\hline 1 & 150 & - \\
\hline 2 & 150 & 125 \\
\hline 3 & 150 & 100 \\
\hline
\end{tabular}

Tabla 5.7: Dimensiones de los anillos circulares y elípticos analizados

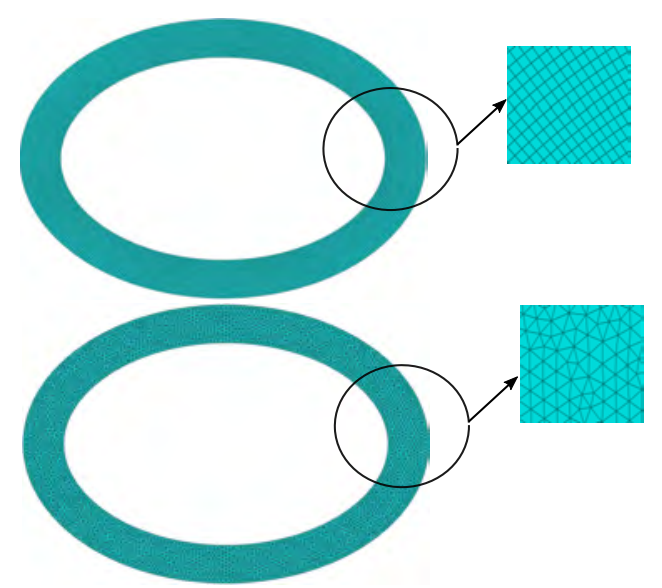

Figura 5.8: Mallados utilizados en los anillos elípticos analizados

en las Tablas 5.8 y 5.9 .

\begin{tabular}{|c|c|c|}
\hline \multirow{2}{*}{ Anillo } & \multicolumn{2}{|c|}{ Longitud de lado del elemento cuadrado [mm] } \\
\cline { 2 - 3 } & Mallado fino & Mallado grueso \\
\hline 1 & 2.8 & 10 \\
\hline 2 & 2.5 & 10 \\
\hline 3 & 2.4 & 10 \\
\hline
\end{tabular}

Tabla 5.8: Tamaños de elementos utilizados para analizar su efecto en los resultados obtenidos-Mallado cuadrado

\begin{tabular}{|c|c|c|}
\hline \multirow{2}{*}{ Anillo } & \multicolumn{2}{|c|}{ Longitud de lado del elemento triangular [mm] } \\
\cline { 2 - 3 } & Mallado fino & Mallado grueso \\
\hline 1 & 4.8 & 10 \\
\hline 2 & 4.1 & 10 \\
\hline 3 & 3.7 & 10 \\
\hline
\end{tabular}

Tabla 5.9: Tamaños de elementos utilizados para analizar su efecto en los resultados obtenidos-Mallado triangular

Para la obtención de los archivos de entrada que utiliza Abaqus/Standard 
en las simulaciones numéricas se desarrolló un algoritmo en Python logrando de esta forma agilizar el proceso de generación de las geometrías y estados de carga.

Para cada una de las geometrías se realizó un análisis elástico lineal (ML) y un análisis aplicando el modelo de plasticidad con daño presentado en el Capítulo 3 (CDP), considerando en este último caso distribución uniforme (CDP-U) y distribución aleatoria de las propiedades mecánicas del material (CDP-A), para diez asignaciones aleatorias de propiedades. El objetivo de esto último consiste en otorgarle un enfoque estadístico al análisis de los resultados obtenidos calculando valores medios y la desviación estándar de los mismos. Se adoptó esta cantidad de asignaciones porque de los análisis realizados surge que a partir de este número las dispersiones en los resultados obtenidos dejan de presentar variaciones.

Los anillos se modelaron en dos dimensiones, definiendo un contacto entre los anillos de hormigón y acero sin fricción. La duración del análisis fue de 28 días.

El estado de cargas aplicado al modelo consiste en una temperatura equivalente obtenida a partir de un ensayo de contracción libre en probetas prismáticas de hormigón de $7 \times 7 \times 30 \mathrm{~cm}$ con diferente relación $A / V$ (área expuesta/volumen total) con el objetivo de simular diferentes condiciones de exposición de acuerdo a la metodología propuesta por Dong et al. (2016) y explicada en el Capítulo 4.

En la Figura 5.9 se presentan las curvas obtenidas a partir del ensayo y su transformación a temperatura equivalente, Dong et al. (2016). En anillos delgados como el que fue analizado, la contracción puede considerarse uniforme en la dirección radial, Moon et al. (2006); Dong et al. (2013).

Tanto la resistencia a tracción, como a compresión y el módulo de elasticidad son variables en el tiempo. Las ecuaciones que representan la variación en el tiempo de las mencionadas propiedades se obtuvieron a partir de los resultados experimentales presentados por Dong et al. (2016) y se indican a continuación:

$$
\begin{gathered}
E(t)=16940,2 *\left(\exp ^{0,28 *\left(1-(20 / t)^{0,5}\right.}\right)^{0,5} \\
f_{c}(t)=38 * \exp ^{\left(0,28(1-28 / t)^{0,5}\right)} \\
f_{c t}(t)=1,3 *\left(\left(f_{c} / 12\right)^{1 / 3}\right)^{1,45}+1,45
\end{gathered}
$$

Como se explicó en capítulos anteriores, para implementar un modelo de plasticidad con daño es necesario definir una ley de daño y una función de ablandamiento. En este caso se adoptó una función de ablandamiento lineal, la cual como se explicó en el Capítulo 2 resulta adecuada para el hormigón cuando no se tiene demasiada información sobre el comportamiento 
5.3. Validación del algoritmo desarrollado a partir de los resultados de otros autores

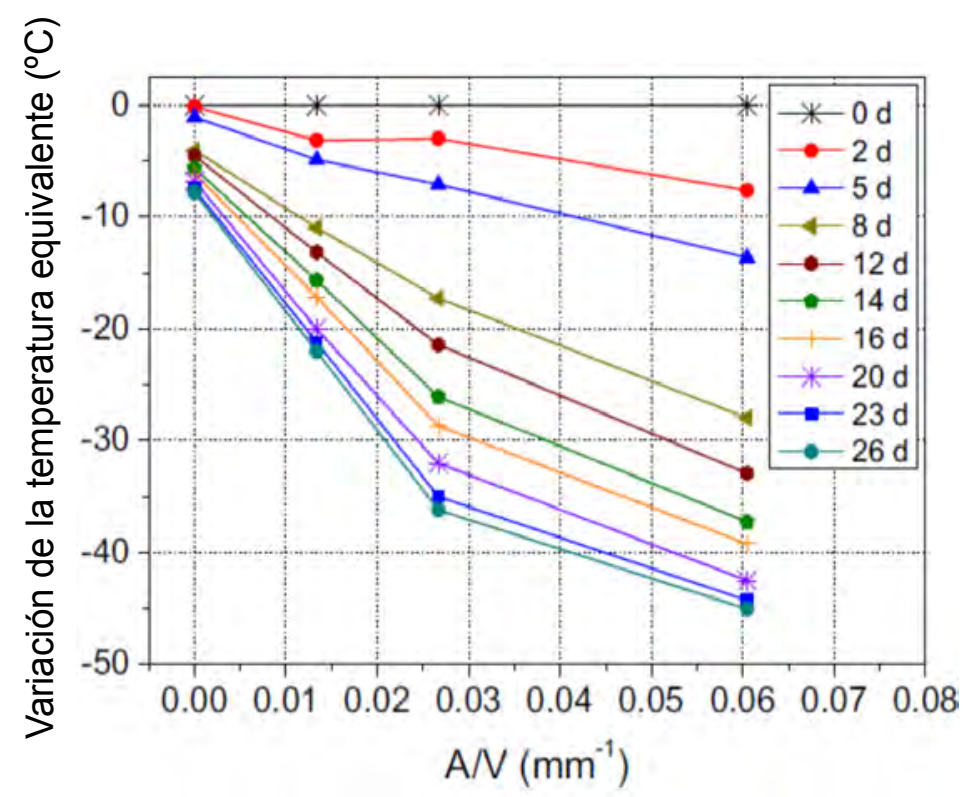

Figura 5.9: Temperatura equivalente utilizada en las simulaciones, Dong et al. (2016)

del material. Aunque sobre estima los valores obtenidos, al utilizarla los resultados se encuentran del lado seguro. La apertura crítica, $\left(w_{c}\right)$, se calculó a partir de la energía de fractura $\left(G_{f}\right)$, estimada para el hormigón en estudio en $0.075 \mathrm{Nmm} / \mathrm{mm}^{2}$, obteniéndose un valor de $0.0655 \mathrm{~mm}$. Para el hormigón el módulo de elasticidad se redujo un $20 \%$ con el objetivo de considerar el efecto del creep, Dong et al. (2016); Moon et al. (2006) y se adoptó un coeficiente de Poisson de 0.2. En el caso del acero el valor del módulo de elasticidad utilizado fue $210000 \mathrm{MPa}$ y el coeficiente de Poisson 0.3.

En estas simulaciones se utilizó la distribución de Weibull para el análisis aleatorio de las propiedades del material adoptando para el parámetro de forma $m$ el valor 12 , Zech y Wittmann (1977), y el parámetro de escala, $\sigma_{0}$, se obtuvo a partir de la ecuación 3.50, para un valor medio de la resistencia a compresión de $38 \mathrm{MPa}$, obteniéndose un factor de escala de $39.46 \mathrm{MPa}$. Los valores de $V$ y de $V_{0}$ se adoptaron unitarios.

Tanto para el hormigón como para el acero se adoptó un coeficiente de contracción térmica igual a $1 \times 10^{-5} 1 /{ }^{\circ} \mathrm{C}$.

\subsubsection{Determinación de la edad de fisuración}

Como se explicó anteriormente, para determinar la edad de fisuración en el modelo numérico, se obtuvo la deformación circunferencial en cuatro elementos del anillo de acero ubicados en radios opuestos, ver Figura 5.2. La 
edad de fisuración se determina a partir de una discontinuidad en el gráfico de la deformación circunferencial a lo largo del tiempo.

En las Tablas 5.10 y 5.11 se presentan las edades de aparición de fisuras obtenidas a partir de las simulaciones realizadas y de los resultados experimentales presentados en Dong et al. (2016), para el mallado fino con elementos cuadriláteros y triangulares, respectivamente. Por otro lado, en las Tablas 5.12 y 5.13 se presentan los mismos resultados pero para el mallado grueso de elementos cuadriláteros y triangulares. En la primera columna se indica la identificación de cada uno de los anillos; el número (1, 2 o 3 ) hace referencia a los anillos indicados en la Tabla 5.7, las letras G y F refieren al mallado (fino o grueso) y las letras $\mathrm{C}$ y $\mathrm{T}$ al tipo de elemento (cuadrado o triangular).

Comparando los resultados de las Tablas 5.10 y 5.12 se observa que al considerar propiedades mecánicas uniformes, el anillo 1 no presenta fisuras con el mallado grueso pero si al utilizar el mallado fino. Sin embargo en los anillos de 2 y 3 sucede lo contrario, ya que presentan fisuras con el mallado grueso y no con el mallado fino. Esto permite señalar la existencia de una dependencia con el tamaño de malla en los resultados al utilizar el modelo de plasticidad con daño con distribución uniforme de las propiedades mecánicas del material.

\begin{tabular}{|c|c|c|c|c|}
\hline Anillo & Ensayo [Días] & ML [Días] & CDP-U [Días] & CDP-A [días] \\
\hline 1-F-C & 14.50 & 13.00 & 25.91 & 13.70 \\
\hline 2-F-C & 14.50 & 13.00 & NO FISURA & 14.40 \\
\hline 3-F-C & 14.50 & 12.82 & NO FISURA & 14.60 \\
\hline
\end{tabular}

Tabla 5.10: Edad de fisuración obtenida en simulaciones realizadas en anillos con mallado cuadrado-Mallado fino

\begin{tabular}{|c|c|c|c|c|}
\hline Anillo & Ensayo [Días] & ML [Días] & CDP-U [Días] & CDP-A [días] \\
\hline 1-F-T & 14.50 & 13.00 & 16.52 & 14.80 \\
\hline 2-F-T & 14.50 & 13.00 & 17.48 & 15.10 \\
\hline 3-F-T & 14.50 & 10.32 & 16.78 & 15.90 \\
\hline
\end{tabular}

Tabla 5.11: Edad de fisuración obtenida en simulaciones realizadas en anillos con mallado triangular-Mallado fino

Si se comparan nuevamente los resultados de las Tablas 5.10 y 5.12 , pero considerando distribución aleatoria de las propiedades mecánicas en el modelo de plasticidad con daño, se observa en primer lugar que todos los anillos con los dos tamaños de elementos se fisuran. En segundo lugar las diferencias porcentuales entre las edades de fisuración calculadas con el mallado fino y el mallado grueso son: $5.3 \%, 3.4 \%$ y $1.8 \%$, para los anillos 1,2 y 3 respectivamente. Por este motivo se evidencia una reducción de la 
5.3. Validación del algoritmo desarrollado a partir de los resultados de otros autores

\begin{tabular}{|c|c|c|c|c|}
\hline Anillo & Ensayo [Días] & ML [Días] & CDP-U [Días] & CDP-A [días] \\
\hline 1-G-C & 14.50 & 13.50 & No FISURA & 14.50 \\
\hline 2-G-C & 14.50 & 13.50 & 16.00 & 14.90 \\
\hline 3-G-C & 14.50 & 11.32 & 16.38 & 14.90 \\
\hline
\end{tabular}

Tabla 5.12: Edad de fisuración obtenida en simulaciones realizadas en anillos con mallado cuadrado-Mallado grueso

\begin{tabular}{|c|c|c|c|c|}
\hline Anillo & Ensayo [Días] & ML [Días] & CDP-U [Días] & CDP-A [días] \\
\hline 1-G-T & 14.50 & 12.50 & 15.47 & 14.70 \\
\hline 2-G-T & 14.50 & 13.00 & 16.47 & 15.50 \\
\hline 3-G-T & 14.50 & 11.82 & 16.56 & 15.80 \\
\hline
\end{tabular}

Tabla 5.13: Edad de fisuración obtenida en simulaciones realizadas en anillos con mallado triangular-Mallado grueso

dependencia de la malla del modelo de plasticidad con daño al considerar un material con distribución aleatoria de las propiedades mecánicas.

Si el análisis realizado anteriormente se repite para el mallado triangular, Tablas 5.11 y 5.13 , se observa que aparecen fisuras tanto para el mallado fino como para el mallado grueso con distribución uniforme así como con distribución aleatoria de las propiedades mecánicas del material. Sin embargo si se analizan las diferencias porcentuales de las edades de aparición de fisuras entre el mallado fino y el mallado grueso, tanto al considerar las propiedades mecánicas distribuidas de manera uniforme como aleatoria, se observa que en el primer caso estas diferencias son del $6.4 \%, 5.8 \%$ y $1.3 \%$, para los anillos 1, 2 y 3 respectivamente. Sin embargo, en el segundo caso esas diferencias se reducen a $0.56 \%, 2.18 \%$ y $0.13 \%$, para los anillos 1,2 y 3 , respectivamente. Por lo que puede evidenciarse la fuerte reducción en la dependencia con el tamaño de malla de los resultados al aplicar distribución aleatoria de las propiedades mecánicas. Además al comparar la utilización de un mallado triangular y uno cuadrado, si se considera la distribución aleatoria de las propiedades mecánicas, en el primero se observa un menor efecto del tamaño de la malla en los resultados obtenidos.

Se realizó la comparación de los resultados experimentales presentados por Dong et al. (2016) con los obtenidos utilizando el modelo elástico lineal, y el modelo de plasticidad con daño con distribución de propiedades mecánicas uniformes y con distribución de propiedades mecánicas aleatorias. Se consideraron las simulaciones realizadas con el mallado fino tanto para elementos cuadrados como triangulares.

En la Tabla 5.10 puede observarse que para todos los casos la menor diferencia obtenida entre los resultados experimentales y los numéricos corresponden a las simulaciones realizadas con el modelo de plasticidad con 
daño con distribución aleatoria de las propiedades mecánicas del material. Los resultados de las simulaciones lineales presentan diferencias considerables en relación a los resultados experimentales como era de esperarse, lo cual justifica la necesidad de utilizar modelos no lineales en los problemas analizados en la presente Tesis.

Al aplicar el modelo de plasticidad con daño con distribución aleatoria de propiedades la edad de fisuración presentada es el promedio de las diez distribuciones aleatorias analizadas, además se calcularon las desviaciones estándar para cada uno de los anillos con cada tipo de mallado, siendo de $0.7,0.8$ y 0.7 días para los anillos 1,2 y 3 con mallado cuadrado y $0.2,0.7$ y 0.6 días para los anillos 1,2 y 3 con mallado triangular.

Para los casos en los que se utilizó el modelo de plasticidad con daño con propiedades uniformes, las fisuras aparecieron únicamente en el anillo circular, los anillos elípticos permanecieron sin daño, como se indicó anteriormente.

\subsubsection{Zonas dañadas}

Los modelos elásticos lineales no permiten predecir la localización de fisuras, como así tampoco los modelos no lineales aplicados a geometrías circulares, debido a la simetría axial de las mismas. Por el contrario, en las geometrías elípticas, es posible localizar las fisuras, ya que en este caso se presentan dos ejes de simetría diferentes. En la Figura 5.10 se observan las fisuras obtenidos experimentalmente para el anillo 2.

Las Figuras 5.11 y 5.12 presentan las zonas dañadas para el anillo elíptico 2 con distribución aleatoria de las propiedades mecánicas utilizando mallados con elementos cuadrados y triangulares.

Los resultados experimentales muestran que las fisuras aparecen cerca de los radios menores, lo cual se verifica, para el caso de distribución aleatoria de las propiedades mecánicas.
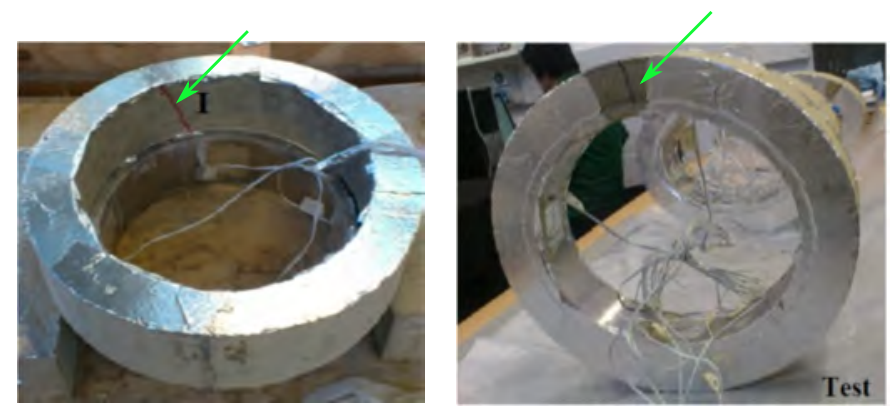

Figura 5.10: Ubicación de las fisuras en ensayos experimentales para el anillo 2, Dong et al. (2016) 
5.3. Validación del algoritmo desarrollado a partir de los resultados de otros autores

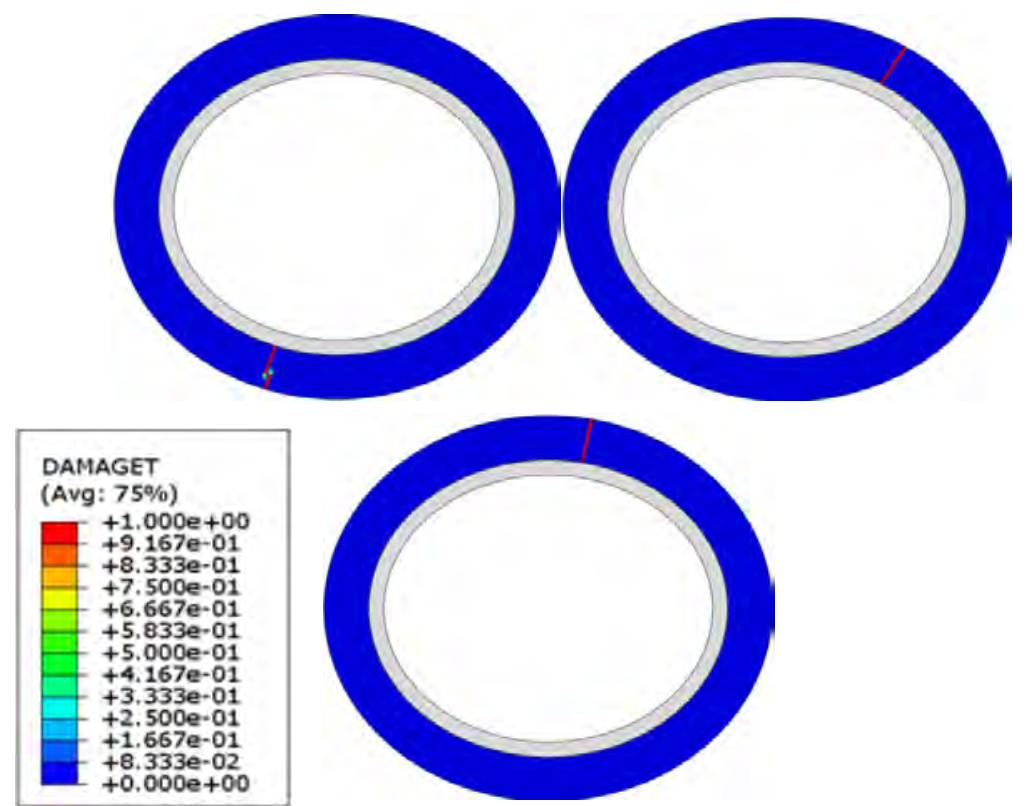

Figura 5.11: Zonas dañadas para simulaciones realizadas con mallado cuadrado y material aleatorio para el anillo 2

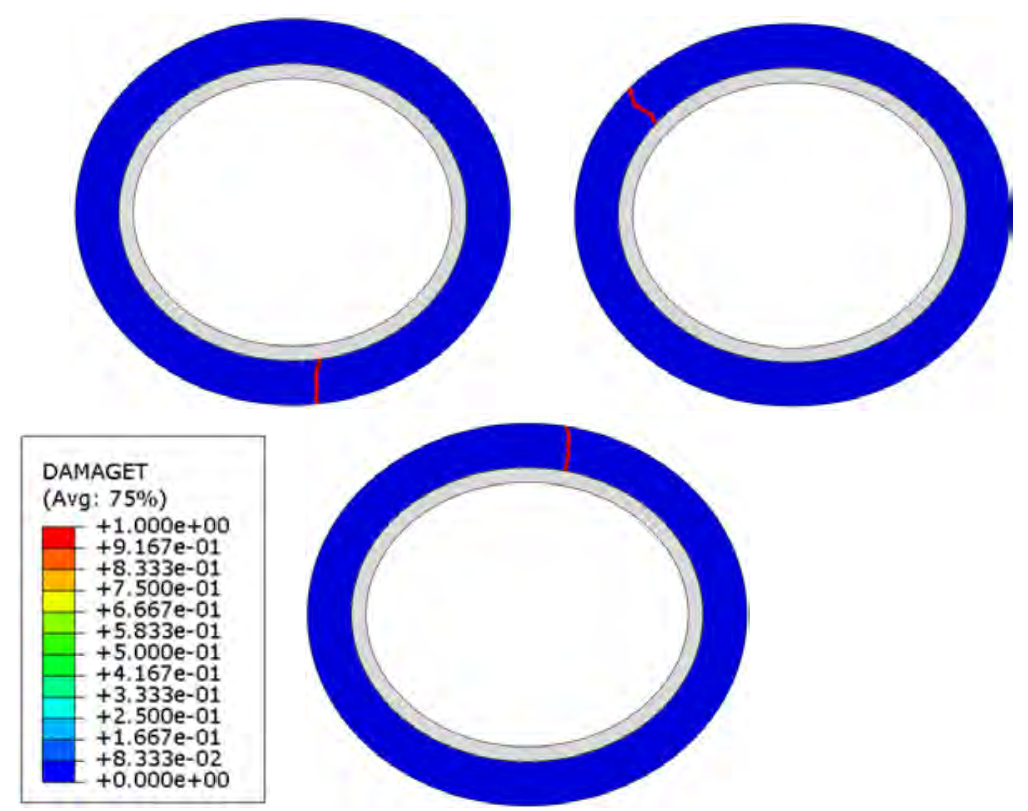

Figura 5.12: Zonas dañadas para simulaciones realizadas con mallado triangular y material aleatorio para el anillo 2

Finalmente, la Figura 5.13 muestra las fisuras obtenidas experimentalmente para el anillo elíptico 3. Las Figuras 5.14 y 5.15, muestran las zonas 
dañadas correspondientes a los modelos con distribución aleatoria de las propiedades mecánicas, con mallado rectangular y triangular, respectivamente.

Los resultados experimentales muestran que las fisuras aparecen cercanas al radio mayor, lo cual se verifica en los resultados obtenidos con ambos tipos de elementos y distribución aleatoria de las propiedades mecánicas.
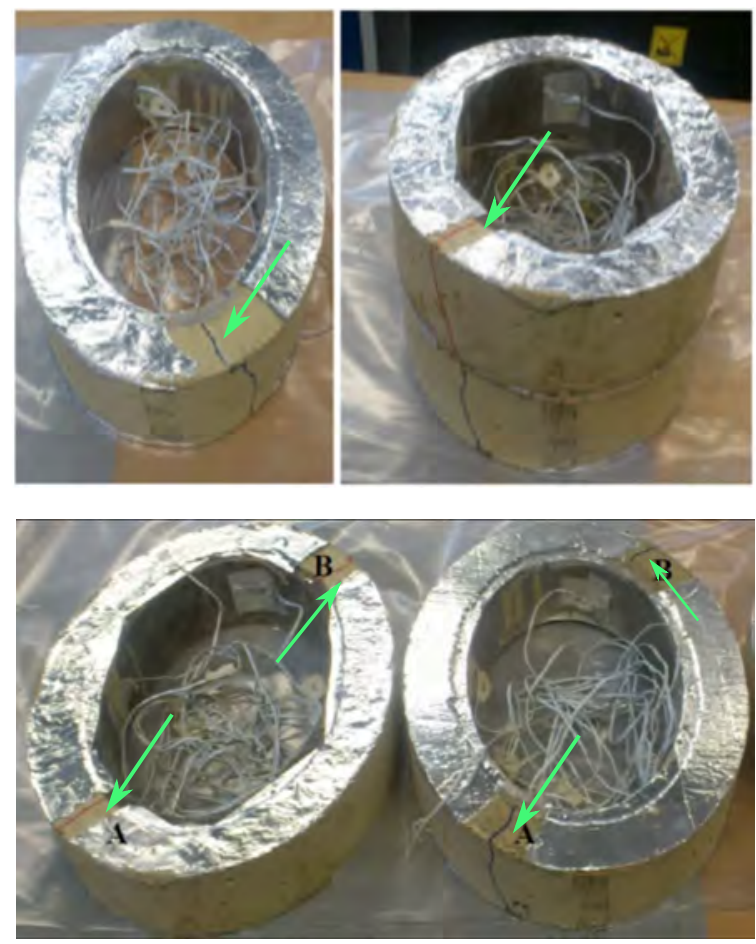

Figura 5.13: Ubicación de las fisuras en ensayos experimentales para el anillo 3, Dong et al. (2016) 
5.3. Validación del algoritmo desarrollado a partir de los resultados de otros autores

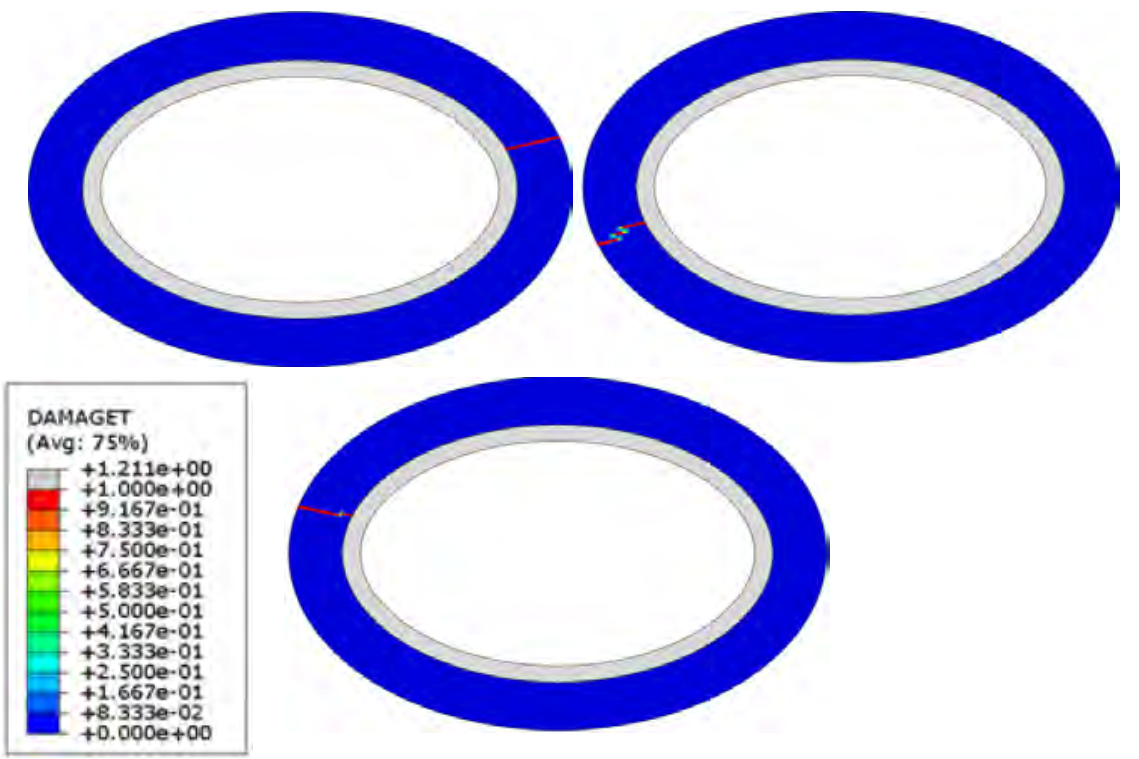

Figura 5.14: Zonas dañadas para las simulaciones realizadas con mallado cuadrado y material aleatorio para el anillo 3

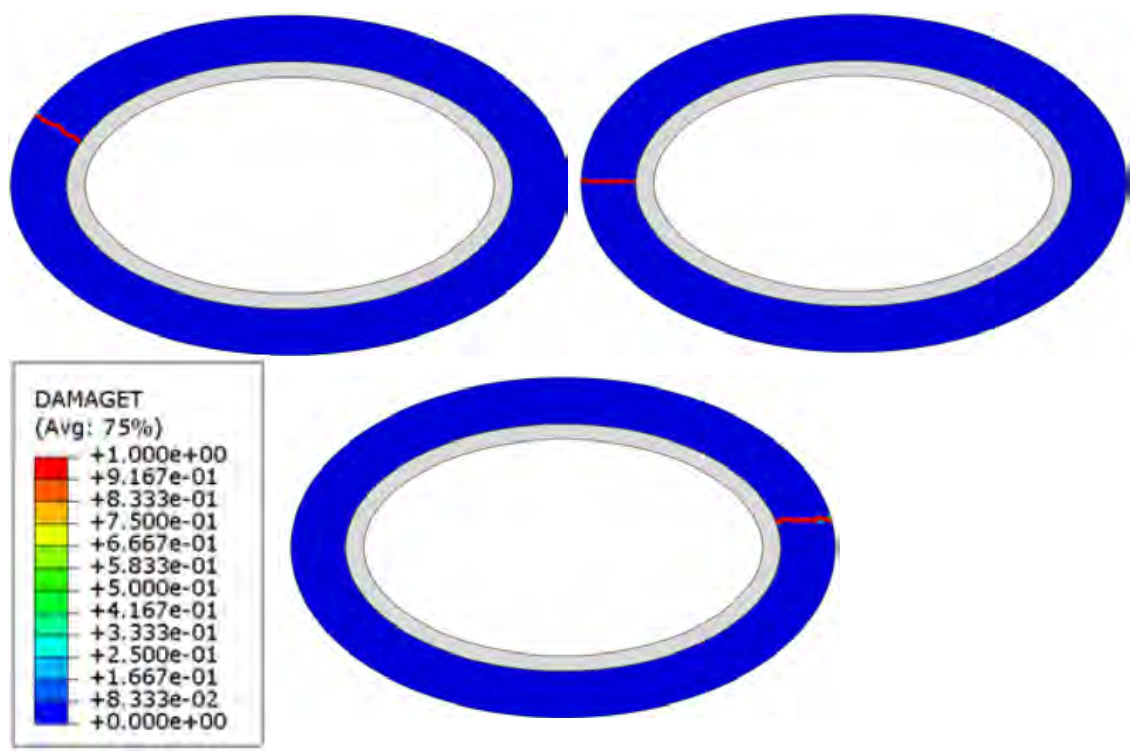

Figura 5.15: Zonas dañadas para las simulaciones realizadas con mallado triangular y material aleatorio para el anillo 3 



\section{Capítulo 6}

\section{Conclusiones y Trabajo Futuro}

En el último capítulo de esta Tesis Doctoral se resumen las tareas realizadas, se presentan las principales conclusiones y se proponen algunas posibles líneas de trabajo futuro.

\subsection{Conclusiones}

- Se desarrolló un criterio basado en la heterogeneidad del material que, asociado a un Modelo de Plasticidad con Daño, permite definir zonas de fisuración preferentes en estructuras de hormigón sometidas a estados tensionales macroscópicamente uniformes, como es el caso de la contracción por secado en el ensayo de contracción restringida en anillos de hormigón.

- Para la implementación del criterio propuesto se desarrolló un algoritmo en el entorno MatLab, que permite asignar en forma aleatoria las propiedades mecánicas al hormigón, considerando que dichas propiedades varían según una función de distribución de probabilidad, como por ejemplo una función de Gauss o una función de Weibull.

- El algoritmo desarrollado considera la evolución temporal de las propiedades mecánicas del hormigón, lo cual constituye un aspecto de fundamental importancia para el estudio de fisuración a edad temprana, como es el caso de la contracción por secado, ya que tanto la resistencia a tracción y compresión, como el módulo de elasticidad, se incrementan de manera significativa hasta la edad de 28 días.

- La selección del modelo para la implementación del algoritmo surgió a partir de la comparación entre el Método de los Elementos Finitos Extendido (XFEM), el Modelo de Fisuración Cohesiva (MFC) y un Modelo de Plasticidad con Daño (MPD). El primero de ellos resultó no ser el adecuado ya que solamente permite que en cada enriquecimiento 
se genere una sola fisura, o más de una siempre y cuando todas ocurran de manera simultánea, y en los casos analizados solo es posible definir un único enriquecimiento. En cuanto al MFC, su implementación es considerablemente trabajosa ya que para la resolución de los problemas abordados en esta Tesis es necesario incorporar elementos cohesivos en todas las interfases entre elementos convencionales lo que origina un incremento considerable en el tiempo de cálculo y además introduce un aumento de la flexibilidad a la geometría que no se condice con la realidad física del problema en estudio. Finalmente, la elección del MPD se debe a que si bien es un modelo continuo que permite definir zonas dañadas y no el camino de una fisura, su implementación puede llevarse a cabo con mayor generalidad, al ser aplicado directamente a las propiedades mecánicas del material en toda la geometría analizada.

- Para la validación del funcionamiento del algoritmo se simuló numéricamente el ensayo de contracción restringida en anillos de hormigón circulares, con y sin entalla, y elípticos sin entallar. Los resultados de las simulaciones se compararon con ensayos de laboratorio propios realizados en anillos circulares y con ensayos disponibles en la bibliografía correspondientes a anillos elípticos. En todos los casos, los resultados numéricos obtenidos aplicando el algoritmo desarrollado, tanto aquellos correspondientes a la determinación de la edad de fisuración como a la ubicación de las zonas fisuradas, han sido coincidentes con los resultados experimentales.

- Se desarrolló un procedimiento experimental mediante el cual fue posible obtener un factor variable en el tiempo que permite cuantificar el efecto del creep en el anillo de hormigón durante el ensayo de contracción restringida. Se aplicó el método del módulo de elasticidad efectivo considerando un factor de reducción variable en el tiempo aplicado al módulo de elasticidad instantáneo, y no un factor de reducción constante como se utiliza en la bibliografía, observándose una mejora en la predicción numérica.

- El criterio propuesto fue utilizado con éxito para simular numéricamente un ensayo de tracción directa sobre una probeta de hormigón simple sin entallas. Los resultados obtenidos aplicando el algoritmo de asignación aleatoria de propiedades y el Modelo de Plasticidad con Daño se corresponden con el comportamiento experimental observado, siendo posible además recuperar el comportamiento cuasifrágil característico del hormigón en la curva carga-desplazamiento.

- Analizando geometrías con la misma relación de aspecto sometidas a contracción por gradientes de temperatura, aplicando el algoritmo desarrollado y un Modelo de Plasticidad con Daño, se obtienen zonas 
dañadas independientes de las condiciones de contorno mecánicas, lo cual no ocurre al utilizar propiedades uniformes. Estas zonas representan fisuras de superficie.

- El algoritmo presentado se desarrolló en base a distribuciones probabilísticas de las propiedades mecánicas del material. Esto le otorga rigor al estudio permitiendo hacer muchas simulaciones para un mismo problema, obteniéndose en cada caso resultados diferentes, y un posterior análisis estadístico de los resultandos lo cual le otorga un enfoque más realista al análisis de los problemas abordados.

- Al comparar los resultados experimentales propios y los disponibles en la bibliográfía con los resultados numéricos obtenidos, puede concluirse que la validación del algoritmo desarrollado aplicado junto con un Modelo de Plasticidad con Daño para el estudio del proceso de fisuración por contracción fue exitosa.

\subsection{Trabajo futuro}

Entre las principales líneas de trabajo futuro derivados de la presente Tesis Doctoral se proponen las siguientes:

- Extender la aplicación del algoritmo desarrollado a tres dimensiones, ya que en algunos problemas, como por ejemplo el estudio de la fisuración por contracción térmica en el módulo de una presa de hormigón, no es correcto realizar análisis tensionales en dos dimensiones. En consecuencia, para poder abordar su estudio, es necesario disponer de modelos tridimensionales.

- Profundizar en el estudio de la determinación de un coeficiente de creep para aplicar a la modelación numérica de la fisuración a edad temprana en hormigón debido a contracción restringida, mediante la realización de ensayos de creep en tracción y flexión a carga variable. En este último caso se contempla el desarrollo del dispositivo experimental para la ejecución del ensayo.

- Extender el algoritmo desarrollado, considerando la presencia de fibras de refuerzo en el hormigón. Esto se considera de gran interés dado que el control de fisuración a edad temprana es una de las razones de la incorporación de este tipo de fibras de refuerzo. Se contempla la realización de una extensa campaña experimental, utilizando fibras de distinto tipo y tamaño, y la comparación de los efectos de cada una de ellas en los resultados obtenidos.

- Por último, se propone incorporar el efecto de los gradientes de humedad al algoritmo para estudiar la contracción por secado en elementos 
de gran espesor, ya que en estos casos no es válida la suposición de que la variación de la humedad es uniforme en todo el espesor del elemento. 


\section{Bibliografía}

ABAQUS. ABAQUS 6.13 Analysis User's Manual Volume II: Analysis. Dassault Systèms Simulia Corp., 2013a.

ABAQUS. ABAQUS 6.13 Analysis User's Manual Volume III: Materials. Dassault Systèms Simulia Corp., 2013b.

ABAQUS. ABAQUS 6.13 Analysis User's Manual Volume IV: Elements. Dassault Systèms Simulia Corp., 2013c.

ABAQUS. ABAQUS 6.13 Analysis User's Manual Volume V: Prescribed conditions, constraints and interactions. Dassault Systèms Simulia Corp., 2013d.

ABAQUS. ABAQUS 6.13 Theory manual. Dassault Systèms Simulia Corp., 2013e.

ACI. ACI 207.2R-07 Report on Thermal and Volume Change Effects on Cracking of Mass Concrete. American concrete institude, 2007.

ACI. ACI 214R-11: Guide to evaluation of strength test results of concrete. ACI committee 214, 2011.

ACI. ACI 318S-14: Building code requirementes for strcutural concrete. ACI committee 318, 2014.

Aly, T. y Sanjayan, J. G. Mechanism of early age shrinkage of concretes. Materials and Structures, vol. 42, páginas 461-468, 2009.

Areias, P. M. A. y Belytschko, T. A comment on the article: A finite element method for the simulation of strong and weak discontinuities in solid mechanics. Computer methods in applied mechanics and engineering, vol. 195, páginas 1275-1276, 2005.

Arregui-Mena, J. D., Margetts, L. y Mummery, P. M. Practical application of the stochastic finite element method. Archives of computational methods in engineering, vol. 23(1), páginas 171-190, 2016. 
ASTM. C 1581/C 1581M-09 standard test method for determinng age at cracking and induced tensile stress characteristics of mortar and concrete under restrained shrinkage. ASTM International, vol. -, páginas 1-7, 2004a.

ASTM. C 39-04a Compressive strength of cylindrical concrete specimens. ASTM International, vol. -, páginas -, 2004b.

ASTM. C 496/C 496M-04 Standard test method for splitting tensile strength of ciylindrical concrete specimens. ASTM International, vol. -, páginas 15, 2004c.

ASTM. C341/C341M-13 standard practice for preparation and conditioning of cast, drilled, or sawed specimens of hydraulic-cement mortar and concrete used for length change measurements. ASTM International, vol. -, páginas 1-4, 2013a.

ASTM. E1875-08 Standard test method for dynamic Yung's modulus, shear modulus, and Poisson's ratio by sonic resonance. ASTM International, vol. -, páginas 1-9, 2013b.

ASTM-C-469-02. C 469-02 Standard test method for static modulus of elasticity and poisson's ratio of concrete in compression. ASTM International, vol. -, páginas 1-5, 2002.

Attiogbe, E. K., See, H. T. y Miltenberger, M. A. Cracking potential of concrete under restrained shrinkage. Proc. Advances in Cement and Concrete: Volume Changes. Cracking and Durability. Engineering Conferences International, Copper Mountain, CO, vol. -, páginas 191-200, 2003.

Au, S.-K. y BECK, J. Estimation of small failure probabilities in high dimensions by subset simulation. Probabilistic Engineering Mechanics, vol. 4, páginas 263-277, 2001.

BA, H., Su, A., GaO, X. y TAO, Q. Cracking tendency of restrained concrete at early ages. Journal Wuhan University Technology Material Science, vol. 23, páginas 263-267, 2008.

BAREnBLATT, G. I. The mathematical theory of equilibrium cracks in brittle fracture. Advanced applied mechanics, vol. 7, páginas 55-129, 1962.

Bażant, Z., Cusatis, G. y Cedolin, L. Temperature effect on concrete creep modeled by microprestress-solidification theory. Journal of Engenieering Mechanics, vol. 130, páginas 691-699, 2004.

Baẑant, Z., Hauggaard, A., Baweja, S. y Ulm, F.-J. Microprestresssolidification theory for concrete creep I: Aging and drying effects. Journal of Engenieering Mechanics, vol. 123, páginas 1188-1194, 1997. 
BAẐAnt, Z. y PANG, S. Activation energy based extreme value statistics and size effect in brittle and quasibritlle fracture. Journal of mechanics and physiscs of solids, vol. 55 (1), páginas 91-131, 2007.

Baẑant, Z., Pang, S., Vorechovsky, M. y Novák, D. Energeticstatistical size effect simulated by sfem with stratified sampling and crack band model. International journal for numerical method in engineering, vol. 71, páginas 1297-1320, 2007.

BAẐAnT, Z. y XI, Y. Drying creep of concrete: Constitutive model and new experiments separating its mechanisms. Materials and structures, vol. 27(1), páginas 3-14, 1994.

BAẐAnt, Z. P. y Li, Y. N. Stability of cohesive crack model: Part II eigenvalue analysis of size effect on strength and ductility of structures. Journal of applied mechanics T. ASME, vol. 62, páginas 965-969, 1995.

BaẑAnt, Z. P. y NovaK, D. Energetic-statistical size effect in quasibrittle failure at crack initiation. ACI Material journal, vol. 97(3), páginas 381392, 2000a.

BaẑAnt, Z. P. y NovaK, D. Probabilistic nonlocal theory for quasibrittle fracture initiaton and size effect II: aplication. Journal of engineering mechanics, vol. 126 (2), páginas 175-185, 2000b.

BaẐAnt, Z. P. y Planas, J. Fracture and size effects in concrete and other quasibritle materials. CRC Press, 1997.

BaẑAnt, Z. P., XI, Y. y ReID, S. G. Statistical size effect in quasi-brittle structures, I. Is weibull theory applicable? Journal of engineering mechanics, vol. 117 (11), páginas 2609-2622, 1991.

Belytschko, T. y Black, T. Elastic crack growth in finite elements with minimal remeshing. International journal for numerical method in engineering, vol. 45, páginas 601-620, 1999.

Benaroya, H. y RehaK, M. Finite element methods in probabilistic structural analysis: a selective review. Applied Mechanics Review, vol. 41, páginas 201-213, 1988.

Bentur, A. Early age cracking in cementitous systems: Report of RILEM technical comitee 181-EAS: "Early age shrinkage induced stressess and cracking in cementitious systems". RILEM, vol. -, páginas -, 2003.

Biffaut, M., Benboudjema, F., Torrenti, J. M. y Nahas, G. Numerical analysis of the active restrained shrinkage ring test to study the early age behavior of massive concrete structures. Engineering Structures, vol. 33, páginas 1390-1401, 2011.

Ing. M. Paula Zappitelli 
Bouhjitia, D.-M., Barotha, J., Briffauta, M., Dufoura, F. y MasSOND, B. Statistical modeling of cracking in large concrete structures under thermo-hydro-mechanical loads: Application to nuclear containment buildings. Part 1: Random field effects (reference analysis). Nuclear Engineering and Design, vol. 333, páginas 196-223, 2018.

Brooks, J. J. Concrete and Masonry Movements. Elsevier, 2015.

Cannas, S. Notas de Mecánica estadística. Editorial de la UNC, 2018.

Carlson, R. S. y Reading, T. J. Model of studing shrinkage cracking in concrete building wall. ACI Structures Journal, vol. 85(4), páginas 395-404, 1998.

Carol, I., Rizzi, E. y Willam, K. On the formulation of anisotropic elastic degradation I: Theory based on a pseudo-logarithmic damage tensor rate. International Journal of Solids and Structures, vol. 38, páginas 491-518, 2001.

Carpinteri, A., Ferro, G. y Monetto, I. Scale effects in uniaxially compress concrete specimens. Magazzine of concrete research, vol. 51(3), páginas 217-225, 1999.

CAVAzZuti, M. Optimization methods: from theory to design scientific and technological aspects in mechanics. 2012.

CEB-FIB. CEB-FIP Model code 1990: Desing code. -, 1993.

Chaudhar, S. y Chakrabart, M. Modeling of concrete for nonlinear analysis using finite element code ABAQUS. International Journal of Computer Applications, vol. 4(7), páginas 14-18, 2012.

Chen, H., Xu, B., Mod, Y. L. y Zhou, T. Behavior of meso-scale heterogeneous concrete under uniaxial tensile and compressive loadings. Construction and Building Materials, vol. 178, páginas 418-431, 2018.

CIRIA-660. Early-age thermal crack control in concrete. CIRIA, 2007.

Copeland, L. E. y BRAgG, R. H. Self desiccation in portland cement pastes. Research Department Bulletin RX052, Portland Cement Association, vol. -, páginas -, 1955 .

C, J. y Papanikolaou, V. Three dimensional combined fracture-plastic material model for concrete. International Journal of Plasticity, vol. 24, páginas 2192-2220, 2008.

Der Kiureghian, A. y Ke, J.-B. The stochastic finite element method in structural reliability. Probabilistic Engineering Mechanics, vol. 3(2), páginas 83-91, 1988. 
Dong, W., Yuan, W. y Zhou, F., X. Wang. The fracture mechanism of circular/elliptical concrete rings under restrained shrinkage and drying from top and bottom surfaces. Engineering Fracture Mechanics, vol. -, páginas -, 2017.

Dong, W., Zhou, X. y Wu, Z. A fracture mechanics-based method for prediction of cracking of circular and elliptical concrete rings under restrained shrinkage. Engineering Fracture Mechanics, vol. 131, páginas 687-701, 2014 .

Dong, W., Zhou, X., Wu, Z. y Kastiukas, G. Effects of specimen size of shrinkage cracking of concrete via elliptical rings: Thin vs. thick. Computer and Structures, vol. 174, páginas 66-78, 2016.

Dong, W., Zhou, X. M. y Wu, Z. M. Influence of specimen thickness on cracking behavior in restrained shrinkage ring test. IACSIT International Journal of Engineering and technology, vol. 5(6), páginas 698-702, 2013.

Drucker, D. C. y Prager, W. Soil mechanics and plastic analysis for limit design. Quartely of Applied Mathematics, vol. 10, páginas 157-165, 1952.

DU, X., JIN, L. y MA, G. Meso-element equivalent method for the simulation of macro mechanical properties of concrete. International journal of damage mechanics, vol. 22, páginas 617-642, 2012.

Duarte, C. y Oden, J. An hp adaptive method using clouds. Computer Method in Applied Mechanics and Engineering, vol. 139, páginas 237-262, 1996.

Dugdale, D. S. Yielding of steel sheets containing slits. Journal of mechanics and physics of solids, vol. 8, páginas 100-108, 1960.

Elías, J., Vorechovskyý, M., Skocek, J. y Baẑant, Z. P. Stochastic discrete meso-scale simulations of concrete fracture: Comparison to experimental data. Engineering Fracture Mechanics, vol. 135, páginas 1-16, 2015 .

Elices, M. y Planas, J. Material models in Fracture mechanics of concrete structures. Elfgren ed. Chapman and Hall, 1989.

Elices Calafat, M. Mecánica de la fractura aplicada a sólidos bidimensionales. Departamento de ciencia de materiales. Escuela técnica superior de ingenieros de caminos canales y puertos. Universidad Politécnica de madrid, 1998.

EsPing, O. Early age properties of self-compacting concrete. Effects of fine aggregate and limestone filler. Ph.D. Thesis, Chalmers University of Technology, Sweden, 2007.

Ing. M. Paula Zappitelli 
Etse, G. y Willam, K. A fracture-energy based constitutive formulation for inelastic behavior of plain concrete. Journal of Engineering Mechanics, $A S C E$, vol. 120, páginas 1983-2011, 1994.

Evan, R. H. y Marathe, M. S. Microcracking and stress-strain curves for concrete in tension. Material and Structures, vol. 1, páginas 61-64, 1968.

Flaga, K. y Furtak, K. Problem of thermal and shrinkage cracking in tanks vertical walls and retaining walls near their contact with solid foundation slabs. Architecture civil engineering environment, vol. 2, páginas 23-30, 2009.

Folino, P. y Etse, G. Performance dependent model for normal and high strength concretes. International Journal of Solids and Structures, vol. 49, páginas 701-719, 2012.

Gentle, J. E. Random Number Generator and Monte Carlo Methods. Springer Science \& Business Media, 2004.

Ghanem, R. y Spanos, P. Stochastic finite elements: a spectral approach. 2003.

Giovambattista, A., Violini, D. y Zerbino, R. Extensibilidad de hormigones con cenizas volantes. Asociación Argentina de Tecnología del Hormigón, vol. 8, páginas 359-374, 1987.

Giovambattista, A., Violini, D. y Zerbino, R. Extensibility of highstrength concretes. Materials and structures, vol. 25, páginas 552-557, 1992.

Grassl, P. On a damage-plasticity approach to model concrete failure. Proceedings of the ICE Engineering and Computational Mechanics, vol. 162, páginas 221-231, 2009.

Grassl, P. y JirÁseK, M. Damage-plastic model for concrete failure. International Journal of Solids and Structures, vol. 43, páginas 7166-7196, 2006.

Grassl, P., Lundgren, K. y Gylltoft, K. Concrete in compression: a plasticity theory with a novel hardening law. International Journal of Solids and Structures, vol. 39, páginas 5205-5223, 2002.

Grassl, P., Xenos, X., Nystr"om, U., Rempling, R. y Gylltoft, K. CDPM2: A damage-plasticity approach to modelling the failure of concrete. International Journal of Solids and Structures, vol. 50, páginas 3805-3816, 2013.

Griffith, A. A. The phenomena of rupture and flow in solids. Philos Trans Roy Soc A, vol. 221, páginas 163-197, 1921. 
Griffith, A. A. The theory of rupture. Proceedings of the first international conference of applied mechanics, vol. -, páginas 55-63, 1924.

Grzybowski, M. y Shah, S. P. Shrinkage cracking of fiber reinforced concrete. ACI Material Journal, vol. 87 (2), páginas 138-148, 1990.

Hansbo, A. y Hansbo, P. A. A finite element method for the simulation of strong and weak discontinuities in solid mechanics. Computer methods in applied mechanics and engineering, vol. 193, páginas 3523-3540, 2004.

HARBitz, A. An efficient sampling method for probability of failure calculation. Structural safety, vol. 3, páginas 109-115, 1986.

Hariri-Ardebili, M., Seyed-Kolbadi, S., Saouma, V., Salamon, J. y Rajagopalan, B. Random finite element method for the seismic analysis of gravity dams. Engineering Structures, vol. 171, páginas 405-420, 2018.

VAN DER HAVE, R. Random fields for non-linear finite element analysis of reinforced concrete. M.S. Thesis Delft University of Technology, Netherlands, 2015.

Hillerborg, A., Modéer, M. y Petersson, P. E. Analysis of crack formation and crack growth in concrete by means of fracture mechanics and finite elements. Cement concrete res., vol. 6, páginas 773-782, 1976.

Holt, E. Contribution of mixture design to chemical and autogeneous shrinkage of concrete at early ages. Cement and Concrete Research, vol. 35 (3), páginas 464-472, 2005.

Holt, E. E. Early age autogenous shrinkage of concrete. VTT, 2001.

Hossain, A. B. y Weiss, J. The role of specimen geometry and boundary conditions on stress development and cracking in the restrained ring test. Cement and Concrete Research, vol. 36, páginas 189-199, 2006.

Houghton, D. L. J. ACI proceedings. ACI, vol. 73(12), páginas 691-700, 1976.

Huang, Y., Yan, D., YAng, Z. y LiU, G. 2D and 3D homogenization and fracture analysis of concrete based on in-situ x-ray computed tomography images and Monte Carlo simulations. Engineering Fracture Mechanics, vol. 163, páginas 37-54, 2016.

Hughes, B. P. y Chapman, G. P. The complete stress-strain for concrete in direct tension. RILEM Bulletin, vol. 30, páginas 95-97, 1966.

IngLis, C. E. Stresses in a plate due to the presence of cracks and sharp corners. Trans Inst Naval Archit, vol. 55, páginas 219-241, 1913.

Ing. M. Paula Zappitelli 
Ingraffea, A. R. Encyclopedia of Computacional Mechanics. John Wiley and Sons, Ltd., 2007.

IRAM-1524. Preparación y curado en obra de probetas para ensayos de compresión y de tracción por compresión diametral. Argentina, 1982.

IRAM-1622. Hormigones y morteros. Determinación del tiempo de fraguado. -, vol. -, páginas 1-12, 1995.

IRWING, G. R. Analysis of stresses and strains near the end of a crack traversing a plate. Journal of Applied Mech Trans ASME, vol. 24, páginas 361-364, 1957.

Jason, L., Huerta, A., Pijaudier-Cabot, G. y Ghavamian, S. An elastic plastic damage formulation for concrete: application to elementary tests and comparison with an isotropic damage model. Computer Methods in Applied Mechanics and Engineering, vol. 195, páginas 7077-7092, 2006.

JEHEL, P. A stochastic multi-scale approach for numerical modeling of complex materials-Application to uniaxial cyclic response of concrete. Computational Methods for Solids and Fluids, vol. 41, páginas 123-160, 2016.

Ju, J. On energy-based coupled elastoplastic damage theories: constitutive modeling and computational aspects. International Journal of Solids and Structures, vol. 25, páginas 803-833, 1989.

Kachanov, M. Continuum model of medium with cracks. Journal of the Engineering Mechanics Division, vol. 106, páginas 1039-1051, 1980.

Khairallah, R. Analysis of autogenous and drying shrinkage of concrete. McMaster University, 2009.

KlemcZaK, B. y KnoppiK-WróBel, A. Early age thermal and shrinkage cracks in concrete structures- influence of curing conditions. Architecture civil engineering environment, vol. 4, páginas 47-58, 2011.

Knuth, D. E. Seminumerical Algorithms, The art of computer programming. Pearson Education, 1997.

Kosmatka, S. H., Kerkhoff, B. y Panarese, W. C. Design and Control of Concrete Mixtures. Portland Cement Association, 2002.

Kovler, K. Testing system for determining the mechanical behavior of early age concrete under restrained and free uniaxial shrinkage. Rilem Material Structures, vol. 27 (6), páginas 324-330, 1994.

KraAi, P. P. A proposed test to determine the craking potential due to drying shrinkage of concrete. Concrete Construction, vol. 30 (9), páginas $775-778,1985$. 
Kucharczyková, B., Danjk, P., Kocáb, D. y Misák, P. Experimental analysis on shrinkage and swelling in ordinary concrete. Advances in Materials Science and Engineering, vol. -, páginas 1-11, 2017.

Kumar, S. y Barai, S. V. Concrete Fracture Models and Applications. Springer, 2011.

KWAna, A., NG, P. y Wang, Z. Mesoscopic analysis of crack propagation in concrete by nonlinear finite element method with crack queuing algorithm. Procedia engineering, vol. 172, páginas 620-627, 2017.

L' Hermite, R. Volume changes of concrete. Proceedings of 4 th international symposium on the chemistry of cement, Washington DC, vol. -, páginas 659-694, 1960.

Le, J. L., Eliâŝ, J. y Bâ̂Ant, Z. P. Computation of probability distribution of strength of quasibrittle structures failing at macrocrack initiation. Journal of engineering mechanics, vol. 138, páginas 888-899, 2012.

Lee, J. y Fenves, G. L. Plastic-damage model for cyclic loading of concrete structures. Engineering Mechanics, vol. 124, páginas 892-900, 1998.

López, C., Carol, I. y Aguado, A. Meso-structural study of concrete fracture using interface elements. II: Compression, biaxial and Brazilian test. Material and structures, vol. 41(3), páginas 601-620, 2007.

Lubliner, J., Oliver, J., Oller, S. y Oñate, E. A plastic-damage model for concrete. Solid Structures, vol. 25, páginas 299-236, 1989.

Lura, P., Jensen, O. M. y Van, B. K. Autogenous shrinkage in highshrinkage cement paste: An evaluation of basic mechanisms. Cement and Concrete Research, vol. 33(2), páginas 223-232, 2003.

Mahadevan, S. y Haldar, A. Practical random field discretization in stochastic finite element analysis. Structural Safety, vol. 9, páginas 283304, 1991.

Matthies, H. G., Brenner, C. E., Bucher, C. G. y Sohres, C. G. Uncertainties in probabilistic numerical analysis of structures and solidsStochastic finite elements. Structural Safety, vol. 19(3), páginas 283-336, 1997.

Mazars, J. y Pijaudier-CABot, G. Continuum damage theoryapplication to concrete. Journal of Engineering Mechanics, vol. 115, página 345, 1989.

McKay, M., Beckma, R. y Conover, W. A comparison of three methods for selecting values of input variables in the analysis of output from a computer code. Technometrics, vol. 21, páginas 239-245, 1979. 
Melenk, J. y BABÛskA, I. The partition of unity finite element method: Basic theory and applications. Computer method in applied mechanics and engineering, vol. 139, páginas 289-314, 1996.

Menétrey, P. y Willam, K. A triaxial failure criterion for concrete and its generalization. ACI Structural Journal, vol. 92, páginas 311-318, 1995.

Metha, P. K. y Monteiro, P. J. M. Concreto, estructura, propiedades y materiales. Instituto mexicano del cemento y del concreto, A. C., 1998.

VAN Mier, J. G. M. Fracture processes of concrete. CRC Press, 1997.

Mindess, S., Young, J. F. y Darwin, D. Concrete. Prentice Hall, 2003.

MoËs, N., Dolbow, J. y BelyschKo, T. A finite element method for crack growth without remeshing. International Journal for numerical methods in engineering, vol. 46, páginas 131-150, 1999.

Moon, J. H., Rajabipour, F., Pease, B. y Weiss, J. Quantifying the influence of the restrained ring test. Journal of ASTM International, vol. 3 (5), páginas $1-13,2006$.

Moreno, R. S., Rojano Aguilar, A., Figueroa Hernández, E. y Pérez Soto, F. Aplicaciones de la distribución weibull en ingeniería de confiabiliad. Memoria del XXI Coloquio Mexicano de Economía Matemática y Econometría, vol. -, páginas 148-161, 2011.

Neville, A. M. Properties of Concrete. Pearson, 2011.

Newman, J. y Choo, B. Advanced Concrete Technology. Constituent Materials. Elsevier, 2003.

Nguyen, G. y Houlsby, G. A coupled damage-plasticity model for concrete based on thermodynamic principles. Part I: Model formulation and parameter identification. International Journal for Numerical and Analytical Methods in Geomechanics, vol. 32, páginas 353-389, 2008.

NGuyen, G. y Korsunsky, A. Development of an approach to constitutive modelling of concrete: isotropic damage coupled with plasticity. International Journal of Solids and Structures, vol. 45, páginas 5483-5501, 2008.

Omidi, O., y LotFi, V. Finite element analysis of concrete structures using plastic-damage model in 3-D implementation. International Journal of Civil Engineerng, vol. 8 (3), páginas 187-203, 2010.

OrTIZ, M. A constitutive theory for the inelastic behavior of concrete. Mechanics of Materials, vol. 4, páginas 67-93, 1985. 
Papanikolaou, V. y Kappos, A. Confinement-sensitive plasticity constitutive model for concrete in triaxial compression. International Journal of Solids and Structures, vol. 44, páginas 7021-7048, 2007.

Parilee, A. M., Buil, M. y Serrano, J. J. Effect of fiber addition on the autogenous shrinkage of silica fume concrete. ACI Material Journal, vol. 86 (2), páginas 139-144, 1989.

Petersson, P. E. Crack growth and development of fracture zone in plane concrete and similar materials. Report No TVBM-1006, Division of building materials, Lund Institude of Technology, Lund Suiza, vol. -, páginas $-, 1981$.

Pickett, G. Effect of change in moisture-content on the creep of concrete under a sustained load. Journal Proceedings, vol. 38, páginas 333-356, 1942.

Pivonka, P. Constitutive modeling of triaxially loaded concrete considering large compressive stresses: application to pull-out tests of anchor bolts Ph.D. thesis.. Technische Universitat Wien, Austria, 2001a.

Pivonka, P. Nonlocal plasticity models for localized failure. Ph.D. Thesis. Technische Universitat Wien, Austria., 2001b.

Planas, J. y Elices, M. Shrikage eigenstresses and structural size-effect in Fracture Mechanics of concrete structures, Z. P. Bażant. Elservier applied science, 1992.

Planas, J., Elices, M. y Guinea, G. V. The extended cohesive crack in Fractute of brittle disordered materials: Concrete rock and ceramic. G. Bakker and B. L. Karihaloo eds, E \& FN Spon, 1995a.

Planas, J., Guinea, G. V. y Elices, M. Rupture modulus and fracture properties of concrete in Fracture mechanics of concrete structures, Vol I F. H. Wittmann. Aedificatio Publishers, 1995b.

Planas, J., Guinea, G. V. y Elices, M. Generalized size effect equation for quasibrittle materials. Fatigue Fracture in engineering material structures, vol. 20, páginas 671-687, 1997.

Plizzari, G. A. y Saouma, V. E. Linear or nonlinear fracture mechanics of concrete? Proceedings of 2nd International conference on fracture mechanics of concrete structures, FRAMCOS2, F.H. Wittman, ed., Aedificato, Freiburg, Germany, vol. -, páginas 1377-1386, 1995.

Power, T. C. Absorption of water by portland cement paste during the hardening process. Industrial and Engineering Chemistry, vol. 27(7), páginas,- 1935.

Ing. M. Paula Zappitelli 
Pramono, E. y Willam, K. Fracture energy-based plasticity formulation of plain concrete. Journal of Engineering Mechanics, ASCE, vol. 115, páginas 1183-1203, 1989.

RADOCEA, A. A study on the mechanism of plastic shrinkage of cement-based materials. Doctor Thesis, Chalmers University of Technology, Gothenburg, 1992.

Remmers, J. J. C., De Borst, R. y Needleman, A. The simulation of dynamic crack propagation using the cohesive segment method. Journal of the mechanics and physics of solids, vol. 56, páginas 70-92, 2008.

Resende, L. A damage mechanics constitutive theory for the inelastic behaviour of concrete. Computer Methods in Applied Mechanics and Engineering, vol. 60, páginas 57-93, 1987.

RILEM. Determination of the fracture energy of mortar and concrete by means of three-point bend tests on notched beams. Materials and structures, vol. 18(106), páginas -, 1985.

Sánchez, P., Huespe, A., Oliver, J., Diaz, G. y Sonzogni, V. A macroscopic damage-plastic constitutive law for modeling quasi-brittle fracture and ductile behavior of concrete. International Journal for Numerical and Analytical Methods in Geomechanics, vol. 36, páginas 546-573, 2011.

SAYAHI, F. Plastic shrinkage cracking in concrete. Licentiate Thesis, Lulea University of Technology, 2016.

Schmidt, M. y Slowik, V. Capillary pressure controlled concrete curing in pavement construction. Proceedings of the 2013 Airfield and Highway Pavement Conference, vol. -, páginas 295-306, 2013.

Sencu, R., YAng, Z. y Y.C., W. An adaptive stochastic multi-scale method for cohesive fracture modelling of quasi-brittle heterogeneous materials under uniaxial tension. Engineering Farcture Mechanics, vol. 163, páginas 499-522, 2016.

Shales, C. A. y Hover, K. C. Influence of mix-proportion and construction operations on plastic shrinkage cracking in thin slabs. ACI Material Journal, vol. 85 (6), páginas 495-504, 1988.

Song, J. H., Areias, P. M. y Belytschko, T. A method for dynamic crack and shear band propagation with phantom nodes. International journal for numerical method in engineering, vol. 67, páginas 868-893, 2006.

Stefanou, G. The stochastic finite element method: Past, present and future. Computer methods in applied mechanics and engineering, vol. 198, páginas 1031-1051, 2009. 
Stefanou, G., Georgioudakis, M. y Papadrakakis, M. Sequentially linear analysis of structures with stochastic material properties. Multiscale Modeling and Uncertainty Quantification of Materials and Structures, vol. -, páginas 19-32, 2014.

Su, X. T., Yang, Z. J. y Liu, G. H. Monte Carlo simulation of complex cohesive fracture in random heterogeneous quasi-brittle materials: A 3D study. International journal of solid and structures, vol. 47, páginas 2336$2345,2010$.

Sudret, B. y Der Kiureghian, A. Stochastic finite element methods and reliability: a state-of-the-art report. University of California Berkele, 2000.

Sudret, B. y Der Kiureghian, A. Comparison of finite element reliability methods. Probabilistic Engeneering Mechanics, vol. 17, páginas 337-348, 2002.

Sukumar, N. y Perévost, J. H. Modeling quasi-static crack growth with the extended finite element method. part I: Computer implementation. International Journal of Solid and Structures, vol. 40, páginas 7513-7537, 2003.

Tailhan, J.-L., Rossi, P. y Caucci, A. M. Probabilistic modelling of cracking in concrete structures. European Journal of Environmental and Civil Engineering, vol. 18, páginas 770-779, 2014.

TAL, D. y Fish, J. Stochastic multiscale modeling and simulation framework for concrete. Cement and Concrete Composites, vol. 90, páginas 61-81, 2018.

Tang, S., Yu, Q., Li, H., Yu, C., BaO, C. y Tang, C. Mesomechanical model of moisture diffusion and shrinkage cracking in building materia. Model development. Construction and Building Materials, vol. 47, páginas 511-529, 2013a.

Tang, S. B., Zhang, H., Tang, C. A. y LiU, H. Y. Numerical model for the cracking behavior of heterogeneous brittle solids subjected to thermal shock. International journal of solids and structures, vol. 80, páginas 520$531,2016$.

TAng, X., Y., Z., Zhang, C. y Shi, J. Chapter 23 - study on the heterogeneity of concrete and its failure behavior using the equivalent probabilistic model. Seismic Safety Evaluation of Concrete Dams, vol. -, páginas 541570, 2013b.

TAO, X. y Phillips, D. A simplified isotropic damage model for concrete under bi-axial stress states. Cement and Concrete Composites, vol. 27, páginas 716-726, 2005.

Ing. M. Paula Zappitelli 
Tazawa, E. Autogenous Shrinkage of Concrete. CRC Press, 1999.

Teng, J. G., Zhu, W. y Tang, C. A. Mesomechanical model for concrete. Part II: Applications. Magazine of concrete research, vol. 56(6), páginas 331-345, 2004.

Trawiński, W., Tejcheman, J. y J., B. A three-dimensional meso-scale modelling of concrete fracture, based on cohesive elements and x-ray $\mu \mathrm{CT}$ images. Engineering Fracture Mechanics, vol. 189, páginas 27-50, 2018.

Tumidajski, P. J., Fiore, L., Khodabocus, T., Lachemi, M. y Pari, R. Comparison of Weibull and normal distributions for concrete compressive strengths. Canadian journal of civil engineering, vol. 33, páginas 1287$1292,2006$.

Turon, A., Dávila, C. G., Camanho, P. P. y Costa, J. An engineering solution for mesh size effects in the simulation of delamination using cohesive zone models. Engineering fracture mechanics, vol. 74, páginas 1665-1682, 2007.

Valentini, B. y Hofstetter, B. Review and enhancement of 3D concrete models for large-scale numerical simulations of concrete structures. International Journal for Numerical and Analytical Methods in Geomechanics, vol. 37, páginas 221-246, 2013.

Vanmarcke, E. Random fields: analysis and synthesis. World scientific, vol. -, páginas -, 2010.

Voyiadjis, G. y Kattan, P. A comparative study of damage variables in continuum damage mechanics. International Journal of Damage Mechanics, vol. 18, páginas 315-340, 2009.

Voyiadjis, G., Taqieddin, Z. y Kattan, P. Anisotropic damageplasticity model for concrete. International Journal of Plasticity, vol. 24, páginas 1946-1965, 2008.

Weiss, W. J., y Shah, S. P. Restrained shrinkage cracking: the role of shrinkage reducing admixtures and specimen geometry. RILEM Material Strucures, vol. 35 (3), páginas 85-91, 2002.

WeIss, W. J. Prediction of early-age shrinkage cracking in concrete. Ph.D Disseratation, Northwestern University, Evanston, Illinois, 1999.

Weiss, W. J., Yang, W. y Shah, S. P. Influence of the specimen size/geometry on shrinkage cracking of rings. ASCE Journal of Engineering Mechanics, vol. 126 (1), páginas 93-101, 2000. 
Willam, K. y Warnke, E. Constitutive model for the triaxial behavior of concrete. Concrete Structures Subjected to Triaxial Stresses, IABSE Report, vol. 19, páginas 1-30, 1974.

Yang, Z. J., Su, X. T., Chen, J. F. y Liu, G. H. Monte Carlo simulation of complex cohesive fracture in random heterogeneous quasi-brittle materials. International journal of solid and structures, vol. 46, páginas 3222-3234, 2009.

Yip, W. K., Kong, F. K., Chan, K. S. y Lim, M. K. Statistical model of microcraking of concrete under uniaxial compression. Theoretical and applied fracture mechanics, vol. 22(1), páginas 17-27, 1995.

Zech, B. y Wittmann, F. H. Part II: Probabilistic approach to describe the bahaviour of materials. IASMIRT, 1977.

Zhong, H., Lin, G., Li, X. y Li, J. Seismic failure modeling of concrete dams considering heterogeneity of concrete. Soil Dynamics and Earthquake Engineering, vol. 31, páginas 1678-1689, 2011.

Zhou, X., Dong, W. y Oladiran, O. Assessment of restrained shrinkage cracking of concrete using elliptical ring specimens: experimental and numerical. ASCE Material Civil Engineering, vol. 26(12), páginas 871-878, 2014.

ZHU, H., LI, H. y ZHU, X. On concrete eccentric ring and squared eccentric ring shrinkage test method. Construction and Building Materials, vol. 84, páginas 239-244, 2015.

Zhu, W., Teng, J. G. y TAng, C. A. Mesomechanical model for concrete. Part I: Model development. Magazine of concrete research, vol. 56(6), páginas 313-330, 2004. 

